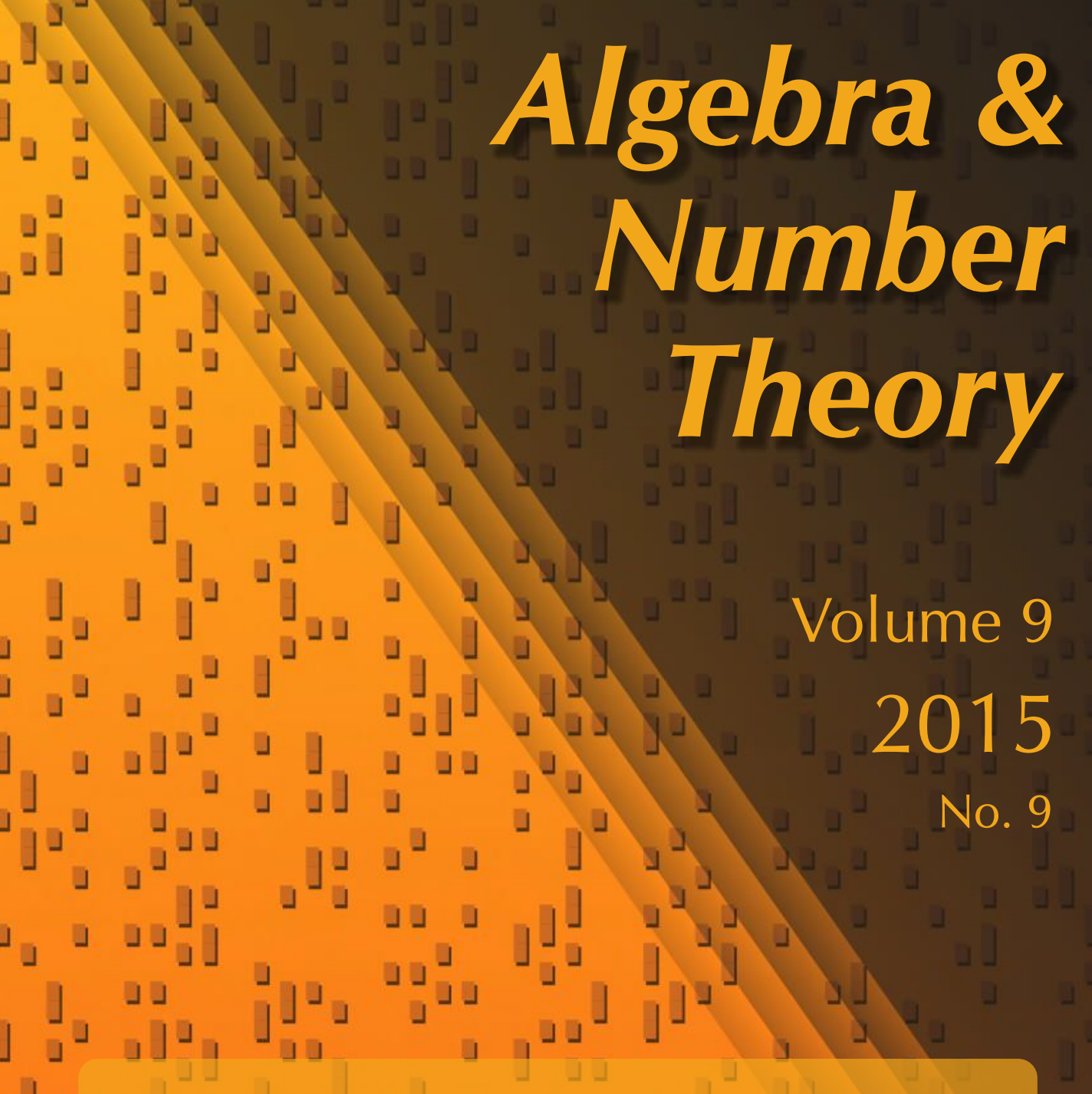

Families of nearly ordinary Eisenstein series on unitary groups

Xin Wan

With an appendix by Kai-Wen Lan

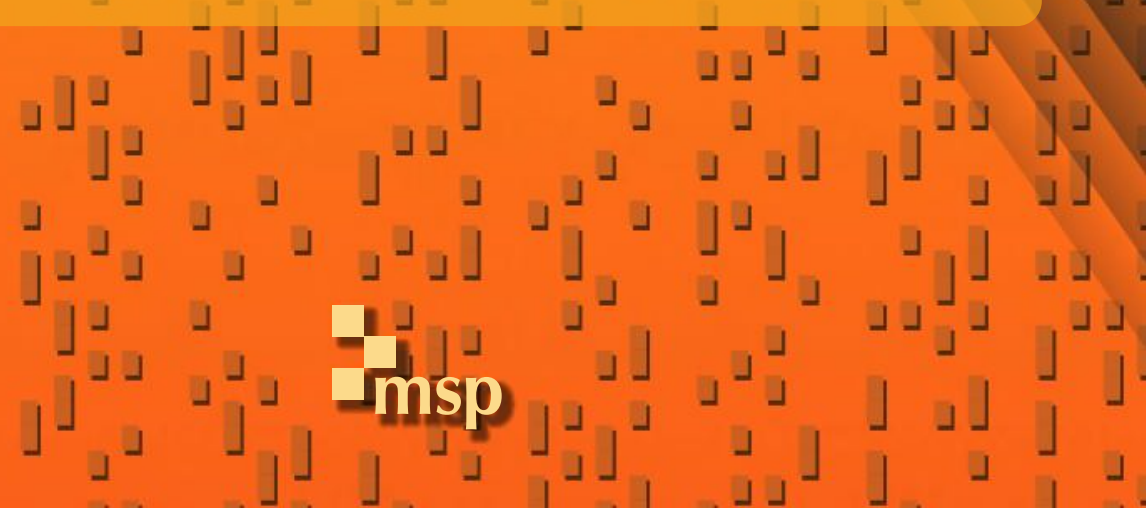




\title{
Families of nearly ordinary Eisenstein series on unitary groups
}

Xin Wan

\author{
With an appendix by Kai-Wen Lan
}

\begin{abstract}
We use the doubling method to construct $p$-adic $L$-functions and families of nearly ordinary Klingen Eisenstein series from nearly ordinary cusp forms on unitary groups of signature $(r, s)$ and Hecke characters, and prove the constant terms of these Eisenstein series are divisible by the $p$-adic $L$-function, following earlier constructions of Eischen, Harris, Li, Skinner and Urban. We also make preliminary computations for the Fourier-Jacobi coefficients of the Eisenstein series. This provides a framework to do Iwasawa theory for cusp forms on unitary groups.
\end{abstract}

1. Introduction

2. Background 1960

3. Eisenstein series and Fourier-Jacobi coefficients 1974

4. Local computations 1984

5. Global computations 2021

Appendix: Boundary strata of connected components in positive characteristics, by Kai-Wen Lan 2035

Acknowledgements 2051

References 2052

A list of symbols can be found on page 2034.

\section{Introduction}

Let $p$ be an odd prime. Let $\mathscr{K}$ be a CM field with the maximal totally real subfield $F$ such that $[F: \mathbb{Q}]=d$. Suppose $p$ is totally split at $\mathscr{K}$. We fix an isomorphism $\iota_{p}:=\mathbb{C}_{p} \simeq \mathbb{C}$ and a $C M$ type $\Sigma_{\infty}$, which means a set of $d$ different embeddings $\mathscr{K} \rightarrow \mathbb{C}$ such that $\Sigma_{\infty} \cup \Sigma_{\infty}^{c}$, where $c$ means complex conjugation, is the set of all embeddings of $\mathscr{K}$ into $\mathbb{C}$. This determines a set of embeddings $\mathscr{K} \hookrightarrow \mathbb{C}_{p}$ using $\iota_{p}$,

Keywords: Iwasawa theory, ordinary, Klingen Eisenstein series, unitary groups, $p$-adic $L$-function, 
which we denote by $\Sigma_{p}$. Let $r \geq s \geq 0$ be integers. We often write $a=r-s$ and $b=s$. Let $\mathrm{U}(r, s)$ be the unitary group associated to the skew-Hermitian matrix

$$
\left(\begin{array}{cc}
{ } & 1^{1_{b}} \\
-1_{b} &
\end{array}\right.
$$

where $\zeta$ is a diagonal matrix such that $i^{-1} \zeta$ is positive definite.

Eischen et al. [ $\geq 2015]$ constructed the $p$-adic $L$-function for an irreducible cuspidal automorphic representation of $\mathrm{U}(r, s)$ that is nearly ordinary at all primes dividing $p$, which interpolates (the algebraic part of) critical values of the standard $L$-function of the representation twisted by general CM characters at far-fromcenter critical points. The main tool used in [loc. cit.] is the doubling method of Piatetski-Shapiro and Rallis. This paper can be thought of as a continuation of their work, but instead using a more general pullback formula of Shimura (which is actually due to Garrett $[1984 ; 1989]$ and is called the "Garrett map") to construct $p$-adic families of Klingen Eisenstein series on $\mathrm{U}(r+1, s+1)$ from the original automorphic representation.

The motivation for doing this is to provide a framework to generalize the important work of Skinner and Urban [2014] on the Iwasawa main conjectures for $\mathrm{GL}_{2}$ to forms on general unitary groups. The general strategy is, starting with a family of cuspforms on the unitary group $\mathrm{U}(r, s)$ and a family of $\mathrm{CM}$ characters, we construct a family of Klingen Eisenstein series on the bigger group $\mathrm{U}(r+1, s+1)$. One tries to prove the constant terms of the Klingen Eisenstein family are divisible by the standard $p$-adic $L$-function of the cuspforms on $\mathrm{U}(r, s)$ and, therefore, the Eisenstein family is congruent to cuspidal families modulo this $p$-adic $L$-function. Passing to the Galois side, such congruences enable us to construct elements in the Selmer groups, proving one divisibility of the corresponding Iwasawa main conjecture.

We have been able to use it to prove one divisibility of the Iwasawa main conjectures for Hilbert modular forms and some kinds of Rankin-Selberg $p$-adic $L$ functions; see [Wan 2013; 2015]. C. Skinner has recently been able to use the result of [Wan 2015] to prove a converse of a theorem of Gross, Zagier and Kolyvagin that states that, if the rank of the Selmer group of an elliptic curve is one and the $p$-part of the Shafarevich-Tate group is finite, then the Heegner point is nontorsion and the central $L$-value vanishes at order exactly one [Skinner 2014]. The first step towards the plan outlined above is to construct the family of Klingen-Eisenstein series and study the $p$-adic properties of its Fourier-Jacobi coefficients, which is the main task of the present paper.

In [Eischen et al. $\geq 2015$ ] the interpolation formulas are proved at all arithmetic points. However, in this paper we are only able to understand the pullback Eisenstein 
sections in the "generic case" (to be defined in Definition 4.42; basically this puts restrictions on the ramification of the form at primes dividing $p$ ). The reason is that it seems difficult in general to describe the nearly ordinary Klingen Eisenstein sections. Fortunately, since along a Hida family the set of forms that are "generic" is Zariski-dense, these computations are enough to construct the whole Hida family of Klingen Eisenstein series (similar to the [Skinner and Urban 2014] case). Thus, we only work with a Hida family of forms instead of a single cusp form, due to this "generic" condition. We remark that when $s=0$, by working with forms of general vector-valued weights, we are able to construct a class of the $p$-adic $L$-function and Klingen Eisenstein family for a single form unramified at $p$ (not necessarily ordinary; see [Eischen and Wan 2014]).

Now we state the main results. Let $\mathscr{K}_{\infty}$ be the maximal abelian pro- $p$-extension of $\mathscr{K}$ unramified outside $p$. We write $\Gamma_{\mathscr{K}}=\operatorname{Gal}\left(\mathscr{K}_{\infty} / \mathscr{K}\right)$. This is a free $\mathbb{Z}_{p}$-module whose rank should be $d+1$, assuming the Leopoldt conjecture. Take a finite extension $L$ over $\mathbb{Q}_{p}$. Let $O_{L}$ be the integer ring of $L$. Let $O_{L}^{\text {ur }}$ be the completion of the integer ring of the maximal unramified extension of $L$. We define $\Lambda_{\mathscr{K}}=0_{L} \llbracket \Gamma_{\mathscr{K}} \rrbracket$. Let $\kappa>4$ be an integer and $\tau_{0}$ a Hecke character of $\mathscr{K}^{\times} \backslash \mathbb{A}_{\mathscr{K}}^{\times}$whose infinite types are $\left(-\frac{1}{2} \kappa, \frac{1}{2} \kappa\right)$ at all infinite places. We have a $\Lambda_{\mathscr{K}}$-valued family of Hecke characters of $\mathscr{K}^{\times} \backslash \mathbb{A}_{\mathscr{K}}^{\times}$containing $\tau_{0}$ as a specialization (to be made precise later). Let $\Lambda$ be the weight algebra for $\mathrm{U}(r, s)$, defined later, and $\square$ a normal domain containing $\Lambda$ which is finite over $\Lambda$. Let $\rrbracket^{\text {ur }}$ be the normalization of an irreducible component of $\square \hat{\otimes}_{O_{L}} \mathcal{O}_{L}^{\text {ur }}$. (In fact, for each such irreducible component we can make the following construction.) Let $\Omega_{\infty} \in \mathbb{C}^{\Sigma_{\infty}}$ be the CM period of the CM field $\mathscr{K}$ and $\Omega_{p} \in\left(\mathbb{Z}_{p}^{\text {ur }}\right)^{\Sigma_{\infty}}$ be the $p$-adic period (we refer to [Hida 2004a] for the definition). We write $\Omega_{\infty}^{\Sigma_{\infty}}$ for the product of the $d$ elements of $\Omega_{\infty}$ and define $\Omega_{p}^{\Sigma_{\infty}}$ similarly. Throughout this paper, we write

$$
\begin{aligned}
z_{\kappa} & =\frac{1}{2}(\kappa-r-s-1), \\
z_{\kappa}^{\prime} & =\frac{1}{2}(\kappa-r-s) .
\end{aligned}
$$

Theorem 1.1. Let $\boldsymbol{f}$ be an \-coefficient, nearly ordinary, cuspidal eigenform on $\mathrm{GU}(r, s)$ such that the specialization $\boldsymbol{f}_{\phi}$ at a Zariski-dense set of "generic" arithmetic points $\phi$ is classical and generates an irreducible automorphic representation of $\mathrm{U}(r, s)$. Let $\Sigma$ be a finite set of primes containing all primes dividing any entry of $\zeta$, or the conductor of $\boldsymbol{f}$, or $\mathscr{H}$.

In the case when $s \neq 0$, we make the assumptions TEMPERED, $\operatorname{Proj}_{f}$ and DUAL, or assumptions TEMPERED, $\operatorname{Proj}_{f}$ and $\operatorname{Proj}_{f^{\vee}}$ (to be defined in Section 5A).

Then:

(i) There is an element $\mathscr{L}_{\boldsymbol{f}, \tau_{0}}^{\Sigma} \in \mathbb{\square}^{\mathrm{ur}} \llbracket \Gamma_{\mathscr{K}} \rrbracket \otimes_{\llbracket \text { ur }} F_{\text {uur }}$ such that, for a Zariski-dense subset of arithmetic points $\phi \in \mathrm{Spec} \square^{\mathrm{ur}} \llbracket \Gamma_{\mathscr{K}} \rrbracket$ (to be specified in Definition 4.42), we have 
that, if $s=0$, then $\mathscr{L}_{\boldsymbol{f}, \tau_{0}}^{\Sigma} \in \square^{\mathrm{ur}} \llbracket \Gamma_{\mathscr{K}} \rrbracket$ and

$$
\begin{aligned}
\phi\left(\mathscr{L}_{\boldsymbol{f}, \tau_{0}}^{\Sigma}\right)=c_{\kappa}^{\prime}\left(z_{\kappa_{\phi}}^{\prime}\right)\left(\frac{(-2)^{-d(a+2 b)}(2 \pi i)^{d(a+2 b) \kappa_{\phi}}(2 / \pi)^{d(a+2 b)(a+2 b-1) / 2}}{\prod_{j=0}^{a+2 b-1}\left(\kappa_{\phi}-j-1\right)^{d}}\right)^{-1} \cdot C_{\boldsymbol{f}_{\phi}}^{p} \\
\times \prod_{v \mid p}\left(\left|p^{t_{1}+\cdots+t_{r}}\right|^{-\kappa_{\phi} / 2} \times p^{-((r+1) / 2) \sum_{j=1}^{r} t_{j}} \prod_{j=1}^{r} \mathfrak{g}\left(\chi_{j} \tau_{1}^{-1}\right) \chi_{j}^{-1} \tau_{1}\left(p^{t_{j}}\right)\right) \\
\times \frac{L^{\Sigma}\left(\tilde{\pi}_{f_{\phi}}, \bar{\tau}_{\phi}^{c}, \kappa_{\phi}-r\right) \Omega_{p}^{r \kappa_{\phi} \Sigma_{\infty}}}{\Omega_{\infty}^{r k_{\phi} \Sigma_{\infty}}} .
\end{aligned}
$$

If $s \neq 0$, then

$\phi\left(\mathscr{L}_{f, \tau_{0}}^{\Sigma}\right)$

$$
\begin{aligned}
=c_{\kappa}^{\prime}\left(z_{\kappa_{\phi}}^{\prime}\right)\left(\frac{(-2)^{-d(a+2 b)}(2 \pi i)^{d(a+2 b) \kappa_{\phi}}(2 / \pi)^{d(a+2 b)(a+2 b-1) / 2}}{\prod_{j=0}^{a+2 b-1}\left(\kappa_{\phi}-j-1\right)^{d}}\right)^{-1} \cdot C_{f_{\phi}}^{p} \\
\times \prod_{v \mid p}\left(\frac{p^{(r+s)(r+s-1) / 2} \cdot(p-1)^{r+s}}{\left(\prod_{i=1}^{r} p^{t_{i} \cdot(r+s-i)}\right) \cdot\left(\prod_{i=1}^{s} p^{t_{r+i}(r+s-i)}\right) \cdot \prod_{j=1}^{r+s}\left(p^{j}-1\right)}\right. \\
\times p^{-s s_{2}(1+a+2 b) / 2} p^{-\sum_{j=1}^{r} t_{j}(a+1) / 2} p^{\sum_{i=1}^{s} t_{r+i}(a+b)}\left|p^{t_{1}+\cdots+t_{r}+s \cdot s_{2}}\right|^{-\kappa_{\phi} / 2} \\
\left.\times \prod_{i=r+1}^{r+s} \mathfrak{g}\left(\chi_{i}^{-1} \tau_{2}\right) \chi_{i} \tau_{2}^{-1}\left(p^{s_{2}}\right) \prod_{j=1}^{r} \mathfrak{g}\left(\chi_{j} \tau_{1}^{-1}\right) \chi_{j}^{-1} \tau_{1}\left(p^{t_{j}}\right)\right) \\
\times \frac{L^{\Sigma}\left(\tilde{\pi}_{f_{\phi}}, \bar{\tau}_{\phi}^{c}, \kappa_{\phi}-r-s\right)}{\left\langle\tilde{\varphi}_{\phi}^{\text {ord }}, \varphi_{\phi}\right\rangle},
\end{aligned}
$$

where the $\chi_{i}$ are defined in Definition 4.42 and $\tau_{\phi, p}=\left(\tau_{1}, \tau_{2}^{-1}\right)$ such that $\tau_{i}$ has conductor $p^{s_{i}}$ with $s_{2}>s_{1}$. Also,

$$
C_{\boldsymbol{f}_{\phi}}^{p}=\prod_{v \nmid p, v \in \Sigma} \tau\left(y_{v} \bar{y}_{v} x_{v}\right)\left|\left(y_{v} \bar{y}_{v}\right)^{2} x_{v} \bar{x}_{v}\right|_{v}^{-z_{\kappa_{\phi}^{\prime}}-(a+2 b) / 2} \operatorname{Vol}\left(\mathfrak{Y}_{v}\right)
$$

(the $x_{v}$ and $y_{v}$ are the $x$ and $y$ in Section $4 C 1$ and $\mathfrak{Y}_{v}$ is defined in Definition 4.11.) The $c_{\kappa}(z)$ and $c_{\kappa}^{\prime}(z)$ are defined in Lemma 4.3 and $\kappa_{\phi}$ is the weight associated to the arithmetic point $\phi$. The $\varphi_{\phi}$ and $\tilde{\varphi}_{\phi}^{\text {ord }}$ are the specialization of $\boldsymbol{f}$ and the $\boldsymbol{f}^{\vee}$ provided by the assumption $\operatorname{Proj}_{f^{\vee}}$ (notice that they are ordinary vectors with respect to different Borel groups, e.g., when $s=0$, the level group for $\varphi_{\phi}$ at $p$ is with respect to the upper-triangular Borel subgroup, while that for $\tilde{\varphi}_{\phi}^{\text {ord }}$ is with respect to the lower-triangular Borel subgroup). The factor

$$
\frac{p^{(r+s)(r+s-1) / 2} \cdot(p-1)^{r+s}}{\left(\prod_{i=1}^{r} p^{t_{i} \cdot(r+s-i)}\right) \cdot\left(\prod_{i=1}^{s} p^{t_{r+i}(r+s-i)}\right) \cdot \prod_{j=1}^{r+s}\left(p^{j}-1\right)}
$$


is the volume of a set $\tilde{K}^{\prime}$ defined in Definition 4.34 (this is smaller than the level group for $\left.\tilde{\varphi}_{\phi}^{\text {ord }}\right)$. The $F_{\square \text { ur }}$ is the fraction field of $\square^{\text {ur }}$. The $\tau_{\phi}$ are specializations of the family of CM characters containing $\tau_{0}$. The $p^{t_{i}}$ are conductors of some characters defined in Definition 4.21. The $\tilde{\lambda}_{\beta, v}$ is defined in (17), whose p-order is $\sum_{i=1}^{b} t_{a+b+i}(a+b-\kappa)$.

(ii) There is a set of formal q-expansions $\boldsymbol{E}_{\boldsymbol{f}, \tau_{0}}:=\left\{\sum_{\beta} a_{[g]}^{h}(\beta) q^{\beta}\right\}_{([g], h)}$ for $\sum_{\beta} a_{[g]}^{h}(\beta) q^{\beta} \in\left(\mathbb{\square}^{\mathrm{ur}} \llbracket \Gamma_{\mathscr{K}} \rrbracket \hat{\otimes}_{\mathbb{Z}_{p}} \mathscr{R}_{[g], \infty}\right) \otimes_{\text {qur }} F_{\text {[ur }}$, where $\mathscr{R}_{[g], \infty}$ is some ring to be defined later, in Section 5, and $([g], h)$ are p-adic cusp labels (Definition 2.6) such that, for a Zariski-dense set of arithmetic points $\phi \in \operatorname{Spec} \square \llbracket \Gamma_{\mathscr{K}} \rrbracket, \phi\left(\boldsymbol{E}_{\boldsymbol{f}, \tau_{0}}\right)$ is the Fourier-Jacobi expansion of the holomorphic, nearly ordinary Klingen Eisenstein series $E\left(f_{\mathrm{Kling}, \phi}, z_{\kappa_{\phi}},-\right)$ we construct in Section $5 C$ (see the interpolation formula in Proposition 5.8). Here, $f_{\text {Kling }}$ is a certain "Klingen section" to be defined there. (iii) The terms $a_{[g]}^{t}(0)$ are divisible by $\mathscr{L}_{\boldsymbol{f}, \tau_{0}}^{\Sigma} \cdot \mathscr{L}_{\bar{\tau}_{0}^{\prime}}^{\Sigma}$, where $\mathscr{L}_{\bar{\tau}_{0}^{\prime}}^{\Sigma}$ is the p-adic L-function of a Dirichlet character to be defined in the text.

The assumption "TEMPERED" is included so that we can easily write down the explicit range of absolute convergence for pullback formulas. It is not serious and may be relaxed using ideas of [Harris 1984]. Besides the theorem, we also make some preliminary computations for the Fourier-Jacobi coefficients for Siegel Eisenstein series. This is crucial for analyzing the $p$-adic properties of the Klingen Eisenstein series we construct. When doing arithmetic application we need to prove that certain Fourier-Jacobi coefficient of this Eisenstein family is prime to the $p$-adic $L$-function.

This paper is organized as follows. In Section 2 we recall various backgrounds. In Section 3 we recall the notion of $p$-adic automorphic forms on unitary groups and Fourier-Jacobi expansion. In Section 4 we recall the notion of Klingen and Siegel Eisenstein series, the pullback formulas relating them and their FourierJacobi coefficients, and then do the local calculations. (This is the most technical part of this paper.) We manage to take the Siegel sections so that, when we are moving our Eisenstein datum $p$-adically, these Siegel Eisenstein series also move $p$-adic analytically. The hard part is to choose the sections at $p$-adic places. At non-Archimedean cases prime to $p$ the choice is more flexible. (We might change this choice whenever doing arithmetic applications; see [Wan 2013; 2015].) At the Archimedean places we restrict ourselves to the parallel scalar weight case, which is enough for doing Hida theory. In Section 5 we make the global calculations and construct the nearly ordinary Klingen Eisenstein series by the pullbacks of a Siegel Eisenstein series from a larger unitary group. Finally, we include an Appendix by Kai-Wen Lan for detailed proofs of some facts used for the $p$-adic $q$-expansion principle. (This is not strictly needed in our construction. But we think it is good to include it for completeness and for the convenience of readers.) 


\section{Background}

In this section we recall notations for holomorphic automorphic forms on unitary groups, Eisenstein series and Fourier-Jacobi expansions.

2A. Notation. Suppose $F$ is a totally real field such that $[F: \mathbb{Q}]=d$ and $\mathscr{K}$ is a totally imaginary quadratic extension of $F$. For a finite place $v$ of $F$ or $\mathscr{K}$, we usually write $\varpi_{v}$ for a uniformizer and $q_{v}$ for the cardinality of its residue field. Let $c$ be the nontrivial element of $\operatorname{Gal}(\mathscr{K} / F)$. Let $r$ and $s$ be two integers with $r \geq s \geq 0$. We fix an odd prime $p$ that splits completely in $\mathscr{K} / \mathbb{Q}$. We fix $i_{\infty}: \overline{\mathbb{Q}} \hookrightarrow \mathbb{C}$ and $\iota: \mathbb{C} \simeq \mathbb{C}_{p}$ and write $i_{p}$ for $\iota \circ i_{\infty}$. Let $\Sigma_{\infty}$ be the set of Archimedean places of $F$. We take a CM type of $\mathscr{K}$, still denoted by $\Sigma_{\infty}$ (thus $\Sigma_{\infty} \sqcup \Sigma_{\infty}^{c}$ are all embeddings $\mathscr{K} \rightarrow \mathbb{C}$, where $\left.\Sigma_{\infty}^{c}=\left\{\tau \circ c \mid \tau \in \Sigma_{\infty}\right\}\right)$.

We use $\epsilon$ to denote the cyclotomic character and $\omega$ the Teichmüller character. We will often adopt the following notation: for an idele class character $\chi=\bigotimes_{v} \chi_{v}$, we write $\chi_{p}(x)=\prod_{v \mid p} \chi_{v}\left(x_{v}\right)$. For a character $\psi$ of $\mathscr{K}_{v}^{\times}$or $\mathbb{A}_{\mathscr{K}}^{\times}$, we often write $\psi^{\prime}$ for the restriction to $F_{v}^{\times}$or $\mathbb{A}_{F}^{\times}$. For a character $\tau$ of $\mathscr{K}^{\times}$or $\mathbb{A}_{\mathscr{K}}^{\times}$, we define $\tau^{c}$ by $\tau^{c}(x)=\tau\left(x^{c}\right)$. (Note: we will write $\bar{\tau}(x)$ for the complex conjugation of $\tau(x)$ while the " $c$ " means taking complex conjugation for the source.)

If $v$ is a prime of $F$ with characteristic $\ell$ and $\mathfrak{d}_{v} \mathscr{O}_{F, v}=\left(d_{v}\right), d_{v} \in F_{v}^{\times}$is the different of $F / \mathbb{Q}$ at $v$ and, if $\psi_{v}$ is a character of $F_{v}^{\times}$and $\left(c_{\psi, v}\right) \subset \mathbb{O}_{F, v}$ is the conductor, then we define the local Gauss sums

$$
\mathfrak{g}\left(\psi_{v}, c_{\psi, v} d_{v}\right):=\sum_{a \in\left(\mathbb{O}_{F, v} / c_{\psi, v}\right)^{\times}} \psi_{v}(a) e\left(\operatorname{Tr}_{F_{v} / \mathbb{Q}_{\ell}}\left(\frac{a}{c_{\psi, v} d_{v}}\right)\right),
$$

where $\ell$ is the rational prime above $v$. If $\otimes \psi_{v}$ is an idele class character of $\mathbb{A}_{F}^{\times}$ then we set the global Gauss sum,

$$
\mathfrak{g}\left(\otimes \psi_{v}\right):=\prod_{v} \psi_{v}^{-1}\left(c_{\psi, v} d_{v}\right) \mathfrak{g}\left(\psi, c_{\psi, v} d_{v}\right)
$$

This is independent of all the choices of $d_{v}$ and $C_{\psi, v}$. Also, if $F_{v} \simeq \mathbb{Q}_{p}$ and $\left(p^{t}\right)$ is the conductor for $\psi_{v}$, then we write $\mathfrak{g}\left(\psi_{v}\right):=\mathfrak{g}\left(\psi_{v}, p^{t}\right)$. We define the Gauss sums for $\mathscr{K}$ similarly.

Let $\mathscr{K}_{\infty}$ be the maximal abelian $\mathbb{Z}_{p}$-extension of $\mathscr{K}$ unramified outside $p$. Write $\Gamma_{\mathscr{K}}:=\operatorname{Gal}\left(\mathscr{K}_{\infty} / \mathscr{K}\right)$ and $G_{\mathscr{K}}$ the absolute Galois group of $\mathscr{K}$. Define $\Lambda_{\mathscr{K}}:=\mathbb{Z}_{p} \llbracket \Gamma_{\mathscr{K}} \rrbracket$. For any finite extension $A$ of $\mathbb{Z}_{p}$ define $\Lambda_{\mathscr{K}, A}:=A \llbracket \Gamma_{\mathscr{K}} \rrbracket$. Let $\varepsilon_{\mathscr{K}}: G_{\mathscr{K}} \rightarrow \Gamma_{\mathscr{K}} \hookrightarrow \Lambda_{\mathscr{K}}^{\times}$ be the canonical character. We define $\Psi_{\mathscr{K}}$ to be the composition of $\varepsilon_{\mathscr{K}}$ with the reciprocity map of global class field theory, which we denote as $\operatorname{rec}_{\mathscr{K}}: \mathscr{K}^{\times} \backslash \mathbb{A}_{\mathscr{K}}^{\times} \rightarrow G_{\mathscr{K}}^{\mathrm{ab}}$. Here we used the geometric normalization of class field theory. We make the corresponding definitions for $F$ as well. 
Let $S_{m}(R)$ be the set of matrices $S \in M_{m}\left(R \otimes_{\mathscr{O}_{F}} \mathscr{O}_{\mathscr{K}}\right)$ such that $S={ }^{t} \bar{S}$, where conjugation is with respect to the second variable of $R \otimes_{\mathscr{O}_{F}} \mathcal{O}_{\mathscr{K}}$. We write $B=B_{n}$ and $N=N_{n}$ for the upper-triangular Borel subgroup and unipotent radical of the group $\mathrm{GL}_{n}$. Let $N^{\text {opp }}$ or $N^{-}$be the opposite unipotent radical of $N$. We define the function $e_{\mathbb{A}_{\mathbb{Q}}}=\prod_{v} e_{v}$ with $e_{v}$ the function on $\mathbb{Q}_{v}^{\times}$such that $e_{v}\left(x_{v}\right)=e^{2 \pi i \cdot\left\{x_{v}\right\}}$ for $\left\{x_{v}\right\}$ the fractional part of $x_{v}$ and $e_{\infty}(x)=e^{-2 \pi i x}$. We will usually write $\eta=\left({ }_{-1_{m}}{ }^{1}{ }_{m}\right)$ if $m$ is clear from the context.

2B. Unitary Groups. We define

$$
\theta_{r, s}=\left(\begin{array}{cc}
{ }^{1_{s}} \\
-1_{s}
\end{array}\right.
$$

where $\zeta$ is a fixed diagonal matrix such that $i^{-1} \zeta$ is totally positive. Let $V=V(r, s)$ be the skew-Hermitian space over $\mathscr{K}$ with respect to this metric, i.e., $\mathscr{K}^{r+s}$ equipped with the metric given by $\langle u, v\rangle:=u \theta_{r, s}{ }^{t} \bar{v}$. We define algebraic groups $\operatorname{GU}(r, s)$ and $\mathrm{U}(r, s)$ as follows: for any $\mathscr{O}_{F}$-algebra $R$, the $R$ points are

$$
\mathrm{GU}(r, s)(R):=\left\{g \in \mathrm{GL}_{r+s}\left(\mathscr{O}_{\mathscr{K}} \otimes_{\mathscr{O}_{F}} R\right) \mid g \theta_{r, s} g^{*}=\mu(g) \theta_{r, s}, \mu(g) \in R^{\times}\right\}
$$

(where $g^{*}={ }^{t} \bar{g}$ and $\mu: \mathrm{GU}(r, s) \rightarrow \mathbb{G}_{m, F}$ is called the similitude character) and

$$
\mathrm{U}(r, s)(R):=\{g \in \mathrm{GU}(r, s)(R) \mid \mu(g)=1\} .
$$

So the unitary group $\mathrm{U}(r, s)$ in this paper really means the unitary group with respect to our fixed metric $\theta_{r, s}$. Sometimes we write $\mathrm{GU}_{n}$ and $\mathrm{U}_{n}$ for $\mathrm{GU}(n, n)$ and $\mathrm{U}(n, n)$. For two forms $\varphi_{1}, \varphi_{2}$ on $\mathrm{U}(r, s)\left(\mathbb{A}_{F}\right)$, we define the inner product by

$$
\left\langle\varphi_{1}, \varphi_{2}\right\rangle:=\int_{\mathrm{U}(r, s)(F) \backslash \mathrm{U}(r, s)\left(\mathrm{A}_{F}\right)} \varphi_{1}(g) \varphi_{2}(g) d g,
$$

where the measure is chosen so that $\mathrm{U}(r, s)\left(0_{F_{v}}\right)=1$ for all finite $v$ and we take the measure at Archimedean places as in [Shimura 1997, (7.14.5)].

We have the embedding

$$
\begin{aligned}
& \mathrm{GU}(r, s) \times \operatorname{Res}_{\mathscr{O Y K}_{K} / \mathscr{O}_{F}} \mathbb{G}_{m} \rightarrow \mathrm{GU}(r+1, s+1), \\
& g \times x=\left(\begin{array}{lll}
a & b & c \\
d & e & f \\
h & l & k
\end{array}\right) \times x \mapsto\left(\begin{array}{lllll}
a & & b & c & \\
& \mu(g) \bar{x}^{-1} & & & \\
d & & e & f & \\
h & & l & k & \\
& & & & x
\end{array}\right) .
\end{aligned}
$$

We write $m(g, x)$ for the right-hand side. The image of the above map is the Levi subgroup of the Klingen parabolic subgroup $P$ of $\mathrm{GU}(r+1, s+1)$, which 
consists of matrices in $\mathrm{GU}(r+1, s+1)$ such that the off-diagonal entries of the $(s+1)$-st column and the last row are 0 . We denote this Levi subgroup by $M_{P}$. We also write $N_{P}$ for the unipotent radical of $P$. We also define $B=B(r, s)$ to be the standard Borel subgroup, consisting of matrices

$$
g=\left(\begin{array}{cc}
A_{g} & B_{g} \\
& D_{g}
\end{array}\right),
$$

where the blocks are with respect to the partition $r+s$ and we require that $A_{g}$ is lower-triangular and $D_{g}$ is upper-triangular.

We write $-V(r, s)=V(s, r)$ for the Hermitian space whose metric is $-\theta_{r, s}$. We define some embeddings of $\mathrm{GU}(r+1, s+1) \times \mathrm{GU}(-V(r, s))$ into some larger unitary groups. These will be used in the doubling method. Recall we wrote $a=r-s$ and $b=s$ at the beginning of the introduction; we define $\mathrm{GU}(r+s+1, r+s+1)^{\prime}$ to be the unitary similitude group associated to

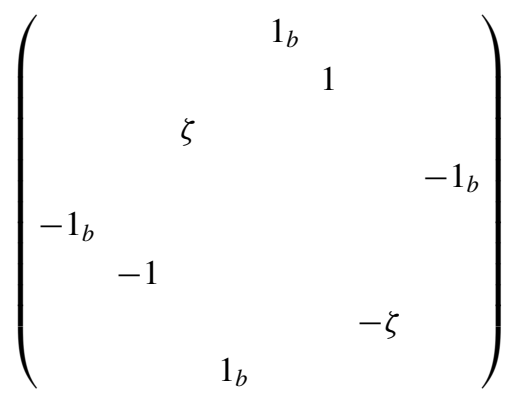

and $G(r+s, r+s)^{\prime}$ to be associated to

$$
\left(\begin{array}{cccc} 
& & 1_{b} & \\
& & & \\
-1_{b} & & & \\
& & & -\zeta
\end{array}\right)
$$

We define an embedding

$$
\begin{aligned}
& \alpha:\left\{g_{1} \times g_{2} \in \mathrm{GU}(r+1, s+1) \times \mathrm{GU}(-V(r, s)) \mid \mu\left(g_{1}\right)=\mu\left(g_{2}\right)\right\} \\
& \rightarrow \mathrm{GU}(r+s+1, r+s+1)^{\prime}
\end{aligned}
$$

by viewing $g_{1}$ as a block matrix with respect to the partition $s+1+(r-s)+s+1$ (this means we use this partition to divide both the rows and the columns into blocks) and $g_{2}$ as a block matrix with respect to $s+(r-s)+s$, then we define $\alpha$ by requiring the 1, 2, 3, 4, 5-th (blockwise) rows and columns of $\mathrm{GU}(r+1, s+1)$ embed to the 1, 2, 3, 5, 6-th (blockwise) rows and columns of $\mathrm{GU}(r+s+1, r+s+1)^{\prime}$ and the 
1, 2, 3-rd (blockwise) rows and columns of $\mathrm{GU}(V(s, r))$ embed to the 8, 7, 4-th rows and columns (blockwise) of $\mathrm{GU}(r+s+1, r+s+1)^{\prime}$.

We also define an embedding

$$
\alpha^{\prime}:\left\{g_{1} \times g_{2} \in \mathrm{GU}(r, s) \times \mathrm{GU}(-V(r, s)) \mid \mu\left(g_{1}\right)=\mu\left(g_{2}\right)\right\} \rightarrow \mathrm{GU}(r+s, r+s)^{\prime}
$$

in a similar way to above: Consider $\mathrm{GU}(r, s)$ and $\mathrm{GU}(-V(r, s))$ as block matrices with respect to the partition $s+(r-s)+s$. Put the 1,2,3-rd (blockwise) rows and columns of the first $\mathrm{GU}(r, s)$ into the 1,2, 4-th (blockwise) rows and columns of $\mathrm{GU}(r+s, r+s)^{\prime}$ and put the 1,2,3-rd (blockwise) rows and columns of the second $\mathrm{GU}(r, s)$ into the 6,5 , 4-th rows and columns of $\mathrm{GU}(r+s, r+s)^{\prime}$.

We also define isomorphisms

$$
\beta: \mathrm{GU}(r+s+1, r+s+1)^{\prime} \stackrel{\sim}{\longrightarrow} \mathrm{GU}(r+s+1, r+s+1), \quad g \mapsto S^{-1} g S,
$$

and

$$
\beta^{\prime}: \mathrm{GU}(r+s, r+s)^{\prime} \stackrel{\sim}{\longrightarrow} \mathrm{GU}(r+s, r+s), \quad g \mapsto S^{\prime-1} g S^{\prime},
$$

where

and

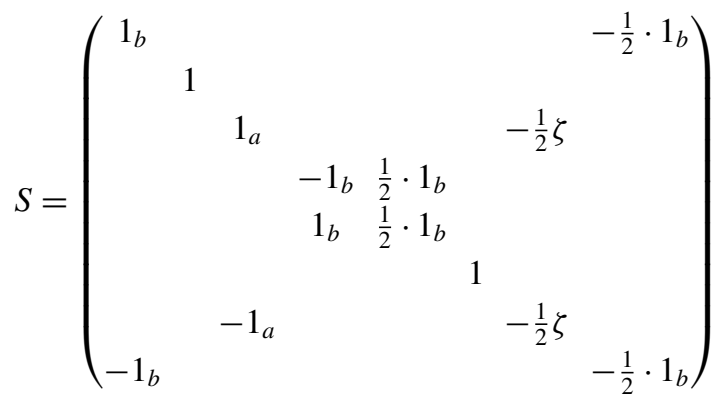

$$
S^{\prime}=\left(\begin{array}{cccccc}
1_{b} & & & & & \\
& 1_{a} & & & -\frac{1}{2} \cdot 1_{b} \\
& & -1_{b} & \frac{1}{2} \cdot 1_{b} & & \\
& & 1_{b} & \frac{1}{2} \cdot 1_{b} & & \\
& -1_{a} & & & -\frac{1}{2} \zeta & \\
-1_{b} & & & & & -\frac{1}{2} \cdot 1_{b}
\end{array}\right) .
$$

Remark 2.1 (about unitary groups). In order to have Shimura varieties for doing $p$-adic modular forms and Galois representations, we need to use a unitary group defined over $\mathbb{Q}$. More precisely, consider $V$ as a skew-Hermitian space over $\mathbb{Q}$ and still write $\theta_{r, s}$ for the metric on it. Let $T$ be a $O_{\mathscr{K}}$ lattice that we use to define $\mathrm{GU}(r, s)$. Then the correct unitary similitude group should be

$$
\mathrm{GU}^{0}(r, s)(A):=\left\{g \in \mathrm{GL}_{\bigcirc_{r c} \otimes_{\mathbb{Z}} A}\left(T \otimes_{\mathbb{Z}} A\right) \mid g \theta_{r, s} g^{*}=\mu(g) \theta_{r, s}, \mu(g) \in A\right\}
$$

for any commutative ring $A$. This group is smaller than the one we defined before. However, this group is not convenient for local computations, since we cannot treat 
the primes of $F$ independently. So what we do (implicitly) is: For the analytic construction, we write down forms on the larger unitary similitude group defined above and then restrict to the smaller one. For the algebraic construction, we only do the pullbacks for unitary (instead of similitude) groups.

We are going to fix some bases of the various Hermitian spaces. We let

$$
y^{1}, \ldots, y^{s}, w^{1}, \ldots, w^{r-s}, x^{1}, \ldots, x^{s}
$$

be the standard basis of $V$ such that the Hermitian forms is given above. Let $W$ be the span over $\mathscr{K}$ of $w^{1}, \ldots, w^{r-s}$. Let $X^{\vee}=\mathscr{O}_{\mathscr{K}} x^{1} \oplus \cdots \oplus \mathscr{O}_{\mathscr{K}} x^{s}$ and $Y=\mathscr{O}_{\mathscr{K}} y^{1} \oplus \cdots \oplus \mathscr{O}_{\mathscr{K}} y^{s}$. Let $L$ be an $\mathscr{O}_{\mathscr{K}}$-maximal lattice such that $L_{p}:=L \otimes_{\mathbb{Z}} \mathbb{Z}_{p}=$ $\sum_{i=1}^{r-s}\left(\mathscr{O}_{\mathscr{K}} \otimes_{\mathbb{Z}} \mathbb{Z}_{p}\right) w^{i}$. We define a $\mathscr{O}_{\mathscr{K}}$-lattice $M$ of $V$ by

$$
M:=Y \oplus L \oplus X^{\vee} .
$$

Let $M_{p}=M \otimes_{\mathbb{Z}} \mathbb{Z}_{p}$. A pair of sublattices $\operatorname{Pol}_{p}=\left\{N^{-1}, N^{0}\right\}$ of $M_{p}$ is called an ordered polarization of $M_{p}$ if $N^{-1}$ and $N^{0}$ are maximal isotropic direct summands in $M_{p}$ and they are dual to each other with respect to the Hermitian pairing. Moreover, we require that, for each $v=w w^{c}$ with $w \in \Sigma_{p}$, rank $N_{w}^{-1}=\operatorname{rank} N_{w^{c}}^{0}=r$ and rank $N_{w^{c}}^{-1}=\operatorname{rank} N_{w}^{0}=s$. The standard polarization of $M_{p}$ is given by $M_{v}^{-1}=Y_{w} \oplus L_{w} \oplus Y_{w^{c}}$ and $M_{v}^{0}=X_{w^{c}} \oplus L_{w^{c}} \oplus X_{w}$. We let $-V$ be the Hermitian space $V$ with the metric given by the negative of $V$. We let $\tilde{y}^{1}, \ldots, \tilde{y}^{s}$, $\tilde{w}^{1}, \ldots, \tilde{w}^{r-s}, \tilde{x}^{1}, \ldots, \tilde{x}^{s}$ be the corresponding basis. Let $\mathscr{K} y^{s+1} \oplus \mathscr{K} x^{s+1}$ be a 2-dimensional Hermitian space with metric $\left({ }_{-1}^{1}\right)$. We define

$$
\boldsymbol{W}:=V \oplus \mathscr{K} y^{s+1} \oplus \mathscr{K} x^{s+1} \oplus(-V) .
$$

Let $\Upsilon \in \mathrm{U}(n+1, n+1)\left(F_{p}\right)$ be such that, for each $v \mid p$ with $v=w w^{c}$, where $w$ is in our $p$-adic CM type $\Sigma_{p}, \Upsilon_{w}=S_{w}^{-1}$. We define another basis of $\boldsymbol{W}$ by

$$
\begin{array}{r}
{ }^{t}\left(y^{1}, \ldots, y^{s+1}, w^{1}, \ldots, w^{r-s}, x^{1}, \ldots, x^{s+1}, y^{1}, \ldots, y^{s}, w^{1}, \ldots, w^{r-s}, x^{1}, \ldots, x^{s}\right) \Upsilon \\
={ }^{t}\left(\boldsymbol{y}^{1}, \ldots, \boldsymbol{y}^{r+s+1}, \boldsymbol{x}^{1}, \ldots, \boldsymbol{x}^{r+s+1}\right) .
\end{array}
$$

Then $\boldsymbol{Y}:=\bigoplus_{i=1}^{r+s+1}\left(\mathscr{O}_{\mathscr{K}} \otimes_{\mathbb{Z}} \mathbb{Z}_{p}\right) \boldsymbol{y}^{i}$ and $\boldsymbol{X}:=\bigoplus_{i=1}^{r+s+1}\left(\mathcal{O}_{\mathscr{K}} \otimes_{\mathbb{Z}} \mathbb{Z}_{p}\right) \boldsymbol{x}^{i}$ gives another polarization $(\boldsymbol{Y}, \boldsymbol{X})$ of $\boldsymbol{L}_{p}:=M_{p} \oplus\left(-M_{p}\right) \oplus \mathcal{O}_{\mathscr{K}} y^{s+1} \oplus \mathcal{O}_{\mathscr{K}} x^{s+1}$.

\section{C. Automorphic forms.}

2C1. Hermitian symmetric domain. Suppose $r \geq s>0$. Then the Hermitian symmetric domain for $G:=\mathrm{GU}(r, s)$ is

$$
X^{+}=X_{r, s}=\left\{\tau=\left(\begin{array}{l}
x \\
y
\end{array}\right) \mid x \in M_{s}\left(\mathbb{C}^{\Sigma}\right), y \in M_{(r-s) \times s}\left(\mathbb{C}^{\Sigma}\right), i\left(x^{*}-x\right)>-i y^{*} \theta^{-1} y\right\} .
$$


For $\alpha \in \mathrm{GU}(r, s)\left(F_{\infty}\right)$, where $F_{\infty}:=F \otimes_{\mathbb{Q}} \mathbb{R}$, we write

$$
\alpha=\left(\begin{array}{lll}
a & b & c \\
d & e & f \\
h & l & d
\end{array}\right)
$$

according to the standard basis of $V$ together with the block decomposition with respect to $s+(r-s)+s$. There is an action of $\alpha \in G\left(F_{\infty}\right)^{+}$(here the superscript + means the component with positive similitude factor at all Archimedean places) on $X_{r, s}$, defined by

$$
\alpha\left(\begin{array}{l}
x \\
y
\end{array}\right)=\left(\begin{array}{l}
a x+b y+c \\
g x+e y+f
\end{array}\right)(h x+l y+d)^{-1} .
$$

If $r s=0, X_{r, s}$ consists of a single point, written $\boldsymbol{x}_{0}$, with the trivial action of $G\left(F_{\infty}\right)^{+}$. For an open compact subgroup $U$ of $G\left(\mathbb{A}_{F, f}\right)$, put

$$
M_{G}\left(X^{+}, U\right):=G(F)^{+} \backslash X^{+} \times G\left(\mathbb{A}_{F, f}\right) / U,
$$

where $U$ is an open compact subgroup of $G\left(\mathbb{A}_{F, f}\right)$. We let

$$
\mathbb{C}^{r, s}=\mathbb{C}\left(\Sigma^{c}\right)^{s} \otimes \mathbb{C}\left(\Sigma^{c}\right)^{r-s} \otimes \mathbb{C}(\Sigma)^{s}
$$

and define a map $c_{r, s}$ on it by $\left(u_{1}, u_{2}, u_{3}\right) c_{r, s}=\left(\bar{u}_{1}, \bar{u}_{2}, u_{3}\right)$. We define the map $p(\tau): V \otimes_{\mathbb{Q}} \mathbb{R} \simeq \mathbb{C}^{r, s}$ by $p(\tau) v=v B(\tau) c_{r, s}$. Let

$$
B(\tau)=\left(\begin{array}{lll}
x^{*} & y^{*} & x \\
0 & \zeta & y \\
1_{s} & 0 & 1_{s}
\end{array}\right) .
$$

We define the automorphic factors $\kappa(\alpha, \tau)$ and $\mu(\alpha, \tau)$ by

$$
\alpha B(\tau)=B(\alpha \tau)(\overline{\kappa(\alpha, \tau)}, \mu(\alpha, \tau))
$$

for $\alpha \in G(\mathbb{R})$ and $\tau \in X^{+}$. We sometimes write $\kappa_{r, s}(\alpha, \tau), \mu_{r, s}(\alpha, \tau)$ to emphasize the group $\mathrm{U}(r, s)$. We define $j(g, z):=\operatorname{det}(\mu(g, z))$. For $z \in X_{r+1, s+1}$, we define $\wp(z) \in X_{r, s}$ to be the lower-right $r \times s$ submatrix. For $z_{1}=\left(\begin{array}{l}x_{1} \\ y_{1}\end{array}\right)$ and $z \in\left(\begin{array}{l}x \\ y\end{array}\right)$, we define $\eta\left(z_{1}, z\right)=i\left(x_{1}^{*}-x\right)-y_{1}^{*}\left(i \zeta^{-1}\right) y$ and $\delta\left(z_{1}, z\right)=2^{-s} \operatorname{det}\left(\eta\left(z_{1}, z\right)\right)$.

2C2. Automorphic forms. We will mainly follow [Hsieh 2014] to define the space of automorphic forms, with slight modifications. We define a cocycle

$$
\begin{aligned}
J: R_{F / \mathbb{Q}} G(\mathbb{R})^{+} \times X^{+} & \rightarrow \mathrm{GL}_{r}\left(\mathbb{C}^{\Sigma}\right) \times \mathrm{GL}_{s}\left(\mathbb{C}^{\Sigma}\right):=H(\mathbb{C}), \\
(\alpha, \tau) & \mapsto(\kappa(\alpha, \tau), \mu(\alpha, \tau)),
\end{aligned}
$$


where $\kappa(\alpha, \tau)=\left(\begin{array}{cc}\bar{h}^{t} x+\bar{d} & \bar{h}^{t} y+l \bar{\theta} \\ -\bar{\theta}^{-1}\left(\bar{g}^{t} x+\bar{f}\right) & -\bar{\theta}^{-1} \bar{g}^{t} y+\bar{\theta}^{-1} \bar{e} \bar{\theta}\end{array}\right) \quad$ and $\quad \mu(\alpha, \tau)=h x+l y+d$

$$
\text { for } \tau=\left(\begin{array}{l}
x \\
y
\end{array}\right) \text { and } \alpha=\left(\begin{array}{lll}
a & b & c \\
g & e & f \\
h & l & d
\end{array}\right) \text {. }
$$

Let $\boldsymbol{i}$ be the point $\left(\begin{array}{c}i 1_{s} \\ 0\end{array}\right)$ on the Hermitian symmetric domain for $\mathrm{GU}(r, s)$ (here 0 means the $(r-s) \times s$ matrix 0$)$. Let $\mathrm{GU}(r, s)(\mathbb{R})^{+}$be the subgroup of $\mathrm{GU}(r, s)(\mathbb{R})$ whose similitude factor is totally positive. Let $K_{\infty}^{+}$be the compact subgroup of $\mathrm{U}(r, s)(\mathbb{R})$ stabilizing $i$ and $K_{\infty}$ be the group generated by $K_{\infty}^{+}$and $\operatorname{diag}\left(1_{r+s},-1_{s}\right)$. Then $J: K_{\infty}^{+} \rightarrow H(\mathbb{C}), k_{\infty} \mapsto J\left(k_{\infty}, i\right)$, defines an algebraic representation of $K_{\infty}^{+}$.

Definition 2.2. A weight $\underline{k}$ is defined by a set $\left\{\underline{k}_{\sigma}\right\}_{\sigma \in \Sigma_{\infty}}$ where

$$
\underline{k}_{\sigma}=\left(c_{r+s, \sigma}, \ldots, c_{s+1, \sigma} ; c_{1, \sigma}, \ldots, c_{s, \sigma}\right)
$$

with $c_{1, \sigma} \geq \cdots \geq c_{s, \sigma} \geq c_{s+1, \sigma}+r+s \geq \cdots \geq c_{s+r, \sigma}+r+s$ for the $c_{i, \sigma}$ in $\mathbb{Z}$.

Remark 2.3. Our convention is different from others in the literature. For example, in [Hsieh 2014] the $a_{r+1-i}$ there for $1 \leq i \leq r$ is our $-c_{s+i}$, and $b_{s+1-j}$ there for $1 \leq j \leq s$ is our $c_{j}$. We let $\underline{k}^{\prime}:=\left(a_{1}, \ldots, a_{r} ; b_{1}, \ldots, b_{s}\right)$. We also note that if each $\underline{k}_{\sigma}=(0, \ldots, 0 ; \kappa, \ldots, \kappa)$ then $L^{\underline{k}}(\mathbb{C})$ is 1 -dimensional with $\rho^{\underline{k}}(h)=\operatorname{det} \mu(h, i)^{\kappa}$.

For a weight $\underline{k}=\left(c_{r+s}, \ldots, c_{s+1} ; c_{1}, \ldots, c_{s}\right)$, we define the representation of $\mathrm{GL}_{r} \times \mathrm{GL}_{s}$ with minimal weight $-\underline{k}$ by

$$
L_{\underline{k}}=\left\{f \in \mathrm{O}_{\mathrm{GL}_{r} \times \mathrm{GL}_{s}} \mid f\left(t n_{+} g\right)=\underline{k}^{\prime-1}(t) f(g), t \in T_{r} \times T_{s}, n_{+} \in N_{r} \times{ }^{t} N_{s}\right\},
$$

where $\mathrm{O}_{\mathrm{GL}} \times \mathrm{GL}_{s}$ is the structure sheaf of the algebraic group $\mathrm{GL}_{r} \times \mathrm{GL}_{s}$; see [Hsieh 2014, Section 3]. The group action is denoted by $\rho_{\underline{k}}$. We define the functional $/ l_{\underline{k}}$ on $L_{k}$ by evaluating at the identity and define a model $L^{\underline{k}}(\mathbb{C})$ of the representation $H(\overline{\mathbb{C}})$ with the highest weight $\underline{k}$ as follows. The underlying space of $L^{\underline{k}}(\mathbb{C})$ is $L_{k}(\mathbb{C})$ and the group action is defined by

$$
\rho^{\underline{k}}(h)=\rho_{\underline{k}}\left({ }^{t} h^{-1}\right), \quad h \in H(\mathbb{C}) .
$$

For a weight $\underline{k}$, define $\|\underline{k}\|=\left\{\|\underline{k}\|_{\sigma}\right\}_{\sigma \in \Sigma} \in \mathbb{Z}^{\Sigma}$ by

$$
\|\underline{k}\|_{\sigma}:=-c_{s+1, \sigma}-\cdots-c_{s+r, \sigma}+c_{1 \sigma}+\cdots+c_{s, \sigma}
$$

and $|\underline{k}| \in \mathbb{Z}^{\Sigma \sqcup \Sigma^{c}}$ by

$$
|\underline{k}|=\sum_{\sigma \in \Sigma}\left(c_{1, \sigma}+\cdots+c_{s, \sigma}\right) \cdot \sigma-\left(c_{s+1, \sigma}+\cdots+c_{s+r, \sigma}\right) \cdot \sigma^{c} .
$$


Let $\chi$ be a Hecke character of $\mathscr{K}$ with infinite type $|\underline{k}|$, i.e., the Archimedean part of $\chi$ is given by

$$
\chi\left(z_{\infty}\right)=\left(\prod_{\sigma} z_{\sigma}^{\left(c_{1, \sigma}+\cdots+c_{s, \sigma}\right)} \cdot z_{\sigma^{c}}^{-\left(c_{s+1, \sigma}+\cdots+c_{s+r, \sigma}\right)}\right) .
$$

Definition 2.4. Let $U$ be an open compact subgroup in $G\left(\mathbb{A}_{F, f}\right)$. We denote by $M_{\underline{k}}(U, \mathbb{C})$ the space of holomorphic $L^{\underline{k}}(\mathbb{C})$-valued functions $f$ on $X^{+} \times G\left(\mathbb{A}_{F, f}\right)$ such that, for $\tau \in X^{+}, \alpha \in G(F)^{+}$and $u \in U$, we have

$$
f(\alpha \tau, \alpha g u)=\mu(\alpha)^{-\|\underline{k}\|} \rho^{\underline{k}}(J(\alpha, \tau)) f(\tau, g) .
$$

Now we consider automorphic forms on unitary groups in the adelic language. Let $\boldsymbol{i} \in X^{+}$and $K_{\infty}^{+} \subset \mathrm{U}(r, s)\left(F_{\infty}\right)$ be the stabilizer of $\boldsymbol{i}$. The space of automorphic forms of weight $\underline{k}$ and level $U$ with central character $\chi$ consists of smooth and slowly increasing functions $F: G\left(\mathbb{A}_{F}\right) \rightarrow L_{k}(\mathbb{C})$ such that, for every $\left(\alpha, k_{\infty}, u, z\right) \in G(F) \times K_{\infty}^{+} \times U \times Z\left(\mathbb{A}_{F}\right)$,

$$
F\left(z \alpha g k_{\infty} u\right)=\rho^{\underline{k}}\left(J\left(k_{\infty}, i\right)^{-1}\right) F(g) \chi^{-1}(z) .
$$

2C3. The group $\mathrm{GU}(s, r)$. Now we consider the unitary group $\mathrm{GU}(s, r)$ which has the same Hermitian space as $\mathrm{GU}(r, s)$ but with the metric $\langle,\rangle_{s, r}:=-\langle,\rangle_{r, s}$. We define the symmetric domain $X_{s, r}=X_{r, s}$ but with the complex structure such that a function is holomorphic on $X_{s, r}$ if and only if it is holomorphic on $X_{r, s}$ after composition with the map

$$
X_{r, s} \rightarrow X_{s, r}, \quad\left(\begin{array}{l}
x \\
y
\end{array}\right) \mapsto\left(\begin{array}{l}
-\bar{x} \\
-\bar{y}
\end{array}\right) .
$$

We let $\mathbb{C}^{s, r}=\mathbb{C}(\Sigma)^{s} \otimes \mathbb{C}(\Sigma)^{r-s} \otimes \mathbb{C}\left(\Sigma^{c}\right)^{s}$ and define $c_{s, r}$ by $\left(u_{1}, u_{2}, u_{3}\right) c_{s, r}=$ $\left(u_{1}, u_{2}, \bar{u}_{3}\right)$. For $\mathrm{GU}(s, r)$, we define $p(\tau): V \otimes_{\mathbb{Q}} \mathbb{R} \simeq \mathbb{C}^{s, r}$ by $p(\tau) v=v B(\tau) c_{s, r}$. We define the automorphic factors $\kappa_{s, r}(\alpha, \tau)$ and $\mu_{s, r}(\alpha, \tau)$ by

$$
\alpha B(\tau)=B(\alpha \tau)\left(\overline{\mu_{s, r}(\alpha, \tau)}, \kappa_{s, r}(\alpha, \tau)\right) .
$$

We define a weight $\underline{k}$ of $\mathrm{U}(r, s)$ such that $\underline{k}=\left(c_{r+1, \sigma}, \ldots, c_{r+s, \sigma} ; c_{1, \sigma}, \ldots, c_{r, \sigma}\right)_{\sigma}$ with $c_{1, \sigma} \geq \cdots \geq c_{r, \sigma} \geq c_{r+1, \sigma}+r+s \geq \cdots \geq c_{r+s, \sigma}+r+s$. Using these we can develop the theory of holomorphic automorphic forms on $\mathrm{GU}(s, r)$ similar to the $\mathrm{GU}(r, s)$ case.

2C4. Embeddings of symmetric domains. We still follow [Shimura 1997]. Pick one Archimedean place. Write $z=\left(\begin{array}{l}x \\ y\end{array}\right) \in X_{r+1, s+1}, X_{r, s}$, and $w=\left(\begin{array}{l}u \\ v\end{array}\right) \in X_{s, r}$. We define the embeddings $\iota$ from $X_{r+1, s+1} \times X_{s, r}$ or $X_{r, s} \times X_{s, r}$ to $X_{r+s+1, r+s+1}$ or $X_{r+s, r+s}$ 
by

$$
\iota(z, w) \rightarrow\left(\begin{array}{ccc}
x & 0 & 0 \\
y & \frac{1}{2} \zeta & 0 \\
-\zeta^{-1} v^{*} y & -v^{*} & -u^{*}
\end{array}\right) .
$$

(The $\zeta$ really means the image of $\zeta$ at this Archimedean place.) Let $U=R T Q$, for

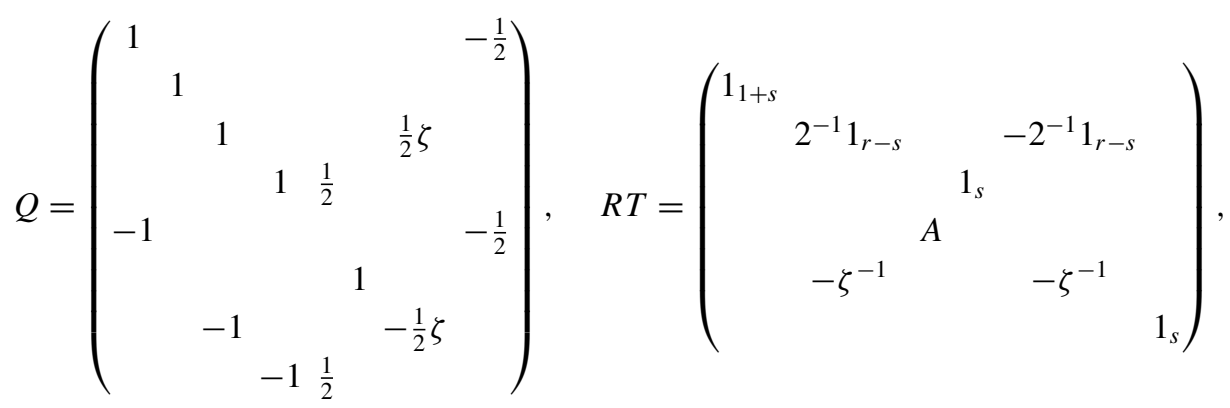

where $A=\left(\begin{array}{c}1 \\ 1_{s}\end{array}\right)$. (The $U$ here is the $U_{v}$ defined in [Shimura 1997, Section 22] and other notations are slightly different.) We also define $Q^{\prime}$ to be $Q$ with the second and sixth rows and columns (blockwise) deleted. Let

$$
R^{\prime} T^{\prime}=\left(\begin{array}{cccccc}
1_{s} & & & & \\
& 2^{-1} 1_{r-s} & & & -2^{-1} 1_{t} & \\
& & 1_{s} & & \\
& & A^{\prime} & & \\
& -\zeta^{-1} & & & -\zeta^{-1} & \\
& & & & & 1_{s}
\end{array}\right)
$$

with $A^{\prime}=1_{s}$. Define $U^{\prime}=R^{\prime} T^{\prime} Q^{\prime}$. Let $\wp(z)$ be the lower-right $r \times s$ block for $z \in X_{r+1, s+1}$ and $\iota_{U}(z, w)=\left(U^{-1} \iota(z, w)\right.$ as in [Shimura 1997, (22.2.1)]. If $z=\left(\begin{array}{l}x \\ y\end{array}\right)$ and $z_{1}=\left(\begin{array}{l}x_{1} \\ y_{1}\end{array}\right)$, let $\delta\left(z_{1}, z\right)=2^{s-r} \operatorname{det}\left[i\left(x_{1}^{*}-x\right)-y_{1}^{*} \theta^{-1} y\right]$. If we write $[h]_{S}$ for $S^{-1} h S$ then we have $\left[\operatorname{diag}\left(g, g_{1}\right)\right]_{S} \iota_{U}(z, w)=\iota_{U}\left(g z, g_{1} w\right),\left[\operatorname{diag}\left(g, g_{1}\right)\right]_{S^{\prime}} \iota_{U^{\prime}}(z, w)=$ $\iota_{U^{\prime}}\left(g z, g_{1} w\right)$ and

$$
j\left(\left[\operatorname{diag}\left(g, g_{1}\right)\right]_{S}, \iota_{U}(z, w)\right)=\delta(w, \wp(z))^{-1} \delta\left(g w, \wp\left(g_{1} z\right)\right) \operatorname{det}(\gamma) \overline{j_{g}(w)} j_{g_{1}}(z) .
$$

For a function $g$ on $X_{r+s+1, r+s+1}$ or $X_{r+s, r+s}$, we define the pullback $g^{\circ}$ to be the function on $X_{r+1, s+1} \times X_{s, r}$ or $X_{r, s} \times X_{s, r}$ given by

$$
g^{\circ}(z, w)=\delta(w, \wp(z))^{-k} g\left(\iota_{U}(z, w)\right) .
$$

Definition 2.5. We define a scalar weight $\kappa$ of $\mathrm{U}(s, r)$ to be the weight

$$
(\underbrace{-\kappa, \ldots,-\kappa}_{s} ; \underbrace{0, \ldots, 0}_{r}) \text {. }
$$


2D. Shimura varieties and Igusa varieties. Fix a neat open compact subgroup $K$ of $\mathrm{GU}^{0}(r, s)\left(\mathbb{A}_{f}\right)$ whose $p$-component is $\mathrm{GU}^{0}(r, s)\left(\mathbb{Z}_{p}\right)$; we refer to [Hsieh 2014] for the definitions and arithmetic models of Shimura varieties over the reflex field $E$, which we denote by $S_{G}(K)$. It parameterizes isomorphism classes of the quadruples $\left(A, \lambda, \iota, \bar{\eta}^{(\square)}\right) / S$, where $\square$ is a finite set of primes, $(A, \lambda)$ is a polarized abelian variety over some base ring $S, \lambda$ is an orbit (see [Hsieh 2014, Definition 2.1]) of prime-to- $\square$ polarizations of $A, \iota$ is an embedding of $O_{\mathscr{K}}$ into the endomorphism ring of $A$, and $\bar{\eta}^{(\square)}$ is some prime-to- $\square$ level structure of $A$. To each point $(\tau, g) \in X^{+} \times G\left(\mathbb{A}_{F, f}\right)$, we attach a quadruple as follows:

- The abelian variety $\mathscr{A}_{g}(\tau):=V \otimes_{\mathbb{Q}} \mathbb{R} / M_{[g]}\left(M_{[g]}:=H_{1}\left(\mathscr{A}_{g}(\tau), \hat{\mathbb{Z}}^{p}\right)\right)$.

- The polarization of $\mathscr{A}$ is given by the pullback of $-\langle,\rangle_{r, s}$ on $\mathbb{C}^{r, s}$ to $V \otimes \mathbb{Q} \mathbb{R}$ via $p(\tau)$.

- The complex multiplication $\iota$ is the $\mathscr{O}_{\mathscr{K}}$-action induced by the action on $V$.

- The prime-to- $p$ level structure $\eta_{g}^{(p)}: M \otimes \hat{\mathbb{Z}}^{p} \simeq M_{[g]}$ is defined by $\eta_{g}^{(p)}(x)=g * x$ for $x \in M$.

We have a similar theory for Shimura varieties for $\mathrm{GU}(s, r)$ as well.

There is also a theory of compactifications of $S_{G}(K)$, developed in [Lan 2008]. We let $\bar{S}_{G}(K)$ be a fixed choice of a toroidal compactification and $S_{G}^{*}(K)$ the minimal compactification.

We define some level groups at $p$, as in [Hsieh 2014, Section 1.10]. Recall that $G\left(\mathbb{A}_{f}\right) \supseteq K=\prod_{V} K_{v}$ is an open compact subgroup such that $K_{p}=G\left(\mathbb{Z}_{p}\right)$ and let $\Sigma$ be a finite set of primes including all primes above $p$ such that $K_{v}$ is spherical for all $v \notin \Sigma$. If we write $g_{p}=\left(\begin{array}{ll}A & B \\ C & D\end{array}\right)$ for the $p$-component of $g$, then define

$$
\begin{aligned}
K^{n} & =\left\{g \in K \mid g_{p} \equiv\left(\begin{array}{cc}
1_{r} & * \\
0 & 1_{s}
\end{array}\right) \bmod p^{n}\right\}, \\
K_{1}^{n} & =\left\{g \in K \mid A \in N_{r}\left(\mathbb{Z}_{p}\right) \bmod p^{n}, D \in N_{s}^{-}\left(\mathbb{Z}_{p}\right) \bmod p^{n}, C=0\right\}, \\
K_{0}^{n} & =\left\{g \in K \mid A \in B_{r}\left(\mathbb{Z}_{p}\right) \bmod p^{n}, D \in B_{s}^{-}\left(\mathbb{Z}_{p}\right) \bmod p^{n}, C=0\right\} .
\end{aligned}
$$

Now we recall briefly the notion of Igusa schemes over $\mathcal{O}_{v_{0}}$ (the localization of the integer ring of the reflex field at the $p$-adic place $v_{0}$ determined by $\iota_{p}: \mathbb{C} \simeq \mathbb{C}_{p}$ ) in [Hsieh 2014, Section 2]. Let $V$ be the Hermitian space for $\mathrm{U}(r, s), M$ be a standard lattice of $V$ and $M_{p}=M \otimes_{\mathbb{Z}} \mathbb{Z}_{p}$. Let $\operatorname{Pol}_{p}=\left\{N^{-1}, N^{0}\right\}$ be a polarization of $M_{p}$. The Igusa variety $I_{G}\left(K^{n}\right)$ of level $p^{n}$ is the scheme representing the usual quadruple for a Shimura variety together with

$$
j: \mu_{p^{n}} \otimes_{\mathbb{Z}} N^{0} \hookrightarrow A\left[p^{n}\right]
$$


where $A$ is the abelian variety in the quadruple. Note that the existence of $j$ implies that if $p$ is nilpotent in the base ring then $A$ must be ordinary. For any integer $m>0$, let $\mathrm{O}_{m}:=\mathfrak{O}_{v_{0}} / p^{m}$.

Igusa schemes over $\bar{S}_{G}(K)$. To define $p$-adic automorphic forms one needs Igusa schemes over $\bar{S}_{G}(K)$. We fix such a toroidal compactification and refer to [Hsieh 2014, Section 2.7.6] for the construction. We still denote it by $I_{G}\left(K^{n}\right)$. Then, over $\mathrm{O}_{m}, I_{G}\left(K^{n}\right)$ is a Galois covering of the ordinary locus of the Shimura variety with Galois group $\prod_{v \mid p} \mathrm{GL}_{r}\left(\mathcal{O}_{F, v} / p^{n}\right) \times \mathrm{GL}_{s}\left(\mathscr{O}_{F, v} / p^{n}\right)$. We write $I_{G}\left(K_{0}^{n}\right)=$ $I_{G}\left(K^{n}\right)^{K_{0}^{n}}$ and $I_{G}\left(K_{1}^{n}\right)=I_{G}\left(K^{n}\right)^{K_{1}^{n}}$ over $\mathcal{O}_{m}$.

Cusps. Let $1 \leq t \leq s$. We let $P_{t}$ be the maximal parabolic subgroup of $\mathrm{GU}(r, s)$ consisting of matrices which, in the block form with respect to $t+(r+s-2 t)+t$, are of the form

$$
\left(\begin{array}{ccc}
x & x & x \\
& x & x \\
& & x
\end{array}\right) .
$$

Let $G_{P_{t}}$ be the unitary similitude group with respect to the skew-Hermitian space for $\zeta$. Let $Y_{t}$ be the $\mathcal{O}_{\mathscr{K}}$ span of $\left\{y^{1}, \ldots, y^{t}\right\}$. We define the set of cusp labels by

$$
C_{t}(K):=\left(\mathrm{GL}\left(Y_{t}\right) \times G_{P_{t}}\left(\mathbb{A}_{f}\right)\right) N_{P_{t}}\left(\mathbb{A}_{f}\right) \backslash G\left(\mathbb{A}_{f}\right) / K .
$$

This is a finite set. We write $[g]$ for the class represented by $g \in G\left(\mathbb{A}_{f}\right)$. For each such $g$ whose $p$-component is 1 , we define $K_{P_{t}}^{g}=G_{P_{t}}\left(\mathbb{A}_{f}\right) \cap g K g^{-1}$ and write $S_{[g]}:=S_{G_{P_{t}}}\left(K_{P_{t}}^{g}\right)$ the corresponding Shimura variety for the group $G_{P}$ with level group $K_{P_{t}}^{g}$ ). By the strong approximation we can choose a set $\underline{C}_{t}(K)$ of representatives of $C_{t}(K)$ consisting of elements $g=p k^{0}$ for $p \in P_{t}\left(\mathbb{A}_{f}^{\Sigma}\right)$ and $k^{0} \in K^{0}$ for $K^{0}$ the spherical compact subgroup.

Definition 2.6 ( $p$-adic cusps). As in [Hsieh 2014], each pair $(g, h) \in C_{t}(K) \times H\left(\mathbb{Z}_{p}\right)$ can be regarded as a $p$-adic cusp, i.e., cusps of the Igusa tower.

Igusa schemes for unitary groups. We refer to [Hsieh 2014, Section 2.5] for the notion of Igusa schemes for the unitary groups $\mathrm{U}(r, s)$ (not the similitude group). It parameterizes quintuples $\left(A, \lambda, \iota, \bar{\eta}^{(p)}, j\right)_{/ S}$ similar to the Igusa schemes for unitary similitude groups but requiring $\lambda$ to be a prime-to- $p$ polarization of $A$ (instead of an orbit). In order to use the pullback formula algebraically we need a map of Igusa schemes, given by

$$
\begin{aligned}
i\left(\left[\left(A_{!}, \lambda_{!}, \iota_{1}, \eta_{!}^{p} K_{!}, j_{1}\right)\right],\left[\left(A_{2}, \lambda_{2}, \iota_{2}, \eta_{2}^{p} K_{2}, j_{2}\right)\right]\right) & \\
= & {\left[\left(A_{1} \times A_{2}, \lambda_{1} \times \lambda_{2}, \iota_{1}, \iota_{2},\left(\eta_{1}^{p} \times \eta_{2}^{p}\right) K_{3}, j_{1} \times j_{2}\right)\right] . }
\end{aligned}
$$


Similar to [Hsieh 2014], we know that, taking the change of polarization into consideration,

$$
i([z, g],[w, h])=[\iota(z, w),(g, h) \Upsilon]
$$

$(\Upsilon$ is as defined at the end of Section $2 B)$.

2D1. Geometric modular forms. Let $H=\prod_{v \mid p}\left(\mathrm{GL}_{r} \times \mathrm{GL}_{s}\right)$ and let $N \subset H$ be $\prod_{v \mid p}\left(N_{r} \times{ }^{t} N_{s}\right)$. To save notation we also write $H=\prod_{v \mid p} \mathrm{GL}_{r}\left(\mathbb{O}_{F, v}\right) \times \mathrm{GL}_{s}\left(\mathbb{O}_{F, v}\right)$ and let $N \subset H$ be $\prod_{v \mid p} N_{r}\left(\mathscr{O}_{F, v}\right) \times{ }^{t} N_{S}\left(\mathcal{O}_{F, v}\right)$. We define $\underline{\omega}=e^{*} \Omega_{\varphi_{G} / \bar{S}_{G}(K)}$ for $\Omega$ the sheaf of differentials on the universal semiabelian scheme $\mathscr{G}$ over the toroidal compactification (see [Hsieh 2014, Section 2.7.2] for a brief discussion). Recall that for $v \mid p$ we have $v=w \bar{w}$ in $\mathscr{K}$ with $w \in \Sigma_{p}$. Let $e_{w}$ and $e_{\bar{w}}$ be the corresponding projections for $\mathscr{K}_{v} \simeq \mathscr{K}_{w} \times \mathscr{K}_{\bar{w}}$; then $\underline{\omega}=e_{w} \underline{\omega} \oplus e_{\bar{w}} \underline{\omega}$. We also define

$$
\begin{aligned}
\mathscr{E}^{+} & :=\underline{\operatorname{Isom}}\left(0_{\bar{S}_{G}(K)}^{r}, e_{w} \underline{\omega}\right), \\
\mathscr{E}^{-} & :=\underline{\operatorname{Isom}}\left(\mathcal{O}_{\bar{S}_{G}(K)}^{s}, e_{\bar{w}} \underline{\omega}\right), \\
\mathscr{E} & :=\mathscr{E}^{+} \oplus \mathscr{E}^{-} .
\end{aligned}
$$

This is an $H$-torsor over $\bar{S}_{G}(K)$. We can define the automorphic sheaf $\omega_{\underline{k}}=\mathscr{E} \times{ }^{H} L_{\underline{k}}$. A section $f$ of $\underline{\omega}_{k}$ is a morphism $f: \mathscr{E} \rightarrow L_{\underline{k}}$ such that

$$
f(x, h \boldsymbol{w})=\rho_{\underline{k}}(h) f(x, \omega), \quad h \in H, x \in \bar{S}_{G}(K) .
$$

2E. p-adic automorphic forms on unitary groups. Let $R$ be a $p$-adic $\mathbb{Z}_{p}$-algebra and let $R_{m}:=R / p^{m}$. Let $T_{n, m}:=I_{G}\left(K^{n}\right)_{/ R_{m}}$. Define

$$
\begin{aligned}
V_{n, m} & =H^{0}\left(T_{n, m}, O_{T_{n, m}}\right), \\
V_{\underline{k}}\left(K_{\bullet}^{n}, R_{m}\right) & =H^{0}\left(T_{n, m / R_{m}}, \omega_{\underline{k}}\right)^{K_{\bullet}^{n} .}
\end{aligned}
$$

Let $V_{\infty, m}=\varliminf_{n} V_{n, m}$ and $V_{\infty, \infty}=\lim _{m} V_{\infty, m}$. Define $V_{p}(G, K):=V_{\infty, \infty}^{N}$, the space of $p$-adic modular forms. Let $\boldsymbol{T}=T\left(\mathbb{Z}_{p}\right) \subset H$ and let $\Lambda_{\boldsymbol{T}}:=\mathbb{Z}_{p} \llbracket \boldsymbol{T} \rrbracket$. The Galois action of $\boldsymbol{T}$ on $V_{\infty, m}^{N}$ makes the space of $p$-adic modular forms a discrete $\Lambda_{T}$-module.

Suppose $n \geq m$. To each $R_{m}$-quintuple $(\underline{A}, j)$ of level $K^{n}$, we can attach a canonical basis $\omega(j)$ of $H^{0}\left(A, \Omega_{A}\right)$. Therefore, we have a canonical isomorphism

$$
H^{0}\left(T_{n, m / R_{m}}, \omega_{\underline{k}}\right) \simeq V_{n, m} \otimes L_{\underline{k}}\left(R_{m}\right)
$$

given by

$$
f \mapsto \hat{f}(\underline{A}, j)=f(\underline{A}, j, \omega(j)) .
$$

We call $\hat{f}$ the $p$-adic avatar of $f$. 
Similarly, we can define an embedding of geometric modular forms into $p$-adic modular forms by

$$
f \mapsto \hat{f}(\underline{A}, j)=f(\underline{A}, \omega(j)) .
$$

We also define the morphism

$$
\beta_{\underline{k}}: V_{\underline{k}}\left(K_{1}^{n}, R_{m}\right) \rightarrow V_{n, m}^{N}, \quad f \mapsto \beta_{\underline{k}}(f):=l_{\underline{k}}(\hat{f}) .
$$

We can also pass to the limit for $m \rightarrow \infty$ to get the embedding of $V_{\underline{k}}\left(K_{1}^{n}, R\right)$ into $V_{\infty, \infty}^{N}$. We refer to [Hsieh 2014, Sections 3.8-3.9] for the definition of a $U_{p}$ Hecke operator and define Hida's ordinary projector

$$
e:=\lim _{n} U_{p}^{n !} .
$$

2F. Algebraic theory for Fourier-Jacobi expansions. We suppose $s>0$ in this subsection. Let $X_{t}^{\vee}=\operatorname{span}_{\mathscr{O}_{r}}\left\{x^{1}, \cdots, x^{t}\right\}$ and $Y_{t}=\operatorname{span}_{\mathrm{O}_{r}}\left\{y^{1}, \ldots, y^{t}\right\}$. Let $W_{t}$ be

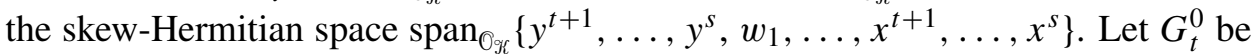
the unitary similitude group of $W_{t}$. Let $[g] \in C_{t}(K)$ and $K_{G_{P_{t}}}=G_{P_{t}}\left(\mathbb{A}_{f}\right) \cap g K^{-1}$ (we suppress the subscript $[g]$ so as to not make the notation too cumbersome). Let $\mathscr{A}_{t}$ be the universal abelian scheme over the Shimura variety $S_{G_{P_{t}}}\left(K_{G_{P_{t}}}\right)$. Write $g^{\vee}=k g_{i}^{\vee} \gamma$ for $\gamma \in G(F)^{+}$and $k \in K$. Define $X_{g}^{\vee}=X_{t}^{\vee} g_{i}^{\vee} \gamma$ and $Y_{g}=Y_{t} g_{i}^{\vee} \gamma$. Let $X_{g}=\left\{y \in\left(Y_{t} \otimes_{\mathbb{Q}} \mathbb{Z}\right) \cdot \gamma \mid\left\langle y, X_{g}^{\vee}\right\rangle \in \mathbb{Z}\right\}$. Then we have

$$
i: Y_{g} \hookrightarrow X_{g} .
$$

Let $\mathscr{L}_{[g]}$ be

$\underline{\operatorname{Hom}}_{\mathscr{O}}\left(X_{g}, \mathscr{A}_{t}^{\vee}\right) \times_{\underline{\operatorname{Hom}}_{\mathscr{Y}}\left(Y_{g}, A_{t}^{\vee}\right)}^{\underline{\operatorname{Hom}}_{\mathscr{O}}}\left(Y_{g}, \mathscr{A}_{t}\right)$

$$
:=\left\{\left(c, c^{t}\right) \mid c(i(y))=\lambda\left(c^{t}(y)\right), y \in Y_{g}\right\} .
$$

Here the $\underline{\text { Hom }}$ are the obvious sheaves over the big étale site of $S_{G_{P_{t}}}$, represented by abelian schemes. Let $\boldsymbol{c}$ and $\boldsymbol{c}^{\vee}$ be the universal morphisms over $\underline{\operatorname{Hom}}_{\mathcal{O}_{\mathcal{K}}}\left(X_{g}, \mathscr{A}_{t}^{\vee}\right)$ and $\underline{\operatorname{Hom}}_{\mathscr{O}}\left(Y_{g}, \mathscr{A}_{t}\right)$. Let $N_{P_{t}}$ be the unipotent radical of $P_{t}$ and $Z\left(N_{P_{t}}\right)$ be its center. Let $\overline{H_{[g]}}:=Z\left(N_{P_{t}}(F)\right) \cap g_{i} K g_{i}^{-1}$. Note that if we replace the components of $K$ at $v \mid p$ by $K_{1}^{n}$ then the set $H_{[g]}$ remains unchanged. Let $\Gamma_{[g]}:=\mathrm{GL}_{\mathscr{K}}\left(Y_{t}\right) \cap g_{i} K g_{i}^{-1}$. Let $\mathscr{P}_{\mathscr{A}_{t}}$ be the Poincaré sheaf over $\mathscr{A}_{t}^{\vee} \times \mathscr{A}_{t} / \mathscr{L}_{[g]}$ and $\mathscr{P}_{\mathscr{A}_{t}}^{\times}$its associated $\mathbb{G}_{m}$-torsor. Let $S_{[g]}:=\operatorname{Hom}\left(H_{[g]}, \mathbb{Z}\right)$. For any $h \in S_{[g]}$, let $c(h)$ be the tautological map $\mathscr{L}_{[g]} \rightarrow \mathscr{A}_{t}^{\vee} \times \mathscr{A}_{t}$ and $\mathscr{L}(h):=c(h)^{*} \mathscr{P}_{\mathscr{A}_{t}}$ its associated $\mathbb{G}_{m}$ torsor over $\mathscr{L}_{[g]}$.

It is well known (see [Lan 2008, Chapter 7], for example) that the minimal compactification $S_{G}^{*}(K)$ is the disjoint union of boundary components corresponding to each $t=1, \ldots, s$. Let $\mathbb{O}_{\mathbb{C}_{p}}$ be the valuation ring for $\mathbb{C}_{p}$. The following proposition is proved in [Lan 2008, Proposition 7.2.3.16]. Let $[g] \in C_{t}(K)$ and $\bar{x}$ be a $\mathscr{O}_{\mathbb{C}_{p}}$-point of the $t$-stratum of $S_{G}^{*}(K)(1 / E)$ corresponding to $[g]$. 
Proposition 2.7. Let $[\mathrm{g}]$ and $\bar{x}$ be as above. We write the subscript $\bar{x}$ to mean formal completion along $\bar{x}$. Let $\pi$ be the map $\bar{S}_{G}(K) \rightarrow S_{G}^{*}(K)$. Then $\pi_{*}\left(\mathcal{O}_{\bar{S}_{G}(K)}\right) \bar{x}$ is isomorphic to

$$
\left\{\sum_{h \in S_{[g]}^{+}} H^{0}\left(\mathscr{L}_{[g]}, \mathscr{L}(h)\right)_{\bar{x}} q^{h}\right\}^{\Gamma_{[g]}} .
$$

Here $S_{[g]}^{+}$means the totally nonnegative elements in $S_{[g]}$. The $q^{h}$ is just regarded as a formal symbol and $\Gamma_{[g]}$ acts on the set by a certain formula, which we omit.

For each $[g] \in C_{t}(K)$ we fix an $\bar{x}$ corresponding to it as above. Now we consider the diagram

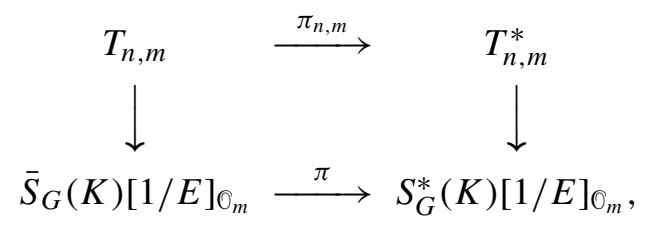

where $T_{n, m} \rightarrow T_{n, m}^{*} \rightarrow S_{G}^{*}(K)[1 / E]_{\bigcirc_{m}}$ is the Stein factorization. By [Lan 2013b, Corollary 6.2.2.8], $T_{n, m}^{*}$ is finite étale over $S_{G}^{*}(K)[1 / E]_{\bigcirc_{m}}$. Take a preimage of $\bar{x}$ in $T_{n, m}^{*}$, which we still denote by $\bar{x}$. (To do this, we have to extend the field of definition to include the maximal unramified extension of $L$.) Then the formal completion of the structure sheaf of $T_{n, m}^{*}$ and $S_{G}^{*}(K)[1 / E]_{\Theta_{m}}$ at $\bar{x}$ are isomorphic. So, for any $p$-adic automorphic form $f \in \lim _{m} \varliminf_{n} H^{0}\left(T_{n, m}, O_{n, m}\right)$ (with trivial coefficients), we have a Fourier-Jacobi coefficient

$$
\mathrm{FJ}(f) \in\left\{\prod_{h \in S_{[g]}^{+}} \underset{m}{\lim } \underset{n}{\lim } H^{0}\left(\mathscr{L}_{[g]}, \mathscr{L}(h)\right)_{\bar{x}} \cdot q^{h}\right\}_{[g]}
$$

by considering $f$ as a global section of $\pi_{n, m}^{*}\left(\mathcal{O}_{T_{n, m}}\right)=\mathcal{O}_{T_{n, m}^{*}}$ and pullback at the $\bar{x}$. Note that if $t=s=1$ then there is no need to choose the $\bar{x}$ and pullback, since the Shimura variety for $G_{t}$ is 0-dimensional (see [Hsieh 2014, (2.18)]). In application, when we construct families of Klingen Eisenstein series in terms of Fourier-Jacobi coefficients, we will take $t=1$ and define

$$
\mathscr{R}_{[g], \infty}:=\prod_{h \in S_{[g]}^{+}} \underset{m}{\lim } \underset{n}{\lim } H^{0}\left(\mathscr{L}_{[g]}, \mathscr{L}(h)\right)_{\bar{x}} \cdot q^{h} .
$$

We remark that the map FJ is injective on the space of forms with prescribed nebentypus at $p$ (this is not needed for our result though). This can be seen using the discussion in [Skinner and Urban 2014] right before Section 6.2 (which in turn uses result of Hida [2011] about the irreducibility of Igusa towers for the group $\mathrm{SU}(r, s) \subset \mathrm{U}(r, s)$, the kernel of the determinant map). In particular, to see this injectivity we need the fact that there is a bijection between the irreducible components 
of the generic and special fibers of $S_{G}^{*}(K)$ (see [Lan 2008, Section 6.4.1]) and that there is at least one cusp of any given genus on the ordinary locus of each irreducible component. (Note that the signature is $(r, s)$ for $r \geq s>0$ at all Archimedean places, so there is at least one cusp in $C_{t}(K)$ at each irreducible component. Since $p$ splits completely in $\mathscr{K}$, the cusps of minimal genus must be in the ordinary locus. On the other hand by the construction of minimal compactification the closure of the stratum of any genus $r$ is the union of all stratums of genus less than or equal to $r$. Note also that, since the geometric fibers of the minimal compactification are normal, their irreducible components are also connected components. This implies the existence of such a cusp on the ordinary locus.) See the Appendix for more details.

\section{Eisenstein series and Fourier-Jacobi coefficients}

The materials of this section are straightforward generalizations of parts of [Skinner and Urban 2014, Sections 9 and 11] and we use the same notations as there, so everything in this section should work out the same as [Skinner and Urban 2014] when specialized to the group $\mathrm{GU}(2,2) / \mathbb{Q}$.

3A. Klingen Eisenstein series. Let $\mathfrak{g} u(\mathbb{R})$ be the Lie algebra of $\mathrm{GU}(r, s)(\mathbb{R})$. Let $\delta$ be a character of the Klingen parabolic subgroup $P$ such that $\delta^{a+2 b+1}=\delta_{P}$ (the modulus character of $P$ ).

3A1. Archimedean picture. Let $v$ be an infinite place of $F$, so that $F_{v} \simeq \mathbb{R}$. Let $\boldsymbol{i}^{\prime}$ and $\boldsymbol{i}$ be the points on the Hermitian symmetric domain for $\mathrm{GU}(r, s)$ and $\mathrm{GU}(r+1, s+1)$ which are $\left(\begin{array}{c}i 1_{s} \\ 0\end{array}\right)$ and $\left(\begin{array}{c}i 1_{s+1} \\ 0\end{array}\right)$, respectively (here 0 means the $(r-s) \times s$ or $(r-s) \times(s+1)$ matrix 0$)$. Let $\mathrm{GU}(r, s)(\mathbb{R})^{+}$be the subgroup of $\mathrm{GU}(r, s)(\mathbb{R})$ whose similitude factor is positive. Let $K_{\infty}^{+}$and $K_{\infty}^{+, \prime}$ be the compact subgroups of $\mathrm{U}(r+1, s+1)(\mathbb{R})$ and $\mathrm{U}(r, s)(\mathbb{R})$ stabilizing $\boldsymbol{i}$ or $\boldsymbol{i}^{\prime}$, and let $K_{\infty}$ (resp. $\left.K_{\infty}^{\prime}\right)$ be the group generated by $K_{\infty}^{+}\left(\operatorname{resp} . K_{\infty}^{+, \prime}\right)$ and $\operatorname{diag}\left(1_{r+s+1},-1_{s+1}\right)$ (resp. $\operatorname{diag}\left(1_{r+s},-1_{s}\right)$ ).

Now let $(\pi, H)$ be a unitary tempered Hilbert representation of $\mathrm{GU}(r, s)(\mathbb{R})$ with $H_{\infty}$ the space of smooth vectors. We define a representation of $P(\mathbb{R})$ on $H_{\infty}$ as follows: for $p=m n$, where $n \in N_{P}(\mathbb{R})$ and $m=m(g, a) \in M_{P}(\mathbb{R})$ with $a \in \mathbb{C}^{\times}$, $g \in \mathrm{GU}(r+1, s+1)(\mathbb{R})$, put

$$
\rho(p) v:=\tau(a) \pi(g) v, \quad v \in H_{\infty} .
$$

We define a representation by smooth induction, $I\left(H_{\infty}\right):=\operatorname{Ind}_{P(\mathbb{R})}^{\mathrm{GU}(r+1, s+1)(\mathbb{R})} \rho$ and write $I(\rho)$ for the space of $K_{\infty}$-finite vectors in $I\left(H_{\infty}\right)$. For $f \in I(\rho)$ we also define, for each $z \in \mathbb{C}$, a function

$$
f_{z}(g):=\delta(m)^{(a+2 b+1) / 2+z} \rho(m) f(k), \quad g=m k \in P(\mathbb{R}) K_{\infty},
$$


and an action of $\mathrm{GU}(r+1, s+1)(\mathbb{R})$ on it by

$$
(\sigma(\rho, z)(g) f)(k):=f_{z}(k g) .
$$

Let $\left(\pi^{\vee}, V\right)$ be the irreducible $\left(\mathfrak{g u}(\mathbb{R}), K_{\infty}^{\prime}\right)$-module given by $\pi^{\vee}(x)=\pi\left(\eta^{-1} x \eta\right)$ for

$$
\eta=\left(\begin{array}{lll} 
& & 1_{b} \\
& 1_{a} & \\
-1_{b} & &
\end{array}\right)
$$

and $x$ in $\mathfrak{g u}(\mathbb{R})$ or $K_{\infty}^{\prime}$ (this does not mean the contragradient representation!). Let $\rho^{\vee}, I\left(\rho^{\vee}\right), I^{\vee}\left(H_{\infty}\right), \sigma\left(\rho^{\vee}, z\right)$ and $I\left(\rho^{\vee}\right)$ be the representations and spaces defined as above but with $\pi$ and $\tau$ replaced by $\pi^{\vee} \otimes(\tau \circ$ det $)$ and $\bar{\tau}^{c}$. We are going to define an intertwining operator. Let

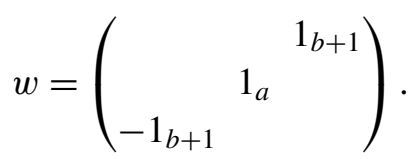

For any $z \in \mathbb{C}, f \in I\left(H_{\infty}\right)$ and $k \in K_{\infty}$, consider the integral

$$
A(\rho, z, f)(k):=\int_{N_{P}(\mathbb{R})} f_{z}(w n k) d n .
$$

This is absolutely convergent when $\operatorname{Re}(z)>\frac{1}{2}(a+2 b+1)$ and $A(\rho, z,-)$ in $\operatorname{Hom}_{C}\left(I\left(H_{\infty}\right), I^{\vee}\left(H_{\infty}\right)\right)$ intertwines the actions of $\sigma(\rho, z)$ and $\sigma\left(\rho^{\vee},-z\right)$.

Suppose $\pi$ is the holomorphic discrete series representation associated to the (scalar) weight $(0, \ldots, 0 ; \kappa, \ldots, \kappa)$; then it is well known that there is a unique (up to scalar) vector $v \in \pi$ such that $k \cdot v=\operatorname{det} \mu(k, i)^{-\kappa}$ (here $\mu$ means the second component of the automorphic factor $J$ instead of the similitude character) for any $k \in K_{\infty}^{+, \prime}$. Then, by the Frobenius reciprocity law, there is a unique (up to scalar) vector $\tilde{v} \in I(\rho)$ such that $k \cdot \tilde{v}=\operatorname{det} \mu(k, i)^{-\kappa} \tilde{v}$ for any $k \in K_{\infty}^{+}$. We fix $v$ and multiply $\tilde{v}$ by a constant so that $\tilde{v}(1)=v$. In $\pi^{\vee}, \pi(w) v$ has the action of $K_{\infty}^{+}$ given by multiplying by $\operatorname{det} \mu(k, i)^{-\kappa}$. We define $w^{\prime} \in \mathrm{U}(r+1, s+1)$ by

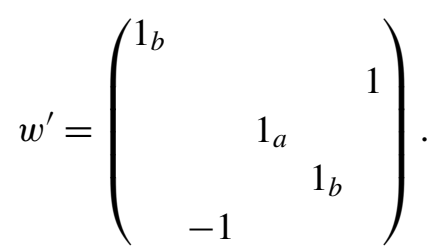

There is a unique vector $\tilde{v}^{\vee} \in I\left(\rho^{\vee}\right)$ such that the action of $K_{\infty}^{+}$is given by $\operatorname{det} \mu(k, i)^{-\kappa}$ and $\tilde{v}^{\vee}\left(w^{\prime}\right)=\pi(w) v$. Then, by uniqueness, there is a constant $c(\rho, z)$ such that $A(\rho, z, \tilde{v})=c(\rho, z) \tilde{v}^{\vee}$.

Definition 3.1. We define $F_{\kappa} \in I(\rho)$ to be the $\tilde{v}$ as above. 
3A2. Prime-to-p picture. Our discussion here follows [Skinner and Urban 2014, $\S 9.1 .2]$. Let $(\pi, V)$ be an irreducible, admissible representation of $\operatorname{GU}(r, s)\left(F_{v}\right)$ which is unitary and tempered. Let $\psi$ and $\tau$ be unitary characters of $\mathscr{K}_{v}^{\times}$such that $\psi$ is the central character for $\pi$. We define a representation $\rho$ of $P\left(F_{v}\right)$ as follows. For $p=m n$, where $n \in N_{P}\left(F_{v}\right)$ and $m=m(g, a) \in M_{P}\left(F_{v}\right)$ with $a \in K_{v}^{\times}$ and $g \in \mathrm{GU}\left(F_{v}\right)$, let

$$
\rho(p) v:=\tau(a) \pi(g) v, \quad v \in V .
$$

Let $I(\rho)$ be the representation defined by admissible induction, that is, $I(\rho)=$ $\operatorname{Ind}_{P\left(F_{v}\right)}^{\mathrm{GU}(r+1, s+1)\left(F_{v}\right)} \rho$. As in the Archimedean case, for each $f \in I(\rho)$ and each $z \in \mathbb{C}$ we define a function $f_{z}$ on $\mathrm{GU}(r+1, s+1)\left(F_{v}\right)$ by

$$
f_{z}(g):=\delta(m)^{(a+2 b+1) / 2+z} \rho(m) f(k) \text { for } g=m k \in P\left(F_{v}\right) K_{v}
$$

and a representation $\sigma(\rho, z)$ of $\mathrm{GU}(r+1, s+1)\left(F_{v}\right)$ on $I(\rho)$ by

$$
(\sigma(\rho, z)(g) f)(k):=f_{z}(k g) .
$$

Let $\left(\pi^{\vee}, V\right)$ be given by $\pi^{\vee}(g)=\pi\left(\eta^{-1} g \eta\right)$. This representation is also tempered and unitary. We denote by $\rho^{\vee}, I\left(\rho^{\vee}\right)$ and $\left(\sigma\left(\rho^{\vee}, z\right), I\left(\rho^{\vee}\right)\right)$ the representations and spaces defined as above but with $\pi$ and $\tau$ replaced by $\pi^{\vee} \otimes(\tau \circ$ det $)$ and $\bar{\tau}^{c}$, respectively.

For $f \in I(\rho), k \in K_{v}$ and $z \in \mathbb{C}$, consider the integral

$$
A(\rho, z, v)(k):=\int_{N_{P}\left(F_{v}\right)} f_{z}(w n k) d n .
$$

As a consequence of our hypotheses on $\pi$ this integral converges absolutely and uniformly for $z$ and $k$ in compact subsets of $\left\{z \mid \operatorname{Re}(z)>\frac{1}{2}(a+2 b+1)\right\} \times K_{v}$. Moreover, for such $z, A(\rho, z, f) \in I\left(\rho^{\vee}\right)$, and the operator $A(\rho, z,-) \in \operatorname{Hom}_{\mathbb{C}}\left(I(\rho), I\left(\rho^{\vee}\right)\right)$ intertwines the actions of $\sigma(\rho, z)$ and $\sigma\left(\rho^{\vee},-z\right)$.

For any open subgroup $U \subseteq K_{v}$, let $I(\rho)^{U} \subseteq I(\rho)$ be the finite-dimensional subspace consisting of functions satisfying $f(k u)=f(k)$ for all $u \in U$. Then the function

$$
\left\{z \in \mathbb{C} \mid \operatorname{Re}(z)>\frac{1}{2}(a+2 b+1)\right\} \rightarrow \operatorname{Hom}_{\mathbb{C}}\left(I(\rho)^{U}, I\left(\rho^{\vee}\right)^{U}\right), \quad z \mapsto A(\rho, z,-)
$$

is holomorphic. This map has a meromorphic continuation to all of $\mathbb{C}$.

We finally remark that, when $\pi$ and $\tau$ are unramified, there is a unique (up to scalar) unramified vector $F_{\rho_{v}} \in I(\rho)$.

3A3. Global picture. We follow [Skinner and Urban 2014, §9.1.4]. Let ( $\pi, V)$ be an irreducible, cuspidal, tempered automorphic representation of $\mathrm{GU}(r, s)\left(\mathbb{A}_{F}\right)$. This is an admissible $\left(\mathfrak{g u}(\mathbb{R}), K_{\infty}^{\prime}\right)_{v \mid \infty} \times \mathrm{GU}(r, s)\left(\mathbb{A}_{f}\right)$-module which is a restricted tensor product of local irreducible admissible representations. Let $\psi, \tau: \mathbb{A}_{\mathscr{K}}^{\times} \rightarrow \mathbb{C}^{\times}$ 
be Hecke characters such that $\psi$ is the central character of $\pi$. Let $\tau=\otimes \tau_{w}$ and $\psi=\otimes \psi_{w}$ be their local decompositions, $w$ running over places of $F$. Define a representation of $\left(P\left(F_{\infty}\right) \cap K_{\infty}\right) \times P\left(\mathbb{A}_{F, f}\right)$ by putting

$$
\rho(p) v:=\bigotimes\left(\rho_{w}\left(p_{w}\right) v_{w}\right),
$$

Let $I(\rho)$ be the restricted product $\otimes I\left(\rho_{w}\right)$ with respect to the $F_{\rho_{w}}$ at those $w$ at which $\tau_{w}, \psi_{w}$ and $p i_{w}$ are unramified. As before, for each $z \in \mathbb{C}$ and $f \in I(\rho)$, we define a function $f_{z}$ on $\mathrm{GU}(r+1, s+1)\left(\mathbb{A}_{F}\right)$ as

$$
f_{z}(g):=\bigotimes f_{w, z}\left(g_{w}\right)
$$

where $f_{w, z}$ are defined as before, and an action $\sigma(\rho, z)$ of

$$
\left(\mathfrak{g u}, K_{\infty}\right) \otimes \mathrm{GU}(r+1, s+1)\left(\mathbb{A}_{f}\right)
$$

on $I(\rho)$ by $\sigma(\rho, z):=\bigotimes \sigma\left(\rho_{w}, z\right)$. Similarly, we define $\rho^{\vee}, I\left(\rho^{\vee}\right)$ and $\sigma\left(\rho^{\vee}, z\right)$, but with the corresponding things replaced by their $\vee$, and we have global versions of the intertwining operators $A(\rho, f, z)$.

Definition 3.2. Let $\Sigma$ be a finite set of primes of $F$ containing all the infinite places, primes dividing $p$, and places where $\pi$ or $\tau$ is ramified. Then we call the triple $\mathscr{D}=(\pi, \tau, \Sigma)$ an Eisenstein datum.

3A4. Klingen-type Eisenstein series on $G$. We follow [Skinner and Urban 2014, $\S 9.1 .5]$ in this subsubsection. Let $\pi, \psi$ and $\tau$ be as above. For $f \in I(\rho)$ and $z \in \mathbb{C}$, there are maps from $I(\rho)$ and $I\left(\rho^{\vee}\right)$ to spaces of automorphic forms on $P\left(\mathbb{A}_{F}\right)$ given by

$$
f \longmapsto\left(g \mapsto f_{z}(g)(1)\right) .
$$

In the following we often write $f_{z}$ for the automorphic form on $P\left(\mathbb{A}_{F}\right)$ given by this recipe.

If $g \in \mathrm{GU}(r+1, s+1)\left(\mathbb{A}_{F}\right)$, it is well known that

$$
E(f, z, g):=\sum_{\gamma \in P(F) \backslash G(F)} f_{z}(\gamma g)
$$

converges absolutely and uniformly for $(z, g)$ in compact subsets of

$$
\left\{z \in \mathbb{C} \mid \operatorname{Re}(z)>\frac{1}{2}(a+2 b+1)\right\} \times \mathrm{GU}(r+1, s+1)\left(\mathbb{A}_{F}\right) .
$$

Therefore, we get some automorphic forms which are called Klingen Eisenstein series.

Definition 3.3. For any parabolic subgroup $R$ of $\mathrm{GU}(r+1, s+1)$ and an automorphic form $\varphi$, we define $\varphi_{R}$ to be the constant term of $\varphi$ along $R$, defined 
by

$$
\varphi_{R}(g)=\int_{n \in N_{R}(F) \backslash N_{R}\left(\mathbb{A}_{F}\right)} \varphi(n g) d n .
$$

The following lemma is well known (see [Skinner and Urban 2014, Lemma 9.2]).

Lemma 3.4. Let $R$ be a standard $F$-parabolic subgroup of $\mathrm{GU}(r+1, s+1)$ (i.e, $R \supseteq B$, where $B$ is the standard Borel subgroup). Suppose $\operatorname{Re}(z)>\frac{1}{2}(a+2 b+1)$. Then:

(i) If $R \neq P$ then $E(f, z, g)_{R}=0$.

(ii) $E(f, z,-)_{P}=f_{z}+A(\rho, f, z)_{-z}$.

\section{B. Siegel Eisenstein series on $G_{n}$.}

3B1. Local picture. Our discussion in this subsection follows [Skinner and Urban 2014, $\S 11.1-11.3]$ closely. Let $Q=Q_{n}$ be the Siegel parabolic subgroup of $\mathrm{GU}_{n}$ consisting of matrices $\left(\begin{array}{cc}A_{q} & B_{q} \\ 0 & D_{q}\end{array}\right)$. It consists of matrices whose lower-left $n \times n$ block is zero.

For a finite place $v$ of $F$ and a character $\chi$ of $\mathscr{K}_{v}^{\times}$, we let $I_{n}(\chi)$ be the space of smooth $K_{n, v}$-finite functions (here $K_{n, v}$ means the open compact group $G_{n}\left(\mathscr{O}_{F, v}\right)$ ) $f: K_{n, v} \rightarrow \mathbb{C}$ such that $f(q k)=\chi\left(\operatorname{det} D_{q}\right) f(k)$ for all $q \in Q_{n}\left(F_{v}\right) \cap K_{n, v}$, where we write $q$ as a block matrix $q=\left(\begin{array}{cc}A_{q} & B_{q} \\ 0 & D_{q}\end{array}\right)$. For $z \in \mathbb{C}$ and $f \in I(\chi)$, we also define a function $f(z,-): G_{n}\left(F_{v}\right) \rightarrow \mathbb{C}$ by $f(z, q k):=\chi\left(\operatorname{det} D_{q}\right)\left|\operatorname{det} A_{q} D_{q}^{-1}\right|_{v}^{z+n / 2} f(k)$ for $q \in Q_{n}\left(F_{v}\right)$ and $k \in K_{n, v}$.

For $f \in I_{n}(\chi), z \in \mathbb{C}$ and $k \in K_{n, v}$, the intertwining integral is defined by

$$
M(z, f)(k):=\bar{\chi}^{n}\left(\mu_{n}(k)\right) \int_{N_{Q_{n}}\left(F_{v}\right)} f\left(z, w_{n} r k\right) d r .
$$

For $z$ in compact subsets of $\left\{\operatorname{Re}(z)>\frac{1}{2} n\right\}$, this integral converges absolutely and uniformly, with the convergence being uniform in $k$. In this case it is easy to see that $M(z, f) \in I_{n}\left(\bar{\chi}^{c}\right)$. A standard fact from the theory of Eisenstein series says that this has a continuation to a meromorphic section on all of $\mathbb{C}$.

Let $U \subseteq \mathbb{C}$ be an open set. By a meromorphic section of $I_{n}(\chi)$ on $U$ we mean a function $\varphi: \mathcal{U} \rightarrow I_{n}(\chi)$ taking values in a finite-dimensional subspace $V \subset I_{n}(\chi)$ and such that $\varphi: \vartheta \rightarrow V$ is meromorphic.

For Archimedean places there is a similar picture (see [loc. cit.]).

3B2. Global picture. For an idele class character $\chi=\bigotimes \chi_{v}$ of $\mathbb{A}_{\mathscr{K}}^{\times}$, we define a space $I_{n}(\chi)$ to be the restricted tensor product defined using the spherical vectors $f_{v}^{\text {sph }} \in I_{n}\left(\chi_{v}\right), \quad f_{v}^{\text {sph }}\left(K_{n, v}\right)=1$, at the finite places $v$ where $\chi_{v}$ is unramified.

For $f \in I_{n}(\chi)$ we consider the Eisenstein series

$$
E(f ; z, g):=\sum_{\gamma \in Q_{n}(F) \backslash G_{n}(F)} f(z, \gamma g) .
$$


This series converges absolutely and uniformly for $(z, g)$ in compact subsets of $\left\{\operatorname{Re}(z)>\frac{1}{2} n\right\} \times G_{n}\left(\mathbb{A}_{F}\right)$. The automorphic form defined is called Siegel Eisenstein series.

Let $\varphi: \mathcal{U} \rightarrow I_{n}(\chi)$ be a meromorphic section; then we put $E(\varphi ; z, g)=$ $E(\varphi(z) ; z, g)$. This is defined at least on the region of absolute convergence and it is well known that it can be meromorphically continued to all $z \in \mathbb{C}$.

Now, for $f \in I_{n}(\chi), z \in \mathbb{C}$ and $k \in \prod_{v \nmid \infty} K_{n, v} \prod_{v \mid \infty} K_{\infty}$, there is a similar intertwining integral $M(z, f)(k)$ as above, but with the integration being over $N_{Q_{n}}\left(\mathbb{A}_{F}\right)$. This again converges absolutely and uniformly for $z$ in compact subsets of $\left\{\operatorname{Re}(z)>\frac{1}{2} n\right\} \times K_{n}$. Thus, $z \mapsto M(z, f)$ defines a holomorphic section $\left\{\operatorname{Re}(z)>\frac{1}{2} n\right\} \rightarrow I_{n}\left(\bar{\chi}^{c}\right)$. This has a continuation to a meromorphic section on $\mathbb{C}$. For $\operatorname{Re}(z)>\frac{1}{2} n$, we have

$$
M(z, f)=\bigotimes_{v} M\left(z, f_{v}\right), \quad f=\bigotimes f_{v}
$$

The functional equation for Siegel Eisenstein series is

$$
E(f, z, g)=\chi^{n}(\mu(g)) E(M(z, f) ;-z, g),
$$

in the sense that both sides can be meromorphically continued to all $z \in \mathbb{C}$ and the equality is understood as of meromorphic functions of $z \in \mathbb{C}$.

3B3. The pullback formulas. Let $\chi$ be a unitary idele class character of $A_{\mathscr{K}}^{\times}$. Given a unitary, tempered, cuspidal eigenform $\varphi$ on $\operatorname{GU}(r, s)$ which is a pure tensor, we formally define the integral

$$
\begin{aligned}
& F_{\varphi}(f ; z, g):=\int_{\mathrm{U}(r, s)\left(\mathbb{A}_{F}\right)} f\left(z, S^{-1} \alpha\left(g, g_{1} h\right) S\right) \bar{\chi}\left(\operatorname{det} g_{1} g\right) \varphi\left(g_{1} h\right) d g_{1}, \\
& \quad f \in I_{r+s+1}(\chi), g \in \mathrm{GU}(r+1, s+1)\left(\mathbb{A}_{F}\right), h \in \mathrm{GU}(r, s)\left(\mathbb{A}_{F}\right), \mu(g)=\mu(h) .
\end{aligned}
$$

This is independent of $h$. (We suppress the $\chi$ in the notation for $F_{\varphi}$ since its choice is implicitly given by $f$.) We also formally define

$$
\begin{aligned}
F_{\varphi}^{\prime}(f ; z, g):= & \int_{\mathrm{U}(r, s)\left(\mathbb{A}_{F}\right)} f\left(z, S^{\prime-1} \alpha\left(g, g_{1} h\right) S^{\prime}\right) \bar{\chi}\left(\operatorname{det} g_{1} g\right) \varphi\left(g_{1} h\right) d g_{1}, \\
& f \in I_{r+s}(\chi), g \in \mathrm{GU}(r, s)\left(\mathbb{A}_{F}\right), h \in \mathrm{GU}(r, s)\left(\mathbb{A}_{F}\right), \mu(g)=\mu(h) .
\end{aligned}
$$

The pullback formulas are the identities in the following proposition.

Proposition 3.5. Let $\chi$ be a unitary idele class character of $\mathbb{A}_{\mathscr{K}}^{\times}$.

(i) If $f \in I_{r+s}(\chi)$, then $F_{\varphi}(f ; z, g)$ converges absolutely and uniformly for $(z, g)$ in compact sets of $\{\operatorname{Re}(z)>r+s\} \times \mathrm{GU}(r, s)\left(\mathbb{A}_{F}\right)$ and, for any $h \in \mathrm{GU}(r, s)\left(\mathbb{A}_{F}\right)$ 
such that $\mu(h)=\mu(g)$,

$$
\begin{aligned}
\int_{\mathrm{U}(r, s)(F) \backslash \mathrm{U}(r, s)\left(\mathbb{A}_{F}\right)} E\left(f ; z, S^{\prime-1} \alpha\left(g, g_{1} h\right) S^{\prime}\right) \bar{\chi}\left(\operatorname{det} g_{1} h\right) \varphi\left(g_{1} h\right) & d g_{1} \\
& =F_{\varphi}^{\prime}(f ; z, g) .
\end{aligned}
$$

(ii) If $f \in I_{r+s+1}(\chi)$, then $F_{\varphi}(f ; z, g)$ converges absolutely and uniformly for $(z, g)$ in compact sets of $\left\{\operatorname{Re}(z)>r+s+\frac{1}{2}\right\} \times \mathrm{GU}(r+1, s+1)\left(\mathbb{A}_{F}\right)$ and, for any $h \in \mathrm{GU}(r, s)\left(\mathbb{A}_{F}\right)$ such that $\mu(h)=\mu(g)$,

$$
\begin{aligned}
\int_{\mathrm{U}(r, s)(F) \backslash \mathrm{U}(r, s)\left(\mathbb{A}_{F}\right)} E\left(f ; z, S^{-1} \alpha\left(g, g_{1} h\right) S\right) \bar{\chi}\left(\operatorname{det} g_{1} h\right) \varphi\left(g_{1} h\right) d g_{1} & \\
= & \sum_{\gamma \in P(F) \backslash G(r+1, s+1)(F)} F_{\varphi}(f ; z, \gamma g),
\end{aligned}
$$

with the series converging absolutely and uniformly for $(z, g)$ in compact subsets of $\left\{\operatorname{Re}(z)>r+s+\frac{1}{2}\right\} \times \mathrm{GU}(r+1, s+1)\left(\mathbb{A}_{F}\right)$.

Proof. The global integral $F_{\varphi}$ and $F_{\varphi}^{\prime}$ can be written as a product of local integrals. The absolute convergence of local integrals for $F_{\varphi}^{\prime}$ is proved in [Lapid and Rallis 2005, Lemma 2]. The absolute convergence for the global integral $F_{\varphi}^{\prime}$ follows from this and the explicit computations in [Lapid and Rallis 2005] at all unramified places, together with the assumption that $\varphi$ is tempered. The absolute convergence for $F_{\varphi}$ is proved in the same way. Then part (i) is proved by Piatetski-Shapiro and Rallis [Gelbart et al. 1987] and (ii) is a straightforward generalization by Shimura [1997], which is in turn due to earlier works of Garrett [1984; 1989]. Both are straightforward consequences of the double coset decompositions in [Shimura 1997, Propositions 2.4 and 2.7].

\section{C. Fourier-Jacobi expansion.}

3C1. Fourier-Jacobi expansion. We will usually write $e_{\mathbb{A}}(x)=e_{\mathbb{A}_{\mathbb{Q}}}\left(\operatorname{Tr}_{\mathbb{A}_{F} / \mathbb{A}_{\mathbb{Q}}} x\right)$ for $x \in \mathbb{A}_{F}$. For any automorphic form $\varphi$ on $\operatorname{GU}(r, s)\left(\mathbb{A}_{F}\right), \beta \in S_{m}(F)$ for $m \leq s$. We define the Fourier-Jacobi coefficient at $g \in \mathrm{GU}(r, s)\left(\mathbb{A}_{F}\right)$ as

$$
\varphi_{\beta}(g)=\int_{S_{m}(F) \backslash S_{m}\left(\mathbb{A}_{F}\right)} \varphi\left(\left(\begin{array}{cccc}
1_{s} & 0 & S & 0 \\
0 & 1_{r-s} & 0 \\
0 & 0 & 1_{s}
\end{array}\right) g\right) e_{\mathbb{A}}(-\operatorname{Tr}(\beta S)) d S .
$$

In fact, we are mainly interested in two cases: $m=s$, or $r=s$ and arbitrary $m \leq s$. In particular, suppose $G=G_{n}=\mathrm{GU}(n, n), 0 \leq m \leq n$ are integers, and $\beta \in S_{m}(F)$. Let $\varphi$ be a function on $G(F) \backslash G(\mathbb{A})$. The $\beta$-th Fourier-Jacobi coefficient $\varphi_{\beta}$ of $\varphi$ 
at $g$ is defined by

$$
\varphi_{\beta}(g):=\int \varphi\left(\left(\begin{array}{ccc}
1_{n} & S & 0 \\
& 0 & 0 \\
& 1_{n}
\end{array}\right) g\right) e_{\mathbb{A}}(-\operatorname{Tr} \beta S) d S .
$$

Now we prove a useful formula on the Fourier-Jacobi coefficients for Siegel Eisenstein series.

Definition 3.6. Put

$$
\begin{aligned}
& Z:=\left\{\left(\begin{array}{ccc}
1_{n} & z & 0 \\
0_{n} & 1_{n}
\end{array}\right) \mid z \in S_{m}(\mathscr{K})\right\} \\
& V:=\left\{\left(\begin{array}{ccc}
1_{m} x & z & y \\
1_{n-m} & y^{*} & 0 \\
& 1_{m} & \\
0_{n} & -x^{*} & 1_{n-m}
\end{array}\right) \mid x, y \in M_{m(n-m)}(\mathscr{K}), z-x y^{*} \in S_{m}(\mathscr{K})\right\} \text {, } \\
& X:=\left\{\left(\begin{array}{ccc}
1_{m} x & \multicolumn{1}{c}{0_{n}} \\
1_{n-m} & \multicolumn{1}{c}{} \\
0_{n} & -x_{m}^{*} & 1_{n-m}
\end{array}\right) \mid x \in M_{m(n-m)}(\mathscr{K})\right\} \\
& Y:=\left\{\left(\begin{array}{ccc}
1_{n} & z & y \\
& y^{*} & 0 \\
0_{n} & 1_{n}
\end{array}\right) \mid y \in M_{m(n-m)}(\mathcal{K})\right\} .
\end{aligned}
$$

From now on we will usually write $w_{n}$ for $\left({ }_{-1_{n}} 1_{n}\right)$.

Proposition 3.7. Let $f$ be in $I_{n}(\tau)$ and suppose $\beta \in S_{m}(F)$ is totally positive. If $E(f ; z, g)$ is the Siegel Eisenstein series on $\mathrm{GU}_{n}$ defined by $f$ for some $\operatorname{Re}(z)$ sufficiently large, then the $\beta$-th Fourier-Jacobi coefficient $E_{\beta}(f ; z, g)$ satisfies

$$
\begin{aligned}
& E_{\beta}(f ; z, g) \\
& =\sum_{\gamma \in Q_{n-m}(F) \backslash \mathrm{GU}_{n-m}(F)} \sum_{y \in Y} \int_{S_{m}(\mathbb{A})} f\left(w_{n}\left(\begin{array}{ccc}
1_{n} & t \bar{y} & 0 \\
& 1_{n}
\end{array}\right) \alpha_{n-m}(1, \gamma) g\right) e_{\mathbb{A}}(-\operatorname{Tr} \beta S) d S,
\end{aligned}
$$

where

$$
\alpha_{n-m}(\gamma)=\left(\begin{array}{cccc}
1 & & & \\
& D & & C \\
& & 1 & \\
& B & & A
\end{array}\right)
$$

if $g_{1}=\left(\begin{array}{ll}A & B \\ C & D\end{array}\right)$, where $A, B, C$ and $D$ are $(n-m) \times(n-m)$ matrices. 
Proof. We follow [Ikeda 1994, Section 3]. Let $H$ be the normalizer of $V$ in $G$. Then

$$
G_{n}(F)=\bigsqcup_{i=1}^{m} Q_{n}(F) \xi_{i} H(F)
$$

for

$$
\xi_{i}:=\left(\begin{array}{llll}
0_{m-i} & 0 & -1_{m-i} & 0 \\
0 & 1_{n-m+i} & 0 & 0 \\
1_{m-i} & 0 & 0_{m-i} & 0 \\
0 & 0_{n-m+i} & 0 & 1_{n-m+i}
\end{array}\right) .
$$

Unfolding the Eisenstein series, we get

$$
\begin{array}{r}
E_{\beta}(f ; z, g)=\sum_{i>0} \sum_{\gamma \in Q_{n}(F) \backslash Q_{n}(F) \xi_{i} H(F)} \int f\left(\gamma\left(\begin{array}{ccc}
1_{n} & S & 0 \\
& 0 & 0 \\
& 1_{n}
\end{array}\right) g\right) e_{\mathbb{A}}(-\operatorname{Tr}(\beta S)) d S \\
+\sum_{\gamma \in Q_{n}(F) \backslash Q_{n}(F) \xi_{0} H(F)} \int f\left(\gamma\left(\begin{array}{ccc}
1_{n} & S & 0 \\
& 0 & 0 \\
1_{n}
\end{array}\right) g\right) e_{\mathbb{A}}(-\operatorname{Tr}(\beta S)) d S .
\end{array}
$$

By [Ikeda 1994, Lemma (3.1)] (see [ibid., p. 628]), the first term vanishes. Also, we have [loc. cit.]

$$
\begin{aligned}
Q_{n}(F) \backslash Q_{n}(F) \xi_{0} H(F) & =\xi_{0} Z(F) X(F) Q_{n-m}(F) \backslash G_{n-m}(F) \\
& =\xi_{0} X(F) \cdot Q_{n-m}(F) \backslash G_{n-m}(F) \cdot Z(F) \\
& =w_{n} Y(F) S_{m}(F) w_{n-m} Q_{n-m}(F) \backslash G_{n-m}(F)
\end{aligned}
$$

(note that $S_{m}$ commutes with $X$ and $G_{n-m}$ ). So

$$
E_{\beta}(f ; z, g)=\sum_{\gamma \in Q_{n-m}(F) \backslash G_{n-m}(F)} \sum_{y \in Y(F)} \int_{S_{m}(\mathbb{A})} f\left(w _ { n } \left(\begin{array}{ccc}
1_{n} & \left.\left.\begin{array}{cc}
t \bar{y} & y \\
& 1_{n}
\end{array}\right) \alpha_{n-m}(1, \gamma) g\right) \\
& \times e_{\mathbb{A}}(-\operatorname{Tr}(\beta S)) d S
\end{array}\right.\right.
$$

Note that the final integral is essentially a product of local ones.

Now we record some useful formulas:

Definition 3.8. If $g_{v} \in \mathrm{U}_{n-m}\left(F_{v}\right)$ and $x \in \mathrm{GL}_{m}\left(\mathscr{K}_{v}\right)$, then define $\mathrm{FJ}_{\beta}\left(f_{v} ; z, y, g, x\right)$

$$
=\int_{S_{m}\left(F_{v}\right)} f\left(w_{n}\left(\begin{array}{ccc}
1_{n} & S & y \\
& t \bar{y} & 0 \\
& 1_{n}
\end{array}\right) \alpha\left(\operatorname{diag}\left(x,{ }^{t} \bar{x}^{-1}\right), g\right)\right) e_{F_{v}}(-\operatorname{Tr} \beta S) d S,
$$


where

$$
\alpha\left(g_{1}, g_{2}\right)=\left(\begin{array}{cccc}
A & & B & \\
& D^{\prime} & & C^{\prime} \\
C & & D & \\
& B^{\prime} & & A^{\prime}
\end{array}\right) \quad \text { if } g_{1}=\left(\begin{array}{ll}
A & B \\
C & D
\end{array}\right), g_{2}=\left(\begin{array}{ll}
A^{\prime} & B^{\prime} \\
C^{\prime} & D^{\prime}
\end{array}\right) .
$$

We also define

$$
f_{v, \beta, z}(g):=f\left(z, w_{n}\left(\begin{array}{cc}
1_{n} & S \\
& 1_{n}
\end{array}\right) g\right) e_{v}(-\operatorname{Tr} \beta S) d S .
$$

Since

$$
\left(\begin{array}{ccc}
1_{n} & S & X \\
& { }^{t} \bar{X} & \\
& & 1_{n}
\end{array}\right)\left(\begin{array}{ccccc}
1_{m} & & & \\
& \bar{A}^{-1} & & \\
& & 1_{m} & \\
& B \bar{A}^{-1} & & A
\end{array}\right)=\left(\begin{array}{cccc}
1_{m} & X B \bar{A}^{-1} & & \\
& \bar{A}^{-1} & & \\
& & 1_{m} & \\
& B \bar{A}^{-1} & & A
\end{array}\right)\left(\begin{array}{cccc}
1_{n} & S-X B^{t} \bar{X} & X A \\
& \bar{A}^{t} \bar{X} & \\
& & & \\
& & &
\end{array}\right.
$$

it follows that:

$$
\begin{aligned}
\mathrm{FJ}_{\beta}(f ; z, X, & \left.\left(\begin{array}{cc}
A & B \bar{A}^{-1} \\
& \bar{A}^{-1}
\end{array}\right) g, Y\right) \\
& =\tau_{v}^{c}(\operatorname{det} A)^{-1}|\operatorname{det} A \bar{A}|_{v}^{z+n / 2} e_{v}\left(-\operatorname{Tr}\left({ }^{t} \bar{X} \beta X B\right)\right) \mathrm{FJ}_{\beta}(f ; z, X A, g, Y) .
\end{aligned}
$$

Also, we have

$$
\mathrm{FJ}_{\beta}(f ; z, y, g, x)=\tau_{v}(\operatorname{det} x)|\operatorname{det} x \bar{x}|_{\mathbb{A}}^{-(z+n / 2-m)} \mathrm{FJ}_{t_{\bar{x}} \beta x}\left(f ; z, x^{-1} y, g, 1\right) .
$$

3C2. Weil representations. We define the Weil representations which will be used in calculating local Fourier-Jacobi coefficients in the next section.

The local set-up. Let $v$ be a place of $F$. Let $h \in S_{m}\left(F_{v}\right)$, det $h \neq 0$. Let $\mathrm{U}_{h}$ be the unitary group of this metric and denote by $V_{v}$ the corresponding Hermitian space. Let $V_{n-m}:=\mathscr{K}_{v}^{(n-m)} \oplus \mathcal{K}_{v}^{(n-m)}:=X_{v} \oplus Y_{v}$ be the skew-Hermitian space associated to $\mathrm{U}(n-m, n-m)$. Let $W:=V_{v} \otimes \mathscr{K}_{v} V_{n-m, v}$. Then $(-,-):=$ $\operatorname{Tr}_{\mathscr{K}_{v} / F_{v}}\left(\langle-,-\rangle_{h} \otimes \mathscr{K}_{v}\langle-,-\rangle_{n-m}\right)$ is a $F_{v}$ linear pairing on $W$ that makes $W$ into a $4 m(n-m)$-dimensional symplectic space over $F_{v}$. The canonical embedding of $\mathrm{U}_{h} \times \mathrm{U}_{n-m}$ into $\mathrm{Sp}(W)$ realizes the pair $\left(\mathrm{U}_{h}, \mathrm{U}_{n-m}\right)$ as a dual pair in $\operatorname{Sp}(W)$. Let $\lambda_{v}$ be a character of $\mathscr{K}_{v}^{\times}$such that $\left.\lambda_{v}\right|_{F_{v}^{\times}}=\chi_{\mathscr{K} / F, v}^{m}$. It is well known (see [Kudla 1994]) that there is a splitting $\mathrm{U}_{h}\left(F_{v}\right) \times \mathrm{U}_{n-m}\left(F_{v}\right) \hookrightarrow \mathrm{Mp}\left(W, F_{v}\right)$ of the metaplectic cover $\operatorname{Mp}\left(W, F_{v}\right) \rightarrow \operatorname{Sp}\left(W, F_{v}\right)$ determined by the character $\lambda_{v}$. This gives the Weil representation $\omega_{h, v}(u, g)$ of $\mathrm{U}_{h}\left(F_{v}\right) \times \mathrm{U}_{n-m}\left(F_{v}\right)$, where $u \in \mathrm{U}_{h}\left(F_{v}\right)$ and $g \in \mathrm{U}_{n-m}\left(F_{v}\right)$, via the Weil representation of $\operatorname{Mp}\left(W, F_{v}\right)$ on the space of Schwartz functions $\mathscr{S}\left(V_{v} \otimes_{\mathscr{K}_{v}} X_{v}\right)$. Moreover, we write $\omega_{h, v}(g)$ to mean $\omega_{h, v}(1, g)$. For $X \in M_{m \times(n-m)}\left(\mathscr{K}_{v}\right)$, we define $\langle X, X\rangle_{h}:={ }^{t} \bar{X} \beta X$ (note this is an $(n-m) \times(n-m)$ 
matrix). We record here some useful formulas for $\omega_{h, v}$ which are generalizations of the formulas in [Skinner and Urban 2014, Section 10]:

- $\omega_{h, v}(u, g) \Phi(X)=\omega_{h, v}(1, g) \Phi\left(u^{-1} X\right)$.

- $\omega_{h, v}\left(\operatorname{diag}\left(A,{ }^{t} \bar{A}^{-1}\right)\right) \Phi(X)=\lambda(\operatorname{det} A)|\operatorname{det} A|_{\mathscr{\ell}} \Phi(X A)$.

- $\omega_{h, v}(r(S)) \Phi(x)=\Phi(x) e_{v}\left(\operatorname{Tr}\langle X, X\rangle_{h} S\right)$.

- $\omega_{h, v}(\eta) \Phi(x)=|\operatorname{det} h|_{v} \int \Phi(Y) e_{v}\left(\operatorname{Tr}_{\mathscr{K}_{v} / F_{v}}\left(\operatorname{Tr}\langle Y, X\rangle_{h}\right)\right) d Y$.

Global setup. Let $h \in S_{m}(F)$ be positive definite. We can define global versions of $\mathrm{U}_{h}, \mathrm{GU}_{h}, X, Y, W$, and $(-,-)$, analogously to the local case. Fixing an idele class character $\lambda=\otimes \lambda_{v}$ of $\mathbb{A}_{\mathscr{K}}^{\times} / \mathscr{F}^{\times}$such that $\left.\lambda\right|_{F^{\times}}=\chi_{\mathscr{K} / F}^{m}$, the associated local splitting described above then determines a global splitting $\mathrm{U}_{h}\left(\mathbb{A}_{F}\right) \times \mathrm{U}_{1}\left(\mathbb{A}_{F}\right) \hookrightarrow \mathrm{Mp}\left(W, \mathbb{A}_{F}\right)$ and hence an action $\omega_{h}:=\otimes \omega_{h, v}$ of $\mathrm{U}_{h}\left(\mathbb{A}_{F}\right) \times \mathrm{U}_{1}\left(\mathbb{A}_{F}\right)$ on the Schwartz space $\mathscr{S}\left(V_{\mathrm{A}_{\mathcal{K}}} \otimes_{\mathscr{K}} X\right)$.

\section{Local computations}

In this section we do the local computations for Klingen Eisenstein sections realized as the pullbacks of Siegel Eisenstein sections. We will compute the Fourier and Fourier-Jacobi coefficients for the Siegel sections and the pullback Klingen Eisenstein sections.

4A. Archimedean computations. Let $v$ be an Archimedean place of $F$.

4A1. Fourier coefficients.

Definition 4.1. $\quad f_{\kappa, n}(z, g)=J_{n}\left(g, i 1_{n}\right)^{-\kappa}\left|J_{n}\left(g, i 1_{n}\right)\right|^{\kappa-2 z-n}$.

Now we recall [Skinner and Urban 2014, Lemma 11.4]. Let $J_{n}\left(g, i 1_{n}\right):=$ $\operatorname{det}\left(C_{g} i 1_{n}+D_{g}\right)$ for $g=\left(\begin{array}{cc}A_{g} & B_{g} \\ C_{g} & D_{g}\end{array}\right)$.

Lemma 4.2. Suppose $\beta \in S_{n}(\mathbb{R})$. Then the function $z \mapsto f_{\kappa, \beta}(z, g)$ has a meromorphic continuation to all of $\mathbb{C}$. Furthermore, if $\kappa \geq n$ then $f_{\kappa, n, \beta}(z, g)$ is holomorphic at $z_{\kappa}:=\frac{1}{2}(\kappa-n)$ and, for $y \in \mathrm{GL}_{n}(\mathbb{C}), f_{\kappa, n, \beta}\left(z_{\kappa}, \operatorname{diag}\left(y,{ }^{t} \bar{y}^{-1}\right)\right)=0$ if $\operatorname{det} \beta \leq 0$, while, if $\operatorname{det} \beta>0$, then

$$
\begin{aligned}
& f_{\kappa, n, \beta}\left(z_{\kappa}, \operatorname{diag}\left(y,{ }^{t} \bar{y}^{-1}\right)\right) \\
& \quad=\frac{(-2)^{-n}(2 \pi i)^{n \kappa}(2 / \pi)^{n(n-1) / 2}}{\prod_{j=0}^{n-1}(\kappa-j-1) !} e_{v}\left(i \operatorname{Tr}\left(\beta y^{t} \bar{y}\right)\right) \operatorname{det}(\beta)^{\kappa-n} \operatorname{det} \bar{y}^{\kappa} .
\end{aligned}
$$

4A2. Pullback sections. Now we assume that our $\pi$ is the holomorphic discrete series representation associated to the (scalar) weight $(0, \ldots, 0 ; \kappa, \ldots, \kappa)$ and let $\varphi$ be the unique (up to scalar) vector such that the action of $K_{\infty}^{+, '}$ (see Section 3A) is given by det $\mu(k, i)^{-\kappa}$. Recall also that in Section 3A we defined the Klingen 
section $F_{\kappa}(z, g)$ (denoted $\left.F_{\kappa}\right)$. Recall we have defined $S$ and $S^{\prime}$ in equations (1) and (2). Let

$$
\boldsymbol{i}:=\left(\begin{array}{cccc}
\frac{1}{2} i 1_{b} & & \\
& i & & \\
& \frac{1}{2} \zeta & \\
& & \frac{1}{2} i 1_{b}
\end{array}\right) \quad \text { or } \quad\left(\begin{array}{lll}
\frac{1}{2} i 1_{b} & & \\
& \frac{1}{2} \zeta & \\
& & \frac{1}{2} i 1_{b}
\end{array}\right)
$$

be the distinguished point in the symmetric domain for $\mathrm{GU}(n, n)$ or $\mathrm{GU}(n+1, n+1)$, for $n=a+2 b$. We define Archimedean sections to be

$$
f_{\kappa}(g)=J_{n+1}(g, \boldsymbol{i})^{-\kappa}\left|J_{n+1}(g, \mathbf{i})\right|^{\kappa-2 z-n-1}
$$

and

$$
f_{\kappa}^{\prime}(g)=J_{n}(g, \boldsymbol{i})^{-\kappa}\left|J_{n}(g, \mathbf{i})\right|^{\kappa-2 z-n}
$$

and the pullback sections on $\mathrm{GU}(a+b+1, b+1)$ and $\mathrm{GU}(a+b, a)$ to be

$$
F_{\kappa}(z, g):=\int_{\mathrm{U}(a+b, b)(\mathbb{R})} f_{\kappa}\left(z, S^{-1} \alpha\left(g, g_{1}\right) S\right) \bar{\tau}\left(\operatorname{det} g_{1}\right) \pi\left(g_{1}\right) \varphi d g_{1}
$$

and

$$
F_{\kappa}^{\prime}(z, g):=\int_{\mathrm{U}(a+b, b)(\mathbb{R})} f_{\kappa}^{\prime}\left(z, S^{\prime-1} \alpha\left(g, g_{1}\right) S^{\prime}\right) \bar{\tau}\left(\operatorname{det} g_{1}\right) \pi\left(g_{1}\right) \varphi d g_{1} \text {. }
$$

Lemma 4.3. The integrals $F_{\kappa}$ and $F_{\kappa}^{\prime}$ are absolutely convergent for $\operatorname{Re}(z)$ sufficiently large and, for such $z$, we have

(i) $F_{\kappa}(z, g)=c_{\kappa}(z) F_{\kappa, z}(g)$;

(ii) $F_{\kappa}^{\prime}(z, g)=c_{\kappa}^{\prime}(z) \pi(g) \varphi$;

where

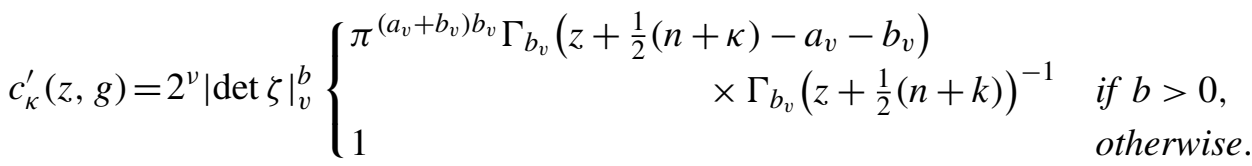

and $c_{\kappa}(z, g)=c_{\kappa}^{\prime}\left(z+\frac{1}{2}, g\right)$. Here $\Gamma_{m}(s):=\pi^{m(m+1) / 2} \prod_{k=0}^{m-1} \Gamma(s-k)$ and $v:=$ $(a+2 b) d b($ recall that $d=[F: \mathbb{Q}])$.

Proof. See [Shimura 1997, Propositions 22.2 and A2.9]. Note that the action of $(\beta, \gamma) \in \mathrm{U}(r, s) \times \mathrm{U}(s, r)$ is given by $\left(\beta^{\prime}, \gamma^{\prime}\right)$, defined there. Taking this into consideration, our conjugation matrix $S$ is Shimura's $S$ times $\Sigma^{-1}$ (with notation as there), which is defined in (22.1.2) in [Shimura 1997]. Also our result differs from [Skinner and Urban 2014, Lemma 11.6] by a power of 2, since we are using a different $S$ here. 
4A3. Fourier-Jacobi coefficients. We write $\mathrm{FJ}_{\beta, \kappa}$ for the Fourier-Jacobi coefficient defined in Definition 3.8 with $f_{v}$ chosen as $f_{\kappa, n}$.

Lemma 4.4. Let $z_{\kappa}=\frac{1}{2}(\kappa-n), \beta \in S_{m}(\mathbb{R}), m<n$ and $\operatorname{det} \beta>0$. Then:

(i) $\mathrm{FJ}_{\beta, \kappa}\left(z_{\kappa}, x, \eta, 1\right)=f_{\kappa, m, \beta}\left(z_{\kappa}+\frac{1}{2}(n-m), 1\right) e\left(i \operatorname{Tr}\left({ }^{t} \bar{X} \beta X\right)\right)$.

(ii) If $g \in U_{n-m}(\mathbb{R})$, then

$\mathrm{FJ}_{\beta, \kappa}\left(z_{\kappa}, X, g, 1\right)=e(i \operatorname{Tr} \beta) c_{m}(\beta, \kappa) f_{\kappa-m, n-m}\left(z_{\kappa}, g^{\prime}\right) w_{\beta}\left(g^{\prime}\right) \Phi_{\beta, \infty}(x)$,

where

$$
\begin{aligned}
g^{\prime} & =\left(\begin{array}{ll}
1_{n} & \\
& -1_{n}
\end{array}\right) g\left(\begin{array}{c}
1_{n} \\
1_{n}
\end{array}\right), \\
c_{t}(\beta, \kappa) & =\frac{(-2)^{-t}(2 \pi i)^{t \kappa}(2 / \pi)^{t(t-1) / 2}}{\prod_{j=0}^{t-1}(\kappa-j-1)} \operatorname{det} \beta^{\kappa-t}
\end{aligned}
$$

and $\Phi_{\beta, \infty}(x)=e^{-2 \pi \operatorname{Tr}\left(\langle x, x\rangle_{\beta}\right)}$.

Proof. Our proof is similar to [Skinner and Urban 2014, Lemma 11.5]. For (i) we first assume that $m \leq \frac{1}{2} n$; then there is a matrix $U \in \mathrm{U}_{n-m}$ such that $X U=(0, A)$ for $A$ an $m \times m$ positive semidefinite Hermitian matrix. It follows that $\mathrm{FJ}_{\beta, \kappa}(z, X, \eta, 1)=$ $\mathrm{FJ}_{\beta, \kappa}(z,(0, A), \eta, 1)$ and $e\left(i \operatorname{Tr}\left({ }^{t} \bar{X} \beta X\right)\right)=e\left(i \operatorname{Tr}\left(U^{-1}{ }^{t} \bar{X} \beta X U\right)\right)$, so we are reduced to the case when $X=(0, A)$.

Let $C$ be an $m \times m$ positive definite Hermitian matrix defined by $C=\sqrt{A^{2}+1}$. (Since $A$ is positive semidefinite Hermitian, this $C$ exists by linear algebra.) We have

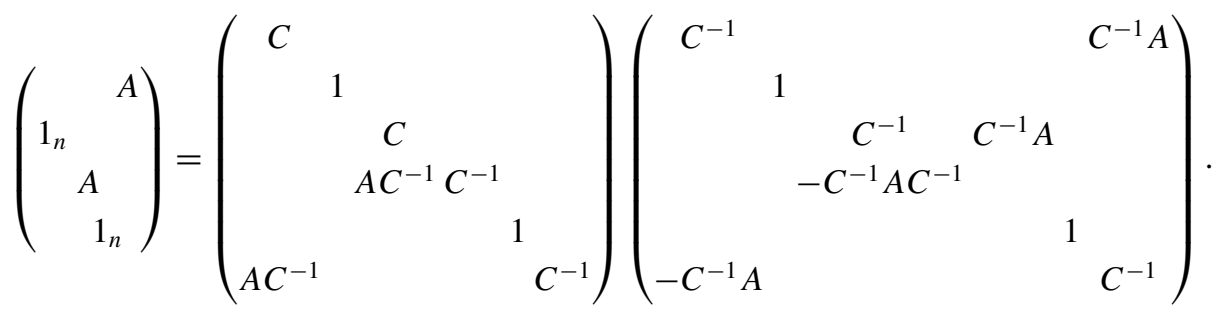

Write $k(A)$ for the second matrix in the right of the above, which belongs to $K_{n, \infty}^{+}$; then, as in [Skinner and Urban 2014, Lemma 11.5],

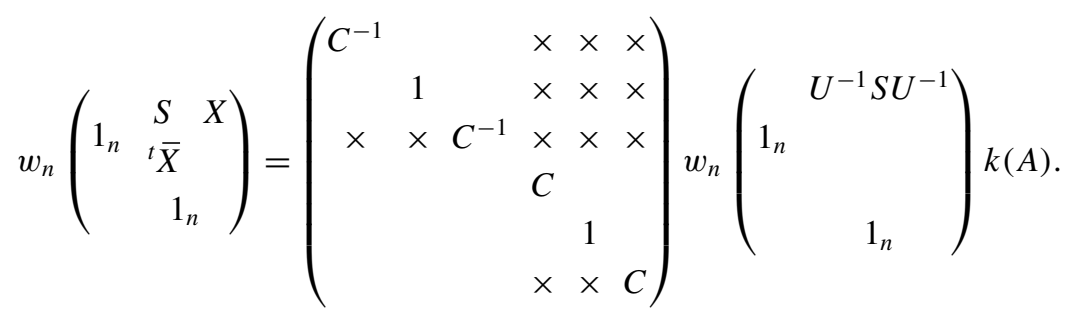


Thus,

$$
\begin{aligned}
\mathrm{FJ}_{\beta, \kappa}\left(z_{\kappa},(0, A), \eta, 1\right) & =(\operatorname{det} C)^{2 m-2 \kappa} \mathrm{FJ}_{\beta^{\prime}, \kappa}\left(z_{\kappa}, 0, \eta, 1\right), \beta^{\prime}=C \beta C \\
& =(\operatorname{det} C)^{2 m-2 \kappa} f_{\kappa, m, \beta^{\prime}}\left(z_{\kappa}+\frac{1}{2}(n-m), 1\right) \\
& =f_{\kappa, m, \beta}\left(z+\frac{1}{2}(n-m), 1\right) e(i \operatorname{Tr}(C \beta C-\beta)) .
\end{aligned}
$$

But

$$
\begin{aligned}
e(i \operatorname{Tr}(C \beta C-\beta))=e\left(i \operatorname{Tr}\left(C^{2} \beta-\beta\right)\right) & =e\left(i \operatorname{Tr}\left(\left(C^{2}-1\right) \beta\right)\right) \\
& =e\left(i \operatorname{Tr}\left(A^{2} \beta\right)\right)=e(i \operatorname{Tr}(A \beta A)) .
\end{aligned}
$$

This proves part (i).

Part (ii) is proved completely the same as [Skinner and Urban 2014, Lemma 11.5].

In the case when $m>\frac{1}{2} n$, we proceed similarly as in [Skinner and Urban 2014, Lemma 11.5], replacing $a$ and $u$ there by corresponding block matrices just as above. We omit the details.

\section{B. Finite primes, unramified case.}

4B1. Pullback integrals.

Lemma 4.5. Suppose $\pi, \psi$ and $\tau$ are unramified and $\varphi \in \pi$ is a new vector. If $\operatorname{Re}(z)>\frac{1}{2}(a+b)$ then the pullback integral converges and

$$
F_{\varphi}\left(f_{v}^{\mathrm{sph}} ; z, g\right)=\frac{L\left(\tilde{\pi}, \bar{\tau}^{c}, z+1\right)}{\prod_{i=0}^{a+2 b-1} L\left(2 z+a+2 b+1-i, \bar{\tau}^{\prime} \chi_{\mathscr{K}}^{i}\right)} F_{\rho, z}(g),
$$

where $F_{\rho, z}$ is the spherical section taking value $\varphi$ at the identity and

$$
F_{\varphi}\left(f_{v}^{\mathrm{sph}} ; z, g\right)=\frac{L\left(\tilde{\pi}, \bar{\tau}^{c}, z+\frac{1}{2}\right)}{\prod_{i=0}^{a+2 b-1} L\left(2 z+a+2 b-i, \bar{\tau}^{\prime} \chi_{\mathscr{K}}^{i}\right)} \pi(g) \varphi .
$$

This is computed in [Lapid and Rallis 2005, Proposition 3.3].

4B2. Fourier-Jacobi coefficients. Let $v$ be a prime of $F$ not dividing $p$ and $\tau$ a character of $\mathscr{K}_{v}^{\times}$. For $f \in I_{n}(\tau)$ and $\beta \in S_{m}\left(F_{v}\right), 0 \leq m \leq n$, we define the local Fourier-Jacobi coefficient to be

$$
f_{\beta}(z ; g):=\int_{S_{m}\left(F_{v}\right)} f\left(z, w_{n}\left(\begin{array}{ccc}
1_{n} & S & 0 \\
& 0 & 0 \\
& 1_{n}
\end{array}\right) g\right) e_{v}(-\operatorname{Tr} \beta S) d S .
$$

We first record straightforward generalizations of [Skinner and Urban 2014, Lemmas 11.7 and 11.8] to any fields [Shimura 1997, Propositions 18.14 and 19.2]. 
Lemma 4.6. Let $\beta \in S_{n}\left(F_{v}\right)$ and let $r:=\operatorname{rank}(\beta)$. Then, for $y \in \mathrm{GL}_{n}\left(\mathscr{K}_{v}\right)$,

$f_{v, \beta}^{\mathrm{sph}}\left(z, \operatorname{diag}\left(y,{ }^{t} \bar{y}^{-1}\right)\right)=\tau(\operatorname{det} y)|\operatorname{det} y \bar{y}|_{v}^{-z+n / 2} D_{v}^{-n(n-1) / 4}$

$$
\times \frac{\prod_{i=r}^{n-1} L\left(2 z+i-n+1, \bar{\tau}^{\prime} \chi_{\mathscr{K}}^{i}\right)}{\prod_{i=0}^{n-1} L\left(2 z+n-i, \bar{\tau}^{\prime} \chi_{\mathscr{K}}^{i}\right)} h_{v,{ }_{\bar{y}} \beta y}\left(\bar{\tau}^{\prime}(\varpi) q_{v}^{-2 z-n}\right),
$$

where $h_{v,{ }^{t} \beta y} \in \mathbb{Z}[X]$ is a monic polynomial depending on $v$ and ${ }^{t} \bar{y} \beta y$ but not on $\tau$. If $\beta \in S_{n}\left(\mathcal{O}_{F, v}\right)$ and $\operatorname{det} \beta \in \mathbb{O}_{F, v}^{\times}$, then we say that $\beta$ is $v$-primitive and, in this case, $h_{v, \beta}=1$.

Lemma 4.7. Suppose $v$ is unramified in $\mathscr{K}$. Let $\beta \in S_{m}\left(F_{v}\right)$ with $\operatorname{det} \beta \neq 0$ and let $\beta \in S_{m}\left(\mathcal{O}_{F_{v}}\right)$ and let $\lambda$ be an unramified character of $\mathscr{K}_{v}^{\times}$such that $\left.\lambda\right|_{F_{v}^{\times}}=1$. If $\beta \in \mathrm{GL}_{m}\left(\mathcal{O}_{F_{v}}\right)$ then, for $u \in \mathrm{U}_{\beta}\left(F_{v}\right)$,

$$
\mathrm{FJ}_{\beta}\left(f_{n}^{\mathrm{sph}} ; z, x, g, u\right)=\tau(\operatorname{det} u)|\operatorname{det} u \bar{u}|_{v}^{-z+1 / 2} \frac{f_{n-m}^{\mathrm{sph}}(z, g) \omega_{\beta}(u, g) \Phi_{0}(x)}{\prod_{i=0}^{m-1} L\left(2 z+n-i, \bar{\tau}^{\prime} \chi_{\mathscr{K}}^{i}\right)} .
$$

\section{C. Prime-to-p ramified case.}

4C1. Pullback integrals. Again let $v$ be a prime of $F$ not dividing $p$. We fix some $x$ and $y$ in $\mathscr{K}$ which are divisible by some high power of $\varpi_{v}$ (which can be made precise from the proof of the following two lemmas). (When we are moving things $p$-adically, the $x$ and $y$ are not going to change.) We define $f^{\dagger} \in I_{n+1}(\tau)$ to be the Siegel section supported on the cell $Q\left(F_{v}\right) w_{a+2 b+1} N_{Q}\left(\mathscr{O}_{F, v}\right)$, where $w_{a+2 b+1}=\left({ }_{-1_{a+2 b+1}} 1_{a+2 b+1}\right)$ and the value at $N_{Q}\left(\mathscr{O}_{F, v}\right)$ equals 1 . Similarly, we define $f^{\dagger, \prime} \in I_{n}(\tau)$ to be the section supported in $Q\left(F_{v}\right) w_{a+2 b} N_{Q}\left(\mathbb{O}_{F, v}\right)$ that takes value 1 on $N_{Q}\left(0_{F, v}\right)$.

Definition 4.8. $\quad f_{v, \operatorname{sieg}}(g):=f^{\dagger}\left(g \tilde{S}_{v}^{-1} \tilde{\gamma}_{v}\right) \in I_{n+1}(\tau)$,

where $\tilde{\gamma}_{v}$ is defined to be

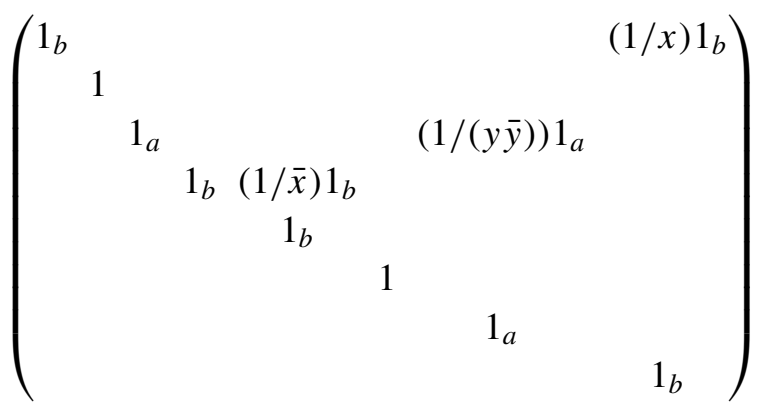


and

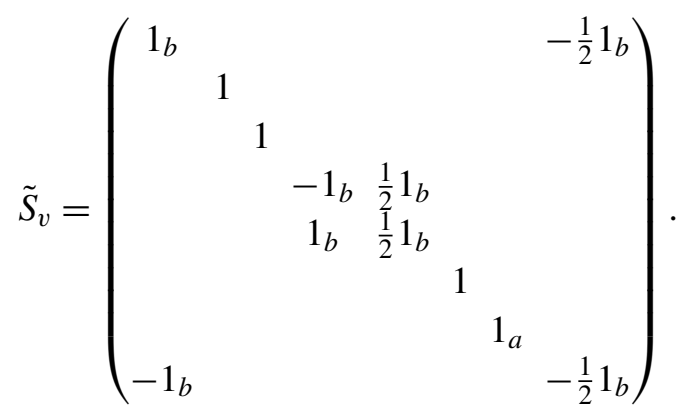

Similarly, we define $f_{v, \text { sieg }}^{\prime}(g):=f^{\dagger, \prime}\left(g \tilde{S}_{v}^{-1} \tilde{\gamma}_{v}^{\prime}\right)$ for

$$
\tilde{S}_{v}^{\prime}:=\left(\begin{array}{cccccc}
1_{b} & & & & & -\frac{1}{2} 1_{b} \\
& 1_{a} & & & & \\
& & -1_{b} & \frac{1}{2} 1_{b} & & \\
& & 1_{b} & \frac{1}{2} 1_{b} & & \\
& & & & 1_{a} & \\
-1_{b} & & & & & -\frac{1}{2} 1_{b}
\end{array}\right)
$$

and

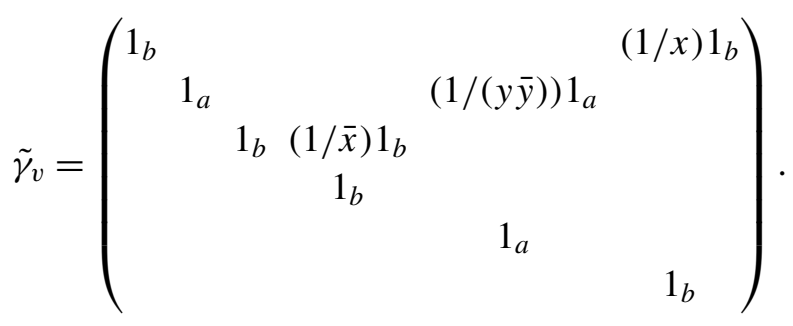

Lemma 4.9. Let $K_{v}^{(2)}$ be the subgroup of $G\left(F_{v}\right)$ of matrices of the form

$$
\left(\begin{array}{ccccc}
1_{b} & & & & d \\
a & 1 & f & b & c \\
& & 1_{a} & & g \\
& & & 1_{b} & e \\
& & & & 1
\end{array}\right)
$$

where $e=-{ }^{t} \bar{a}, \quad b={ }^{t} \bar{d}, \quad g=-\zeta^{t} \bar{f}, \quad b \in M\left(\mathcal{O}_{v}\right), \quad c-f \zeta^{t} \bar{f} \in \mathcal{O}_{F, v}, a \in(x)$, $e \in(\bar{x}), f \in(y \bar{y})$ and $g \in(2 \zeta y \bar{y})$. Then $F_{\varphi}(z ; g, f)$ is supported in $P w K_{v}^{(2)}$ and is invariant under the action of $K_{v}^{(2)}$. 
Proof. Let $S_{x, y}$ consist of matrices

$$
S:=\left(\begin{array}{llll}
S_{11} & S_{12} & S_{13} & S_{14} \\
S_{21} & S_{22} & S_{23} & S_{24} \\
S_{31} & S_{32} & S_{33} & S_{34} \\
S_{41} & S_{42} & S_{43} & S_{44}
\end{array}\right)
$$

in the space of Hermitian $(a+2 b+1) \times(a+2 b+1)$ matrices (the blocks are with respect to the partition $b+1+a+b$ ) such that the entries of $S_{13}$ and $S_{23}$ (resp. $S_{14}$ and $S_{24}, S_{31}$ and $S_{32}, S_{41}$ and $S_{42}$ ) are divisible by $y$ (resp. $x, \bar{y}, \bar{x}$ ), while the entries of $S_{33}$ (resp. $S_{34}, S_{43}, S_{44}$ ) are divisible by $y \bar{y}$ (resp. $x \bar{y}, \bar{x} y, x \bar{x}$ ). Let $Q_{x, y}:=Q\left(F_{v}\right) \cdot\left(\begin{array}{c}1 \\ s_{x, y} 1\end{array}\right)$.

Write

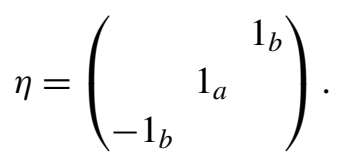

As in [Skinner and Urban 2014, Proposition 11.16], for

$$
g=\left(\begin{array}{lllll}
a_{1} & a_{2} & a_{3} & b_{1} & b_{2} \\
a_{4} & a_{5} & a_{6} & b_{3} & b_{4} \\
a_{7} & a_{8} & a_{9} & b_{5} & b_{6} \\
c_{1} & c_{2} & c_{3} & d_{1} & d_{2} \\
c_{4} & c_{5} & c_{6} & d_{3} & d_{4}
\end{array}\right),
$$

we have

$$
\begin{aligned}
\gamma(g, 1) \in \operatorname{supp} f_{v, \text { sieg }} & \Longleftrightarrow S_{v}^{-1} \alpha(g, 1) A w_{a+2 b+1} d_{x, y} \tilde{\gamma}^{-1} \in Q_{x, y} \\
& \Longleftrightarrow S_{v}^{-1} \alpha\left(g w, \eta \operatorname{diag}\left(\bar{x}^{-1}, 1, x\right)\right) A w^{\prime} d_{y} \tilde{\gamma}^{-1} \in Q_{x, y} .
\end{aligned}
$$

Here,

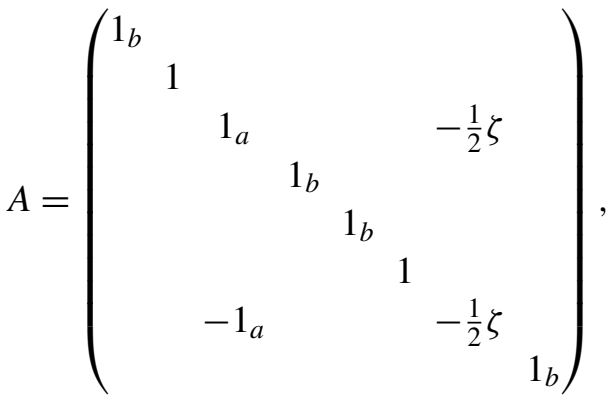

$$
\begin{aligned}
& d_{x, y}=\operatorname{diag}\left(1,1, y, x, 1,1, \bar{y}^{-1}, \bar{x}^{-1}\right), \\
& d_{y}=\operatorname{diag}\left(1,1, y, 1,1,1, \bar{y}^{-1}, 1\right),
\end{aligned}
$$




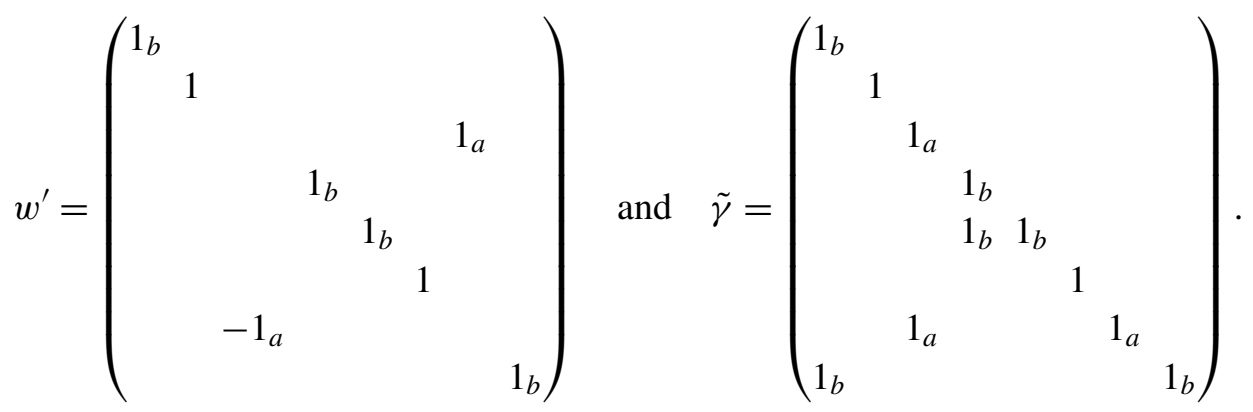

Here, $x$ and $y$ stand for the corresponding block matrices of the corresponding size. Recall that $\gamma\left(m\left(g_{1}, 1\right), g_{1}\right) \in Q$; by multiplying this to the left for $g_{1}=$ $\operatorname{diag}\left(\bar{x}, 1, x^{-1}\right) \eta^{-1}$, we are reduced to proving that, if $\gamma(g, 1) w^{\prime} d_{y} \tilde{\gamma}^{-1} \in Q_{x, y}$, then $g \in P w K_{v}^{(2)} w^{-1}$. A computation tells us that $\gamma(g, 1) w^{\prime} d_{y} \tilde{\gamma}^{-1}$ equals the product

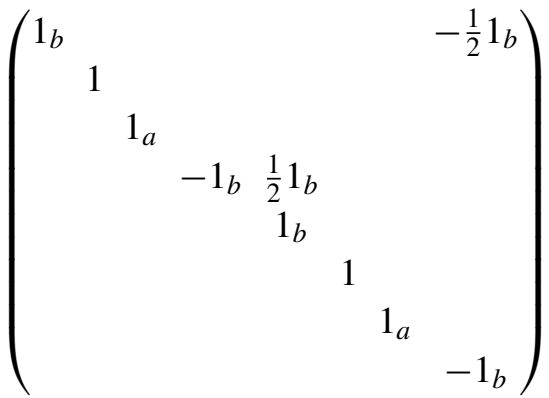

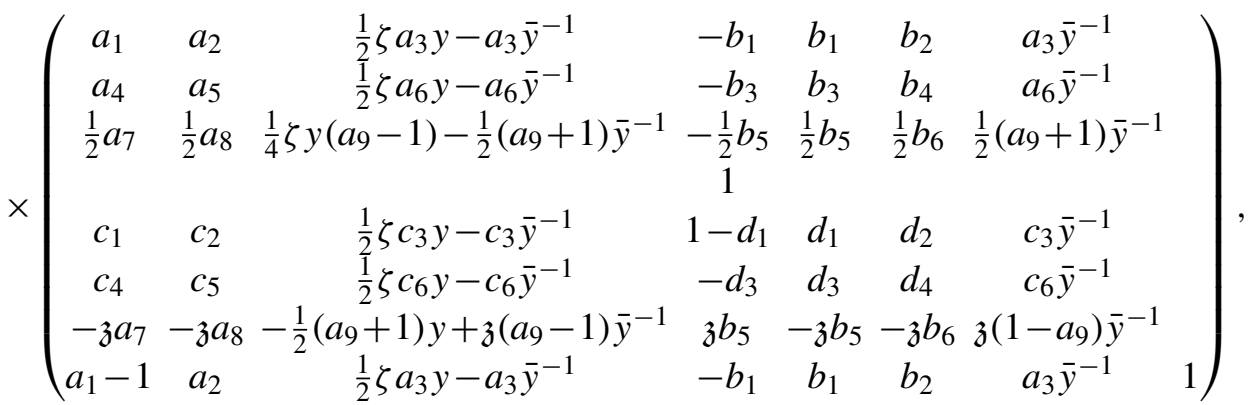

where $\mathfrak{z}=\zeta^{-1}$.

One first proves that $d_{4} \neq 0$ by looking at the second row of the lower left of the above matrix, so by left-multiplying $g$ by some matrix in $N_{P}$, we may assume that $d_{2}=b_{2}=b_{4}=b_{6}=0$, then the result follows by an argument similarly to the proof of Lemma 4.36 later on.

Now recall that

$$
g=\left(\begin{array}{lll}
a_{5} & a_{6} & a_{4} \\
a_{8} & a_{9} & a_{7} \\
a_{2} & a_{3} & a_{1}
\end{array}\right) .
$$


Let $\mathfrak{Y}$ be the set of $g$ such that the entries of $a_{2}$ are integers, the entries of $a_{3}$ (resp. $a_{1}-1,1-a_{5}, a_{6}, a_{4}, a_{8}, a_{7}$ ) are divisible by $y \bar{y}$ (resp. $\bar{x}, x, \bar{x} y, x \bar{x}$, $\left.\frac{1}{2} \bar{y} y \zeta, \bar{y} y x \zeta\right)$, and $1-a_{9}=y \bar{y} \zeta(1+y \bar{y} N)$ for some $N$ with integral entries.

Lemma 4.10. Let $\varphi_{x}=\pi\left(\operatorname{diag}\left(\bar{x}, 1, x^{-1}\right) \eta^{-1}\right) \varphi$, where $\varphi$ is invariant under the action of $\mathfrak{Y}$ defined above; then:

(i) $F_{\varphi_{x}}\left(f_{v, \text { sieg }} ; z, w\right)=\tau(y \bar{y} x)\left|(y \bar{y})^{2} x \bar{x}\right|_{v}^{-z-(a+2 b+1) / 2} \operatorname{Vol}(\mathfrak{Y}) \cdot \varphi$.

(ii) $F_{\varphi_{x}}^{\prime}\left(f_{v, \text { sieg }}^{\prime} ; z, w\right)=\tau(y \bar{y} x)\left|(y \bar{y})^{2} x \bar{x}\right|_{v}^{-z-(a+2 b) / 2} \operatorname{Vol}(\mathfrak{Y}) \cdot \varphi$.

Proof. First, one computes

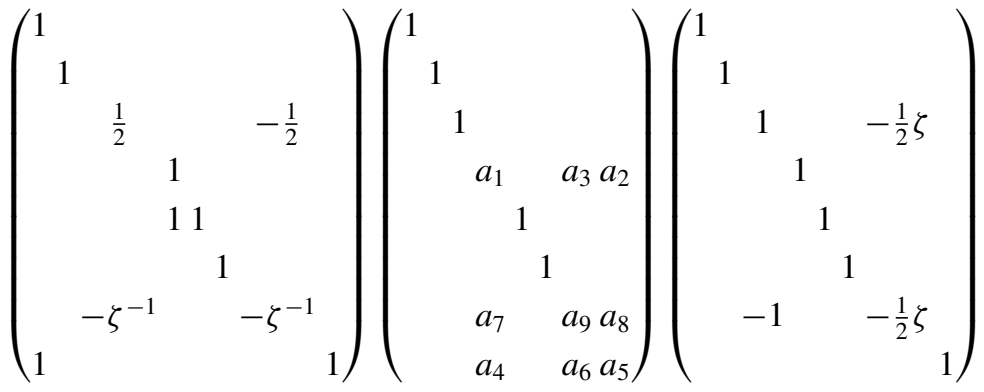

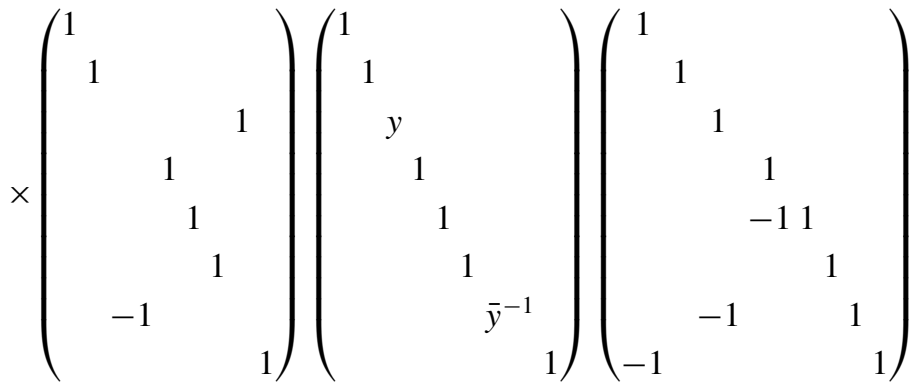

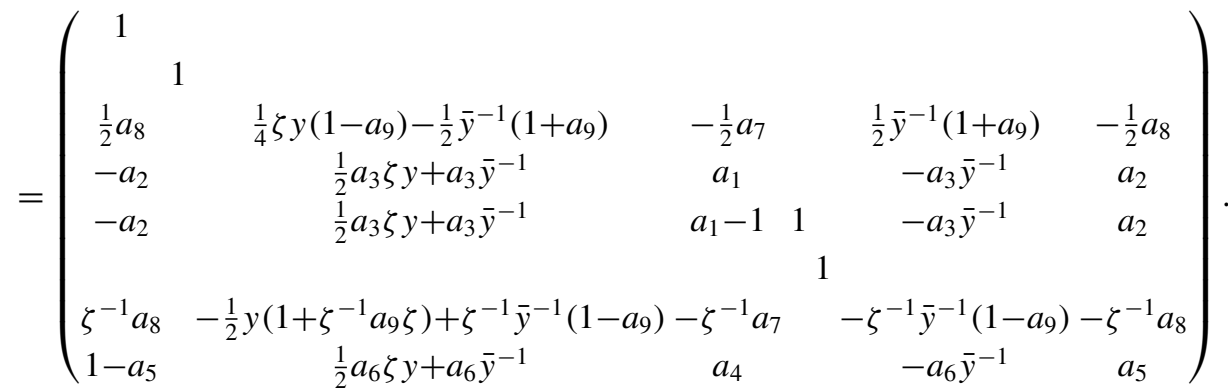

One checks the above matrix belongs to $Q_{x, y}$ if and only if the $a_{i}$ satisfy the conditions required by the definition of $\mathfrak{Y}$. The lemma follows by a similar argument to Lemma 4.38 below.

Definition 4.11. We will sometimes write $\mathfrak{Y}_{v}$ for the $\mathfrak{Y}$ above to emphasize the dependence on $v$. 
4C2. Fourier-Jacobi coefficient. We first give a formula for the Fourier coefficients for $\tilde{f}_{v, \text { sieg }}:=\rho\left(\tilde{\gamma}_{v}\right) f_{v, \text { sieg }}^{\dagger}$ and $\tilde{f}_{v, \text { sieg }}^{\prime}:=\rho\left(\tilde{\gamma}_{v}^{\prime}\right) f_{v, \text { sieg }}^{\dagger, \prime}$.

Lemma 4.12. (i) Let $\beta=\left(\beta_{i j}\right) \in S_{n+1}\left(F_{v}\right)$; then for all $z \in \mathbb{C}$ we have

$$
\begin{array}{r}
\tilde{f}_{v, \text { sieg, }, \beta}(z, 1)=\operatorname{Vol}\left(S_{n+1}\left(\bigcirc_{F, v}\right)\right) e_{v}\left(\operatorname{Tr}_{\mathscr{K}_{v} / F_{v}}\left(\frac{\beta_{a+b+2,1}+\cdots+\beta_{a+2 b+1, b}}{x}\right)\right. \\
\left.+\frac{\beta_{b+2, b+2}+\cdots+\beta_{b+1+a, b+1+a}}{y \bar{y}}\right) .
\end{array}
$$

(ii) Let $\beta=\left(\beta_{i j}\right) \in S_{v}\left(F_{v}\right)$. Then

$$
\begin{array}{r}
\tilde{f}_{v, \text { sieg, }, \beta}^{\prime}(z, 1)=\operatorname{Vol}\left(S_{n}\left(\mathscr{O}_{F, v}\right)\right) e_{v}\left(\operatorname{Tr}_{\mathscr{K}_{v} / F_{v}}\left(\frac{\beta_{a+b+1,1}+\cdots+\beta_{a+2 b, b}}{x}\right)\right. \\
\left.+\frac{\beta_{b+1, b+1}+\cdots+\beta_{b+a, b+a}}{y \bar{y}}\right) .
\end{array}
$$

The proof is straightforward.

Here we record a lemma on the Fourier-Jacobi coefficient for $f_{v}^{\dagger} \in I_{n}\left(\tau_{v}\right)$ and $\beta \in S_{m}\left(F_{v}\right)$.

Lemma 4.13. If $\beta \notin S_{m}\left(\widetilde{O}_{F_{v}}\right)^{*}$ then $\mathrm{FJ}_{\beta}\left(f^{\dagger} ; z, u, g, h y\right)=0$. If $\beta \in S_{n}\left(\bigcirc_{F_{v}}\right)^{*}$ then

$$
\mathrm{FJ}_{\beta}\left(f^{\dagger} ; z, u, g, h\right)=f^{\dagger}\left(z, g^{\prime} \eta\right) \omega_{\beta}\left(h, g^{\prime} \eta\right) \Phi_{0, y}(u) \cdot \operatorname{Vol}\left(S_{m}\left(\mathcal{O}_{F_{v}}\right)\right)
$$

where $g^{\prime}=\left(\begin{array}{ll}1_{n-m} & \\ & -1_{n-m}\end{array}\right) g\left(\begin{array}{ll}1_{n-m} & \\ & -1_{n-m}\end{array}\right)$.

The proof is similar to [Skinner and Urban 2014, Lemma 11.15].

4D. p-adic computations. In this subsection we first prove that, under some "generic conditions", the unique (up to scalar) nearly ordinary vector in $I(\rho)$ is just the unique (up to scalar) vector with certain prescribed action of level subgroup. Then we construct a section $F^{\dagger}$ in $I\left(\rho^{\vee}\right)$ which is the pullback of a Siegel section $f^{\dagger}$ supported in the big cell. We can understand the action of the level group of this section. Then we define $F^{0}$ to be the image of $F^{\dagger}$ under the intertwining operator. By checking the action of the level subgroup on $F^{0}$, we can prove that it is just the nearly ordinary vector.

In our calculations we will usually use the projection to the first component of $\mathscr{K}_{v} \simeq \mathscr{K}_{w} \times \mathcal{K}_{\bar{w}} \simeq \mathbb{Q}_{p} \times \mathbb{Q}_{p}$.

4D1. Nearly ordinary sections. Let $\lambda_{1}, \ldots, \lambda_{n}$ be $n$ characters of $F_{v}^{\times}$, which we identify with $\mathbb{Q}_{p}^{\times}$, and $\pi=\operatorname{Ind}_{B}^{\mathrm{GL}_{n}}\left(\lambda_{1}, \ldots, \lambda_{n}\right)$. 
Definition 4.14. Let $n=r+s$ and $\underline{k}=\left(c_{r+s}, \ldots, c_{s+1} ; c_{1}, \ldots, c_{s}\right)$ be a weight. We say $\left(\lambda_{1}, \ldots \lambda_{n}\right)$ is nearly ordinary with respect to $\underline{k}$ if

$$
\begin{aligned}
& \left\{\operatorname{val}_{p} \lambda_{1}(p), \ldots, \operatorname{val}_{p} \lambda_{n}(p)\right\}=\left\{c_{1}+s-1-\frac{1}{2} n+\frac{1}{2}, c_{2}+s-2-\frac{1}{2} n+\frac{1}{2}, \ldots, c_{s}-\frac{1}{2} n+\frac{1}{2},\right. \\
& \left.c_{s+1}+r+s-1-\frac{1}{2} n+\frac{1}{2}, \ldots, c_{r+s}+s-\frac{1}{2} n+\frac{1}{2}\right\} \text {. }
\end{aligned}
$$

We write the elements of the right side in order as $\kappa_{1}, \ldots, \kappa_{r+s}$, so $\kappa_{1}>\cdots>\kappa_{r+s}$. Let $\mathscr{A}_{p}:=\mathbb{Z}_{p}\left[t_{1}, t_{2}, \ldots, t_{n}, t_{n}^{-1}\right]$ be the Atkin-Lehner ring of $G\left(\mathbb{Q}_{p}\right)$, where $t_{i}$ is defined by $t_{i}=N\left(\mathbb{Z}_{p}\right) \alpha_{i} N\left(\mathbb{Z}_{p}\right), \alpha_{i}=\left(\begin{array}{ll}1_{n-i} & \\ & p 1_{i}\end{array}\right)$. Then $t_{i}$ acts on $\pi^{N\left(\mathbb{Z}_{p}\right)}$ by

$$
v \mid t_{i}=\sum_{x \in N \mid \alpha_{i}^{-1} N \alpha_{i}} x_{i} \alpha_{i}^{-1} v .
$$

We also define a normalized action with respect to the weight $\underline{k}$, following [Hida 2004b]:

$$
v \| t_{i}:=\delta\left(\alpha_{i}\right)^{-1 / 2} p^{\kappa_{1}+\cdots+\kappa_{i}} v \mid t_{i}
$$

Definition 4.15. A vector $v \in \pi$ is called nearly ordinary if it is an eigenvector for all $\| t_{i}$ with eigenvalues that are $p$-adic units.

We identify $\pi$ as a set of smooth functions on $\mathrm{GL}_{n}\left(\mathbb{Q}_{p}\right)$ :

$$
\pi=\left\{f: \mathrm{GL}_{n}\left(\mathbb{Q}_{p}\right) \rightarrow \mathbb{C} \mid f(b x)=\lambda(b) \delta_{B}(b)^{1 / 2} f(x)\right\} .
$$

Here, $\lambda(b):=\prod_{i=1}^{n} \lambda_{i}\left(b_{i}\right)$ for

$$
b=\left(\begin{array}{ccc}
b_{1} & \times & \times \\
& \ddots & \times \\
& & b_{n}
\end{array}\right)
$$

and $\delta_{B}$ is the modulus function for the upper-triangular Borel subgroup. Let $w_{\ell}$ be the longest Weyl element,

$$
\left(\begin{array}{llll} 
& & & \\
& & & \\
& & & \\
1 & &
\end{array}\right) \text {, }
$$

and let $f^{\ell}$ be the element in $\pi$ (which is unique up to scalar) that is supported in $B w_{\ell} N\left(\mathbb{Z}_{p}\right)$ and invariant under $N\left(\mathbb{Z}_{p}\right)$. We have:

Lemma 4.16. $f^{\ell}$ is an eigenvector for all $t_{i}$.

Proof. Note that, for any $i, f^{\ell} \mid t_{i}$ is invariant under $N\left(\mathbb{Z}_{p}\right)$. By looking at the definition of $v \mid t_{i}$ for the above model of $\pi$, it is not hard to see that $f^{\ell} \mid t_{i}$ is supported in $B\left(\mathbb{Q}_{p}\right) w_{\ell} B\left(\mathbb{Z}_{p}\right)$. So $f^{\ell} \mid t_{i}$ must be a multiple of $f^{\ell}$. 
Lemma 4.17. Suppose that $\left(\lambda_{1}, \ldots, \lambda_{n}\right)$ is nearly ordinary with respect to $\underline{k}$ and suppose

$$
v_{p}\left(\lambda_{1}(p)\right)>v_{p}\left(\lambda_{2}(p)\right)>\cdots>v_{p}\left(\lambda_{n}(p)\right)
$$

then the eigenvalues of $\| t_{i}$ acting on $f^{\ell}$ are $p$-adic units. In other words, $f^{\ell}$ is an ordinary vector.

Proof. A straightforward computation gives that

$$
f^{\ell} \| t_{i}=\lambda_{1} \cdots \lambda_{i}\left(p^{-1}\right) p^{\kappa_{1}+\cdots+\kappa_{i}} f^{\ell},
$$

which is clearly a $p$-adic unit by the definition of $\left(\lambda_{1}, \ldots, \lambda_{n}\right)$ being nearly ordinary with respect to $\underline{k}$.

Remark 4.18. Hida proved [2004b, Theorem 5.3] that the nearly ordinary vector is unique up to scalar.

Lemma 4.19. Let $\lambda_{1}, \ldots, \lambda_{a+2 b}$ be characters of $\mathbb{Q}_{p}^{\times}$such that $\operatorname{cond}\left(\lambda_{a+2 b}\right)>$ $\cdots>\operatorname{cond}\left(\lambda_{b+1}\right)>\operatorname{cond}\left(\lambda_{1}\right)>\cdots>\operatorname{cond}\left(\lambda_{b}\right)$. We define the subgroup $K_{\lambda}$ of $\mathrm{GL}_{a+2 b}\left(\mathbb{Z}_{p}\right)$ to be those matrices whose below-diagonal entries of the $i$-th column are divisible by cond $\left(\lambda_{a+2 b+1-i}\right)$ for $1 \leq i \leq a+b$, and the left-to-diagonal entries of the $j$-th row are divisible by cond $\left(\lambda_{a+2 b+1-j}\right)$ for $a+b+2 \leq j \leq a+2 b$. Let $\lambda^{\mathrm{op}}$ be the character of $K_{\lambda}$ defined by

$$
\lambda_{a+2 b}\left(g_{11}\right) \lambda_{a+2 b-1}\left(g_{22}\right) \cdots \lambda_{1}\left(g_{a+2 b} a+2 b\right) .
$$

Then $f^{\ell}$ is the unique (up to scalar) vector in $\pi$ such that the action of $K_{\lambda}$ is given by multiplying by $\lambda^{\text {op }}$.

Proof. We only need to prove the uniqueness. We use the model of induced representation as above. Let $n=a+2 b$ and let $e_{1}, \ldots, e_{n}$ be the standard basis of the standard representation of $\mathrm{GL}_{n}$. Let $p^{t_{i}}$ be the conductor of $\lambda_{i}$. So $t_{a+2 b}=\max \left\{t_{i}\right\}_{i}$. Write $K_{0}(p) \subset \mathrm{GL}_{n}\left(\mathbb{Z}_{p}\right)$ for the subgroup consisting of elements in $B\left(\mathbb{Z}_{p}\right)$ modulo $p$. Suppose $f$ is any vector satisfying the requirement of the lemma. Let $w$ be a Weyl element of $\mathrm{GL}_{n}$ such that $f$ is not identically 0 on $w K_{0}(p)$. Then we see that $w \cdot e_{1}=e_{a+2 b}$ by considering right-multiplication by $\operatorname{diag}\left(1+p^{t_{a+2 b}-1}, 1, \ldots, 1\right)$. Continuing this argument, we see that $w \cdot e_{2}=e_{a+2 b-1}, \ldots$ Finally, we have $w=w^{\ell}$ and the lemma is clear by Bruhat decomposition.

We let

$$
w_{1}:=\left(\begin{array}{ccccc}
1 & & & & \\
& \ddots & & & \\
& & 1 & & \\
& & & & \\
& & & & . \\
& & & 1
\end{array}\right) .
$$


Now let $\tilde{B}=B^{w_{1}}$ and $\tilde{K}_{\lambda}=K_{\lambda}^{w_{1}}$.

Corollary 4.20. Denote $a_{i}:=v_{p}\left(\lambda_{i}(p)\right)$. Suppose $\lambda_{1}, \ldots, \lambda_{a+2 b}$ are such that $\operatorname{cond}\left(\lambda_{1}\right)>\cdots>\operatorname{cond}\left(\lambda_{a+2 b}\right)$ and $a_{1}<\cdots<a_{a+b}<a_{a+2 b}<\cdots<a_{a+b+1}$. Then the unique (up to scalar) ordinary section with respect to $\tilde{B}$ is

$$
f^{\text {ord }}(x)= \begin{cases}\lambda_{1}\left(g_{11}\right) \cdots \lambda_{a+2 b}\left(g_{a+2 b, a+2 b}\right) & \text { if } g \in \tilde{K}_{\lambda}, \\ 0 & \text { otherwise. }\end{cases}
$$

Proof. We only need to prove that $\pi\left(w_{1}\right) f^{\text {ord }}(x)$ is ordinary with respect to $\tilde{B}^{w_{1}}=B$. Let $\lambda_{1}^{\prime}=\lambda_{a+b+1}, \ldots, \lambda_{b}^{\prime}=\lambda_{a+2 b}, \lambda_{b+1}^{\prime}=\lambda_{a+b}, \ldots, \lambda_{a+2 b}^{\prime}=\lambda_{1}$. Then $\lambda^{\prime}$ satisfies Lemma 4.17 and thus the ordinary section for $B$ (up to scalar) is $f_{\lambda^{\prime}}^{\ell}$. Since $\lambda^{\prime}$ also satisfies the assumptions of Lemma $4.19, f_{\lambda^{\prime}}^{\ell}$ is the unique section such that the action of $K_{\lambda}$ is given by $\lambda_{a+2 b}^{\prime}\left(g_{11}\right) \cdots \lambda_{1}^{\prime}\left(g_{a+2 b, a+2 b}\right)$. But $\lambda$ is clearly regular, so $\operatorname{Ind}_{B}^{\mathrm{GL}_{a+2 b}}(\lambda) \simeq \operatorname{Ind}_{B}^{\mathrm{GL}_{a+2 b}}\left(\lambda^{\prime}\right)$. So the ordinary section of $\operatorname{Ind}_{B}^{\mathrm{GL}_{a+2 b}}(\lambda)$ for $B$ also has the action of $K_{\lambda}$ given by this character. It is easy to check that $\pi\left(w_{1}\right) f^{\text {ord }}$ has this property and the uniqueness (up to scalar) gives the result.

4D2. Pullback sections. In this subsubsection we construct a Siegel section on $\mathrm{U}(a+2 b+1, a+2 b+1)$ which pulls back to the nearly ordinary Klingen sections on $\mathrm{U}(a+b+1, b+1)$. We need to rearrange the basis since we are going to study large block matrices and the new basis will simplify the explanation. One can check that the Klingen Eisenstein series we construct in this subsection, when going back to our previous basis, is nearly ordinary with respect to the Borel subgroup

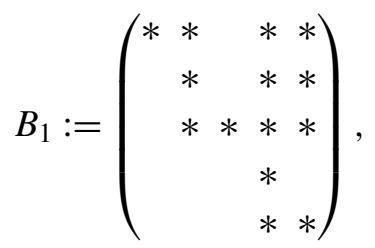

where the first four blocks are upper-triangular and the fifth is lower-triangular. But the one we need is nearly ordinary with respect to the Borel subgroup

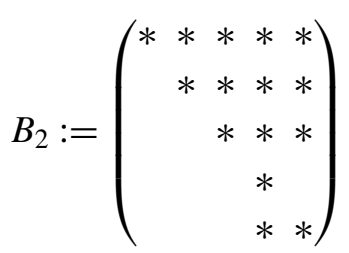

(it is for this one that we can use the $\Lambda$-adic Fourier-Jacobi expansions). (Here the blocks are with respect to the partition $b+1+a+b+1$.) There is a Weyl element $w_{\text {Borel }}$ of $\mathrm{GL}_{a+2 b+2}$ such that $w_{\text {Borel }}^{-1} B_{2} w_{\text {Borel }}=B_{1}$. This $w_{\text {Borel }}$ is in fact in the Weyl group of $\mathrm{GL}_{b+1+a}$ embedded as the upper-left minor. In the case of the doubling method $(\mathrm{U}(r, s) \times \mathrm{U}(s, r) \hookrightarrow \mathrm{U}(r+s, r+s))$ we have a corresponding change of 
index and we write $w_{\text {Borel }}^{\prime}$ for the corresponding Weyl element. In Section 4D4 we will come back to the original basis.

Now we explain the new basis. Let $V_{a, b}$ and $V_{a, b+1}$ be the Hermitian space with respective matrices

$$
\left(\begin{array}{lll}
\zeta 1_{a} & & \\
& & 1_{b} \\
& -1_{b}
\end{array}\right) \text { and }\left(\begin{array}{lll}
\zeta & & \\
& & 1_{b+1} \\
-1_{b+1} &
\end{array}\right) .
$$

(These are our skew-Hermitian spaces for $\mathrm{U}(r, s)$ and $\mathrm{U}(r+1, s+1)$ under the new basis.) The matrix $S$ for the embedding $\mathrm{U}\left(V_{a, b}\right) \times \mathrm{U}\left(V_{a, b+1}\right) \hookrightarrow \mathrm{U}\left(V_{a+2 b+1}\right)$ becomes

$$
\left(\begin{array}{ccccccccc}
1 & & & & & -\frac{1}{2} \zeta & & & \\
& & 1 & & & & & & \frac{1}{2} \\
& & 1 & & & & & \\
& & & -1 & & & \frac{1}{2} & & \\
-1 & & & & & -\frac{1}{2} \zeta & & & \\
& & & & 1 & & & & \\
& & & & & & & \\
& & & & & & & & \\
& -1 & & & & & & -\frac{1}{2}
\end{array}\right) .
$$

Godement sections at $p$. Let $v \mid p$ be a prime of $F$ and $\mathscr{K}_{v} \simeq \mathbb{Q}_{p} \times \mathbb{Q}_{p}$. Let $\tau$ be a character of $\mathbb{Q}_{p}^{\times} \times \mathbb{Q}_{p}^{\times}$. Suppose $\tau=\left(\tau_{1}, \tau_{2}^{-1}\right)$ and let $p^{s_{i}}$ be the conductor of $\tau_{i}, i=1,2$. Let $\chi_{1}, \ldots \chi_{a}, \chi_{a+1}, \ldots \chi_{a+2 b}$ be characters of $\mathbb{Q}_{p}^{\times}$whose conductors are $p^{t_{1}}, \ldots, p^{t_{a+2 b}}$. Suppose we are in the generic case:

Definition 4.21 (generic case).

$$
t_{1}>t_{2}>\cdots>t_{a+b}>s_{1}>t_{a+b+1}>\cdots>t_{a+2 b}>s_{2} .
$$

Also, let $\xi_{i}=\chi_{i} \tau_{1}^{-1}$ for $1 \leq i \leq a+b$ and $\xi_{j}=\chi_{j}^{-1} \tau_{2}$ for $a+b+2 \leq j \leq a+2 b+1$. Let $\xi_{a+b+1}=1$.

Let $\Phi_{1}$ be the following Schwartz function on $M_{a+2 b+1}\left(\mathbb{Q}_{p}\right)$ : let $\Gamma$ be the subgroup of $\mathrm{GL}_{a+2 b+1}\left(\mathbb{Z}_{p}\right)$ consisting of matrices $\gamma=\left(\gamma_{i j}\right)$ such that $p^{t_{k}}$ divides the below-diagonal entries (i.e., $i>j$ ) of the $k$-th column for $1 \leq k \leq a+b$ and $p^{s_{1}}$ divides $\gamma_{i j}$ when $a+b+2 \leq i \leq a+2 b+1$ and $j=a+b+1$; while $p^{t_{j-1}}$ divides $\gamma_{i j}$ when $a+b+2 \leq j \leq a+2 b+1$ and either $i \leq a+b+1$ or $i>j$.

Let $\xi_{i}^{\prime}=\chi_{i} \tau_{2}^{-1}, 1 \leq i \leq a+b, \xi_{j}^{\prime}=\chi_{j}^{-1} \tau_{1}, a+b+2 \leq j \leq a+2 b+1$, and $\xi_{a+b+1}^{\prime}=\tau_{1} \tau_{2}^{-1}$. (Thus, $\xi_{k}^{\prime}=\xi_{k} \tau_{1} \tau_{2}^{-1}$ for any $k$.)

Definition 4.22. $\Phi_{1}(x)= \begin{cases}0 & \text { if } x \notin \Gamma, \\ \prod_{k=1}^{a+b+1} \xi_{k}^{\prime}\left(x_{k k}\right) & \text { if } x \in \Gamma .\end{cases}$

Now we define another Schwartz function $\Phi_{2}$ on $M_{a+2 b+1}\left(\mathbb{Q}_{p}\right)$. 
Let $\mathfrak{X}$ be the following set: if

$$
x=\left(\begin{array}{llll}
A_{11} & A_{12} & A_{13} & A_{14} \\
A_{21} & A_{22} & A_{23} & A_{24} \\
A_{31} & A_{32} & A_{33} & A_{34} \\
A_{41} & A_{42} & A_{43} & A_{44}
\end{array}\right) \in \mathfrak{X}
$$

is in block matrix form with respect to the partition $a+2 b+1=a+b+1+b$, then

- $x$ has entries in $\mathbb{Z}_{p}$;

- $\left(\begin{array}{ll}A_{11} & A_{14} \\ A_{21} & A_{24}\end{array}\right)$ has $i$-th upper-left minors $A_{i}$ such that $\operatorname{det} A_{i} \in \mathbb{Z}_{p}^{\times}$for $i=1, \ldots, a+b$; and

- $A_{42}$ has $i$-th upper-left minors $B_{i}$ such that $\operatorname{det} B_{i} \in \mathbb{Z}_{p}^{\times}$for $i=1, \ldots, b$.

We define

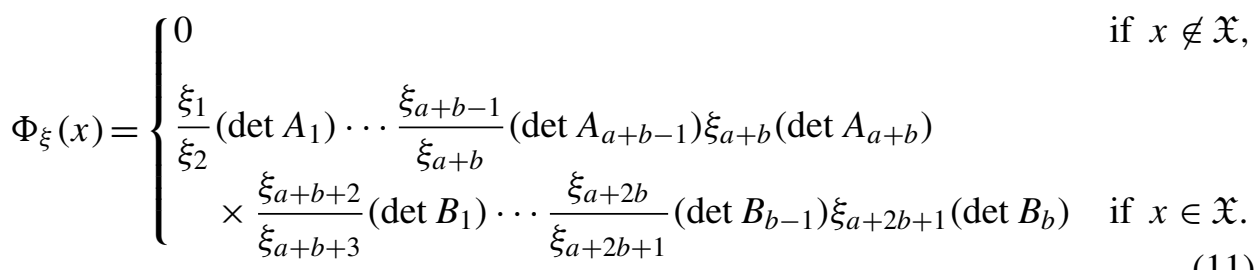

This is a locally constant function with compact support. Let

$$
\Phi_{2}(x):=\tilde{\Phi}_{\xi}(x)=\int_{M_{a+2 b+1}\left(\mathbb{Q}_{p}\right)} \Phi_{\xi}(y) e_{p}\left(\operatorname{Tr} y^{t} x\right) d y
$$

(where tilde stands for Fourier transform). Let $\Phi$ be the Schwartz function on $M_{a+2 b+1,2(a+2 b+1)}\left(\mathbb{Q}_{p}\right)$ defined by

$$
\Phi(X, Y):=\Phi_{1}(X) \Phi_{2}(Y)
$$

and define a Godement section (terminology of Jacquet) by

$f^{\Phi}(g)=\tau_{2}(\operatorname{det} g)|\operatorname{det} g|_{p}^{-s+(a+2 b+1) / 2}$

$$
\times \int_{\mathrm{GL}_{a+2 b+1}\left(\mathbb{Q}_{p}\right)} \Phi((0, X) g) \tau_{1}^{-1} \tau_{2}(\operatorname{det} X)|\operatorname{det} X|_{p}^{-2 s+a+2 b+1} d^{\times} X .
$$

Lemma 4.23. If $\gamma \in \Gamma$, then

$$
\Phi_{\xi}\left({ }^{t} \gamma X\right)=\prod_{k=1}^{a+2 b+1}\left(\xi_{k}\left(\gamma_{k k}\right)\right) \Phi_{\xi}(X) .
$$

Proof. This is straightforward. For example, to see that the $A_{42}$ block of ${ }^{t} \gamma X$ has invertible upper-left minors (i.e., has determinants in $\mathbb{Z}_{p}^{\times}$) for $\gamma \in \Gamma, X \in \mathfrak{X}$, one notes that all entries of the upper-right block of $\gamma$ are zero modulo $p$, and that 
multiplying by invertible matrices which are lower-triangular modulo $p$ does not change the property that all upper-left minors are invertible.

Fourier coefficients. For $z$ in the absolutely convergent range and $\beta \in S_{a+2 b+1}\left(\mathbb{Q}_{p}\right)$ (which is isomorphic to $M_{a+2 b+1}\left(\mathbb{Q}_{p}\right)$ through the first projection), the Fourier coefficient is defined by

$$
\begin{aligned}
& f_{\beta}^{\Phi}(1, z)=\int_{M_{a+2 b+1}\left(\mathbb{Q}_{p}\right)} f^{\Phi}\left(\left(\begin{array}{ll}
1_{a+2 b+1} & 1_{a+2 b+1}
\end{array}\right)\left(\begin{array}{cc}
1 & N \\
& 1
\end{array}\right)\right) e_{p}(-\operatorname{Tr} \beta N) d N \\
& =\int_{M_{a+2 b+1}\left(\mathbb{Q}_{p}\right)} \int_{\mathrm{GL}_{a+2 b+1}\left(\mathbb{Q}_{p}\right)} \Phi\left((0, X)\left(\begin{array}{rc} 
& 1_{a+2 b+1} \\
-1_{a+2 b+1} & -N
\end{array}\right)\right) \\
& \times \tau_{1}^{-1} \tau_{2}(\operatorname{det} X)|\operatorname{det} X|_{p}^{-2 z+a+2 b+1} e_{p}(-\operatorname{Tr} \beta N) d N d^{\times} X \\
& =\int_{\mathrm{GL}_{a+2 b+1}\left(\mathbb{Q}_{p}\right)} \Phi_{1}(-X) \Phi_{\xi}\left(-{ }^{t} X^{-1}{ }^{t} \beta\right) \tau_{1}^{-1} \tau_{2}(\operatorname{det} X)|\operatorname{det} X|_{p}^{-2 z} d^{\times} X \\
& =\tau_{1}^{-1} \tau_{2}(-1) \operatorname{Vol}(\Gamma) \Phi_{\xi}\left({ }^{t} \beta\right) \text {. }
\end{aligned}
$$

Definition 4.24. Let $\tilde{f}^{\dagger}=\tilde{f}_{a+2 b+1}^{\dagger}$ be the Siegel section supported on

$$
Q\left(\mathbb{Q}_{p}\right) w_{a+2 b+1}\left(\begin{array}{cc}
1 & M_{a+2 b+1}\left(\mathbb{Z}_{p}\right) \\
1
\end{array}\right)
$$

and $\tilde{f}^{\dagger}\left(w_{a+2 b+1}\left(\begin{array}{rr}1 & X \\ 1\end{array}\right)\right)=1$ for $X \in M_{a+2 b+1}\left(\mathbb{Z}_{p}\right)$.

$$
\text { Lemma 4.25. } \tilde{f}_{\beta}^{\dagger}(1)= \begin{cases}1 & \text { if } \beta \in M_{a+2 b+1}\left(\mathbb{Z}_{p}\right), \\ 0 & \text { if } \beta \notin M_{a+2 b+1}\left(\mathbb{Z}_{p}\right),\end{cases}
$$

(here we used the projection of $\beta$ onto its first component in $\mathscr{K}_{v}=F_{v} \times F_{v}$ ), where the first component corresponds to the element inside our CM-type $\Sigma_{\infty}$ under $\iota:=\mathbb{C} \simeq \mathbb{C}_{p}($ see Section $2 A)$.

Definition 4.26.

$$
f^{\dagger}:=\frac{f^{\Phi}}{\tau_{1}^{-1} \tau_{2}(-1) \operatorname{Vol}(\Gamma)} .
$$

Thus, $f_{\beta}^{\dagger}=\Phi_{\xi}\left({ }^{t} \beta\right)$.

We define

$$
c_{n}\left(\tau^{\prime}, z\right):= \begin{cases}\tau^{\prime}\left(p^{n t}\right) p^{2 n t z-t n(n+1) / 2} & \text { if } t>0, \\ p^{2 n z-n(n+1) / 2} & \text { if } t=0 .\end{cases}
$$

Now we recall a lemma from Skinner and Urban [2014, Lemma 11.12], which will be useful later.

Lemma 4.27. Suppose $v \mid p$ and $\beta \in S_{n}\left(\mathbb{Q}_{v}\right)$, $\operatorname{det} \beta \neq 0$. Then:

(i) If $\beta \notin S_{n}\left(\mathbb{Z}_{v}\right)$ then $M\left(z, \tilde{f}_{n}^{\dagger}\right)_{\beta}(-z, 1)=0$. 
(ii) Suppose $\beta \in S_{n}\left(\mathbb{Z}_{v}\right)$. Let $t:=\operatorname{ord}_{v}\left(\operatorname{cond}\left(\tau^{\prime}\right)\right)$. Then

$$
M\left(z, \tilde{f}_{n}^{\dagger}\right)_{\beta}(-z, 1)=\tau^{\prime}(\operatorname{det} \beta)|\operatorname{det} \beta|_{v}^{-2 z} \mathfrak{g}\left(\bar{\tau}^{\prime}\right)^{n} c_{n}\left(\tau^{\prime}, z\right) .
$$

Note that our $\tilde{f}^{\dagger}$ is the $f^{\dagger}$ in [Skinner and Urban 2014] and our $\tau$ is their $\chi$.

Now we want to write down our Godement section $f^{\Phi}$ in terms of $\tilde{f}^{\dagger}$. First we prove the following:

Lemma 4.28. Suppose $\Phi_{\xi, n}$ is the function on $M_{n}\left(\mathbb{Q}_{p}\right)$ defined as follows: If $\operatorname{cond}\left(\xi_{i}\right)=\left(p^{t_{i}}\right)$ for $t_{1}>\cdots>t_{n}$ and $\xi_{i}$ are characters of $\mathbb{Q}_{p}^{\times}$with conductor $p^{t_{i}}$. Let $\mathfrak{X}_{n}$ be the subset of $M_{n}\left(\mathbb{Z}_{p}\right)$ such that the $i$-th upper-left minor $M_{i}$ has determinant in $\mathbb{Z}_{p}^{\times}$. Define $\Phi_{\xi, n}$ to be

$$
\frac{\xi_{1}}{\xi_{2}}\left(\operatorname{det} M_{1}\right) \cdots \frac{\xi_{n-1}}{\xi_{n}}\left(\operatorname{det} M_{n-1}\right) \xi_{n}\left(\operatorname{det} M_{n}\right)
$$

on $\mathfrak{X}_{n}$ and 0 otherwise. Let

$$
\tilde{\mathfrak{X}}_{\xi, n}=\tilde{\mathfrak{X}}_{\xi}:=N\left(\mathbb{Z}_{p}\right)\left(\begin{array}{llll}
p^{-t_{1}} \mathbb{Z}_{p}^{\times} & & \\
& \ddots & \\
& & p^{-t_{n}} \mathbb{Z}_{p}^{\times}
\end{array}\right) N^{\mathrm{opp}}\left(\mathbb{Z}_{p}\right) .
$$

Then the Fourier transform $\hat{\Phi}_{\xi}$ of $\Phi_{\xi}$ is the function

$$
\begin{aligned}
\tilde{\Phi}_{\xi}(x)= \begin{cases}0 & \text { if } x \notin \tilde{\mathfrak{X}}_{\xi}, \\
\prod_{i=1}^{n} \mathfrak{g}\left(\xi_{i}\right) \prod_{i=1}^{n} \bar{\xi}_{i}\left(x_{i} p^{t_{i}}\right) & \text { if } x \in \tilde{\mathfrak{X}}_{\xi},\end{cases} \\
\text { where } x=\left(\begin{array}{lll}
1 & \times & \times \\
& \ddots & \times \\
& & 1
\end{array}\right)\left(\begin{array}{lll}
x_{1} & & \\
& \ddots & \\
& & x_{n}
\end{array}\right)\left(\begin{array}{lll}
1 & \\
\times & \ddots & \\
\times & \times & 1
\end{array}\right) .
\end{aligned}
$$

Proof. First suppose $x$ is in the "big cell" $N\left(\mathbb{Q}_{p}\right) T\left(\mathbb{Q}_{p}\right) N^{\mathrm{opp}}\left(\mathbb{Q}_{p}\right)$. It is easily seen that we can write $x$ in terms of block matrices,

$$
x=\left(\begin{array}{ll}
1_{n-1} & u \\
& 1
\end{array}\right)\left(\begin{array}{ll}
z & \\
& w
\end{array}\right)\left(\begin{array}{cc}
1_{n-1} & \\
v & 1
\end{array}\right),
$$

where $z \in \mathrm{GL}_{n-1}\left(\mathbb{Q}_{p}\right), w \in \mathbb{Q}_{p}^{\times}, u \in M_{n-1,1}\left(\mathbb{Q}_{p}\right)$ and $v \in M_{1, n-1}\left(\mathbb{Q}_{p}\right)$. A first observation is that $\tilde{\Phi}_{\xi}$ is invariant under right-multiplication by $N^{\mathrm{opp}}\left(\mathbb{Z}_{p}\right)$ and left-multiplication by $N\left(\mathbb{Z}_{p}\right)$. We show that $v \in M_{1 \times(n-1)}\left(\mathbb{Z}_{p}\right)$ if $\tilde{\Phi}_{\xi}(x) \neq 0$. By definition,

$$
\tilde{\Phi}_{\xi}(x)=\int_{M_{n}\left(Q_{p}\right)} \Phi_{\xi}(y) e_{p}\left(\operatorname{Tr} y^{t} x\right) d y,
$$

so, writing

$$
y=\left(\begin{array}{cc}
1_{n-1} & \\
\ell & 1
\end{array}\right)\left(\begin{array}{ll}
a & \\
& b
\end{array}\right)\left(\begin{array}{cc}
1 & m \\
& 1
\end{array}\right)=\left(\begin{array}{cc}
a & a m \\
\ell a & \ell a m+b
\end{array}\right),
$$


we have

$$
\begin{aligned}
& \tilde{\Phi}_{\xi}(x)=\int_{a \in \mathfrak{X}_{\xi, n-1}, m \in M\left(\mathbb{Z}_{p}\right), \ell \in M\left(\mathbb{Z}_{p}\right), b \in \mathbb{Z}_{p}^{\times}} \Phi_{\xi}\left(\left(\begin{array}{ll}
1 & \\
\ell & 1
\end{array}\right)\left(\begin{array}{ll}
a & \\
& b
\end{array}\right)\left(\begin{array}{cc}
1 & m \\
& 1
\end{array}\right)\right) \\
& \times e_{p}\left(\operatorname{Tr}\left(\left(\begin{array}{cc}
1 & \\
t_{m} & 1
\end{array}\right)\left(\begin{array}{ll}
{ }^{t} a & \\
& b
\end{array}\right)\left(\begin{array}{ll}
1 & { }^{t} \ell \\
& 1
\end{array}\right)\left(\begin{array}{ll}
1 & u \\
& 1
\end{array}\right)\left(\begin{array}{ll}
z & \\
& w
\end{array}\right)\left(\begin{array}{ll}
1 & \\
v & 1
\end{array}\right)\right)\right) d y \\
& =\int \Phi_{\xi}\left(\left(\begin{array}{ll}
a & \\
& b
\end{array}\right)\right) e_{p}\left(\operatorname{Tr}\left(\begin{array}{cc}
1 & \\
t_{m}+v & 1
\end{array}\right)\left(\begin{array}{cc}
{ }^{t} a & \\
& b
\end{array}\right)\left(\begin{array}{cc}
1 & { }^{t} \ell+u \\
& 1
\end{array}\right)\left(\begin{array}{ll}
z & \\
& w
\end{array}\right)\right) d y \\
& =\int \Phi_{\xi}\left(\left(\begin{array}{ll}
a & \\
& b
\end{array}\right)\right) \\
& \times e_{p}\left(\operatorname{Tr}\left(\left(\begin{array}{cc}
{ }^{t} a & { }^{t} a\left({ }^{t} \ell+u\right) \\
\left.\left({ }^{t} m+v\right)\right)^{t} a & \left({ }^{t} m+v\right){ }^{t} a\left({ }^{t} \ell+u\right)+b
\end{array}\right)\left(\begin{array}{ll}
z & \\
& w
\end{array}\right)\right)\right) d y \\
& =\int \Phi_{\xi}\left(\left(\begin{array}{ll}
a & \\
& b
\end{array}\right)\right) e_{p}\left(\operatorname{Tr}\left({ }^{t} a z+\left(\left({ }^{t} m+v\right)^{t} a\left({ }^{t} \ell+u\right)+b\right) w\right)\right) d y \text {. }
\end{aligned}
$$

(Note that $\Phi_{\xi}$ is invariant under transpose.)

If $\tilde{\Phi}_{\xi}(x) \neq 0$, then it follows from the last expression that $w \in p^{-t_{n}} \mathbb{Z}_{p}^{\times}$. Suppose $v \notin M_{1 \times(n-1)}\left(\mathbb{Z}_{p}\right) ;$ then ${ }^{t} m+v \notin M_{1 \times(n-1)}\left(\mathbb{Z}_{p}\right)$. We let $a, m$ and $b$ be fixed and let $\ell$ vary in $M_{1 \times(n-1)}\left(\mathbb{Z}_{p}\right)$; we find that this integral must be 0 . (Notice that $a \in \mathfrak{X}_{\xi, n-1}$ and $w \in p^{-t_{n}} \mathbb{Z}_{p}^{\times}$, thus $\left.\left({ }^{t} m+v\right)^{t} a w \notin M_{1 \times n-1}\left(\mathbb{Z}_{p}\right)\right)$. Thus, a contradiction. Therefore, $v \in M_{1 \times n-1}\left(\mathbb{Z}_{p}\right)$, and similarly $u \in M_{n-1,1}\left(\mathbb{Z}_{p}\right)$. Thus, by the observation at the beginning of the proof, we may assume $u=0$ and $v=0$ without loss of generality.

Thus, if we write $\Phi_{\xi, n-1}$ as the restriction of $\Phi_{\xi}$ to the upper-left $(n-1) \times(n-1)$ minor,

$$
\begin{aligned}
\tilde{\Phi}_{\xi}(x) & =\int \Phi_{\xi}\left(\left(\begin{array}{ll}
a & \\
& b
\end{array}\right)\right) e_{p}\left(\operatorname{Tr}\left({ }^{t} a z+\left({ }^{t} m^{t} a^{t} \ell+b\right) w\right)\right) d y \\
& =p^{-n t_{n}} \mathfrak{g}\left(\xi_{n}\right) \bar{\xi}_{n}\left(w p^{t_{n}}\right) \int_{a \in \mathfrak{X}_{\xi, n-1}} \Phi_{\xi, n-1}(a) e_{p}\left({ }^{t} a z\right) d y .
\end{aligned}
$$

By an induction procedure one gets

$$
\tilde{\Phi}_{\xi}(x)= \begin{cases}0 & \text { if } x \notin \tilde{\mathfrak{X}}_{\xi, n}, \\ p^{-\sum_{i=1}^{n} i t_{i}} \prod_{i=1}^{n} \mathfrak{g}\left(\xi_{i}\right) \prod_{i=1}^{n} \bar{\xi}_{i}\left(x_{i} p^{t_{i}}\right) & \text { if } x \in \tilde{\mathfrak{X}}_{\xi, n} .\end{cases}
$$

We have thus proved that $\tilde{\Phi}_{\xi, n}$, when restricted to the "big cell", has support in $\tilde{\mathfrak{X}}_{\xi, n}$. Since $\tilde{\mathfrak{X}}_{\xi, n}$ is compact, $\tilde{\Phi}_{\xi, n}$ itself must be supported in $\tilde{\mathfrak{X}}_{\xi, n}$. 
Lemma 4.29. Let $\tilde{\mathfrak{X}}_{\xi}$ be the support of $\Phi_{2}=\hat{\Phi}_{\xi}$; then a complete set of representatives of $\tilde{\mathfrak{X}}_{\xi} \bmod M_{a+2 b+1}\left(\mathbb{Z}_{p}\right)$ is given by the elements

$$
\left(\begin{array}{lll}
A & & B \\
C & & D \\
& & \\
& E &
\end{array}\right)
$$

where the blocks are with respect to the partition $a+b+1+b$ and $\left(\begin{array}{ll}A & B \\ C & D\end{array}\right)$ runs over the set

$$
\left(\begin{array}{cccc}
1 & m_{12} & \cdots & m_{1, a+b} \\
& \ddots & \ddots & \vdots \\
& & \ddots & m_{a+b-1, a+b} \\
& & & 1
\end{array}\right)\left(\begin{array}{cccc}
x_{1} & & & \\
& \ddots & & \\
& & \ddots & \\
& & & x_{a+b}
\end{array}\right)\left(\begin{array}{ccccc}
1 & & & \\
n_{21} & \ddots & & \\
\vdots & \ddots & \ddots & \\
n_{a+b, 1} & \cdots & n_{a+b, a+b-1} & 1
\end{array}\right),
$$

where $x_{i}$ runs over $p^{-t_{i}} \mathbb{Z}_{p}^{\times} \bmod \mathbb{Z}_{p}, m_{i j}$ runs over $\mathbb{Z}_{p} \bmod p^{t_{j}}, n_{i j}$ runs over $\mathbb{Z}_{p} \bmod p^{t_{i}}$, and $E$ runs over the set

$$
\left(\begin{array}{cccc}
1 & k_{12} & \cdots & k_{1, b} \\
& \ddots & \ddots & \vdots \\
& & \ddots & k_{b-1, b} \\
& & & 1
\end{array}\right)\left(\begin{array}{cccc}
y_{1} & & & \\
& \ddots & & \\
& & \ddots & \\
& & & y_{b}
\end{array}\right)\left(\begin{array}{cccc}
1 & & & \\
\ell_{21} & \ddots & \\
\vdots & \ddots & \ddots & \\
\ell_{b, 1} & \cdots & \ell_{b, b-1} & 1
\end{array}\right),
$$

where $y_{i}$ runs over $p^{-t_{i+a+b}} \mathbb{Z}_{p}^{\times} \bmod \mathbb{Z}_{p}, k_{i j}$ runs over $\mathbb{Z}_{p} \bmod p^{t_{a+b+j}}$, and $\ell_{i j}$ runs over $\mathbb{Z}_{p} \bmod p^{t_{a+b+i}}$.

Proof. This is elementary and we omit it here.

We also define, for $g \in \mathrm{GL}_{a+2 b}\left(\mathbb{Q}_{p}\right)$,

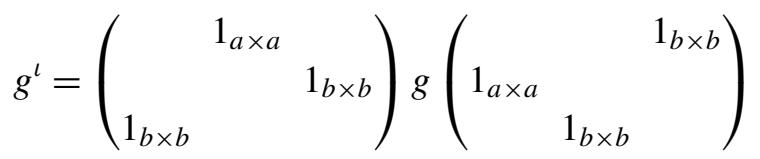

and

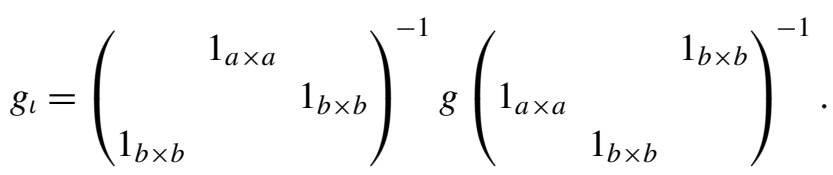


Corollary 4.30. We have

$$
\begin{aligned}
f^{\dagger}(z, g)= & p^{-\sum_{i=1}^{a+b} i t_{i}-\sum_{i=1}^{b} i t_{a+b+i}} \prod_{i=1}^{a+b} \mathfrak{g}\left(\xi_{i}\right) \xi_{i}(-1) \prod_{i=1}^{b} \mathfrak{g}\left(\xi_{a+b+1+i}\right) \xi_{a+b+1+i}(-1) \\
& \times \sum_{A, B, C, D, E} \prod_{i=1}^{a} \bar{\xi}_{i}\left(\frac{\operatorname{det} A_{i}}{\operatorname{det} A_{i-1}} p^{t_{i}}\right) \prod_{i=1}^{b} \bar{\xi}_{a+i, a+i}\left(\frac{\operatorname{det} D_{i}}{\operatorname{det} D_{i-1}} p^{t_{a+i}}\right) \\
& \times \prod_{i=1}^{b} \bar{\xi}_{a+b+1+i}\left(\frac{\operatorname{det} E_{i}}{\operatorname{det} E_{i-1}} p^{t_{a+b+i}}\right) \tilde{f}^{\dagger}\left(z, g\left(\begin{array}{rr}
A & B \\
1_{a+2 b+1} & D \\
& E \\
1_{a+2 b+1}
\end{array}\right)\right) .
\end{aligned}
$$

Here $A_{i}$ is the $i$-th upper-left minor of $A, D_{i}$ is the (a+i)-th upper-left minor of $\left(\begin{array}{ll}A & B \\ C & D\end{array}\right)$ (not of $\left.D\right), E_{i}$ is the $i$-th upper-left minor of $E$, and the sum runs over the set of representatives of Lemma 4.29.

Proof. We only need to check the Siegel Eisenstein sections on both sides coincide on $w N_{a+2 b+1}\left(\mathbb{Q}_{p}\right)$, since the big cell $Q_{a+2 b+1}\left(\mathbb{Q}_{p}\right) w N_{a+2 b+1}\left(\mathbb{Q}_{p}\right)$ is dense in $\mathrm{GL}_{2 a+4 b+2}$. To see this we just need to know that they have the same $\beta$-th Fourier coefficients for all $\beta \in S_{a+2 b+1}\left(\mathbb{Q}_{p}\right)$. But this is seen by (12) and Lemmas 4.28 and 4.29.

Now we define several sets. Let $\mathfrak{B}^{\prime}$ be the set of $(a+b) \times(a+b)$ upper-triangular matrices of the form

$$
\left(\begin{array}{cccc}
1 & m_{12} & \cdots & m_{1, a+b} \\
& \ddots & \ddots & \vdots \\
& & \ddots & m_{a+b-1, a+b} \\
& & & 1
\end{array}\right)\left(\begin{array}{cccc}
x_{1} & & & \\
& \ddots & & \\
& & \ddots & \\
& & & x_{a+b}
\end{array}\right),
$$

where $x_{i}$ runs over $\mathbb{Z}_{p}^{\times} \bmod p^{t_{i}}$ and $m_{i j}$ runs over $\mathbb{Z}_{p} \bmod p^{t_{j}}$.

Let $\mathfrak{D}^{\prime}$ be the set of $b \times b$ lower-triangular matrices of the form

$$
\left(\begin{array}{cccc}
1 & & \\
n_{21} & \ddots & \\
\vdots & \ddots & \ddots & \\
n_{a+b, 1} & \cdots & n_{a+b, a+b-1} & 1
\end{array}\right)
$$

where $n_{i j}$ runs over $\mathbb{Z}_{p} \bmod p^{t_{i+a+b}}$. 
Let $\mathfrak{E}^{\prime}$ be the set of $b \times b$ upper-triangular matrices of the form

$$
\left(\begin{array}{cccc}
1 & k_{12} & \cdots & k_{1, b} \\
& \ddots & \ddots & \vdots \\
& & \ddots & k_{b-1, b} \\
& & & 1
\end{array}\right)
$$

where $k_{i j}$ runs over $\mathbb{Z}_{p} \bmod p^{t_{a+b+j}}$.

Let $\mathfrak{C}^{\prime}$ be the set of $(a+b) \times(a+b)$ lower-triangular matrices of the form

$$
\left(\begin{array}{cccc}
y_{1} & & & \\
& \ddots & & \\
& & \ddots & \\
& & & y_{b}
\end{array}\right)\left(\begin{array}{cccc}
1 & & & \\
\ell_{21} & \ddots & & \\
\vdots & \ddots & \ddots & \\
\ell_{b, 1} & \cdots & \ell_{b, b-1} & 1
\end{array}\right),
$$

where $y_{i}$ runs over $\mathbb{Z}_{p}^{\times} \bmod p^{t_{i+a+b}} \mathbb{Z}_{p}$ and $\ell_{i j}$ runs over $\mathbb{Z}_{p} \bmod p^{t_{a+b+i}}$.

Thus, if $B^{\prime}, C^{\prime}, D^{\prime}, E^{\prime}$ run over the set $\mathfrak{B}^{\prime}, \mathfrak{C}^{\prime \prime}, \mathfrak{D}^{\prime}, \mathfrak{E}^{\prime}$, respectively, then

$$
\begin{aligned}
f^{\dagger}(z, g)=p^{-\sum_{i=1}^{a+b} i t_{i}-\sum_{i=1}^{b} i t_{a+b+i}} \\
\times \sum_{B^{\prime}, C^{\prime}, D^{\prime}, E^{\prime}} \prod_{i=1}^{a+b} \mathfrak{g}\left(\xi_{i}\right) \xi_{i}(-1) \prod_{i=1}^{b} \mathfrak{g}\left(\xi_{a+b+1+i}\right) \xi_{a+b+1+i}(-1) \\
\times \sum_{B^{\prime}, C^{\prime}, D^{\prime}, E^{\prime}} \prod_{i=1}^{a+b} \bar{\xi}_{i}\left(B_{i i}^{\prime}\right) \prod_{i=1}^{b} \xi_{a+b+i}\left(C_{i i}^{\prime}\right) \\
\times \tilde{f}^{\dagger}\left(z, g \alpha\left(\operatorname{diag}\left(B^{\prime}, 1, C^{\prime} 1\right),\left(\begin{array}{rr}
E^{\prime} & \\
D^{\prime}
\end{array}\right)^{\iota}\right)\left(\begin{array}{rr}
2 & A^{\prime} \\
1
\end{array}\right)\right. \\
\left.\times \alpha\left(\operatorname{diag}\left(B^{\prime}, 1, C^{\prime} 1\right),\left(\begin{array}{rr}
E^{\prime} & \\
& D^{\prime}
\end{array}\right)^{\iota}\right)^{-1}\right)
\end{aligned}
$$

$$
\begin{gathered}
=p^{-\sum_{i=1}^{a+b} i t_{i}-\sum_{i=1}^{b} i t_{a+b+1+i}} \\
\sum_{B^{\prime}, C^{\prime}, D^{\prime}, E^{\prime}} \prod_{i=1}^{a+b} \mathfrak{g}\left(\xi_{i}\right) \xi_{i}(-1) \prod_{i=1}^{b} \mathfrak{g}\left(\xi_{a+b+1+i}\right) \xi_{a+b+1+i}(-1) \\
\times \sum_{B^{\prime}, C^{\prime}, D^{\prime}, E^{\prime}} \prod_{i=1}^{a+b} \bar{\xi}_{i}\left(B_{i i}^{\prime}\right) \prod_{i=1}^{b} \xi_{a+b+i}\left(C_{i i}^{\prime}\right) \prod_{i=1}^{a+b} \bar{\tau}_{1}\left(B_{i i}^{\prime}\right) \prod_{i=1}^{b} \bar{\tau}_{2}\left(C_{i i}^{\prime}\right) \\
\times \tilde{f}^{\dagger}\left(z, g \alpha\left(\operatorname{diag}\left(B^{\prime}, 1, C^{\prime} 1\right),\left(\begin{array}{ll}
E^{\prime} & \\
& D^{\prime}
\end{array}\right)^{\iota}\right)\left(\begin{array}{rr}
1 & A^{\prime} \\
& 1
\end{array}\right)\right),
\end{gathered}
$$


where

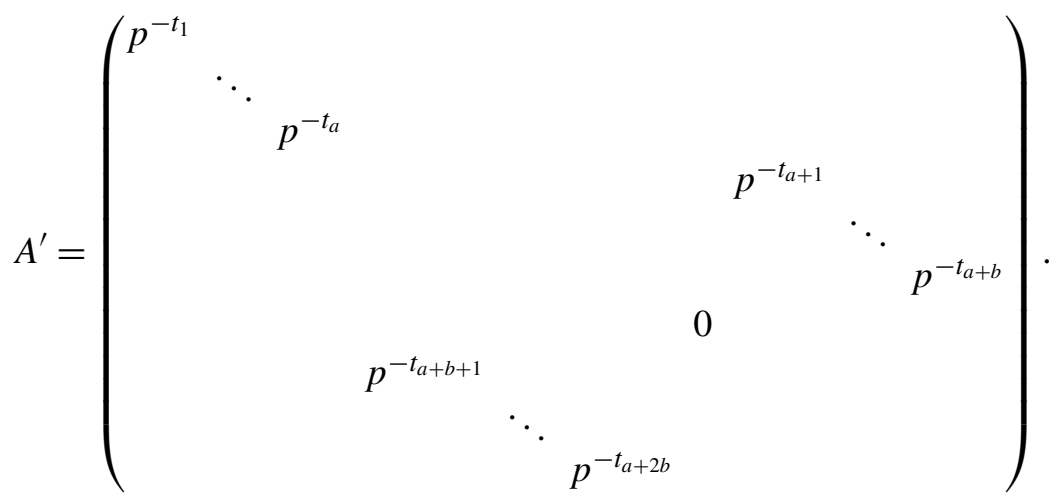

We let

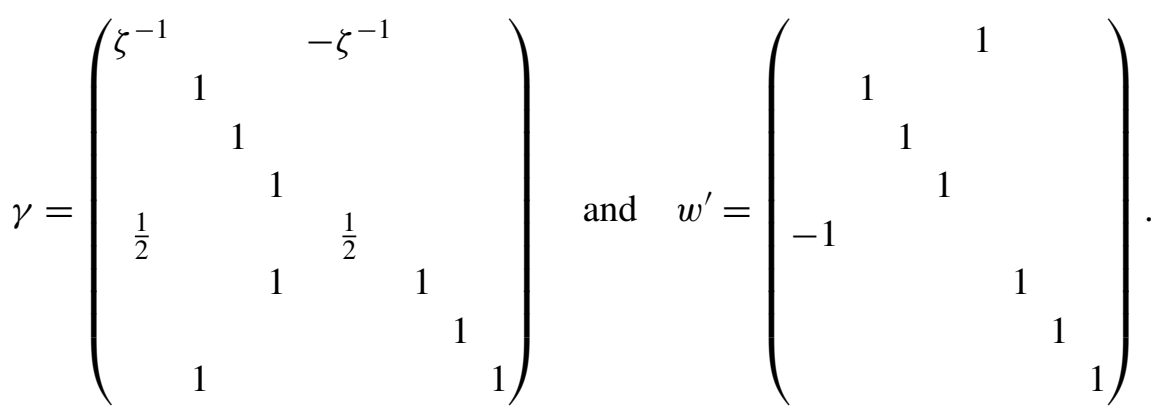

Definition 4.31 (pullback section). If $f$ is a Siegel section and $\varphi \in \pi_{p}$, then

$$
F_{\varphi}(z, f, g):=\int_{\mathrm{GL}_{a+2 b}\left(\mathbb{Q}_{p}\right)} f\left(z, \gamma \alpha\left(g, g_{1}\right) \gamma^{-1}\right) \bar{\tau}\left(\operatorname{det} g_{1}\right) \rho\left(g_{1}\right) \varphi d g_{1} .
$$

Now we define a subset $K$ of $\mathrm{GL}_{a+2 b+2}\left(\mathbb{Z}_{p}\right)$ so that $k \in K$ if and only if $p^{t_{i}}$ divides the below-diagonal entries of the $i$-th column for $1 \leq i \leq a+b, p^{s_{1}}$ divides the below-diagonal entries of the $(a+b+1)$-th column, and $p^{t_{a+b+j}}$ divides the rightto-diagonal entries of the $(a+b+1+j)$-th row for $1 \leq j \leq b-1$. We also define $v$, a character of $K$, by

$v(k)=\tau_{1}\left(k_{a+b+1, a+b+1}\right) \tau_{2}\left(k_{a+2 b+2, a+2 b+2}\right)$

$$
\times \prod_{i=1}^{a+b} \chi_{i}\left(k_{i i}\right) \prod_{i=1}^{b} \chi_{a+b+i}\left(k_{a+b+i+1, a+b+i+1}\right)
$$

for any $k \in K$.

Definition 4.32. We define $\Upsilon$ to be the element in $\mathrm{U}(n, n)\left(F_{v}\right)$ (which equals $\left.\mathrm{U}(n, n)\left(\mathbb{Q}_{p}\right)\right)$ such that the projection to the first component of $\mathscr{K}_{v}=F_{v} \times F_{v}$ equals that of $\gamma$ (note that $\gamma \notin \mathrm{U}(n, n)\left(F_{v}\right)$ ). 
Lemma 4.33. Let $K^{\prime} \subset K$ be the compact subgroup defined by, for $k \in K$,

$$
k=\left(\begin{array}{lllll}
a_{1} & a_{2} & a_{3} & b_{1} & b_{2} \\
a_{4} & a_{5} & a_{6} & b_{3} & b_{4} \\
a_{7} & a_{8} & a_{9} & b_{5} & b_{6} \\
c_{1} & c_{2} & c_{3} & d_{1} & d_{2} \\
c_{4} & c_{5} & c_{6} & d_{3} & d_{4}
\end{array}\right) \in K^{\prime}
$$

(here the blocks are with respect to the partition $(a+b+1+b+1))$ if and only if $p^{t_{a+b+i}+t_{j}}$ divides the $(i, j)$-th entry of $c_{1}$ for $1 \leq i \leq b, 1 \leq j \leq a$, and $p^{t_{a+b+i}+t_{a+j}}$ divides the $(i, j)$-th entry of $c_{2}$ for $1 \leq i \leq b, 1 \leq j \leq b$. (It is not hard to check that this is a group.)

Then $F_{\varphi}\left(z, \rho(\Upsilon) f^{\dagger}, g k\right)=v(k) F_{\varphi}\left(z, \rho(\Upsilon) f^{\dagger}, g\right)$ for any $\varphi \in \pi$ and $k \in K^{\prime}$. Proof. This follows directly from the action of $K^{\prime}$ on the Godement section $f^{\dagger}$.

We define $K^{\prime \prime}$ to be the subgroup of $K$ that consists of matrices

$$
\left(\begin{array}{ccccc}
1 & & & & \\
& 1 & & & \\
& & 1 & & \\
c_{1} & c_{2} & & 1 & \\
& & & & 1
\end{array}\right)
$$

such that $p^{t_{j}}$ divides the $(i, j)$-th entry of $c_{1}$ for $1 \leq i \leq b, 1 \leq j \leq a$, and $p^{t_{a+j}}$ divides the $(i, j)$-th entry of $c_{2}$ for $1 \leq i \leq b, 1 \leq j \leq b$.

Definition 4.34. Let $\tilde{K} \subset \mathrm{GL}_{a+2 b}\left(\mathbb{Z}_{p}\right)$ be the set of matrices

$$
\left(\begin{array}{lll}
a_{1} & a_{3} & a_{2} \\
a_{7} & a_{9} & a_{8} \\
a_{4} & a_{6} & a_{5}
\end{array}\right)
$$

(the blocks are with respect to the partition $(b+a+b))$ such that the columns of $a_{3}$ and $a_{6}$ are divisible by $p^{t_{1}}, \ldots, p^{t_{a}}$, the columns of $a_{4}$ are divisible by $p^{t_{a+1}}, \ldots, p^{t_{a+b}}, p^{t_{a+i}}$ divides the below-diagonal entries of the $i$-th column of $a_{1}$ $(1 \leq i \leq b), p^{t_{j}}$ divides the below-diagonal entries of the $j$-th column of $a_{9}$ $(1 \leq j \leq a)$, and $p^{t_{a+b+k}}$ divides the above-diagonal entries of the $k$-th row of $a_{5}$.

Let $\tilde{K}^{\prime} \subset \tilde{K}$ be those matrices such that $p^{t_{a+b+i}+t_{a+j}}$ divides the $(i, j)$-th entry of $a_{4}$ for $1 \leq i \leq b, 1 \leq j \leq b$, and $p^{t_{a+b+i}+t_{j}}$ divides the $(i, j)$-th entry of $a_{6}$ for $1 \leq i \leq b, 1 \leq j \leq a$. We also define $\tilde{K}^{\prime \prime}$ to be the subset of $\tilde{K}$ consisting of matrices

$$
\left(\begin{array}{ccc}
1 & & \\
& 1 & \\
a_{4} & a_{6} & 1
\end{array}\right)
$$


such that $p^{t_{a+j}}$ divides the $(i, j)$-th entry of $a_{4}$ for $1 \leq i \leq b, 1 \leq j \leq b$, and $p^{t_{j}}$ divides the $(i, j)$-th entry of $a_{6}$ for $1 \leq i \leq b, 1 \leq j \leq a$. We also define $\tilde{v}$, a character of $\tilde{K}$, by

$$
\tilde{v}(k)=\prod_{i=1}^{b} \chi_{a+i}\left(k_{i, i}\right) \prod_{i=1}^{a} \chi_{i}\left(k_{b+i, b+i}\right) \prod_{i=1}^{b} \chi_{a+b+i}\left(k_{a+b+i, a+b+i}\right) .
$$

The following lemma will be useful in identifying our pullback section:

Lemma 4.35. Suppose $F_{\varphi}\left(z, \rho(\Upsilon) f^{\dagger}, g\right)$ as a function of $g$ is supported in PwK and

$$
F_{\varphi}\left(z, \rho(\Upsilon) f^{\dagger}, g k\right)=v(k) F_{\varphi}\left(z, \rho(\Upsilon) f^{\dagger}, g\right)
$$

for $k \in K^{\prime}$, and $F_{\varphi}\left(z, \rho(\Upsilon) f^{\dagger}, w\right)$ is invariant under the action of $\left(\tilde{K}^{\prime \prime}\right)^{\iota}$. Then $F_{\varphi}\left(a, \rho(\Upsilon) f^{\dagger}, g\right)$ is the unique section (up to scalar) whose action by $k \in K$ is given by multiplying by $v(k)$.

Proof. This is easy from the fact that $K=K^{\prime} K^{\prime \prime}=K^{\prime \prime} K^{\prime}$. The uniqueness follows from Lemma 4.19.

From now on in this subsection we use $w$ to denote

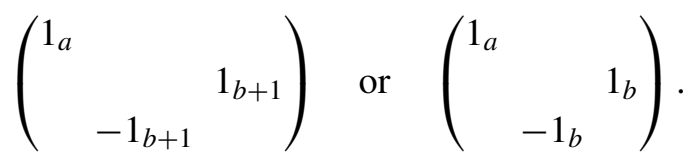

Lemma 4.36. If $\gamma \alpha(g, 1) \gamma^{-1} \in \operatorname{supp}\left(\rho(\Upsilon) f^{\dagger}\right)$ then $g \in P w K$. (Here $\rho$ denotes the action of $\mathrm{GU}_{a+2 b+1}\left(F_{v}\right)$ on the Siegel sections given by right-translation.)

Proof. Since $f^{\dagger}$ is of the form $f^{\dagger}(g)=\sum_{A \in \mathfrak{X}} \tilde{f}^{\dagger}\left(g\left(\begin{array}{cc}1 & A \\ 1\end{array}\right)\right)$, where $\mathfrak{X}$ is some set, we only have to check the lemma for each term in the summation.

Recall we defined $A^{\prime}$ in (14), where the blocks are with respect to the partition $(a+b+1+b)$. Let $\zeta_{v}$ and $\gamma_{v}$ be the projection of $\zeta$ and $\gamma_{v}$ to the first component of $\mathscr{K}_{v} \simeq F_{v} \times F_{v}$; then

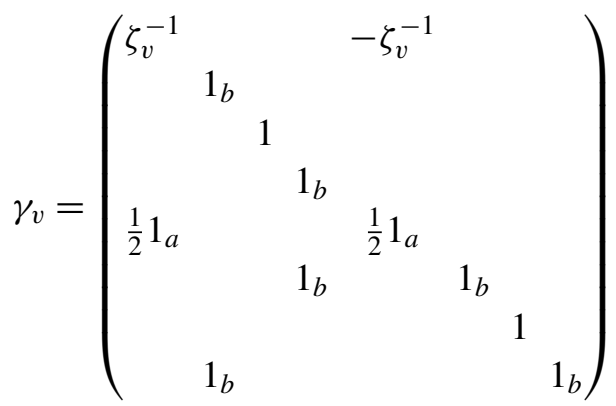




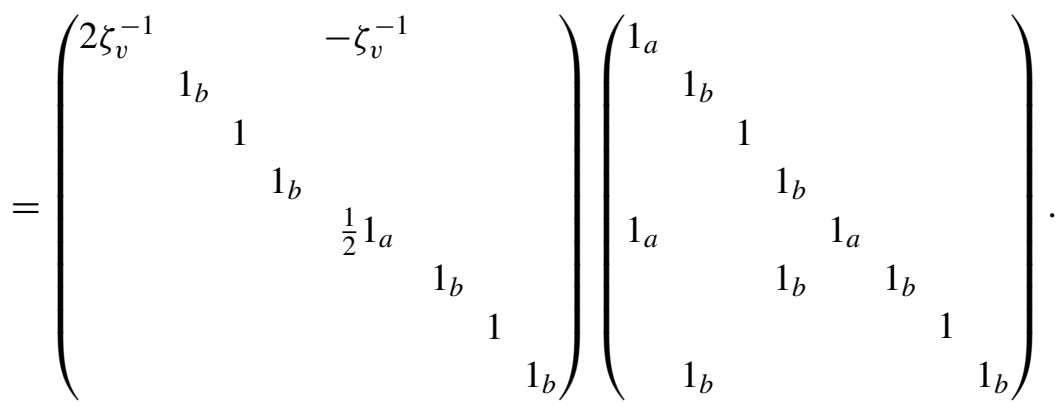

We denote the last term by $\tilde{\gamma}_{v}$ (different from the definition in the prime-to- $p$ case). Using the expression for $f^{\dagger}$ involving the various $B^{\prime}, C^{\prime}, D^{\prime}$ and $E^{\prime}$ as above and the fact that $\gamma(m(g, 1), g) \in Q$ and that $K$ is invariant under right-multiplication by any $B$ or $C$, we only need to check that if $\tilde{\gamma}_{v} \alpha(g, 1) \tilde{\gamma}_{v}^{-1} \in \operatorname{supp}\left(\rho(\Upsilon) \rho\left(\left(\begin{array}{cc}1 & A^{\prime} \\ 1\end{array}\right)\right)\right) \tilde{f}^{\dagger}$ then $g \in P w K$. Our calculations below are generalizations of the proof of [Skinner and Urban 2014, Proposition 11.16]. If

$$
g w=\left(\begin{array}{lllll}
a_{1} & a_{2} & a_{3} & b_{1} & b_{2} \\
a_{4} & a_{5} & a_{6} & b_{3} & b_{4} \\
a_{7} & a_{8} & a_{9} & b_{5} & b_{6} \\
c_{1} & c_{2} & c_{3} & d_{1} & d_{2} \\
c_{4} & c_{5} & c_{6} & d_{3} & d_{4}
\end{array}\right)
$$

then this is equivalent to

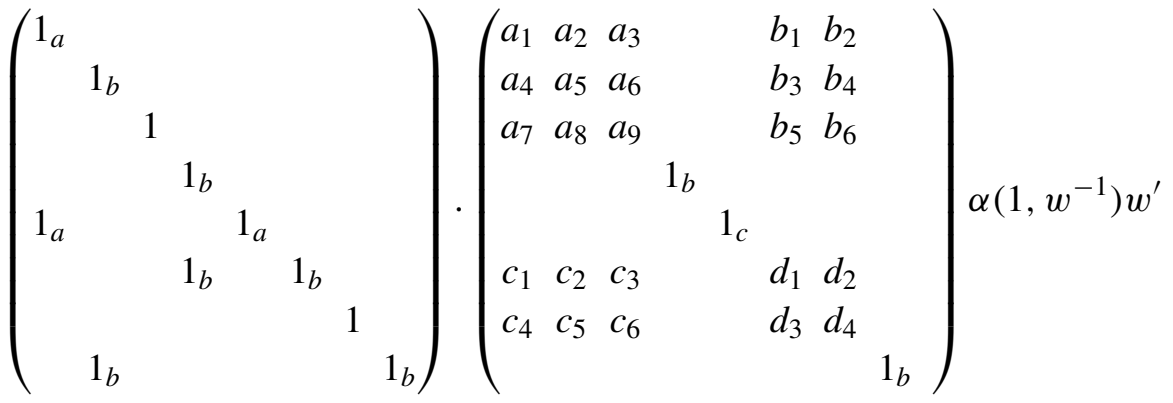

$$
\begin{aligned}
& \times \mathfrak{M}\left(\begin{array}{ccccccc}
1_{a} & & & & & & \\
& 1_{b} & & & & & \\
& & 1 & & & & \\
& & & 1_{b} & & & \\
-1_{a} & & & 1_{a} & & \\
& & -1_{b} & & 1_{b} & & \\
& & & & & 1 & \\
& -1_{b} & & & & 1_{b}
\end{array}\right) \mathfrak{M}^{-1} w_{a+2 b+1}^{-1}
\end{aligned}
$$


being in supp $\tilde{f}^{\dagger}$, where

$$
\mathfrak{M}=\operatorname{diag}\left(p^{t_{1}}, \ldots, p^{t_{a}}, 1_{b}, 1, p^{t_{a+1}}, \ldots, p^{t_{a+b}}, 1_{a}, 1_{b}, 1, p^{-t_{a+b+1}}, \ldots, p^{-t_{a+2 b}}\right)
$$

temporarily, which is equivalent to

$$
\tilde{\gamma}_{v} \alpha\left(g w,\left(\begin{array}{cc}
1 & 1_{a} \\
1_{b} &
\end{array}\right) \operatorname{diag}\left(p^{-t_{a+b+1}}, \ldots, p^{t_{1}}, \ldots, p^{t_{a+1}}, \ldots\right)\right) w^{\prime} \tilde{\gamma}_{v}^{-1}
$$

belonging to

$$
\operatorname{supp}\left(\rho\left(\mathfrak{M}^{-1} w_{a+2 b+1}\right) \tilde{f}^{\dagger}\right)
$$

The right-hand side is contained in

$$
Q_{t}:=Q \cdot\left\{\left(\begin{array}{ll}
1 \\
S & 1
\end{array}\right) \mid S \in S_{t}=\left(\begin{array}{llll}
S_{11} & S_{12} & S_{13} & S_{14} \\
S_{21} & S_{22} & S_{23} & S_{24} \\
S_{31} & S_{32} & S_{33} & S_{34} \\
S_{41} & S_{42} & S_{43} & S_{44}
\end{array}\right)\right\},
$$

where the blocks for $S_{t}$ are with respect to the partition $a+b+1+b$ and consist of matrices $S_{i j} \in M\left(\mathbb{Z}_{p}\right)$ such that $p^{t_{i}}$ divides the $i$-th column of the matrix $S$ for $1 \leq i \leq a, p^{t_{a+i}}$ divides the $(a+b+1+i)$-th column for $1 \leq i \leq b, p^{t_{a+b+i}}$ divides the $(a+b+1+i)$-th row for $1 \leq i \leq b$, and the $(i, j)$-th entry of $S_{41}$ and $S_{44}$ is divisible by $p^{t_{a+b+i}+t_{j}}$ and $p^{t_{a+b+i}+t_{a+j}}$, respectively. Observe that we have only to show that if $\tilde{\gamma} \alpha(g w, 1) w^{\prime} \tilde{\gamma}^{-1} \in Q_{t}$ then $g \in P w K$, i.e., $g w \in P K^{w}$ for $K^{w}:=w K w$ (note that $\left.\gamma\left(m\left(g_{1}, 1\right), g_{1}\right) \in Q\right)$.

Let

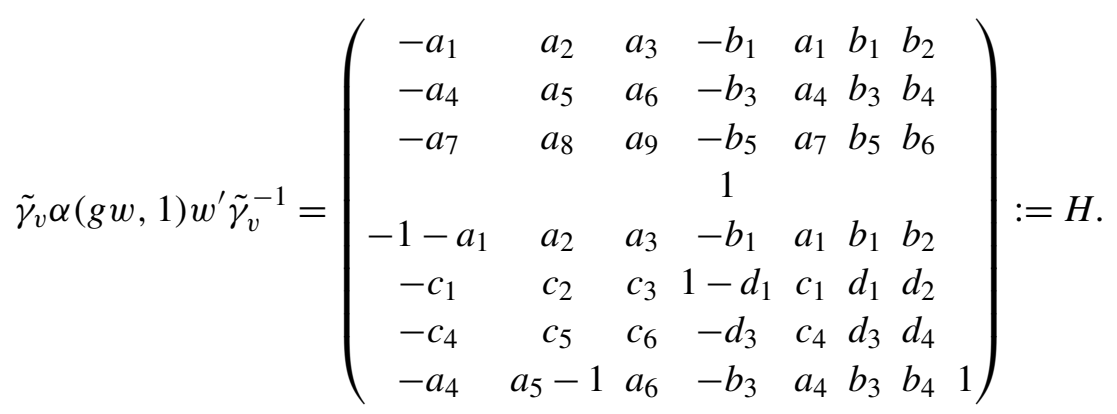

Thus, if $H \in Q_{t}$, then

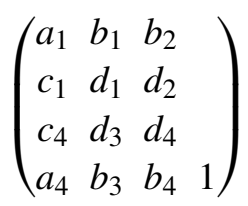


is invertible and there exists $S \in S_{t}$ such that

$$
\left(\begin{array}{cccc}
-1-a_{1} & a_{2} & a_{3} & -b_{1} \\
-c_{1} & c_{2} & c_{3} & 1-d_{1} \\
-c_{4} & c_{5} & c_{6} & -d_{3} \\
-a_{4} & a_{5}-1 & a_{6} & -b_{3}
\end{array}\right)=\left(\begin{array}{llll}
a_{1} & b_{1} & b_{2} \\
c_{1} & d_{1} & d_{2} \\
c_{4} & d_{3} & d_{4} \\
a_{4} & b_{3} & b_{4} & 1
\end{array}\right) S .
$$

By looking at the third row (blockwise), one finds $d_{4} \neq 0$, so by left-multiplying $g$ by a matrix

$$
\left(\begin{array}{ccccc}
1_{a} & & & & \times \\
& 1_{b} & & & \times \\
& & 1 & & \times \\
& & & 1_{b} & \times \\
& & & & d_{4}^{-1}
\end{array}\right)
$$

(which does not change the assumption and conclusion) we may assume that $d_{4}=1$ and $d_{2}=b_{2}=b_{4}=b_{6}=0$. So we assume that $g w$ is of the form

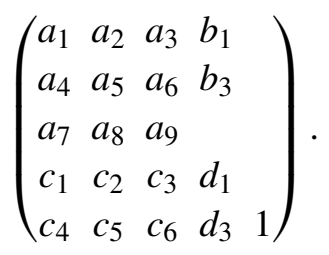

Next, by looking at the second row (blockwise) and noting that $d_{2}=0$, we find that $d_{1}$ is of the form

$$
\left(\begin{array}{ccccc}
\mathbb{Z}_{p}^{\times} & \mathbb{Z}_{p} & \cdots & \cdots & \mathbb{Z}_{p} \\
p^{t_{a+1}} \mathbb{Z}_{p} & \mathbb{Z}_{p}^{\times} & \ddots & \ddots & \mathbb{Z}_{p} \\
\vdots & p^{t_{a+2}} \mathbb{Z}_{p} & \mathbb{Z}_{p}^{\times} & \ddots & \vdots \\
\vdots & \ddots & \ddots & \ddots & \mathbb{Z}_{p} \\
p^{t_{a+1}} \mathbb{Z}_{p} & \cdots & \cdots & \cdots & \mathbb{Z}_{p}^{\times}
\end{array}\right) .
$$

So, by multiplying by a matrix of the form

$$
\left(\begin{array}{cccccc}
1_{a} & & & & \\
& 1_{b} & & & \\
& & 1 & \times & \\
& & & 1_{b} & \\
& & & &
\end{array}\right)
$$

on the left we may assume that $b_{5}=0$. Also, by looking at the third row again we see $c_{4}=\left(p^{t_{1}} \mathbb{Z}_{p}, \ldots, p^{t_{a}} \mathbb{Z}_{p}\right), c_{5}, c_{6} \in M\left(\mathbb{Z}_{p}\right)$ and $d_{3} \in\left(p^{t_{a+1}}, \ldots, p^{t_{a+b}}\right)$, while, from the second row, $c_{1} \in\left(M_{b \times 1}\left(p^{t_{1}} \mathbb{Z}_{p}\right), M_{b \times 1}\left(p^{t_{2}} \mathbb{Z}_{p}\right), \ldots, M_{b \times 1}\left(p^{t_{a}} \mathbb{Z}_{p}\right)\right)$, $c_{2} \in M_{b \times b}\left(\mathbb{Z}_{p}\right)$ and $c_{3} \in M_{b \times 1}\left(\mathbb{Z}_{p}\right)$. 
By looking at the first row and noting that $b_{2}=0$, we know

$$
a_{1} \in\left(\begin{array}{ccccc}
\mathbb{Z}_{p}^{\times} & \mathbb{Z}_{p} & \cdots & \cdots & \mathbb{Z}_{p} \\
p^{t_{1}} \mathbb{Z}_{p} & \mathbb{Z}_{p}^{\times} & \ddots & \ddots & \mathbb{Z}_{p} \\
\vdots & p^{t_{2}} \mathbb{Z}_{p} & \mathbb{Z}_{p}^{\times} & \ddots & \vdots \\
\vdots & \ddots & \ddots & \ddots & \mathbb{Z}_{p} \\
p^{t_{1}} \mathbb{Z}_{p} & \cdots & \cdots & \cdots & \mathbb{Z}_{p}^{\times}
\end{array}\right),
$$

$a_{2}, a_{3} \in M\left(\mathbb{Z}_{p}\right)$ and $b_{1} \in\left(M_{a \times 1}\left(p^{t_{a+1}} \mathbb{Z}_{p}\right), M_{a \times 1}\left(p^{t_{a+2}} \mathbb{Z}_{p}\right), \ldots, M_{a \times 1}\left(p^{t_{a+b}} \mathbb{Z}_{p}\right)\right)$. Finally, looking at the fourth row (blockwise), we note that $b_{4}=0$. Similarly,

$$
\begin{gathered}
a_{4} \in\left(M_{b \times 1}\left(p^{t_{1}} \mathbb{Z}_{p}\right), M_{b \times 1}\left(p^{t_{2}} \mathbb{Z}_{p}\right), \ldots, M_{b \times 1}\left(p^{t_{a}} \mathbb{Z}_{p}\right)\right), \\
b_{3} \in\left(M_{b \times 1}\left(p^{t_{a+1}} \mathbb{Z}_{p}\right), M_{b \times 1}\left(p^{t_{a+2}} \mathbb{Z}_{p}\right), \ldots, M_{b \times 1}\left(p^{t_{a+b}} \mathbb{Z}_{p}\right)\right), \\
a_{5}-1 \in\left(\begin{array}{c}
M_{1 \times b}\left(p^{t_{a+b+1}} \mathbb{Z}_{p}\right) \\
M_{1 \times b}\left(p^{t_{a+b+2}} \mathbb{Z}_{p}\right) \\
\vdots \\
M_{1 \times b}\left(p^{t_{a+2 b}} \mathbb{Z}_{p}\right)
\end{array}\right) \quad \text { and } \quad a_{6} \in\left(\begin{array}{c}
p^{t_{a+b+1}} \mathbb{Z}_{p} \\
p^{t_{a+b+2}} \mathbb{Z}_{p} \\
\vdots \\
p^{t_{a+2 b}} \mathbb{Z}_{p}
\end{array}\right) .
\end{gathered}
$$

Now we prove that $g w \in P K^{w}$ using the properties proven above. First we right-multiply $g w$ by

$$
\left(\begin{array}{ccccc}
1_{a} & & & \\
& 1_{b} & & & \\
& & 1 & & \\
-d_{1}^{-1} c_{1} & -d_{1}^{-1} c_{2} & -d_{1}^{-1} c_{3} & d_{1}^{-1} \\
-c_{4} & -c_{5} & -c_{6} & -d_{3} & 1
\end{array}\right) \in K^{w},
$$

which does not change the above properties or what needs to be proven, so without loss of generality we assume that $c_{4}=c_{5}=c_{6}=d_{3}=c_{1}=c_{2}=c_{3}=0$ and $d_{1}=1$. Moreover, we set $\left(\begin{array}{ll}a_{1} & a_{2} \\ a_{4} & a_{5}\end{array}\right)^{-1}\left(\begin{array}{l}a_{3} \\ a_{6}\end{array}\right):=T=\left(\begin{array}{l}T_{1} \\ T_{2}\end{array}\right)$. Then

$$
\left(\begin{array}{ccccc}
1_{a} & & T_{1} & & \\
& 1_{b} & T_{2} & & \\
& & 1 & & \\
& & & 1_{b} & \\
& & & & 1
\end{array}\right) \in K^{w}
$$

By multiplying

$$
\left(\begin{array}{ccccc}
1_{a} & & -T_{1} & & \\
& 1_{b} & & -T_{2} & \\
& & 1 & & \\
& & & 1_{b} & \\
& & & & 1
\end{array}\right)
$$


to the right we get an element in $P$. So it is clear that $g w \in P K^{w}$.

Now suppose that $\pi$ is nearly ordinary with respect to $\underline{k}$. We define $\varphi$ to be the unique (up to scalar) nearly ordinary vector in $\pi$ with respect to the Borel subgroup $\tilde{B}$. Let $\varphi_{w}=\pi(w) \varphi$.

Now write

$$
\varphi^{\prime}=\pi\left(\operatorname{diag}\left(p^{-t_{a+b+1}}, \ldots, p^{t_{1}}, \ldots, p^{t_{a+1}}, \ldots\right)^{\iota}\left(1_{1_{b}}{ }^{-1_{b}}\right)^{\iota}\right) \varphi_{w} .
$$

Compute the value $F_{\varphi^{\prime}}\left(z, \rho(\Upsilon) f^{\dagger}, w\right)$. In fact, $F_{\varphi^{\prime}}\left(z, \rho(\Upsilon) f^{\dagger}, w\right)$ is equal to

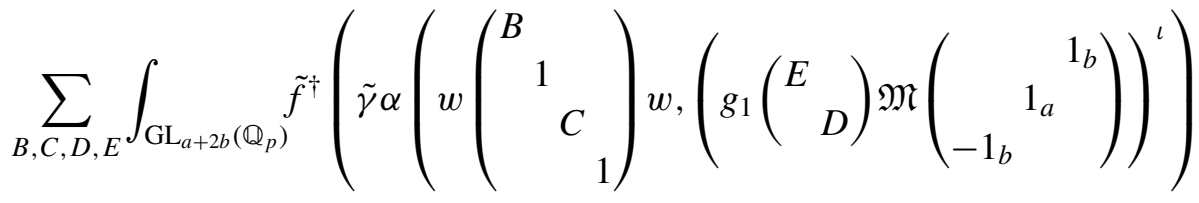

$$
\begin{aligned}
& \left.\times w^{\prime} \tilde{\gamma}^{-1} \Xi w_{a+2 b+1}^{-1}\right) \bar{\tau}\left(\operatorname{det} g_{1}\right) \rho\left(g_{1}^{l}\right) \varphi^{\prime} d g_{1}
\end{aligned}
$$

with (temporarily)

$$
\begin{aligned}
\mathfrak{M} & =\operatorname{diag}\left(p^{-t_{a+b+1}}, \ldots, p^{t_{1}}, \ldots, p^{t_{a+1}}, \ldots\right), \\
\Xi & =\operatorname{diag}\left(p^{-t_{1}}, \ldots, p^{-t_{a}}, 1_{b}, 1, p^{-t_{a+1}}, \ldots, p^{-t_{a+b}}, 1_{a}, 1_{b}, 1, p^{t_{a+b+1}}, p^{t_{a+2 b}}\right),
\end{aligned}
$$

where the sum is over $B \in \mathfrak{B}^{\prime}, C \in \mathfrak{C}^{\prime \prime}, D \in \mathfrak{D}^{\prime}$ and $E \in \mathfrak{E}^{\prime}$. A direct computation gives

$$
\tilde{\gamma} \alpha\left(1,\left(\begin{array}{lll}
a_{1} & a_{3} & a_{2} \\
a_{7} & a_{9} & a_{8} \\
a_{4} & a_{6} & a_{5}
\end{array}\right)^{\iota}\right) w^{\prime} \tilde{\gamma}^{-1}=\left(\begin{array}{cccccc}
-1_{a} & & \multicolumn{3}{c}{1_{a}} & \\
& 1_{b} & & & \\
& & 1 & & & \\
-a_{3} & -a_{2} & a_{1} & & a_{2} \\
-a_{9}-1_{a} & -a_{8} & a_{7} & 1_{a} & & a_{8} \\
-a_{3} & -a_{2} & a_{1}-1_{b} & 1_{b} & a_{2} \\
& & & & & 1 \\
-a_{6} & 1_{b}-a_{5} & a_{4} & & & a_{5}
\end{array}\right) .
$$

Now we define $\mathfrak{Y}$ to be the subset of $\mathrm{GL}_{a+2 b}\left(\mathbb{Z}_{p}\right)$ consisting of block matrices

$$
\left(\begin{array}{lll}
a_{1} & a_{3} & a_{2} \\
a_{7} & a_{9} & a_{8} \\
a_{4} & a_{6} & a_{5}
\end{array}\right)
$$


such that

$$
\tilde{\gamma} \alpha\left(1,\left(\begin{array}{ccc}
a_{1} & a_{3} & a_{2} \\
a_{7} & a_{9} & a_{8} \\
a_{4} & a_{6} & a_{5}
\end{array}\right)^{\iota}\right) w^{\prime} \tilde{\gamma}^{-1}
$$

is in the $Q_{t}$ defined in the proof of Lemma 4.36. It is not hard to prove that it can be described as follows: the $i$-th columns of $-a_{9}-1$ and $a_{3}$ (resp. $a_{7}$ and $a_{1}-1$ ) are divisible by $p^{t_{i}}$ (resp. $p^{t_{a+i}}$ ) for $1 \leq i \leq a$, the $(i, j)$-th entry of $a_{6}$ (resp. $a_{4}$ ) is divisible by $p^{t_{a+b+i}+t_{j}}$ (resp. $p^{t_{a+b+i}+t_{a+j}}$ ), and the $i$-th row of $1-a_{5}$ is divisible by $p^{t_{a+b+i}}$. The entries in $a_{2}$ and $a_{8}$ are in $\mathbb{Z}_{p}$. Then the pullback section is equal to

$$
\sum_{B, C, D, E} \int \tilde{f}^{\dagger}\left(\tilde{\gamma} \alpha\left(1, g_{1}^{\iota}\right) w^{\prime} \tilde{\gamma}^{-1} \Xi w_{a+2 b+1}^{-1}\right) \bar{\tau}\left(\operatorname{det} g_{1}\right) \pi\left(g_{1}^{\iota}\right) \varphi d g_{1},
$$

where

$$
\Xi=\operatorname{diag}\left(p^{-t_{1}}, \ldots, p^{-t_{a}}, 1,1, p^{-t_{a+1}}, \ldots, p^{-t_{a+b}}, 1,1,1, p^{t_{a+b+1}}, \ldots, p^{t_{a+2 b}}\right)
$$

and the integration is over elements (with superscript $w$ meaning conjugation by $w$ ) $g_{1} \in\left(\begin{array}{cc}B & \\ & C\end{array}\right)_{\iota}^{w} \mathfrak{Y}\left(\begin{array}{c}E \\ \\ \end{array}\right.$ for

$$
\begin{aligned}
\left(\begin{array}{ll}
E & \\
& D
\end{array}\right)_{\text {conj }}:=\left(\begin{array}{rl}
1_{b} \\
1_{a}
\end{array}\right) & \operatorname{diag}\left(p^{t_{a+b+1}}, \ldots, p^{-t_{1}}, \ldots, p^{-t_{a+1}}, \ldots\right)\left(\begin{array}{cc}
E & \\
D
\end{array}\right) \\
& \times \operatorname{diag}\left(p^{-t_{a+b+1}}, \ldots, p^{t_{1}}, \ldots, p^{t_{a+1}}, \ldots\right)\left(\begin{array}{c}
-1_{b} \\
1_{a} \\
1_{b}
\end{array}\right) .
\end{aligned}
$$

Lemma 4.37. If $\varphi_{w}$ is invariant under the action of $\left(\tilde{K}^{\prime \prime}\right)^{\iota}$, then

$$
F_{\varphi^{\prime}}\left(z, \rho(\Upsilon) f^{\dagger}, w\right)
$$

is such that the action of $\tilde{K}^{l}$ on it is given by $\tilde{v}$.

Proof. By the above two lemmas we only need to check that $F_{\varphi^{\prime}}\left(z, \rho(\Upsilon) f^{\dagger}, w\right)$ is invariant under the action of $\tilde{K}^{\prime \prime}$. We first claim that $\sum_{D, E} \pi\left(\left({ }^{E}{ }_{D}\right)_{\text {conj }}^{\iota}\right) \varphi^{\prime}$ is invariant under $\left(\tilde{K}^{\prime \prime}\right)^{\iota}$. The claim follows from direct checking. Also, for any $k_{1} \in \tilde{K}^{\prime \prime}$, we can find a $k_{2} \in \tilde{K}^{\prime \prime}$ such that $k_{1}\left({ }^{B}{ }_{C}\right)_{\iota}{ }_{\iota}^{w} k_{2}^{-1}$ runs over the same set of representatives as $\left({ }^{B}{ }_{C}\right)_{\iota}^{w}$. For any $k_{1} \in \tilde{K}^{\prime \prime}$, we can find a $k_{2} \in \tilde{K}^{\prime \prime}$ such that $k_{1} \mathfrak{Y} k_{2}^{-1}=\mathfrak{Y}$. The lemma follows from these observations. 
The value of $\tilde{f}^{\dagger}$ at

$$
g_{1}=\left(\begin{array}{c}
1_{b} \\
1_{a}
\end{array}\right) \operatorname{diag}\left(p^{t_{a+b+1}}, \ldots, p^{-t_{1}}, \ldots, p^{-t_{a+b}}, \ldots\right)
$$

is

$$
\tau\left(\left(p^{t_{a+b+1}+\cdots+t_{a+2 b}}, p^{t_{1}+\cdots+t_{a+b}}\right)\right)\left|p^{t_{1}+\cdots+t_{a+2 b}}\right|^{-z-(a+2 b+1) / 2} .
$$

So, a straightforward computation using the model for $\pi=\pi\left(\chi_{1}, \ldots, \chi_{a+2 b}\right)$ tells us the following:

Lemma 4.38. If $\varphi$ and $\varphi^{\prime}$ are defined as after the proof of Lemma 4.36, then:

$$
\begin{aligned}
& F_{\varphi^{\prime}}\left(z, \rho(\Upsilon) f^{\dagger}, w\right) \\
& =\tau\left(\left(p^{t_{1}+\cdots+t_{a+b}}, p^{t_{a+b+1}+\cdots+t_{a+2 b}}\right)\right)\left|p^{t_{1}+\cdots+t_{a+2 b}}\right|^{-z-(a+2 b+1) / 2} \operatorname{Vol}\left(\tilde{K}^{\prime}\right) \\
& \quad \times p^{-\sum_{i=1}^{a+b} i t_{i}-\sum_{i=1}^{b} i t_{a+b+i}} \prod_{i=1}^{a+b} \mathfrak{g}\left(\xi_{i}\right) \xi_{i}(-1) \prod_{i=1}^{b} \mathfrak{g}\left(\xi_{a+b+1+i}\right) \xi_{a+b+1+i}(-1) \varphi_{w} .
\end{aligned}
$$

Combining the three lemmas above, we get the following:

Proposition 4.39. With assumptions as in the above lemma, $F_{\varphi^{\prime}}\left(z, \rho(\Upsilon) f^{\dagger}, g\right)$ is the unique section supported in $P w K$ such that the right action of $K$ is given by multiplying the character $v$, and its value at $w$ is

$$
\begin{aligned}
& F_{\varphi^{\prime}}\left(z, \rho(\Upsilon) f^{\dagger}, w\right) \\
& =\tau\left(\left(p^{t_{1}+\cdots+t_{a+b}}, p^{t_{a+b+1}+\cdots+t_{a+2 b}}\right)\right)\left|p^{t_{1}+\cdots+t_{a+2 b}}\right|^{-z-\frac{a+2 b+1}{2}} \operatorname{Vol}\left(\tilde{K}^{\prime}\right) \\
& \quad \times p^{-\sum_{i=1}^{a+b} i t_{i}-\sum_{i=1}^{b} i t_{a+b+1+i}} \prod_{i=1}^{a+b} \mathfrak{g}\left(\xi_{i}\right) \xi_{i}(-1) \prod_{i=1}^{b} \mathfrak{g}\left(\xi_{a+b+1+i}\right) \xi_{a+b+1+i}(-1) \varphi_{w} .
\end{aligned}
$$

Proof. Clearly $\phi_{w}$ is invariant under $\left(\tilde{K}^{\prime \prime}\right)^{\iota}$.

This $F_{\varphi^{\prime}}\left(z, \rho(\Upsilon) f^{\dagger}, g\right)$ we constructed is not going to be the nearly ordinary vector unless we apply the intertwining operator to it. So now we start with some $\rho=(\pi, \tau)$. We define our Siegel section $f^{0} \in I_{a+2 b+1}(\tau)$ to be

$$
f^{0}(z ; g):=M\left(-z, f^{\dagger}\right)_{z}(g),
$$

where $f^{\dagger} \in I_{a+2 b+1}\left(\bar{\tau}^{c}\right)$. We recall the following generalization of a proposition from [Skinner and Urban 2014].

Proposition 4.40. Suppose our data $(\pi, \tau)$ comes from the local component at $v$ of a global data. Then there is a meromorphic function $\gamma^{(2)}(\rho, z)$ such that

$$
F_{\varphi \vee}(-z, M(z, f), g)=\gamma^{(2)}(\rho, z) A\left(\rho, z, F_{\varphi}(f ; z,-)\right)_{-z}(g) .
$$


Moreover, if $\pi_{v} \simeq \pi\left(\chi_{1}, \ldots, \chi_{a+2 b}\right)$ then, if we write $\gamma^{(1)}(\rho, z)=\gamma^{(2)}\left(\rho, z+\frac{1}{2}\right)$, then

$$
\gamma^{(1)}(\rho, z)=\psi(-1) c_{n}\left(\tau^{\prime}, z\right) \mathfrak{g}\left(\tau_{p}^{\prime}\right)^{n} \epsilon\left(\pi, \tau^{c}, z+\frac{1}{2}\right) \frac{L\left(\tilde{\pi}, \bar{\tau}^{c}, \frac{1}{2}-z\right)}{L\left(\pi, \tau^{c}, z+\frac{1}{2}\right)},
$$

where $c_{n}\left(\tau^{\prime}, z\right)$ is the constant appearing in Lemma 4.27.

Proof. The same as [Skinner and Urban 2014, Proposition 11.13].

Remark 4.41. Here we are using the $L$-factors for the base change from the unitary groups, while [Skinner and Urban 2014] uses the $\mathrm{GL}_{2} L$-factor for $\pi$, so our formula appears slightly different.

Now we are going to show that

$$
F_{v}^{0}(z ; g):=F_{\varphi^{\prime}}\left(z, \rho(\Upsilon) f^{0}, g\right)
$$

is a constant multiple of the nearly ordinary vector if our $\rho$ comes from the local component of the global Eisenstein data (see Section 3A). Return to the situation of our Eisenstein data. Suppose that at the Archimedean places our representation is a holomorphic discrete series associated to the (scalar) weight $\underline{k}=(0, \ldots 0 ; \kappa, \ldots \kappa)$ with $r$ zeroes and $s$ kappas. Here $r=a+b$ and $s=b$. Suppose $\pi \simeq \operatorname{Ind}\left(\chi_{1}, \ldots, \chi_{a+2 b}\right)$ is nearly ordinary with respect to the weight $\underline{k}$. We may reorder the $\chi_{i}$ so that $v_{p}\left(\chi_{1}(p)\right)=s-\frac{1}{2} n+\frac{1}{2}, \ldots, v_{p}\left(\chi_{r}(p)\right)=r+s-1-\frac{1}{2} n+\frac{1}{2}$, $v_{p}\left(\chi_{r+s}(p)\right)=\kappa-\frac{1}{2} n+\frac{1}{2}, \ldots, v_{p}\left(\chi_{r+1}(p)\right)=\kappa+s-1-\frac{1}{2} n+\frac{1}{2}$, and $\tau=\left(\tau_{1}, \tau_{2}^{-1}\right)$ is a character of $\mathbb{Q}_{p}^{\times} \times \mathbb{Q}_{p}^{\times}$with $v_{p}\left(\tau_{1}(p)\right)=v_{p}\left(\tau_{2}(p)\right)=\frac{\kappa}{2}$, so

$$
\begin{aligned}
v_{p}\left(\chi_{1}(p)\right)<\cdots<v_{p}\left(\chi_{a+b}(p)\right) & <v_{p}\left(\tau_{2}(p) p^{-z_{\kappa}}\right) \\
& <v_{p}\left(\tau_{1}(p) p^{z_{\kappa}}\right) \\
& <v_{p}\left(\chi_{a+2 b}(p)\right)<\cdots<v_{p}\left(\chi_{a+b+1}(p)\right),
\end{aligned}
$$

where $z_{\kappa}=\frac{1}{2}(\kappa-r-s-1)$. It is easy to see that

$$
I\left(\rho_{v}, z_{\kappa}\right) \simeq \operatorname{Ind}\left(\chi_{1}, \ldots \chi_{r+s}, \tau_{2}|\cdot|^{z_{\kappa}}, \tau_{1}|\cdot|^{-z_{\kappa}}\right) .
$$

By definition, $I\left(\rho_{v}, z_{\kappa}\right)$ is nearly ordinary with respect to the weight

$$
(\underbrace{0, \ldots, 0}_{r+1} ; \underbrace{\kappa, \ldots, \kappa}_{s+1}) .
$$

Definition 4.42. With assumptions and conventions as above, we say $(\pi, \tau)$ is generic if

$$
\begin{aligned}
\operatorname{cond}\left(\chi_{1}\right)>\cdots>\operatorname{cond}\left(\chi_{a+b}\right) & >\operatorname{cond}\left(\tau_{2}\right) \\
& >\operatorname{cond}\left(\chi_{a+b+1}\right)>\cdots>\operatorname{cond}\left(\chi_{a+2 b}\right)>\operatorname{cond}\left(\tau_{1}\right) .
\end{aligned}
$$


We suppose also that the conductor of $\tau_{i}$ is $p^{s_{i}}$. Notice that we have $s_{2}>s_{1}$ by our assumption, which is different from Definition 4.21 (since we have applied the intertwining operator here).

Let us record the following formula for the $\epsilon$-factor in Proposition 4.40:

$$
\begin{aligned}
\epsilon\left(\pi, \tau^{c}, z+\frac{1}{2}\right)= & \prod_{i=1}^{r} \mathfrak{g}\left(\chi_{i}^{-1} \tau_{2}\right) \chi_{i} \tau_{2}^{-1}\left(p^{t_{i}}\right) \cdot \prod_{i=1}^{s} \mathfrak{g}\left(\chi_{r+i}^{-1} \tau_{2}\right) \chi_{r+i} \tau_{2}^{-1}\left(p^{s_{2}}\right) \\
& \times\left|p^{\sum_{i=1}^{r} t_{i}+s \cdot s_{2}}\right|^{z+\frac{1}{2}} \prod_{i=1}^{r+s} \mathfrak{g}\left(\chi_{i} \tau_{1}^{-1}\right) \chi_{i}^{-1} \tau_{1}\left(p^{t_{i}}\right) \cdot\left|p^{\sum_{i=1}^{r+s} t_{i}}\right|^{z+\frac{1}{2}}
\end{aligned}
$$

From the form of $F_{\varphi^{\prime}}\left(z, \rho(\Upsilon) f^{\dagger} ; g\right)$ and the above proposition we have a description in the "generic" case for $F_{v}^{0}(z, g)$ as in [Skinner and Urban 2014, Lemma 9.6]: it is supported in $P\left(\mathbb{Q}_{p}\right) K_{v}$, with

$$
\begin{aligned}
F_{v}^{0}(z, 1)=\gamma^{(2)}(\rho,-z) \bar{\tau}^{c}\left(\left(p^{t_{1}+\cdots+t_{a+b}}, p^{t_{a+b+1}+\cdots+t_{a+2 b}}\right)\right) \\
\times\left|p^{t_{1}+\cdots+t_{a+2 b}}\right|^{z-(a+2 b+1) / 2} \operatorname{Vol}\left(\tilde{K}^{\prime}\right) p^{-\sum_{i=1}^{a+b}(i+1) t_{i}-\sum_{i=1}^{b}(i+1) t_{a+b+1+i}} \\
\times \prod_{i=1}^{a+b} \mathfrak{g}\left(\xi_{i}^{\dagger}\right) \xi_{i}(-1) \prod_{i=1}^{b} \mathfrak{g}\left(\xi_{a+b+1+i}^{\dagger}\right) \xi_{a+b+1+i}^{\dagger}(-1) \varphi \\
=c_{n}\left(\tau_{p}^{\prime},-z-\frac{1}{2}\right) \mathfrak{g}\left(\tau_{p}^{\prime}\right)^{n} \bar{\tau}^{c}\left(\left(p^{t_{1}+\cdots+t_{a+b}}, p^{t_{a+b+1}+\cdots+t_{a+2 b}}\right)\right) \\
\times\left|p^{t_{1}+\cdots+t_{a+2 b}}\right|^{z-(a+2 b+1) / 2} \operatorname{Vol}\left(\tilde{K}^{\prime}\right) p^{-\sum_{i=1}^{a+b} i t_{i}-\sum_{i=1}^{b} i t_{a+b+1+i}} \\
\quad \times \prod_{i=r+1}^{r+s} \mathfrak{g}\left(\chi_{i}^{-1} \tau_{2}\right) \chi_{i} \tau_{2}^{-1}\left(p^{s_{2}}\right) \prod_{j=1}^{r} \mathfrak{g}\left(\chi_{j} \tau_{1}^{-1}\right) \chi_{j}^{-1} \tau_{1}\left(p^{t_{j}}\right) \epsilon\left(\pi, \tau^{c}, z\right) \varphi \\
\quad \times\left|p^{\sum_{i=1}^{r} t_{i}+s \cdot s_{2}}\right|^{-z} \cdot\left|p^{\sum_{i=1}^{r+s} t_{i}}\right|^{-z},
\end{aligned}
$$

where the $\xi_{i}^{\dagger}$ are the $\xi_{i}$ defined in Definition 4.21 but using $\left(\pi, \bar{\tau}^{c}\right)$ instead of $(\pi, \tau)$. Here we also used Proposition 4.40 and the formula for the epsilon factor there. Notice that we have absorbed a factor $p^{-\sum_{i=1}^{a+b} t_{i}-\sum_{i=1}^{b} t_{a+b+1+i}}$, which comes from computing the image under the intertwining operator of $F_{\varphi^{\prime}}\left(z, \rho(\Upsilon) f^{\dagger} ; g\right)$ to get the factor $p^{-\sum_{i=1}^{a+b}(i+1) t_{i}-\sum_{i=1}^{b}(i+1) t_{a+b+1+i}}$ in the above expression. The right action of $K_{v}$ is given by the character

$$
\begin{aligned}
\chi_{1}\left(g_{11}\right) \cdots \chi_{a+b}\left(g_{a+b a+b}\right) \tau_{2}\left(g_{a+b+1}\right. & a+b+1) \chi_{a+b+1}\left(g_{a+b+2} a+b+2\right) \times \cdots \\
& \times \chi_{a+2 b}\left(g_{a+2 b+1} a+2 b+1\right) \tau_{1}\left(g_{a+2 b+2} a+2 b+2\right) .
\end{aligned}
$$

(It is easy to compute $A\left(\rho, z, F_{\varphi^{\prime}}\left(\rho(\Upsilon) f^{\dagger} ; z,-\right)\right)_{-z}(1)$ and we use the uniqueness of the vector with the required $K_{v}$ action. Here, on the second row of the above formula for $F_{v}^{0}(z, 1)$, the power for $p$ is slightly different from that for the section 
$F\left(z, f^{\dagger}, w\right)$. This comes from the computations for the intertwining operators for Klingen Eisenstein sections.)

Thus, Corollary 4.20 tells us that $F_{v}^{0}(z, g)$ is a nearly ordinary vector in $I(\rho)$.

Now we describe $f^{0}$ :

Definition 4.43. Suppose $\left(p^{t}\right)=\operatorname{cond}\left(\tau^{\prime}\right)$ for $t \geq 1$, then define $f_{t}$ to be the section supported in $Q\left(\mathbb{Q}_{p}\right) K_{Q}\left(p^{t}\right)$ with $f_{t}(k)=\tau\left(\operatorname{det} d_{k}\right)$ on $K_{Q}\left(p^{t}\right)$.

Lemma 4.44.

$$
\tilde{f}^{0}:=M\left(-z, \tilde{f}^{\dagger}\right)_{z}=f_{t, z} .
$$

Proof. This is just [Skinner and Urban 2014, Lemma 11.10].

Corollary 4.45. We have

$$
\begin{aligned}
f^{0}(z, g)=p^{-\sum_{i=1}^{a+b} i t_{i}-\sum_{i=1}^{b} i t_{a+b+i}} \prod_{i=1}^{a+b} \mathfrak{g}\left(\xi_{i}\right) \xi_{i}(-1) & \prod_{i=1}^{b} \mathfrak{g}\left(\xi_{a+b+1+i}\right) \xi_{a+b+1+i}(-1) \\
\times & \sum_{A, B, C, D, E} \prod_{i=1}^{a} \bar{\xi}_{i}\left(\frac{\operatorname{det} A_{i}}{\operatorname{det} A_{i-1}} p^{t_{i}}\right) \prod_{i=1}^{b} \bar{\xi}_{a+i, a+i}\left(\frac{\operatorname{det} D_{i}}{\operatorname{det} D_{i-1}} p^{t_{a+i}}\right) \\
& \times \prod_{i=1}^{b} \bar{\xi}_{a+b+1+i}\left(\frac{\operatorname{det} E_{i}}{\operatorname{det} E_{i-1}} p^{t_{a+b+i}}\right) \tilde{f}_{t}\left(z, g\left(\begin{array}{cc}
A & B \\
C & D \\
1_{a+2 b+1} & \\
& E \\
1_{a+2 b+1}
\end{array}\right)\right) .
\end{aligned}
$$

Here, $A_{i}$ is the $i$-th upper-left minor of $A, D_{i}$ is the (a+i)-th upper-left minor of $\left(\begin{array}{ll}A & B \\ C & D\end{array}\right)$ and $E_{i}$ is the $i$-th upper-left minor of $E$.

We define the Siegel section $f^{0 \prime} \in I_{a+2 b}(\tau)$ by

$$
\begin{aligned}
f^{0 \prime}(z, g)=p^{-\sum_{i=1}^{a+b} i t_{i}-\sum_{i=1}^{b} i t_{a+b+i}} \prod_{i=1}^{a+b} \mathfrak{g}\left(\xi_{i}\right) \xi_{i}(-1) \prod_{i=1}^{b} \mathfrak{g}\left(\xi_{a+b+1+i}\right) \xi_{a+b+1+i}(-1) \\
\times \sum_{A, B, C, D, E} \prod_{i=1}^{a} \bar{\xi}_{i}\left(\frac{\operatorname{det} A_{i}}{\operatorname{det} A_{i-1}} p^{t_{i}}\right) \prod_{i=1}^{b} \bar{\xi}_{a+i, a+i}\left(\frac{\operatorname{det} D_{i}}{\operatorname{det} D_{i-1}} p^{t_{a+i}}\right) \\
\times \prod_{i=1}^{b} \bar{\xi}_{a+b+1+i}\left(\frac{\operatorname{det} E_{i}}{\operatorname{det} E_{i-1}} p^{t_{a+b+i}}\right) \\
\\
\times \tilde{f}_{t}\left(\begin{array}{rrr}
z, g w_{\text {Borel }}^{\prime-1}\left(\begin{array}{rrr}
1_{a+2 b+1} & C & D \\
& E & B \\
1 & 1_{a+2 b+1}
\end{array}\right) w_{\text {Borel }}^{\prime}
\end{array}\right)
\end{aligned}
$$


Then, similar to before, the corresponding pullback section $F_{\varphi^{\prime}}^{\prime}\left(z, \rho\left(\Upsilon^{\prime}\right) f^{0 \prime}, 1\right)$ equals

$$
\begin{gathered}
c_{n}\left(\tau_{p}^{\prime},-z\right) \mathfrak{g}\left(\tau_{p}^{\prime}\right)^{n} \bar{\tau}^{c}\left(\left(p^{t_{1}+\cdots+t_{a+b}}, p^{t_{a+b+1}+\cdots+t_{a+2 b}}\right)\right)\left|p^{t_{1}+\cdots+t_{a+2 b}}\right|^{z-(a+2 b) / 2} \operatorname{Vol}\left(\tilde{K}^{\prime}\right) \\
\times p^{-\sum(i-1) t_{i}-\sum(i-1) t_{a+b+i}} \prod_{i=r+1}^{r+s} \mathfrak{g}\left(\chi_{i}^{-1} \tau_{2}\right) \chi_{i} \tau_{2}^{-1}\left(p^{s_{2}}\right) \\
\times \prod_{j=1}^{r} \mathfrak{g}\left(\chi_{j} \tau_{1}^{-1}\right) \chi_{j}^{-1} \tau_{1}\left(p^{t_{j}}\right) \epsilon\left(\pi, \tau^{c}, z+\frac{1}{2}\right) \varphi \\
\quad \times\left|p^{\sum_{i=1}^{r} t_{i}+s \cdot s_{2}}\right|^{-z+\frac{1}{2}} \cdot\left|p^{\sum_{i=1}^{r+s} t_{i}}\right|^{-z+\frac{1}{2}}
\end{gathered}
$$

Fourier coefficients for $f^{0}$. We record a formula here for the Fourier coefficients for $f^{0}$ which will be used in $p$-adic interpolation.

Lemma 4.46. Suppose $|\operatorname{det} \beta| \neq 0$; then:

(i) If $\beta \notin S_{a+2 b+1}\left(\mathbb{Z}_{p}\right)$ then $f_{\beta}^{0}(z, 1)=0$.

(ii) Let $t:=\operatorname{ord}_{p}\left(\operatorname{cond}\left(\tau^{\prime}\right)\right)$. If $\beta \in S_{a+2 b+1}\left(\mathbb{Z}_{p}\right)$ then

$$
f_{\beta}^{0}(z, 1)=\bar{\tau}^{\prime}(\operatorname{det} \beta)|\operatorname{det} \beta|_{p}^{2 z} \mathfrak{g}\left(\tau^{\prime}\right)^{a+2 b+1} c_{a+2 b+1}\left(\bar{\tau}^{\prime},-z\right) \Phi_{\xi}\left({ }^{t} \beta\right),
$$

where $c_{a+2 b+1}(-,-)$ is as defined in $(13)$ and $\Phi_{\xi}$ is defined in (11).

Proof. This follows from [Skinner and Urban 2014, Lemma 11.12] and the argument of Corollary 4.30, where we deduce the form of $f^{\dagger}$ from the section $\tilde{f}^{\dagger}$.

4D3. Fourier-Jacobi coefficients. Now let $m=b+1$. For $\beta \in S_{m}\left(F_{v}\right) \cap \mathrm{GL}_{m}\left(\mathcal{O}_{v}\right)$ we are going to compute the Fourier-Jacobi coefficient for $f_{t}$ at $\beta$.

Lemma 4.47. Let $x:=\left(\begin{array}{ll}1 & \\ D & 1\end{array}\right)$ (this is a block matrix with respect to $\left.(a+b)+(a+b)\right)$. Then:

(a) $\mathrm{FJ}_{\beta}\left(f_{t} ; z, v, x \eta^{-1}, 1\right)=0$ if $D \notin p^{t} M_{a+b}\left(\mathbb{Z}_{p}\right)$.

(b) If $D \in p^{t} M_{n}\left(\mathbb{Z}_{p}\right)$ then $\mathrm{FJ}_{\beta}\left(f_{t} ; z, v, x \eta^{-1}, 1\right)=c(\beta, \tau, z) \Phi_{0}(v)$, where

$$
c(\beta, \tau, z):=\bar{\tau}(-\operatorname{det} \beta)|\operatorname{det} \beta|_{v}^{2 z+n-m} \mathfrak{g}\left(\tau^{\prime}\right)^{m} c_{m}\left(\tau^{\prime},-z-\frac{1}{2}(n-m)\right)
$$

and $c_{m}$ is as defined in Lemma 4.27.

Proof. Similar to the proof of [Skinner and Urban 2014, Lemma 11.20]. We only give the detailed proof for the case when $a=0$. The case when $a>0$ is even easier to treat. 
Assuming $a=0$, we temporarily write $n$ for $b$ and save the letter $b$ for other use. We have

$$
w_{2 n+1}\left(\begin{array}{ccc}
1_{2 n+1} & S & v \\
& { }^{\bar{v}} & D \\
& 1_{2 n+1}
\end{array}\right) \alpha\left(1, \eta^{-1}\right)=\left(\begin{array}{cccc} 
& & 1_{n+1} & \\
& -1_{n} & & \\
-1_{n+1} & v & -S & \\
& D & -{ }^{t} \bar{v} & -1_{n}
\end{array}\right) .
$$

This belongs to $Q_{2 n+1}\left(\mathbb{Q}_{p}\right) K_{Q_{2 n+1}}\left(p^{t}\right)$ (where $K_{Q_{2 n+1}}\left(p^{t}\right)$ consists of matrices in $Q_{2 n+1}\left(\mathbb{Z}_{p}\right)$ modulo $\left.p^{t}\right)$ if and only if $S$ is invertible with $S^{-1} \in p^{t} M_{n+1}\left(\mathbb{O}_{v}\right)$, $S^{-1} v \in p^{t} M_{(n+1) \times n}\left(\mathcal{O}_{v}\right)$ and ${ }^{t} \bar{v} S^{-1} v-D \in p^{t} M_{n}\left(\mathbb{Z}_{p}\right)$. Since $v=\gamma^{t}(b, 0)$ for some $\gamma \in \mathrm{SL}_{n+1}\left(\mathbb{O}_{v}\right)$ and $b \in M_{n}\left(\mathscr{K}_{v}\right)$, we are reduced to the case $v={ }^{t}(b, 0)$. Writing $b=\left(b_{1}, b_{2}\right)$ with $b_{i} \in M_{n}\left(\mathbb{Q}_{p}\right)$, and $S=\left(T,{ }^{t} T\right)$ with $T \in M_{n+1}\left(\mathbb{Q}_{p}\right)$

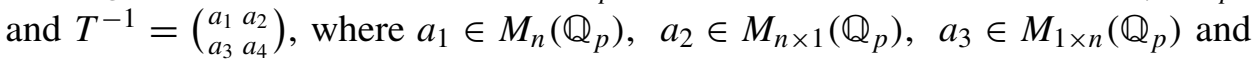
$a_{4} \in M_{1}\left(\mathbb{Q}_{p}\right)$, the conditions on $S$ and $v$ can be rewritten as

$$
\begin{gathered}
\operatorname{det} T \neq 0, \quad a_{i} \in p^{t} M_{n}\left(\mathbb{Z}_{p}\right), \quad a_{1} b_{1} \in p^{t} M_{n}\left(\mathbb{Z}_{p}\right), \quad a_{3} b_{1} \in p^{t} M_{1 \times n}\left(\mathbb{Z}_{p}\right), \\
{ }^{t} a_{1} b_{2} \in p^{t} M_{n}\left(\mathbb{Z}_{p}\right), \quad{ }^{t} a_{2} b_{2} \in p^{t} \mathbb{Z}_{p}, \quad{ }^{t} b_{2} a_{1} b_{1}-D \in p^{t} M_{n}\left(\mathbb{Z}_{p}\right) .
\end{gathered}
$$

Now we prove that if the integral for $\mathrm{FJ}_{\beta}\left(f_{t} ; z, v, x \eta^{-1}, 1\right)$ is nonzero then $b_{1}, b_{2} \in M_{n}\left(\mathbb{Z}_{p}\right)$. Suppose otherwise; then without loss of generality we assume $b_{1}$ has an entry which has the maximal $p$-adic absolute value among all entries of $b_{1}$ and $b_{2}$, Suppose it is $p^{w}$ for $w>0$ ( $w$ means this only inside this lemma). Also, for any matrix $A$ of given size, we say $A \in{ }^{t} b_{2}^{\vee}$ if and only ${ }^{t} b_{2} A$ has all entries in $\mathbb{Z}_{p}$ (of course we assume the sizes of the matrices are correct so that the product makes sense).

Now let

$$
\begin{array}{r}
\Gamma:=\left\{\gamma=\left(\begin{array}{ll}
h & j \\
k & l
\end{array}\right) \in \mathrm{GL}_{n}\left(\mathbb{Z}_{p}\right) \mid h \in \mathrm{GL}_{n+1}\left(\mathbb{Z}_{p}\right), l \in \mathbb{Z}_{p}^{\times}, h-1 \in{ }^{t} b_{2}^{\vee} \cap p^{t} M_{n}\left(\mathbb{Z}_{p}\right),\right. \\
\left.j \in \mathbb{Z}_{p}^{n} \cap{ }^{t} b_{2}^{\vee}, k \in p^{t} M_{1 \times n}\left(\mathbb{Z}_{p}\right)\right\} .
\end{array}
$$

Suppose that our $b_{1}, b_{2}$ and $D$ are such that there exist $a_{i}$ satisfying (*); then one can check that $\Gamma$ is a subgroup and, if $T$ satisfies (*), so does $T \gamma$ for any $\gamma \in \Gamma$. Let $\mathscr{T}$ denote the set of $T \in M_{n+1}\left(\mathbb{Q}_{p}\right)$ satisfying (*). Then

$$
\begin{aligned}
\mathrm{FJ}_{\beta}\left(f_{t} ; z, v,\left(\begin{array}{ll}
1 & \\
D & 1
\end{array}\right)\right. & \left.\eta^{-1}, 1\right) \\
& =\sum_{T \in \mathcal{T} / \Gamma}|\operatorname{det} T|_{p}^{3 n+2-2 z} \int_{\Gamma} \tau^{\prime}(-\operatorname{det} T \gamma) e_{p}(-\operatorname{Tr} \beta T \gamma) d \gamma .
\end{aligned}
$$

Let $T^{\prime}:=\beta T=\left(\begin{array}{ll}c_{1} & c_{2} \\ c_{3} & c_{4}\end{array}\right)$ (with blocks with respect to the partition $(n+1)$ ); then the above integral is zero unless we have $c_{1} \in p^{-t} M_{n}\left(\mathbb{Z}_{p}\right) \oplus\left[{ }^{t} b_{2}\right]_{n \times n}, \quad c_{4} \in p^{-t} \mathbb{Z}_{p}$, 
$c_{2} \in p^{-t} M_{n+1}\left(\mathbb{Z}_{p}\right)$ and $c_{3} \in\left[{ }^{t} b_{2}\right]_{1 \times n} \oplus M_{1 \times n}\left(\mathbb{Z}_{p}\right)$. Here $\left[{ }^{t} b_{2}\right]_{i \times n}$ means the set of $i \times n$ matrices such that each row is a $\mathbb{Z}_{p}$-linear combination of the rows of ${ }^{t} b_{2}$.

But then

$$
\beta\left(\begin{array}{c}
b_{1} \\
0
\end{array}\right)=T^{\prime} T^{-1}\left(\begin{array}{c}
b_{1} \\
0
\end{array}\right)=\left(\begin{array}{l}
c_{1} a_{1} b_{1}+c_{2} a_{3} b_{1} \\
c_{3} a_{1} b_{1}+c_{4} a_{3} b_{1}
\end{array}\right)
$$

Since $\beta \in \mathrm{GL}_{n+1}\left(\mathbb{Z}_{p}\right)$, the left-hand side must contain some entry with $p$-adic absolute value $p^{w}$. But it is not hard to see that all entries on the right-hand side have $p$-adic values strictly less than $p^{w}$; a contradiction. Thus we conclude that $b_{1} \in M_{n}\left(\mathbb{Z}_{p}\right)$ and $b_{2} \in M_{n}\left(\mathbb{Z}_{p}\right)$. By $\left({ }^{*}\right), b_{2}{ }^{t} a_{1} b_{1}-D \in p^{t} M_{n}\left(\mathbb{Z}_{p}\right)$ and $a_{1} \in p^{t} M_{n}\left(\mathbb{Z}_{p}\right)$. So $D \in p^{t} M_{n}\left(\mathbb{Z}_{p}\right)$.

The value claimed in part (b) can be deduced similarly to in [Skinner and Urban 2014, Lemma 11.20]

4D4. Original basis. Recall that we changed the basis at the beginning of this subsection. Now we go back. We define the corresponding sections (we use the same notations)

$$
\begin{aligned}
& f^{\dagger}(z, g)=p^{-\sum_{i=1}^{a+b} i t_{i}-\sum_{i=1}^{b} i t_{a+b+i}} \prod_{i=1}^{a+b} \mathfrak{g}\left(\xi_{i}\right) \xi_{i}(-1) \prod_{i=1}^{b} \mathfrak{g}\left(\xi_{a+b+1+i}\right) \xi_{a+b+1+i}(-1) \\
& \times \sum_{A, B, C, D, E} \prod_{i=1}^{a} \bar{\xi}_{i}\left(\frac{\operatorname{det} A_{i}}{\operatorname{det} A_{i-1}} p^{t_{i}}\right) \prod_{i=1}^{b} \bar{\xi}_{a+i, a+i}\left(\frac{\operatorname{det} D_{i}}{\operatorname{det} D_{i-1}} p^{t_{a+i}}\right) \\
& \times \prod_{i=1}^{b} \bar{\xi}_{a+b+1+i}\left(\frac{\operatorname{det} E_{i}}{\operatorname{det} E_{i-1}} p^{t_{a+b+i}}\right) \\
& \times \tilde{f}^{\dagger}\left(z, g w_{\text {Borel }}^{-1}\left(\begin{array}{cccccccc}
1_{b} & & & & & & C & D \\
& 1 & & & & & & \\
& & 1_{a} & & & & A & B \\
& & & 1_{b} & E & & & \\
& & & & 1_{b} & & & \\
& & & & 1 & & \\
& & & & & 1_{a} & \\
& & & & & & 1_{b}
\end{array}\right) w_{\text {Borel }}\right) \text {, }
\end{aligned}
$$

and $f^{0}(z, g)$ the same except using $\tilde{f}_{t}$ in place of $\tilde{f}^{\dagger}$. Here, $A_{i}$ is the $i$-th upper-left minor of $A, D_{i}$ is the $(a+i)$-th upper-left minor of $\left(\begin{array}{ll}A & B \\ C & D\end{array}\right)$ and $E_{i}$ is the $i$-th upperleft minor of $E$. The $w_{\text {Borel }}$ is the element in $G\left(F_{p}\right)$ such that, for any $v=w \bar{w}$ dividing $p$ with $w \in \Sigma_{p}$, its projection to the first factor of $\mathscr{K}_{v} \simeq \mathscr{K}_{w} \times \mathscr{K}_{\bar{w}}$ is the 
Weyl element defined at the beginning of Section 4D2. We also define

$$
\begin{aligned}
& f^{\dagger^{\prime}}(z, g)=p^{-\sum_{i=1}^{a+b} i t_{i}-\sum_{i=1}^{b} i t_{a+b+i}} \prod_{i=1}^{a+b} \mathfrak{g}\left(\xi_{i}\right) \xi_{i}(-1) \prod_{i=1}^{b} \mathfrak{g}\left(\xi_{a+b+1+i}\right) \xi_{a+b+1+i}(-1) \\
& \times \sum_{A, B, C, D, E} \prod_{i=1}^{a} \bar{\xi}_{i}\left(\frac{\operatorname{det} A_{i}}{\operatorname{det} A_{i-1}}\right) \prod_{i=1}^{b} \bar{\xi}_{a+i, a+i}\left(\frac{\operatorname{det} D_{i}}{\operatorname{det} D_{i-1}}\right) \\
& \times \prod_{i=1}^{b} \bar{\xi}_{a+b+1+i}\left(\frac{\operatorname{det} E_{i}}{\operatorname{det} E_{i-1}}\right) \\
& \times \tilde{f}^{\dagger}\left(z, g w_{\text {Borel }}^{\prime-1}\left(\begin{array}{cccccc}
1_{b} & & & & C & D \\
& 1_{a} & & & A & B \\
& & 1_{b} & E & & \\
& & & 1_{b} & & \\
& & & & 1_{a} & \\
& & & & & 1_{b}
\end{array}\right) w_{\text {Borel }}^{\prime}\right) \text {, }
\end{aligned}
$$

and $f^{0 \prime}$ the same except with $\tilde{f}_{t}$ instead of $\tilde{f}^{\dagger}$. The corresponding pullback section $F_{\varphi^{\prime}}\left(f^{0}, z,-\right)$ is the nearly ordinary section with respect to the Borel $B_{2}$ defined in Section $4 \mathrm{D} 2$ such that $F_{\varphi^{\prime}}\left(f^{0}, z, w_{\text {Borel }}\right)$ is given by

$$
\begin{aligned}
& c_{n+1}\left(\tau_{p}^{\prime},-z-\frac{1}{2}\right) \mathfrak{g}\left(\tau_{p}^{\prime}\right)^{n+1} \bar{\tau}^{c}\left(\left(p^{t_{1}+\cdots+t_{a+b}}, p^{t_{a+b+1}+\cdots+t_{a+2 b}}\right)\right) \\
& \times\left|p^{t_{1}+\cdots+t_{a+2 b}}\right|^{-z-(a+2 b+1) / 2} \operatorname{Vol}\left(\tilde{K}^{\prime}\right) p^{-\sum i t_{i}-\sum i t_{a+b+i}} \\
& \times \prod_{i=r+1}^{r+s} \mathfrak{g}\left(\chi_{i}^{-1} \tau_{2}\right) \chi_{i} \tau_{2}^{-1}\left(p^{t_{i}}\right) \prod_{j=1}^{r} \mathfrak{g}\left(\chi_{j} \tau_{1}^{-1}\right) \chi_{j}^{-1} \tau_{1}\left(p^{t_{j}}\right) \epsilon\left(\pi, \tau^{c}, z\right) \varphi .
\end{aligned}
$$

Also, we have that $F_{\varphi^{\prime}}^{\prime}\left(z, \rho\left(\Upsilon^{\prime}\right) f^{0^{\prime}}, w_{\text {Borel }}^{\prime}\right)$ is given by

$$
\begin{aligned}
c_{n}\left(\tau_{p}^{\prime},-z\right) \mathfrak{g}\left(\tau_{p}^{\prime}\right)^{n} \bar{\tau}^{c}\left(\left(p^{t_{1}+\cdots+t_{a+b}}, p^{t_{a+b+1}+\cdots+t_{a+2 b}}\right)\right)\left|p^{t_{1}+\cdots+t_{a+2 b}}\right|^{-z-(a+2 b) / 2} \operatorname{Vol}\left(\tilde{K}^{\prime}\right) \\
\times p^{-\sum i t_{i}-\sum i t_{a+b+i}} \prod_{i=r+1}^{r+s} \mathfrak{g}\left(\chi_{i}^{-1} \tau_{2}\right) \chi_{i} \tau_{2}^{-1}\left(p^{t_{i}}\right) \\
\times \prod_{j=1}^{r} \mathfrak{g}\left(\chi_{j} \tau_{1}^{-1}\right) \chi_{j}^{-1} \tau_{1}\left(p^{t_{j}}\right) \epsilon\left(\pi, \tau^{c}, z+\frac{1}{2}\right) \varphi .
\end{aligned}
$$

\section{Global computations}

\section{A. p-adic interpolation.}

5A1. Weight space and Eisenstein datum. Recall that we have the algebraic group $H=\prod_{v \mid p} \mathrm{GL}_{r} \times \mathrm{GL}_{s}$ such that $H\left(/ \mathbb{Z}_{p}\right)$ is the Galois group of the Igusa tower 
over the ordinary locus of the toroidal compactified Shimura variety. Let $T_{/ \mathbb{Z}_{p}}$ be the diagonal torus. Let $\boldsymbol{T}:=T\left(1+\mathbb{Z}_{p}\right)$. We define the weight ring $\Lambda=\Lambda_{r, s}$ as $\bigcirc_{L} \llbracket \boldsymbol{T} \rrbracket$. Fix throughout a finite-order character $\chi_{0}$ of $T\left(\mathbb{F}_{p}\right)$ (the torsion part of $T\left(\mathbb{Z}_{p}\right)$ ); a $\overline{\mathbb{Q}}_{p}$-point $\phi \in \operatorname{Spec} \Lambda$ is called arithmetic if there is a weight $\underline{k}=$ $\left(c_{s+1}, \ldots, c_{s+r} ; c_{1}, \ldots, c_{s}\right)=(0, \ldots, 0 ; \kappa, \ldots, \kappa)$ such that $\phi$ is given by a character $\chi_{0} \chi_{\phi} t_{1}^{c_{s+1}} \cdots t_{r}^{c_{r+s}} t_{r+1}^{-c_{1}} \cdots t_{r+s}^{-c_{s}}$ of $\boldsymbol{T}$ for $\chi_{\phi}$ a character of order and conductor powers of $p$, with $\kappa \geq 2(a+b+1)$. We write this $\kappa$ as $\kappa_{\phi}$. Let $\Lambda_{\mathscr{K}}=\mathscr{O}_{L} \llbracket \Gamma_{\mathscr{K}} \rrbracket$.

Definition 5.1. For $\llbracket$ a normal domain over $\Lambda$ which is also a finite module over $\Lambda$, a $\overline{\mathbb{Q}}_{p}$-point $\phi \in \operatorname{Spec} \llbracket$ is called arithmetic if its image in Spec $\Lambda$ is arithmetic.

(i) If $s>0$, let $V_{\infty, \infty}^{N}\left(K, \llbracket, \chi_{0}\right)$ be the set of घ-adic formal Fourier-Jacobi expansions

$$
\left\{\boldsymbol{f}_{x}=\sum_{\beta} a_{\beta}(x, \boldsymbol{f}) q^{\beta}\right\}_{x}
$$

such that, for a Zariski-dense set of generic arithmetic points $\phi \in \operatorname{Spec} \llbracket$, the specialization $\boldsymbol{f}_{\phi}$ is the formal Fourier-Jacobi expansion of a form on $\mathrm{U}(r, s)$ whose $p$-part nebentype at $\operatorname{diag}\left(t_{1}, \ldots, t_{r+s}\right)$ is given by

$$
\chi_{0} \chi_{\phi} \omega\left(t_{1}^{c_{s+1}} \cdots t_{r}^{c_{s+r}} t_{r+1}^{-c_{1}} \cdots t_{r+s}^{-c_{s}}\right)
$$

for the weight $\left(c_{s+1}, \ldots, c_{s+r} ; c_{1}, \ldots, c_{s}\right)=\left(0, \ldots, 0 ; \kappa_{\phi}, \ldots, \kappa_{\phi}\right)$. Here, by $\chi_{\phi}$ we also mean the character of $T\left(\mathbb{Z}_{p}\right)$ restricting to $\chi_{\phi}$ on $\boldsymbol{T}$ that is trivial on the torsion part of $T\left(\mathbb{Z}_{p}\right)$. We say $f \in V_{\infty, \infty}^{N}(K, \square)$ is a family of eigenforms if the specializations $f_{\phi}$ above are eigenforms. We define $V_{\infty, \infty}^{N \text {,ord }}\left(K, \mathbb{\square}, \chi_{0}\right)$ for the subspace such that the specializations above are nearly ordinary.

(ii) If $s=0$, then let $K=\prod_{v} K_{v}$ and let

$$
K_{0}(p)=\prod_{v \nmid p} K_{v} \prod_{v \mid p} K_{0}(p)_{v}
$$

(with $K_{0}(p)_{v} \subset G\left(\mathscr{O}_{F_{v}}\right)$ be the set of matrices which are in $B\left(\mathscr{O}_{F, v}\right)$ modulo $p$ ). Then $G(F) \backslash G\left(\mathbb{A}_{F}\right) / K_{0}(p)$ is a finite set with $\left\{g_{i}\right\}_{i}$ a set of representatives. We identify the set

$$
S_{G}^{N}(K):=G(F) \backslash G\left(\mathbb{A}_{F}\right) / K^{p} N\left(\mathscr{O}_{F, p}\right)
$$

with the disjoint union of $g_{i} \cdot N^{-}\left(p \hat{O}_{F, p}\right) T\left(\hat{O}_{F, p}\right)$ and endow the latter with the $p$-adic topology on $N^{-}\left(p \mathscr{O}_{F, p}\right) T\left(\mathscr{O}_{F, p}\right)$. We define $V_{\infty, \infty}^{N}\left(K, \mathbb{1}, \chi_{0}\right)$ to be the set of continuous $\llbracket$-valued functions on $S_{G}^{N}(K)$ such that, for a Zariski-dense set of arithmetic points $\phi \in \operatorname{Spec} \llbracket$, the specialization $f_{\phi}$ is a form on $\mathrm{U}(r, 0)$ whose $p$-part nebentype at $\operatorname{diag}\left(t_{1}, \ldots, t_{r}\right)$ is given by

$$
\chi_{0} \chi_{\phi} \omega\left(t_{1}^{c_{1}} \cdots t_{r}^{c_{r}}\right)
$$


for the weight $(0, \ldots, 0)$. Note that, by the description of nebentypus at $p$, such a family is determined by its values on $g_{i} \cdot N^{-}\left(p \mathscr{O}_{F, p}\right)$. Similarly we define $V_{\infty, \infty}^{N, \text { ord }}\left(K, \llbracket, \chi_{0}\right)$ for the nearly ordinary part.

Remark 5.2. To see this is a good definition, we have to compare it with the notion of Hida families in the literature. We refer to [Hida 2004b, Chapter 8; Hsieh 2014, Sections 3-4] for the definition of Hida families. We have to check that a Hida family in Hsieh's terms does give a Hida family here. We need to show that, if $\kappa_{\phi} \gg 0$ (depending on the $p$-part of the conductor at $\phi$ ) when $s>0$, then any nearly ordinary $p$-adic cusp form is classical. If $s>0$ this is proved by the argument of [Hsieh 2014, Theorem 4.19]. (It is assumed that $s=1$ in [loc. cit.]; however, the proof for this particular theorem works in the general case.) If $s=0$ the situation is even easier: the contraction property of the $U_{p}$ operator [Hsieh 2014, Proposition 4.4] (which again works in our case as well) shows that the specialization at $\phi$ is right-invariant under an open subgroup of $\mathrm{U}(r)\left(\mathbb{Z}_{p}\right)$ depending only on the conductor of the nebentypus (note also that we have trivial weight if $s=0$ ), and is thus classical.

Definition 5.3. We define an Eisenstein datum as a quadruple $\boldsymbol{D}:=\left(\llbracket, \boldsymbol{f}, \tau_{0}, \chi_{0}\right)$, where $\chi_{0}$ is a finite-order character of $T\left(\mathbb{Z}_{p}\right), \quad \tau_{0}$ is a finite-order character of $\mathscr{K}^{\times} \backslash \mathbb{A}_{\mathscr{K}}^{\times}$whose conductors at primes above $p$ divides $(p)$, and $\boldsymbol{f} \in V_{\infty, \infty}^{N \text {,ord }}(K, \square)$ is a Hida family of eigenforms defined as above. We define $\Lambda_{D}:=\Lambda \otimes_{O_{L}} \Lambda_{\mathscr{K}}$. We call a $\overline{\mathbb{Q}}_{p}$-point $\phi \in \operatorname{Spec} \Lambda_{D}$ is arithmetic if $\left.\phi\right|_{\square}$ is arithmetic with some weight $\kappa_{\phi}$ and $\phi\left(\gamma^{+}\right)=(1+p)^{\kappa_{\phi} / 2} \zeta_{+}, \phi\left(\gamma^{-}\right)=(1+p)^{\kappa_{\phi} / 2} \zeta_{-}$for $p$-power roots of unity $\zeta_{ \pm}$. We define $\tau_{\phi}=\phi \circ \Psi_{\mathscr{K}}$.

Let $\mathscr{X}$ be the set of arithmetic points. If $\boldsymbol{f}_{\phi}$ is classical and generates an irreducible automorphic representation $\pi_{f_{\phi}}$ of $\mathrm{U}(r, s)$, we say that $\phi$ is generic if $\left(\pi_{f_{\phi}}, \tau\right)$ is generic (see Definition 4.42). Let $\mathscr{Q}^{\text {gen }}$ be the set of generic arithmetic points.

\section{B. Some assumptions.}

5B1. Including types. Consider the group $\mathrm{U}(s, r)$. Suppose $K^{p}=K_{\Sigma} K^{\Sigma} \subset G\left(\mathbb{A}_{f}^{p}\right)$ for a finite set of primes $\Sigma$ and let $W_{\Sigma}$ be a finite $O_{L}$-module on which $K_{\Sigma}$ acts through a finite quotient. Let $K_{\Sigma}^{\prime} \subset K_{\Sigma}$ be a normal subgroup containing $\prod_{v \in \Sigma \backslash\{v \mid p\}} \mathfrak{Y}_{v}$, defined in Definition 4.11 and acting trivially on $W_{\Sigma}$, and let $K^{\prime}=G\left(\mathbb{Z}_{p}\right) K_{\Sigma}^{\prime} K^{\Sigma}$. The modules of modular forms of weight $\kappa$, type $W_{\Sigma}$ and character $\psi$ are

$$
M_{\kappa}\left(K, W_{\Sigma} ; \mathscr{O}_{L}\right)=\left(M_{\kappa}\left(K^{\prime} ; \mathscr{O}_{L}\right) \otimes_{\mathscr{O}_{L}} W_{\Sigma}\right)^{K_{\Sigma}} .
$$

Suppose for $v \in \Sigma \backslash\{v \mid p\}$ we have open compact subgroups $\tilde{K}_{v}^{\prime} \subset \tilde{K}_{v} \subset G\left(F_{v}\right)$ such that $\tilde{K}_{v}^{\prime}$ is a normal subgroup of $\tilde{K}_{v}$ and an irreducible finite-dimensional representation $W_{v}$ of $\tilde{K}_{v} / \tilde{K}_{v}^{\prime}$. Suppose $\varphi_{v} \in \pi_{v}$ is a vector in $W_{v}$. We fix a $\tilde{K}_{v}$-invariant 
measure and let $v_{1}, v_{2}, \ldots$ be a basis such that $\varphi_{v}$ is $v_{1}$. We also assume that $\tilde{K}_{v}^{\prime}$ includes the $\mathfrak{Y}_{v}^{\iota}$ defined in Section 4 . We let $W_{v}^{\vee}$ be the dual representation and we write $v_{1}^{\vee}, v_{2}^{\vee}, \ldots$ for the dual basis. We first prove the following lemma:

Lemma 5.4. Let $G$ be a finite group and $\rho: G \rightarrow \operatorname{Aut}(V)$ an irreducible representation on an $n$-dimensional vector space $V$. We fix a $G$-invariant norm and $a$ unitary basis $v_{1}, \ldots, v_{n}$. Let $\rho^{\vee}$ be the dual representation on $V^{\vee}$ with dual basis $v_{1}^{\vee}, \ldots, v_{n}^{\vee}$. Then, as elements in $V \otimes V^{\vee}$,

$$
\begin{aligned}
& \sum_{g \in G}\left(g v_{i} \otimes g v_{j}^{\vee}\right)=0, \quad i \neq j, \\
& \sum_{g}\left(g v_{i} \otimes g v_{i}^{\vee}\right)=|G| \sum_{i=1}^{n} v_{i} \otimes v_{i}^{\vee} .
\end{aligned}
$$

Proof. This is a straightforward application of the Schur orthogonal relation.

Definition 5.5. We define $W_{\Sigma \backslash\{p\}}=\prod_{v \in \Sigma \backslash\{p\}} W_{v}$ and $v_{1}=\prod_{v \in \Sigma \backslash\{p\}} v_{v, 1} \in W_{\Sigma \backslash\{p\}}$.

We can also make a notion of $W_{\Sigma \backslash\{p\}}$-valued Hida families in a similar manner to Definition 5.1.

5B2. Assumption TEMPERED. Let $\boldsymbol{f}$ be a Hida family of eigenforms as defined in Definition 5.1. We say it satisfies the assumption "TEMPERED" if the specializations $f_{\phi}$ in the definition are tempered eigenforms.

5B3. Assumption DUAL. We first define an $\widehat{O}_{L}$-involution $\circ: \Lambda \rightarrow \Lambda$ sending any $\operatorname{diag}\left(t_{1}, \ldots, t_{n}\right) \in T\left(1+\mathbb{Z}_{p}\right)$ to $\operatorname{diag}\left(t_{n}^{-1}, \ldots, t_{1}^{-1}\right)$. We define $\rrbracket^{\circ}$ to be the ring $\square$ but with the $\Lambda$-algebra structure given by composing the involution $\circ$ with the original $\Lambda$ structure map of $\square$.

Let $\boldsymbol{f}$ be an $\llbracket$-adic cuspidal eigenform on $\mathrm{U}(r, s)$ such that, for a Zariski-dense set of generic arithmetic points $\phi$, the specialization $\boldsymbol{f}_{\phi}$ is classical and generates an irreducible automorphic representation $\pi_{f_{\phi}}$ of $\mathrm{U}(r, s)$; we say it satisfies assumption DUAL if there is an $\square^{\circ}$-adic nearly ordinary cusp form $f^{\vee}$ on $\mathrm{U}(s, r)$ such that $f_{\phi}^{\vee} \in \pi_{f_{\phi}}^{\vee}$ for all the arithmetic points $\phi \in$ Spec $\llbracket$ that are in the image of some point in $\mathscr{Q}^{\text {gen }}$. (Here we identified $\mathrm{U}(r, s)$ and $\mathrm{U}(s, r)$ in the obvious way. At an arithmetic point both $f_{\phi}$ and $f_{\phi}^{\vee}$ have scalar weight $\kappa$. Note also that we only require the specialization $f_{\phi}$ to be "generic" (not required for $f_{\phi}^{\vee}$ ).)

5B4. Assumptions $\operatorname{Proj}_{f^{\vee}}$ and $\operatorname{Proj}_{f^{\vee}}$. We say a nearly ordinary cuspidal eigenform $f^{\vee}$ on $\mathrm{U}(s, r)$ satisfies assumption $\operatorname{Proj}_{f^{\vee}}$ if $\left(\pi_{f^{\vee}} \otimes W_{\Sigma \backslash\{p\}}\right)^{K}$ is 1-dimensional and there is a Hecke operator $1_{f^{\vee}}$ on $\mathrm{U}(s, r)$ that is an $L$-coefficient polynomial of Hecke operators outside $\Sigma$ such that, for any $g \in M_{\kappa}\left(K, W_{\Sigma \backslash\{p\}}\right)$, we have that $e^{\text {ord }} \cdot g-1_{f^{\vee}} e^{\text {ord }} \cdot g$ is a sum of forms in irreducible automorphic representations which are orthogonal to $\pi_{f}$. 
We say a nonzero nearly ordinary cuspidal $\mathbb{1}^{\circ}$-adic family of eigenforms $\boldsymbol{f}^{\vee}$ in $\left(V_{\infty, \infty}^{N}\left(K, \square^{\circ}, \chi_{0}^{-1}\right) \otimes W_{\Sigma \backslash\{p\}}\right)^{K_{\Sigma}}$ satisfies assumption $\operatorname{Proj}_{f^{\vee}}$ if there is an action $e^{\text {ord }}$ acting on $\left(V_{\infty, \infty}^{N}\left(K, \square^{\circ}, \chi_{0}^{-1}\right) \otimes W_{\Sigma \backslash\{p\}}\right)^{K_{\Sigma}}$ interpolating the $e^{\text {ord }}$ of specializations and there is a Hecke operator $1_{f} \vee$ which is an $F_{\square}$ polynomial of Hecke operators outside $\Sigma$ such that, for Zariski-dense set of arithmetic points $\phi \in$ Spec $\square^{\circ}$ in the image of $\mathscr{Q}^{\text {gen }},\left(\pi_{f_{\phi}^{\vee}} \otimes W_{\Sigma \backslash\{p\}}\right)^{K}$ is 1-dimensional and, for any $\boldsymbol{g} \in\left(V_{\infty, \infty}^{N}\left(K, \square^{\circ}, \chi_{0}^{-1}\right) \otimes W_{\Sigma}\right)^{K_{\Sigma}},\left(e^{\text {ord }} \cdot \boldsymbol{g}-1_{f^{\vee}} e^{\text {ord }} \boldsymbol{g}\right)_{\phi}$ is a sum of forms in irreducible automorphic representations which are orthogonal to $\pi_{f_{\phi}^{\vee}}$.

Remark 5.6. If $r+s=2$ then these assumptions often hold, since the unitary group is closely related to $\mathrm{GL}_{2}$ or quaternion algebras. It is easy to see DUAL by simply taking $f^{\vee}=f \otimes(\chi)^{-1}$ for $\chi$ the central character of $f$. To see $\operatorname{Proj}_{f}$ and $\operatorname{Proj}_{f^{\vee}}$, we first suppose $r=s=1$ and $f$ is a Hida family of $\mathrm{GL}_{2}$ newforms with tame level $M$ such that $\left(M, p \delta_{\mathscr{K}}\right)=1$ and trivial character. The existence of $e^{\text {ord }}$ is as in [Skinner and Urban 2014, Lemma 12.2] Since we have an isomorphism of algebraic groups over $F$,

$$
\mathrm{GU}(1,1) \sim \mathrm{GL}_{2} \times_{\mathbb{G}_{m}} \operatorname{Res} \mathscr{T}_{\digamma} / F \mathbb{G}_{m},
$$

we can obtain a family on $\mathrm{U}(1,1)$ from $f$ and the trivial character of $\mathbb{A}_{\mathscr{K}}^{\times} / \mathscr{K}^{\times}$, which we still denote by $\boldsymbol{f}$. Take an arithmetic point $\phi$ and a $\mathrm{GL}_{2}$ Hecke operator $t$ involving only Hecke operators $T_{v}$ at primes $v$ outside $\Sigma$ which are split in $\mathscr{K} / F$ such that the $t$-eigenvalue $t\left(f_{\phi}\right)$ is different from its eigenvalues on other forms on $S_{\kappa_{\phi}}^{\text {ord }}\left(\Gamma_{0}(M) \cap \Gamma_{1}\left(p^{t_{\phi}}\right), \mathbb{C}\right)$ (the space of ordinary cusp forms on $\mathrm{U}(1,1)$ of weight $\left(0, \kappa_{\phi}\right)$ and level $\Gamma_{0}(M) \cap \Gamma_{1}\left(p^{t_{\phi}}\right)$, with $p^{t_{\phi}}$ being the $p$-part level at $\phi$. Also here we use the $\mathrm{U}(1,1)$ Hecke operators at split primes $v=w \bar{w}$ which are associated to the elements $\left.\left(\operatorname{diag}\left(\varpi_{w}, 1\right), \operatorname{diag}\left(1, \varpi_{\bar{w}}^{-1}\right)\right)\right)$. This is possible since any form in $S_{\kappa_{\phi}}^{\text {ord }}\left(\Gamma_{0}(M) \cap \Gamma_{1}\left(p^{t_{\phi}}\right), \mathbb{C}\right)$ is the restriction of a form on $\operatorname{GU}(1,1)$ obtained from a $\mathrm{GL}_{2}$ form of conductor dividing $\mathrm{Nm}_{\mathscr{K} / F} \delta_{\mathscr{K} / F} M p^{t_{\phi}}$ and a character of $\mathbb{A}_{\mathscr{K}}^{\times} / \mathscr{K}^{\times}$ unramified outside $p$. Note that any cuspidal automorphic representation on $\mathrm{GL}_{2} / F$ with the same Hecke eigenvalue with $\boldsymbol{f}_{\phi}$ on split primes is $\pi_{f_{\phi}}$ or $\pi_{f_{\phi}} \otimes \chi_{\mathscr{K} / F}$, and that any element $g \in \mathrm{GL}_{2}\left(F_{v}\right)$ such that $\operatorname{det}(g) \in \mathrm{Nm}_{\mathscr{K} / F}\left(\mathscr{K}_{v}^{\times}\right)$can be written as $a g^{\prime}$ with $a \in \mathscr{K}_{v}^{\times}$and $g^{\prime} \in \mathrm{U}(1,1)\left(F_{v}\right)$. A simple representation-theoretic argument shows that the only forms in $S_{\kappa_{\phi}}^{\text {ord }}\left(\Gamma_{0}(M) \cap \Gamma_{1}\left(p^{t_{\phi}}\right), \mathbb{C}\right)$ with the same Hecke eigenvalues with $\boldsymbol{f}_{\phi}$ at split primes are in the 1-dimensional space spanned by $\boldsymbol{f}_{\phi}$. Let $\Lambda$ be the weight space for $\mathrm{U}(1,1)$ and define

$$
S^{\text {ord }}\left(\Gamma_{0}(M), \rrbracket\right):=S^{\text {ord }}\left(\Gamma_{0}(M), \Lambda\right) \otimes_{\Lambda} \rrbracket .
$$

It follows from Hida's control theorem for unitary groups (see [Hsieh 2014, The-

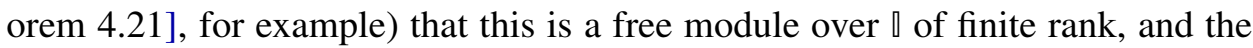
specialization of this free module to $\phi$ gives the space $S_{\kappa_{\phi}}^{\text {ord }}\left(\Gamma_{0}(M) \cap \Gamma_{1}\left(p^{t_{\phi}}\right), O_{L}\right)$ for some $L$ finite over $\mathbb{Q}_{p}$ provided $\kappa_{\phi} \gg 0$ with respect to the $p$-part of the conductor 
of $\phi$. We consider $\operatorname{det}(T-t)$, where $T$ is a variable and we regard $t$ as an operator on this free $\llbracket$-module. We thus obtain an $\square$-coefficient polynomial of $T$. Moreover, we can write $\operatorname{det}(T-t)=(T-t(f)) \cdot g(T)$ for some polynomial $g(T)$. Then we define

$$
1_{f}=\frac{g(t)}{g(f)}
$$

(note that $g(t(f))$ is not identically zero.) This proves $\operatorname{Proj}_{f}$, and $\operatorname{Proj}_{f^{\vee}}$ is seen in a similar way. If $(r, s)=(2,0)$ we observe that if we set

$$
D=\left\{g \in M_{2}(\mathscr{K}) \mid g^{t} \zeta \bar{g}=\operatorname{det}(g) \zeta\right\}
$$

then $D$ is a definite quaternion algebra over $\mathbb{Q}$ with local invariants $\operatorname{inv}_{v}(D)=$ $\left(-\mathfrak{s},-D_{\mathscr{K} / \mathbb{Q}}\right)_{v}$ (the Hilbert symbol). The relation between $\mathrm{GU}(2)$ and $D$ is explained by

$$
\mathrm{GU}(2)=D^{\times} \times_{\mathbb{G}_{m}} \operatorname{Res} \mathscr{K} / \mathbb{Q} \mathbb{G}_{m} .
$$

We can similarly show that, if $f$ is a Hida family of newforms on $D^{\times}$with trivial character, tame level prime to $p$ and all primes of $\delta_{\mathscr{H}}$ such that $D$ is unramified, and is the trivial representation at primes where $D$ is ramified, then we can produce a family $\boldsymbol{f}$ on $\mathrm{U}(2,0)$ from $\boldsymbol{f}$ and the trivial character of $\mathbb{A}_{\mathscr{K}}^{\times} / \mathscr{K}^{\times}$. A similar argument proves that $\operatorname{Proj}_{f}$ and $\operatorname{Proj}_{f} \vee$ is true.

\section{C. Klingen Eisenstein series and p-adic L-functions.}

5C1. Construction. Now we are going to construct the nearly ordinary Klingen Eisenstein series (and will $p$-adically interpolate them in families). First of all, let $\tau$ be a Hecke character which is of infinite type $\left(-\frac{1}{2} \kappa, \frac{1}{2} \kappa\right)$ at all infinite places (here the convention is that the first infinite place of $\mathscr{K}$ is inside our CM type). Recall that we write $\mathscr{D}:=\{\pi, \tau, \Sigma\}$ for the Eisenstein data (see Definition 3.2). We define the normalization factor

$$
\begin{aligned}
B_{\mathscr{D}}:= & \frac{\Omega_{p}^{r \kappa \Sigma_{\infty}}}{\Omega_{\infty}^{r \kappa \Sigma_{\infty}}}\left(\frac{(-2)^{-d(a+2 b+1)}(2 \pi i)^{d(a+2 b+1) \kappa}(2 / \pi)^{d(a+2 b+1)(a+2 b) / 2}}{\prod_{j=0}^{a+2 b}(\kappa-j-1)^{d}}\right)^{-1} \\
& \times \prod_{i=0}^{a+2 b} L^{\Sigma}\left(2 z_{\kappa}+a+2 b+1-i, \bar{\tau}^{\prime} \chi_{\mathscr{K}}^{i}\right) \prod_{v \mid p}\left(\mathfrak{g}\left(\bar{\tau}_{v}^{\prime}\right)^{a+2 b+1} c_{a+2 b+1}\left(\tau_{v}^{\prime},-z_{\kappa}\right)\right)^{-1}, \\
B_{\mathscr{D}}^{\prime}:= & \frac{\Omega_{p}^{r \kappa \Sigma_{\infty}}}{\Omega_{\infty}^{r \kappa \Sigma_{\infty}}}\left(\frac{(-2)^{-d(a+2 b)}(2 \pi i)^{d(a+2 b) \kappa}(2 / \pi)^{d(a+2 b)(a+2 b-1) / 2}}{\prod_{j=0}^{a+2 b-1}(\kappa-j-1)^{d}}\right)^{-1} \\
& \times \prod_{i=0}^{a+2 b-1} L^{\Sigma}\left(2 z_{\kappa}+a+2 b-i, \bar{\tau}^{\prime} \chi_{\mathscr{K}}^{i}\right) \prod_{v \mid p}\left(\mathfrak{g}\left(\bar{\tau}_{v}^{\prime}\right)^{a+2 b} c_{a+2 b}\left(\tau_{v}^{\prime},-z_{\kappa}^{\prime}\right)\right)^{-1} .
\end{aligned}
$$

Here, $z_{\kappa}=\frac{1}{2}(\kappa-a-2 b-1)$ and $z_{\kappa}^{\prime}=\frac{1}{2}(\kappa-a-2 b), c_{m}$ is defined in (13), and $\Omega_{\infty}$ is the CM period in Section 2A. 
We construct a Siegel Eisenstein series $E_{\text {sieg }}$ associated to the Siegel section

$$
f_{\mathscr{D}, \text { sieg }}=B_{\mathscr{D}} \prod_{v \mid \infty} f_{\kappa} \prod_{v \mid p} \rho\left(\Upsilon_{v}\right) f_{v}^{0} \prod_{v \in \Sigma, v \nmid p} \tilde{f}_{v, \text { sieg }} \prod_{v} f_{v}^{\mathrm{sph}} \in I_{a+2 b+1}(\tau, z)
$$

and $E_{\text {sieg }}^{\prime}$ associated to the section

$$
f_{\mathscr{D}, \text { sieg }}^{\prime}=B_{\mathscr{D}}^{\prime} \prod_{v \mid \infty} f_{\kappa}^{\prime} \prod_{v \mid p} \rho\left(\Upsilon_{v}^{\prime}\right) f_{v}^{0 \prime} \prod_{v \in \Sigma, v \nmid p} \tilde{f}_{v, \text { sieg }}^{\prime} \prod_{v} f_{v}^{\mathrm{sph}, \prime} \in I_{a+2 b}(\tau, z) .
$$

Here $\Upsilon_{v}$ and $\Upsilon_{v}^{\prime}$ are as defined in Definition 4.32. First note that, since $\pi$ is nearly ordinary with respect to the scalar weight $\kappa$, its contragradient is also nearly ordinary on $\mathrm{U}(s, r)$ with respect to the scalar weight $\kappa$. We denote this representation by $\tilde{\pi}$. We consider $E(\gamma(g,-))$ as an automorphic form on $\mathrm{U}(s, r)$. For each $v \nmid p$ we choose an open compact group $\tilde{K}_{v, s} \subset \mathrm{U}(s, r)_{v}$ such that

$$
\prod_{v \in \Sigma, v \nmid p} \rho\left(\gamma\left(1, \eta \operatorname{diag}\left(\bar{x}_{v}^{-1}, 1, x_{v}\right) . \tilde{S}_{v}^{-1}\right)\right)(E(\gamma(g,-)) \otimes \bar{\tau}(\operatorname{det}-))
$$

is invariant under its action. We have the following lemma:

Lemma 5.7. There is a bounded measure $\mathscr{E}_{\mathscr{D}, \text { sieg }}$ on $\Gamma_{\mathscr{C}} \times T\left(1+\mathbb{Z}_{p}\right)$ with values in the space of $p$-adic automorphic forms on $\mathrm{U}(r+s+1, r+s+1)$ such that, for all arithmetic points $\phi \in \mathscr{X}^{\text {gen }}$ with the associated character $\hat{\phi}$ on $\Gamma_{\mathscr{K}} \times T\left(1+\mathbb{Z}_{p}\right)$, we have

$$
\int_{\Gamma_{\mathscr{K}} \times T\left(1+\mathbb{Z}_{p}\right)} \hat{\phi} d \mathscr{E} \mathscr{D}, \text { sieg }
$$

is the Siegel Eisenstein series $\rho\left(\prod_{v \in \Sigma, v \nmid p} \gamma\left(1, \eta \operatorname{diag}\left(\bar{x}_{v}^{-1}, 1, x_{v}\right) \tilde{S}_{v}^{-1}\right)\right) E_{\text {sieg, } \mathscr{D}_{\phi}}$, where $E_{\mathrm{sieg}, \mathscr{D}_{\phi}}$ is the Siegel Eisenstein series we construct using the characters $\left(\chi_{1, \phi}, \ldots, \chi_{n, \phi}, \tau_{\phi}\right)$. Similarly, we can define a measure $\mathscr{E}^{\prime}{ }_{\text {, sieg }}$ interpolating the $E_{\text {sieg, } \mathscr{D}_{\phi}}^{\prime}$.

Proof. It follows from our computations for Fourier coefficients, Lemmas 4.2, 4.6, 4.12 and 4.46, and [Skinner and Urban 2014, Lemma 11.2], that all the Fourier coefficients of $E_{\text {sieg }}$ and $E_{\text {sieg }}^{\prime}$ are interpolated by elements in $\Lambda_{r, s} \llbracket \Gamma_{\mathscr{K}} \rrbracket$. Then the lemma follows from the abstract Kummer congruence. We refer to [Hsieh 2011, Lemma 3.15, Theorem 3.16] for a detailed proof.

Now we define our Klingen Eisenstein series using the pullback formula. Note that by (3) the pullback of the Siegel Eisenstein series are still holomorphic automorphic forms. Let $\beta$ be the embedding given in Section 2B. Let $\tilde{K}_{s}$ be the open compact subgroup of $G\left(\mathscr{O}_{F, \Sigma}\right)$, which is $\tilde{K}_{v, s}$ as above for $v \in \Sigma \backslash\{v \mid p\}, \tilde{K}_{v}$ for $v \mid p$ and spherical otherwise. We define $\boldsymbol{E}_{\boldsymbol{D} \text {, Kling }}$ by, for any points $x$ and $x_{1}$ on the 
Igusa schemes of $\mathrm{U}(r+1, s+1)$ and $\mathrm{U}(s, r)$, $e^{\text {ord, low }} \cdot 1_{\boldsymbol{f}^{\vee}}^{\text {low }} \operatorname{Tr}_{\tilde{K} / \tilde{K}_{s}}\left(e^{\text {low }}\left(\beta^{-1}\left(\mathscr{E}_{\boldsymbol{D}, \text { sieg }}\right) \cdot \bar{\tau}\left(\operatorname{det}\left(g_{1}\right)\right)\right) \otimes v_{1}\right)\left(x, x_{1}\right)$

$$
=\boldsymbol{E}_{\boldsymbol{D}, \mathrm{Kling}}(x) \otimes \boldsymbol{f}^{\vee}\left(x_{1}\right)
$$

(as a $W_{\Sigma \backslash\{p\}}$-valued form - recall $v_{1} \in W_{\Sigma \backslash\{p\}}$; see Section 5B1). Here we let $\tilde{K}_{\Sigma \backslash\{p\}}$ act on both $\mathscr{E}_{D}$, sieg and $W_{\Sigma \backslash\{p\}}$. We get a $\Lambda_{\mathscr{D}}$-adic formal Fourier-Jacobi expansion from the measure $e^{\text {low }} \beta^{-1}\left(\mathscr{E}_{D}\right.$, sieg $)$ and then apply the Hecke operators to the expansion. We also define the $\Sigma$-primitive $p$-adic $L$-function $\mathscr{L}_{\boldsymbol{f}, \mathscr{K}, \tau_{0}}^{\Sigma} \in \mathbb{\natural}^{\mathrm{ur}} \llbracket \Gamma_{\mathscr{K}} \rrbracket$ by, for elements $x$ and $x_{1}$ in the Igusa schemes of $\mathrm{U}(r, s)$ and $\mathrm{U}(s, r)$,

$$
\begin{aligned}
e^{\text {ord,low }} \cdot 1_{\boldsymbol{f}^{\vee}}^{\text {low }} \operatorname{Tr}_{\tilde{K} / \tilde{K}_{s}}\left(e^{\text {low }} \beta^{-1}\left(\mathscr{E}_{D}^{\prime} \text {, sieg }\right) \cdot \bar{\tau}\left(\operatorname{det} g_{1}\right) \otimes v_{1}\right)\left(x, x_{1}\right) & \\
& =\mathscr{L}_{\boldsymbol{f}, \mathscr{K}, \tau_{0}}^{\Sigma} \boldsymbol{f}_{1}(x) \otimes \boldsymbol{f}^{\vee}\left(x_{1}\right) .
\end{aligned}
$$

The $\boldsymbol{f}_{1}$ is the $v_{1}^{\vee}$-component of $\boldsymbol{f}$ (see Section 5B1). This is possible by Lemma 5.4. Here note that the necessity of enlarging the coefficient ring to include $O_{L}^{\mathrm{ur}}$ is caused when specifying points on Igusa schemes (recall Section $2 \mathrm{~F}$ ).

Here we used the superscript "low" to mean that, under

$$
\mathrm{U}(a+b+1, b+1) \times \mathrm{U}(b, a+b) \hookrightarrow \mathrm{U}(a+2 b+1, a+2 b+1),
$$

the action is for the group $\mathrm{U}(b, a+b)$.

5C2. Identify with Klingen Eisenstein series constructed before. We define a Klingen Eisenstein section by

$$
f_{\mathscr{D}_{\phi}, \text { Kling }}^{\square}(z, g)=B_{\mathscr{D}} \prod_{v} F_{\varphi_{v}}\left(z ; f_{v, \text { sieg }}, g\right),
$$

where the $F_{\varphi_{v}}\left(z ; f_{v \text {,sieg }}, g\right)$ are the pullback sections we computed in Section 5 and $\varphi_{v}$ for $v \in \Sigma \backslash\{v \mid p\}$ is the $v_{1}^{\vee}$-component, as in Sections 5B1 and 5B4. We first look at places dividing $p$. The pairing $\langle$,$\rangle induces a natural pairing between \pi$ and $\tilde{\pi}$. Write

$$
\varphi_{w}=\prod_{v \mid \infty} \varphi_{v} \prod_{v \notin \Sigma} \varphi^{\mathrm{sph}} \prod_{v \in \Sigma, v \nmid p} \varphi_{v} \prod_{v \mid p} \varphi_{w, v} .
$$

Then

$$
\begin{aligned}
& \left\langle\prod_{v \nmid p} \operatorname{Tr}_{\tilde{K}_{v} / \tilde{K}_{v}, s} \rho\left(\gamma\left(1, \eta \operatorname{diag}\left(\bar{x}_{v}^{-1}, 1, x_{v}\right) . \tilde{S}_{v}^{-1}\right)\right)\left(E_{\text {sieg }}(\gamma(g,-)) \bar{\tau}(\operatorname{det}-)\right),\right. \\
& \left.\quad \rho\left(\prod_{v \mid p}\left(\operatorname{diag}\left(p^{-t_{a+b+1}}, \ldots, p^{t_{1}}, \ldots, p^{t_{a+1}}, \ldots\right)^{\iota}\left(1_{1_{b}}^{-1_{b}}\right)^{\iota}\right)\right) \varphi_{w}\right\rangle
\end{aligned}
$$


equals

$$
\begin{aligned}
\left\langle\rho^{\operatorname{low}}\left(\prod_{v \mid p} \operatorname{diag}\left(p^{t_{a+b+1}}, \ldots, p^{t_{a+2 b}}, 1_{a}, 1_{b}\right)^{\iota}\right)\right. \\
\times \prod_{v \nmid p} \operatorname{Tr}_{\tilde{K}_{v} / \tilde{K}_{v}, s} \rho\left(\gamma\left(1, \eta \operatorname{diag}\left(\bar{x}_{v}^{-1}, 1, x_{v}\right) \tilde{S}_{v}^{-1}\right)\right)(E(\gamma(g,-)) \bar{\tau}(\operatorname{det}-)), \\
\left.\quad \rho\left(\prod_{v \mid p}\left(\operatorname{diag}\left(1_{b}, p^{t_{1}}, \ldots, p^{t_{a+1}}, \ldots\right)^{\iota}\left(v_{1^{\prime}} 1^{-1_{b}}\right)\right)\right) \varphi_{w}\right\rangle .
\end{aligned}
$$

Since $E_{\text {sieg }}(\gamma(g,-)) \bar{\tau}($ det -$)$ satisfies the property that, if $\tilde{K}^{\prime \prime \prime}$ is the subgroup of $\mathrm{GL}_{a+2 b}\left(\mathbb{Z}_{p}\right)$ (defined in the last section) consisting of matrices

$$
\left(\begin{array}{lll}
a_{1} & a_{3} & a_{2} \\
a_{7} & a_{9} & a_{8} \\
a_{4} & a_{6} & a_{5}
\end{array}\right)
$$

such that the $(i, j)$-th entries of $a_{7}$ and $a_{4}$ are divisible by $p^{t_{i}+t_{a+b+j}}$ and $p^{t_{a+i}+t_{a+b+j}}$, respectively, the $i$-th row of $a_{8}$ and the right-to-diagonal entries of $a_{9}$ are divisible by $p^{t_{i}}$ for $i=1, \ldots, a$, the below-diagonal entries of the $i$-th column of $a_{1}$ are divisible by $p^{t_{a+b+i}}$, the up-to-diagonal entries of the $i$-th row of $a_{5}$ are divisible by $p^{t_{a+i}}$, and $a_{2}, a_{3}, a_{6} \in M\left(\mathbb{Z}_{p}\right)$, then the right action of $h^{\iota}$ for $h \in \tilde{K}^{\prime \prime \prime}$ on $E(\gamma(g,-)) \bar{\tau}(\operatorname{det}-)$ is given by the character

$$
\begin{aligned}
\lambda\left(h^{\iota}\right)=\bar{\chi}_{a+b+1}\left(h_{11}\right) \cdots \bar{\chi}_{a+2 b}\left(h_{b b}\right) \bar{\chi}_{1}\left(h_{b+1, b+1}\right) \cdots \bar{\chi}\left(h_{a+b, a+b}\right) \\
\times \bar{\chi}_{a+1}\left(h_{a+b+1, a+b+1}\right) \cdots \bar{\chi}_{a+b}\left(h_{a+2 b, a+2 b}\right) .
\end{aligned}
$$

(This is easily checked from the definition of the Godement section.) It is elementary to check that the above expression equals

$\prod_{v \mid p} \frac{1}{\prod_{i=1}^{b} p^{t_{a+b+i}(a+b)}}$

$$
\begin{aligned}
& \times\left\langle\prod_{v \mid p} \sum_{y} \rho^{\text {low }}(y) \rho^{\text {low }}\left(\operatorname{diag}\left(p^{t_{a+b+1}}, \ldots, 1_{a}, 1_{b}\right)^{\iota}\right)\right. \\
& \times \prod_{v \nmid p} \operatorname{Tr}_{\tilde{K}_{v} / \tilde{K}_{v}, s} \rho\left(\gamma\left(1, \eta \operatorname{diag}\left(\bar{x}_{v}^{-1}, 1, x_{v}\right) \tilde{S}_{v}^{-1}\right)\right)\left(E_{\text {sieg }}(\gamma(g,-)) \bar{\tau}(\operatorname{det}-)\right), \\
& \left.\rho\left(\prod_{v \mid p} \operatorname{diag}\left(1_{b}, p^{t_{1}} \ldots, p^{t_{a+1}}, \ldots\right)^{\iota}\left(1_{1_{b}}{ }^{-1_{b}}\right)^{\iota}\right) \varphi_{w}\right\rangle
\end{aligned}
$$


where $y$ runs over $N\left(\mathbb{Z}_{p}\right) / \beta N\left(\mathbb{Z}_{p}\right) \beta^{-1}$ for $N$ consisting of matrices of the form $\left(\begin{array}{cc}1_{s} & 0 \\ * & 1_{r}\end{array}\right)$ with $*$ having $\mathbb{Z}_{p}$-entries and $\beta=\operatorname{diag}\left(p^{t_{a+b+1}}, \ldots, 1_{a}, 1_{b}\right)$. Write the expression

$$
\begin{aligned}
& e^{\text {ord,low }} \prod_{v \mid p} \sum_{y} \rho^{\text {low }}(y) \rho^{\text {low }}\left(\beta^{\iota}\right) \\
& \quad \times \prod_{v \nmid p} \operatorname{Tr}_{\tilde{K}_{v} / \tilde{K}_{v}, s} \rho\left(\gamma\left(1, \eta \operatorname{diag}\left(\bar{x}_{v}^{-1}, 1, x_{v}\right) \tilde{S}_{v}^{-1}\right)\right)\left(E_{\text {sieg }}(\gamma(g,-)) \bar{\tau}(\operatorname{det}-)\right) .
\end{aligned}
$$

Now let $\tilde{K}^{b}$ consists of matrices in $\mathrm{GL}_{b}\left(\mathbb{Z}_{p}\right)$ whose below-diagonal entries of the $i$-th row are divisible by $p_{a+b+i}^{t}$ for $1 \leq i \leq s$. Let $\tilde{K}^{\sharp}$ be the set of elements in $\mathrm{GL}_{a+2 b}\left(\mathbb{Z}_{p}\right)$ whose right-to-diagonal entries of the $i$-th row are divisible by $p^{t_{i}}$ for $1 \leq i \leq a+b$ and whose lower-right $b \times b$ block is in

$$
\operatorname{diag}\left(p^{t_{a+b+1}}, \ldots, p^{t_{a+2 b}}\right) \tilde{K}^{b} \operatorname{diag}\left(p^{t_{a+b+1}}, \ldots, p^{t_{a+2 b}}\right)^{-1} .
$$

Then a similar argument as in Section 4D1 shows that there is a unique (up to scalar) vector $\tilde{\varphi}_{v}^{\sharp} \in \pi\left(\chi_{1}^{-1}, \ldots, \chi_{a+2 b}^{-1}\right)$ such that the action of $\left(k_{i j}\right) \in K^{\sharp}$ is given by the character $\operatorname{diag}\left(\chi_{1}^{-1}\left(k_{11}\right), \ldots, \chi_{a+2 b}^{-1}\left(k_{a+2 b a+2 b}\right)\right)$. We use the model of the induced representation from $\chi_{1}^{-1} \otimes \cdots \otimes \chi_{a+2 b}^{-1}$ on the space of smooth functions on $\mathrm{GL}_{a+2 b}\left(\mathbb{Z}_{p}\right)$. We take $\tilde{\varphi}_{v}^{\sharp}$ such that, if $\tilde{\varphi}_{v}^{\text {ord }}$ takes value 1 on identity in this model, then $\tilde{\varphi}_{v}^{\sharp}$ also takes value 1 on identity (and has support $K^{\sharp} \subset \mathrm{GL}_{a+2 b}\left(\mathbb{Z}_{p}\right)$ ). From the action of the level group we know that the action of $\rho^{\text {low }}\left(K^{\sharp}\right)$ on the left part of the inner product in (15) is given by the character $\operatorname{diag}\left(\chi_{1}^{-1}\left(k_{11}\right), \ldots, \chi_{a+2 b}^{-1}\left(k_{a+2 b a+2 b}\right)\right)$. For $v \mid p$ define $T_{\beta, v}^{\text {low }}$ to be the Hecke operator corresponding to $\beta$ just in terms of double cosets acting on $\pi_{\varphi}^{\vee}$ (with no normalization factors involved). By checking the actions of the level groups at primes dividing $p$ (certain open compact subgroups of $G\left(\mathcal{O}_{F, p}\right)$ ) we can see that the $\tilde{\pi}$ component of the left part, when viewed as an automorphic form on $\mathrm{U}(a+b, b)$, is a multiple of $\tilde{\varphi}^{\text {ord }}$. Suppose the eigenvalue for the Hecke operator $T_{\beta, v}^{\text {low }}$ on $\tilde{\varphi}^{\text {ord }}$ is $\tilde{\lambda}_{\beta, v}$. It is easy to compute that

$$
\tilde{\lambda}_{\beta, v}=p^{\sum_{i=1}^{b} t_{a+b+i}((a+2 b+1) / 2-i)} \cdot \prod_{j=1}^{b} \chi_{a+2 b+1-j}^{-1}\left(p^{t_{a+b+j}}\right)
$$

with the convention on the $\chi_{i}$ after Remark 4.41.

Let

$$
\begin{aligned}
\varphi^{\prime}=\prod_{v \mid \infty} \varphi_{v} & \prod_{v \notin \Sigma} \varphi^{\mathrm{sph}} \prod_{v \in \Sigma, v \nmid p} \varphi_{v} \\
& \times \prod_{v \mid p} \rho\left(\operatorname{diag}\left(p^{-t_{a+b+1}}, \ldots, p^{t_{1}}, \ldots, p^{t_{a+1}}, \ldots\right)^{\iota}\left(\begin{array}{c}
1_{a}-1_{b} \\
1^{b}
\end{array}\right)^{\iota}\right) \varphi_{w, v}
\end{aligned}
$$


and

$$
\begin{aligned}
\varphi^{\prime \prime}=\prod_{v \mid \infty} \varphi_{v} \prod_{v \notin \Sigma} \varphi^{\mathrm{sph}} \prod_{v \in \Sigma, v \nmid p} \varphi_{v} \\
\quad \times \prod_{v \mid p} \rho\left(\operatorname{diag}\left(1, \ldots, p^{t_{1}}, \ldots, p^{t_{a+1}}, \ldots\right)^{\iota}\left(1_{a}^{-1_{b}}\right)^{\iota}\right) \varphi_{w, v} .
\end{aligned}
$$

Here, for $v \mid \infty$, the $\varphi_{v}$ is the unique vector mentioned before Definition 3.1. Define the Klingen Eisenstein section promised in the introduction as

$$
f_{\mathscr{D}_{\phi}, \mathrm{Kling}}=B_{\mathscr{D}_{\phi}}\left(\prod_{v \in \Sigma, v \nmid p}\left|\tilde{K}_{v} / \tilde{K}_{v, s}\right|\right) f_{\mathscr{D}_{\phi}, \mathrm{Kling}}^{\square} \cdot
$$

Then we have:

Proposition 5.8. For a classical generic arithmetic point $\phi$, we have $\phi\left(\boldsymbol{E}_{\boldsymbol{D}, \mathrm{Kling}}\right)$

$$
\begin{aligned}
= & \prod_{v \in \Sigma, v \nmid p}\left|\tilde{K}_{v} / \tilde{K}_{v, s}\right| \frac{E_{\mathrm{Kling}}\left(f_{\mathscr{D}_{\phi}, \mathrm{Kling}}, z_{\kappa_{\phi}}, g\right)}{\left\langle\tilde{\varphi}_{\phi}^{\text {ord }}, \varphi_{\phi}\right\rangle} \\
& \times \prod_{v \mid p}\left(\prod_{j=1}^{s} \chi_{r+j}\left(p^{t_{r+j}}\right) \prod_{j=1}^{r} \chi_{j}^{-1}\left(p^{t_{j}}\right) p^{\sum_{i=1}^{s} t_{a+b+i}((a-1) / 2+i)} \cdot p^{-\sum_{j=1}^{r} t_{j}((a+1) / 2-j)}\right) .
\end{aligned}
$$

Proof. Here, let $\Theta$ be the expression (15) and $\Xi$ the expression (16). We have

$$
\frac{\Theta}{\left\langle\tilde{\varphi}^{\sharp}, \varphi^{\prime \prime}\right\rangle}=\frac{\left\langle\Xi, \varphi^{\prime \prime}\right) \tilde{\lambda}_{\beta, v}}{\prod_{v \mid p}\left(\prod_{1 \leq i \leq j \leq s} p^{t_{a+b+i}-t_{a+b+j}}\right)\left\langle\tilde{\varphi}^{\text {ord }}, \varphi^{\prime \prime}\right\rangle}
$$

and

$$
\left\langle\tilde{\varphi}^{\sharp}, \varphi^{\prime \prime}\right\rangle=\left\langle\tilde{\varphi}^{\text {ord }}, \varphi^{\prime \prime}\right\rangle \cdot \prod_{v \mid p}\left(\prod_{1 \leq i \leq j \leq s} p^{t_{a+b+i}-t_{a+b+j}}\right)
$$

(e.g., using the model of the induced representation). So

$$
\begin{aligned}
\frac{\left\langle\Xi, \varphi^{\prime \prime}\right\rangle}{\left\langle\tilde{\varphi}^{\text {ord }}, \varphi^{\prime \prime}\right\rangle} & =\frac{\Theta \prod_{v \mid p}\left(\prod_{1 \leq i \leq j \leq s} p^{t_{a+b+i}-t_{a+b+j}}\right)}{\tilde{\lambda}_{\beta, v}\left\langle\tilde{\varphi}^{\sharp}, \varphi^{\prime \prime}\right\rangle} \\
& =\frac{\Theta \prod_{v \mid p}\left(\prod_{1 \leq i \leq j \leq s} p^{t_{a+b+i}-t_{a+b+j}}\right)}{\prod_{v \mid p}\left(\prod_{1 \leq i \leq j \leq s} p^{t_{a+b+i}-t_{a+b+j}}\right) \tilde{\lambda}_{\beta, v}\left\langle\tilde{\varphi}^{\text {ord }}, \varphi^{\prime \prime}\right\rangle} .
\end{aligned}
$$

We also have

$$
\left\langle\tilde{\varphi}^{\text {ord }}, \varphi^{\prime \prime}\right\rangle=\prod_{j=1}^{r} \chi_{j}\left(p^{t_{j}}\right) \cdot p^{\sum_{j=1}^{r} t_{j}((a+1) / 2-j)} .
$$

The proposition follows. 
Then parts (i) and (ii) of Theorem 1.1 are just a corollary of the above proposition (except the statement in the $s=0$ case, which we are going to consider next).

Similarly, we obtain an interpolation formula for the $p$-adic $L$-function as in Theorem 1.1, using also the formula (15).

5C3. Interpolating Petersson inner products for definite unitary groups. To simplify the exposition we only discuss the case when $F=\mathbb{Q}$ in this subsubsection. In the case when $s=0$, we hope that the periods showing up are CM periods. Thus, by our assumption, the Archimedean components of $\pi$ are trivial representations. For this purpose we prove that, under certain assumptions, the Petersson inner products of two families can be interpolated by elements in the Iwasawa algebra. Let $K=\prod_{v} K_{v}$ be an open compact subgroup of $\mathrm{U}(r, s)\left(\mathbb{A}_{f}\right)$ which is $G\left(\mathbb{Z}_{p}\right)$ at all primes dividing $p$ and $K_{0}(p)$, obtained from $K$ by replacing the $v$-component by $K_{0}^{1}$ at all primes $v$ dividing $p$. Now we take a set $\left\{g_{i}\right\}_{i}$ of representatives for $\mathrm{U}(r, s)(F) \backslash \mathrm{U}(r, s)\left(\mathbb{A}_{F}\right) / K_{0}(p)$. We take $K$ sufficiently small so that for all $i$ we have $\mathrm{U}(r, s)(F) \cap g_{i} K g_{i}^{-1}=1$. For the nearly ordinary Hida family $f^{\vee}$ of eigenforms (recall that this Hida family is nearly ordinary with respect to the lower-triangular Borel subgroup) we construct a bounded !-valued measure $\mu_{i}$ on $N^{-}\left(p \mathbb{Z}_{p}\right)$ as follows. Let $T^{-}$be the set of elements $\operatorname{diag}\left(p^{a_{1}}, \ldots, p^{a_{r}}\right)$ with $a_{1} \leq \cdots \leq a_{r}$. We only need to specify the measure for sets of the form $n t^{-} N^{-}\left(\mathbb{Z}_{p}\right)\left(t^{-}\right)^{-1}$, where $n \in N^{-}\left(\mathbb{Z}_{p}\right)$ and $t^{-} \in T^{-}$. We assign its measure $\mu_{i}\left(n t^{-} N^{-}\left(\mathbb{Z}_{p}\right)\left(t^{-}\right)^{-1}\right)$ by $f^{\vee}\left(g_{i} n \cdot t^{-}\right) \lambda\left(t^{-}\right)^{-1}$, where $\lambda\left(t^{-}\right)$is the Hecke eigenvalue of $f^{\vee}$ for $U_{t^{-}}$. This does define a measure. We briefly explain the point when $r=2$ (the general case is only notationally more complicated). Write $\pi_{f_{\phi}^{\vee}, p}=\pi\left(\chi_{1, p}, \chi_{2, p}\right)$ such that $v_{p}\left(\chi_{1, p}(p)\right)=\frac{1}{2}, v_{p}\left(\chi_{2, p}(p)\right)=-\frac{1}{2}$. Then $\lambda\left(\operatorname{diag}\left(1, p^{n}\right)\right)=\left(\chi_{2, p}(p) \cdot p^{1 / 2}\right)^{n}$. One checks that

$$
\begin{aligned}
\sum_{m \in p^{n-1} \mathbb{Z}_{p} / p^{n} \mathbb{Z}_{p}} \pi\left(\left(\begin{array}{cc}
1 & \\
m & 1
\end{array}\right)_{p}\right) \pi\left(\operatorname{diag}\left(1, p^{n}\right)_{p}\right) \boldsymbol{f}_{\phi, p}^{\vee} \\
=\left(\chi_{2, p}(p) \cdot p^{1 / 2}\right) \pi\left(\operatorname{diag}\left(1, p^{n-1}\right)_{p}\right) \boldsymbol{f}_{\phi, p}^{\vee} .
\end{aligned}
$$

This implies that, for any $m_{1} \in p \mathbb{Z}_{p} / p^{n-1} \mathbb{Z}_{p}$,

$$
\begin{aligned}
\sum_{m_{2} \in p^{n-1} \mathbb{Z}_{p} / p^{n} \mathbb{Z}_{p}} \mu_{i}\left(m_{1} m_{2} \operatorname{diag}\left(1, p^{n}\right) N^{-}\left(\mathbb{Z}_{p}\right) \operatorname{diag}\left(1, p^{-n}\right)\right) \\
=\mu_{i}\left(m_{1} \operatorname{diag}\left(1, p^{n-1}\right) N^{-}\left(\mathbb{Z}_{p}\right) \operatorname{diag}\left(1, p^{1-n}\right)\right),
\end{aligned}
$$

i.e., this $\mu_{i}$ does define a measure.

Proposition 5.9. If we define

$$
\left\langle\boldsymbol{f}, \boldsymbol{f}^{\vee}\right\rangle:=\sum_{i} \int_{n \in N^{-}\left(p \mathbb{Z}_{p}\right)} \boldsymbol{f}\left(g_{i} n\right) d \mu_{i} \in \mathbb{}
$$

then, for all $\phi \in \mathscr{X}^{\text {gen }}$, the specialization of $\left\langle\boldsymbol{f}, \boldsymbol{f}^{\vee}\right\rangle$ to $\phi$ is $\left\langle\boldsymbol{f}_{\phi}, \boldsymbol{f}_{\phi}^{\vee}\right\rangle \cdot \operatorname{Vol}\left(\tilde{K}_{\phi}\right)^{-1}$. 
Proof. For each $\phi \in \mathscr{Q}^{\text {gen }}$, we choose $t^{-}$such that $t^{-} N^{-}\left(p \mathbb{Z}_{p}\right)\left(t^{-}\right)^{-1} \subseteq \tilde{K}_{\phi}$. We consider

$$
\left\langle\boldsymbol{f}_{\phi}, \pi_{\boldsymbol{f}_{\phi}}^{\vee}\left(t^{-}\right) \boldsymbol{f}_{\phi}^{\vee}\right\rangle
$$

Unfolding the definitions, note $\chi_{\phi}^{-1}\left(t^{-}\right) \delta_{B}\left(t^{-}\right)$gives the Hecke eigenvalue $\lambda\left(t^{-}\right)$; this gives $\delta_{B}\left(t^{-}\right) \chi_{\phi}^{-1}\left(t^{-}\right) \sum_{i} \int_{n \in N^{-}\left(p \mathbb{Z}_{p}\right)} f\left(g_{i} n\right) d \mu_{i} \cdot \operatorname{Vol}\left(\tilde{K}_{\phi}\right)$. On the other hand, using the model of $\pi_{f_{\phi}, p}$ and $\pi_{f_{\phi}^{\vee}, p}$ as the induced representation $\pi\left(\chi_{1, \phi}, \ldots, \chi_{r, \phi}\right)$ and $\pi\left(\chi_{1, \phi}^{-1}, \ldots, \chi_{r, \phi}^{-1}\right)$ of $\mathrm{GL}_{r}\left(\mathbb{Q}_{p}\right)$, we get that

$$
\left\langle\boldsymbol{f}_{\phi}, \pi_{\boldsymbol{f}_{\phi}}^{\vee}\left(t^{-}\right) \boldsymbol{f}_{\phi}^{\vee}\right\rangle=\delta_{B}\left(t^{-}\right) \chi_{\phi}^{-1}\left(t^{-}\right)\left\langle\boldsymbol{f}_{\phi}, \boldsymbol{f}_{\phi}^{\vee}\right\rangle
$$

This proves that the specialization of $\left\langle\boldsymbol{f}, \boldsymbol{f}^{\vee}\right\rangle$ to $\phi$ is $\left\langle\boldsymbol{f}_{\phi}, \boldsymbol{f}_{\phi}^{\vee}\right\rangle \cdot \operatorname{Vol}\left(\tilde{K}_{\phi}\right)^{-1}$.

So, to see the main theorem in the case when $s=0$, instead of applying the Hecke operator $e^{\text {ord }} \cdot 1_{f^{\vee}}$ we pair the pullback of Siegel Eisenstein series (听 $\llbracket \Gamma_{\mathscr{K}} \rrbracket$-valued) with the measure determined by the Hida family $f$ using the above lemma. That is, considering

$$
E_{\mathrm{Kling}}\left(g, z_{\kappa}\right)=\sum_{i} \int_{n \in N^{-}\left(p \mathbb{C}_{F, p}\right)} E_{\mathrm{sieg}}\left(S^{-1} \alpha\left(g, g_{i} n\right) S, z_{\kappa}\right) d \mu_{i},
$$

where the $\left\{d \mu_{i}\right\}_{i}$ are the measures constructed from $\boldsymbol{f}$ as above. In our situation, when restricting to $\mathrm{U}(s, r)$, the level group at $p$ for Eisenstein series is lowertriangular modulo a certain power of $p$ while that for $f$ is upper-triangular modulo a certain power of $p$. The above construction works in the same way. The powers of $\mathrm{CM}$ and $p$-adic periods enter when applying the comparison between the standard basis and the Néron basis for differentials of CM abelian varieties while doing pullback (see [Hsieh 2014, (3.14)]).

5D. Constant terms. We explain part (iii) of the main theorem.

5D1. $p$-adic L-functions for Dirichlet characters. There is an element $\mathscr{L}_{\bar{\tau}^{\prime}}$ in $\Lambda_{\mathscr{L}, O_{L}}$ such that $\phi\left(\mathscr{L}_{\bar{\tau}^{\prime}}\right)=L\left(\bar{\tau}_{\phi}^{\prime}, \kappa_{\phi}-r\right) \cdot \tau_{\phi}^{\prime}\left(p^{-1}\right) p^{\kappa_{\phi}-r} \mathfrak{g}\left(\bar{\tau}_{\phi}^{\prime}\right)^{-1}$ at each arithmetic point $\phi$ in $\mathscr{X}^{\mathrm{pb}}$. For more details see [Skinner and Urban 2014, §3.4.3].

5D2. Archimedean computation. As in [Skinner and Urban 2014], we calculate the Archimedean part of the intertwining operator for Klingen Eisenstein sections and prove the "intertwining operator" part (see Lemma 3.4) of the constant term vanishes. Suppose $\pi$ is associated to the weight $(0, \ldots, 0 ; \kappa, \ldots, \kappa)$; then it is well known that there is a unique (up to scalar) vector $v \in \pi$ such that $k \cdot v=\operatorname{det} \mu(k, i)^{-\kappa}$ for any $k \in K_{\infty}^{+, \prime} v$ (with notations as in Section 3A1). Recall we defined $c(\rho, z)$ in Section 3 A1. 
Lemma 5.10. With assumptions as above,

$$
\begin{aligned}
c(\rho, z)=\pi^{a+2 b+1} \prod_{i=0}^{b-1} & \left(\frac{1}{z+\frac{1}{2} \kappa-\frac{1}{2}-i-a} \frac{1}{z-\frac{1}{2} \kappa+\frac{1}{2}-i}\right) \prod_{i=0}^{a-1} \frac{1}{-1+i-2 z+2 b} \\
& \times \frac{\Gamma(2 z+a) 2^{-1-2 z+2 b}}{\Gamma\left(\frac{1}{2}(a+1)+z+\frac{1}{2} \kappa\right) \Gamma\left(\frac{1}{2}(a+1)+z-\frac{1}{2} \kappa\right)} \operatorname{det}\left(\frac{1}{2} i \zeta\right)^{-2} .
\end{aligned}
$$

Proof. This follows the same way as [Skinner and Urban 2014, Lemma 9.3].

Corollary 5.11. When $\kappa>\frac{3}{2} a+2 b$, or $\kappa \geq 2 b$ and $a=0$, we have $c(\rho, z)=0$ at the point $z=\frac{1}{2}(\kappa-a-2 b-1)$.

In the case when $\kappa$ is sufficiently large, the intertwining operator

$$
A\left(\rho, z_{\kappa}, F\right)=A\left(\rho_{\infty}, z_{\kappa}, F_{\kappa}\right) \otimes A\left(\rho_{f}, z_{\kappa}, F_{f}\right)
$$

and all terms are absolutely convergent. Thus, as a consequence of the above corollary we have $A\left(\rho, z_{\kappa}, F\right)=0$. Therefore the constant term of $E_{\mathrm{Kling}}$ is essentially

$$
\frac{L^{\Sigma}\left(\tilde{\pi}, \bar{\tau}^{c}, z_{\kappa}+1\right)}{\Omega_{\infty}^{2 \kappa \Sigma}\left\langle\tilde{\varphi}^{\text {ord }}, \varphi^{\prime \prime}\right\rangle} \cdot L^{\Sigma}\left(2 z_{\kappa}+1, \bar{\tau}^{\prime} \chi_{\mathscr{K}}^{a+2 b}\right) \varphi
$$

up to a product of normalization factors at local places. Interpolating the calculations in $p$-adic families, part (iii) of Theorem 1.1 follows from the above discussion, Lemma 3.4 and our local descriptions for the $F_{\varphi_{v}}\left(z ; f_{v, \text { sieg }}, g\right)$ in Section 4. (See also the proof of [Skinner and Urban 2014, Theorem 12.11].)

\section{Index of symbols}

$\begin{array}{llllll}A(\rho, z,-) & 1975 & H & 1965 & M_{P} & 1962 \\ B & 1962 & I_{n}(\chi) & 1978 & N_{P} & 1962 \\ \delta(m) & 1975 & J_{n} & 1984 & P & 1961 \\ E_{P} & 1977 & \underline{k} & 1966 & \phi^{\prime} & 2012 \\ E_{R} & 1977 & K & 2005 & \pi^{\vee} & 1975 \\ \tilde{f}^{\dagger} & 1999 & K^{\prime} & 2006 & Q_{n} & 1978 \\ f^{\dagger} & 1999 & K^{\prime \prime} & 2006 & \rho^{\vee} & 1975 \\ f_{v, \text { sieg }} & 1989 & \tilde{K}^{\prime} & 2006 & S^{\prime} & 1985 \\ f^{0} & 2014 & \tilde{K}^{\prime \prime} & 2006 & \theta_{r, s} & 1961 \\ F_{\kappa} & 1976 & K_{\infty}^{+, \prime} & 1974 & { }^{\prime} & 2005 \\ F_{v}^{0} & 2015 & K_{\infty}^{\prime} & 1974 & w_{n} & 1981 \\ F_{\rho_{v}} & 1976 & K_{n, v} & 1978 & X^{+} & 1965 \\ G & 1961 & l_{k} & 1966 & \langle X, X\rangle_{h} & 1983 \\ \gamma & 2005 & M(z ;-) & 1979 & & \end{array}$




\title{
Appendix: Boundary strata of connected components in positive characteristics
}

\author{
by Kai-Wen Lan
}

Under the assumption that the PEL datum involves no factor of type D and that the integral model has good reduction, we show that all boundary strata of the toroidal or minimal compactifications of the integral model (constructed in earlier works of the author) have nonempty pullbacks to connected components of geometric fibers, even in positive characteristics.

A.1. Introduction. Toroidal and minimal compactifications of Shimura varieties and their integral models have played important roles in the study of arithmetic properties of cohomological automorphic representations. While all known models of them are equipped with natural stratifications, they often suffer from some imprecisions or redundancies due to their constructions. The situation is especially subtle in positive or mixed characteristics, or when we need purely algebraic constructions even in characteristic zero (for example, when we study the degeneration of abelian varieties), where the constructions are much less direct than algebraizing complex manifolds created by unions of explicit double coset spaces.

For example, integral models of Shimura varieties defined by moduli problems of PEL structures suffer from the so-called failure of Hasse's principle, because there is no known way to tell the difference between two moduli problems associated with algebraic groups which are everywhere locally isomorphic to each other. Similarly, when their toroidal and minimal compactifications are constructed using the theory of degeneration, the data for describing them are also local in nature. Unlike in the complex analytic construction, one cannot just express all the boundary points as the disjoint unions of some double coset spaces labeled by certain standard maximal (rational) parabolic subgroups. (Even the nonemptiness of the whole boundaries in positive characteristics was not straightforward - see the introduction to [Lan 2011].) As we shall see, in Example A.7.2, when factors of type D are allowed, it is unrealistic to expect that the boundary stratifications in the algebraic and complex analytic constructions match with each other.

Our goal here is a simple-minded one - to show that the strata of good reduction integral models of toroidal and minimal compactifications constructed as in [Lan 2013a] have nonempty pullbacks to each connected component of each geometric fiber, under the assumption that the data defining them involve no factors of type $\mathrm{D}$ (in a sense we will make precise). We will also answer the analogous question for the integral models constructed by normalization in [Lan 2014], allowing arbitrarily deep levels and ramifications (that is, bad reductions in general).

This goal is motivated by the study of $p$-adic families of Eisenstein series, 
for which it is crucial to know that the strata on connected components of the characteristic- $p$ fibers are all nonempty. For example, this is useful for the consideration of algebraic Fourier-Jacobi expansions. We expect it to play foundational roles in other applications of a similar nature.

A.2. Main result. We shall formulate our results in the notation system of [Lan 2013a] — henceforth abbreviated [KWL] — which we shall briefly review. (We shall follow [KWL, Notation and conventions, pp. xxi-xxiii] unless otherwise specified. While for practical reasons we cannot explain everything we need from there, we recommend the reader to make use of the reasonably detailed index and table of contents there when looking for the numerous definitions.)

Let $\left(\mathcal{O}, \star, L,\langle\cdot, \cdot\rangle, h_{0}\right)$ be an integral PEL datum, where $\mathbb{O}, \star$, and $\left(L,\langle\cdot, \cdot\rangle, h_{0}\right)$ are as in [KWL, Definition 1.2.1.3], satisfying [KWL, Condition 1.4.3.10], which defines a group functor $\mathrm{G}$ over $\mathbb{Z}$ as in [KWL, Definition 1.2.1.6], and the reflex field $F_{0}$ (as a subfield of $\mathbb{C}$ ), as in [KWL, Definition 1.2.5.4], with ring of integers $0_{F_{0}}$. Let $p$ be any good prime, as in [KWL, Definition 1.4.1.1]. Let $\mathscr{H}^{p}$ be any open compact subgroup of $\mathrm{G}\left(\hat{\mathbb{Z}}^{p}\right)$ that is neat, as in [KWL, Definition 1.4.1.8]. Then we have a moduli problem $\mathrm{M}_{\mathscr{p}} p$ over $\mathrm{S}_{0}=\operatorname{Spec}\left(\mathrm{O}_{F_{0},(p)}\right)$, as in [KWL, Definition 1.4.1.4], which is representable by a scheme that is quasiprojective and smooth over $\mathrm{S}_{0}$, by [KWL, Theorem 1.4.1.11 and Corollary 7.2.3.10]. By [KWL, Theorem 7.2.4.1 and Proposition 7.2.4.3], we have the minimal compactification $\mathrm{M}_{\mathscr{H}^{p}}^{\min }$ of $\mathrm{M}_{\mathscr{H}^{p}}$, which is a scheme that is projective and flat over $\mathrm{S}_{0}$, with geometrically normal fibers. Moreover, for each compatible collection $\Sigma^{p}$ of cone decompositions for $\mathrm{M}_{\mathscr{H}^{p}}$, as in [KWL, Definition 6.3.3.4], we also have the toroidal compactification $\mathrm{M}_{\mathscr{H}^{p}, \Sigma^{p}}^{\text {tor }}$ of $\mathrm{M}_{\mathscr{H}^{p}}$, which is an algebraic space that is proper and smooth over $\mathrm{S}_{0}$, by [KWL, Theorem 6.4.1.1], and which is representable by a scheme projective over $\mathrm{M}_{0}$ when $\Sigma^{p}$ is projective, as in [KWL, Definition 7.3.1.3], by [KWL, Theorem 7.3.3.4]. Any such $\mathrm{M}_{\mathscr{H}^{p}, \Sigma^{p}}^{\text {tor }}$ admits a canonical surjection $\oint_{\mathscr{H}^{p}}: \mathrm{M}_{\mathscr{H}^{p}, \Sigma^{p}}^{\text {tor }} \rightarrow \mathrm{M}_{\mathscr{H}^{p}}^{\min }$, which is constructed by Stein factorization as in [KWL, Section 7.2.3], whose fibers are all geometrically connected. (The superscript " $p$ " indicates that the objects are defined using level structures "away from $p$ ". We will also encounter their variants without the superscript " $p$ ", which also involve level structures "at $p$ ".)

By [KWL, Theorem 7.2.4.1(4)], there is a stratification of $\mathrm{M}_{\mathscr{H} e p}^{\min }$ by locally closed subschemes $Z_{\left[\left(\Phi_{\mathscr{H}}, \delta_{\mathscr{C}} p\right)\right]}$, where $\left[\left(\Phi_{\mathscr{H}}, \delta_{\mathscr{H} P}\right)\right]$ runs through the (finite) set of cusp labels for M⿻丷 (see [KWL, Definition 5.4.2.4]). The open dense subscheme $\mathrm{M}_{\mathscr{H} P}$ is the stratum labeled by $[(0,0)]$; we call all the other strata the

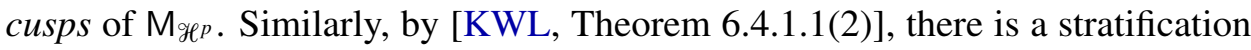
of $\mathrm{M}_{\mathscr{H} \mathcal{H}^{p}, \Sigma^{p}}^{\text {tor }}$ by locally closed subschemes $\mathrm{Z}_{\left[\left(\Phi_{\mathscr{F}} p, \delta_{\mathscr{H}}, \sigma^{p}\right)\right]}$, where $\left[\left(\Phi_{\mathscr{H}^{p}}, \delta_{\mathscr{H}^{p}}, \sigma^{p}\right)\right]$ runs through equivalence classes, as in [KWL, Definition 6.2.6.1], with $\sigma^{p} \subset \boldsymbol{P}_{\Phi_{\mathscr{F}} p}^{+}$ and $\sigma^{p} \in \Sigma_{\Phi_{\mathscr{F}} p} \in \Sigma^{p}$. By [KWL, Theorem 7.2.4.1(5)], the surjection $\oint_{\mathscr{H e}}$ induces 
a surjection from the $\left[\left(\Phi_{\mathscr{H}}, \delta_{\mathscr{H}^{p}}, \sigma^{p}\right)\right]$-stratum $\mathrm{Z}_{\left[\left(\Phi_{\mathscr{H} P}, \delta_{\mathscr{F} P}, \sigma^{p}\right)\right]}$ of $\mathrm{M}_{\mathscr{H}^{p}, \Sigma^{p}}^{\text {tor }}$ to the $\left[\left(\Phi_{\mathscr{H} p}, \delta_{\mathscr{H}^{p}}\right)\right]$-stratum $\mathrm{Z}_{\left[\left(\Phi_{\mathscr{H} P}, \delta_{\mathscr{H} P}\right)\right]}$ of $\mathrm{M}_{\mathscr{H} \mathcal{P}}^{\min }$.

Let $s \rightarrow \mathrm{S}_{0}$ be any geometric point with residue field $k(s)$, and let $U$ be any connected component of the fiber $\mathrm{M}_{\mathscr{H}^{p}} \times{ }_{\mathrm{S}_{0}} s$. Since $\mathrm{M}_{\mathscr{H}^{p}}^{\min } \rightarrow \mathrm{S}_{0}$ is proper and has geometrically normal fibers, the closure $U^{\min }$ of $U$ in $\mathrm{M}_{\mathscr{H}}^{\min } \times \mathrm{s}_{0} s$ is a connected component of $\mathrm{M}_{\mathscr{H}^{p}}^{\min } \times \mathrm{S}_{0} s$. Similarly, since $\mathrm{M}_{\mathscr{H}^{p}}^{\text {tor }}, \Sigma^{p} \rightarrow \mathrm{S}_{0}$ is proper and smooth, the closure $U^{\text {tor }}$ of $U$ in $\mathrm{M}_{\mathscr{H}^{p}, \Sigma^{p}}^{\text {tor }} \times \mathrm{s}_{0} s$ is a connected component of $\mathrm{M}_{\mathscr{H}^{p}, \Sigma^{p}}^{\text {tor }} \times \mathrm{S}_{0} s$. (In these cases the connected components are also the irreducible components of the ambient spaces.)

The stratifications of $\mathrm{M}_{\mathscr{F}^{p}}^{\min }$ and $\mathrm{M}_{\mathscr{F}^{p}, \Sigma^{p}}^{\text {tor }}$ induce stratifications of $U^{\mathrm{min}}$ and $U^{\text {tor }}$, respectively, by pullback. We shall denote the pullback of $Z_{\left[\left(\Phi_{\mathscr{F}}, \delta_{\mathscr{H}} p\right)\right]}$ to $U^{\text {min }}$ by $U_{\left[\left(\Phi_{\mathscr{H}}, \delta_{\mathscr{H}} p\right)\right]}$ and call it the $\left[\left(\Phi_{\mathscr{H}}, \delta_{\mathscr{H}^{p}}\right)\right]$-stratum of $U^{\mathrm{min}}$. Similarly, we shall denote the pullback of $Z_{\left[\left(\Phi_{\mathscr{F}}, \delta_{\mathscr{F}}, \sigma^{p}\right)\right]}$ to $U^{\text {tor }}$ by $U_{\left[\left(\Phi_{\mathscr{K} P}, \delta_{\mathscr{F}}, \sigma^{p}\right)\right]}$, and call it the $\left[\left(\Phi_{\mathscr{H}^{p}}, \delta_{\mathscr{H}^{p}}, \sigma^{p}\right)\right]$-stratum of $U^{\text {tor }}$. By construction, the surjection $\oint_{\mathscr{H} P}$ induces a surjection $U^{\text {tor }} \rightarrow U^{\text {min }}$, which maps the $\left[\left(\Phi_{\mathscr{H}^{p}}, \delta_{\mathscr{H}^{p}}, \sigma^{p}\right)\right]$-stratum $U_{\left[\left(\Phi_{\mathscr{F}}, \delta_{\mathscr{F}}, \sigma^{p}\right)\right]}$ of $U^{\text {tor }}$ surjectively onto the $\left[\left(\Phi_{\mathscr{H}}, \delta_{\mathscr{H} P}\right)\right]$-stratum $U_{\left[\left(\Phi_{\mathscr{H}}, \delta_{\mathscr{H}} p\right)\right]}$ of $U^{\mathrm{min}}$. It is natural to ask whether a particular stratum of $U^{\mathrm{min}}$ or $U^{\text {tor }}$ is nonempty.

From now on, we shall assume the following:

Assumption A.2.1. The semisimple algebra $\mathbb{O} \otimes_{\mathbb{Z}} \mathbb{Q}$ over $\mathbb{Q}$ involves no factor of type D (in the sense of [KWL, Definition 1.2.1.15]).

Our main result is the following:

Theorem A.2.2. With the setting as above, all strata of $U^{\min }$ are nonempty.

An immediate consequence is the following:

Corollary A.2.3. With the setting as above, all strata of $U^{\text {tor }}$ are nonempty.

Proof. Since the canonical morphism $U_{\left[\left(\Phi_{\mathscr{F}} p, \delta_{\mathscr{H} P}, \sigma^{p}\right)\right]} \rightarrow U_{\left[\left(\Phi_{\mathscr{H} P}, \delta_{\mathscr{H} P}\right)\right]}$ is surjective for each equivalence class $\left[\left(\Phi_{\mathscr{H}^{p}}, \delta_{\mathscr{H}^{p}}, \sigma^{p}\right)\right]$ with underlying cusp label $\left[\left(\Phi_{\mathscr{H}^{p}}, \delta_{\mathscr{H}^{p}}\right)\right]$ as above, the nonemptiness of $U_{\left[\left(\Phi_{\mathscr{F}}, \delta_{\mathscr{F} P}\right)\right]}$ implies that of $U_{\left[\left(\Phi_{\mathscr{F} P}, \delta_{\mathscr{F}}, \sigma^{p}\right)\right]}$.

Remark A.2.4. Each stratum $Z_{\left[\left(\Phi_{\mathscr{F}}, Z_{\mathscr{F}} p\right)\right]}\left(\operatorname{resp} . Z_{\left[\left(\Phi_{\mathscr{F}}, Z_{\mathscr{F}} p, \sigma^{p}\right)\right]}\right)$ is nonempty by [KWL, Theorem 7.2.4.1(4)-(5), Corollary 6.4.1.2, and the explanation of the existence of complex points as in Remark 1.4.3.14]. The question is whether its pullback to $U^{\text {min }}$ (resp. $U^{\text {tor }}$ ) is still nonempty for every $U$ as above.

Remark A.2.5. It easily follows from Theorem A.2.2 and Corollary A.2.3 that their analogues are also true when the geometric point $s \rightarrow \mathrm{S}_{0}$ is replaced with morphisms from general schemes, although we shall omit their statements. In particular, we can talk about connected components of fibers rather than geometric fibers.

The proof of Theorem A.2.2 will be carried out in Sections A.3, A.4, and A.5. In Sections A.5 and A.6, we will also state and prove analogues of Theorem A.2.2 in 
zero and arbitrarily ramified characteristics, respectively (see Theorems A.5.1 and A.6.1). We will give some examples in Section A.7, including one (see Example A.7.2) showing that we cannot expect Theorem A.2.2 to be true without the requirement (in Assumption A.2.1) that $O \otimes_{\mathbb{Z}} \mathbb{Q}$ involves no factor of type $\mathrm{D}$.

A.3. Reduction to the case of characteristic zero. The goal of this section is to prove the following:

Proposition A.3.1. Suppose Theorem A.2.2 is true when $\operatorname{char}(k(s))=0$. Then it is also true when $\operatorname{char}(k(s))=p>0$.

Remark A.3.2. Proposition A.3.1 holds regardless of Assumption A.2.1.

Remark A.3.3. It might seem that everything in characteristic zero is well known and straightforward. But Proposition A.3.1, which is insensitive to the crucial Assumption A.2.1, shows that the key difficulty is in fact in characteristic zero.

By [KWL, Theorem 7.2.4.1(4)], each $Z_{\left[\left(\Phi_{\mathscr{F}}, \delta_{\mathscr{H} P}\right)\right]}$ is isomorphic to a boundary moduli problem $\mathrm{M}_{\mathscr{H} P}^{Z_{\mathscr{F}} p}$ defined in the same way as $\mathrm{M}_{\mathscr{H}} p$ (but with certain integral PEL datum associated with $Z_{\mathscr{H}} p$ ). Then it makes sense to consider the minimal compactification $Z_{\left[\left(\Phi_{\mathscr{F}}, \delta_{\mathscr{H}} p\right)\right]}^{\min }$ of $Z_{\left[\left(\Phi_{\mathscr{F}}, \delta_{\mathscr{F} P}\right)\right]}$, which is proper flat and has geometrically normal fibers over $M_{\mathscr{H}}$, as in [KWL, Theorem 7.2.4.1 and Proposition 7.2.4.3]. (So the connected components of the geometric fibers of $Z_{\left[\left(\Phi_{\mathscr{H}}, \delta_{\mathscr{H} P}\right)\right]} \rightarrow \mathrm{S}_{0}$ are closures of those of $Z_{\left[\left(\Phi_{\mathscr{F}}, \delta_{\mathscr{F}} p\right)\right]} \rightarrow \mathrm{S}_{0}$.) By considering the Stein factorizations of the structural morphisms $Z_{\left[\left(\Phi_{\mathscr{F}}, \delta_{\mathscr{F}} p\right)\right]}^{\min } \rightarrow \mathrm{S}_{0}$ (see [EGA III 1 1961, Corollaire (4.3.3) and Remarque (4.3.4), pp. 131-132]), we obtain the following:

Lemma A.3.4 (cf. [KWL, Corollary 6.4.1.2] and [Deligne and Mumford 1969, Theorem 4.17]). Suppose $\operatorname{char}(k(s))=p>0$. Then there exists some discrete valuation ring $R$ that is flat over $O_{F_{0}},(p)$, with fraction field $K$ and residue field $k(s)$, the latter lifting the structural homomorphism $\mathrm{O}_{F_{0},(p)} \rightarrow k(s)$ such that, for each cusp label $\left[\left(\Phi_{\mathscr{H}}, \delta_{\mathscr{H} p}\right)\right]$ and each connected component $V$ of $\mathrm{Z}_{\left[\left(\Phi_{\mathscr{F}}, \delta_{\mathscr{F} p}\right)\right]} \otimes_{\mathcal{O}_{F_{0}},(p)} R$, the induced flat morphism $V \rightarrow \operatorname{Spec}(R)$ has connected special fiber over $\operatorname{Spec}(k(s))$.

Proof of Proposition A.3.1. Let $R$ be as in Lemma A.3.4. Let $\tilde{U}$ denote the connected component of $\mathrm{M}_{\mathcal{H} p} \otimes_{\mathcal{O}_{F_{0},(p)}} R=\mathrm{Z}_{[(0,0)]} \otimes_{\mathcal{O}_{F_{0},(p)}} R$ such that $\tilde{U} \otimes_{R} k(s)=U$ as subsets of $\mathrm{M}_{\mathscr{H}^{p}} \otimes_{\mathcal{O}_{F_{0}},(p)} k(s)=\mathrm{M}_{\mathscr{H}^{p}} \times \mathrm{S}_{0} s$, and let $\tilde{U}^{\mathrm{min}}$ denote its closure in $\mathrm{M}_{\mathscr{H} \mathcal{P}}^{\min } \otimes_{\mathcal{O}_{F_{0},(p)}} R$, which is a connected component of $\mathrm{M}_{\mathscr{H} p}^{\min } \otimes_{\mathcal{O}_{F_{0}},(p)} R$ because $\mathrm{M}_{\mathscr{H}^{p}}^{\min } \otimes_{\mathcal{O}_{F_{0}},(p)} R$ is normal, by [KWL, Proposition 7.2.4.3(4)]. For each cusp label $\left[\left(\Phi_{\mathscr{H}}, \delta_{\mathscr{H}} p\right)\right]$, let $\tilde{U}_{\left[\left(\Phi_{\mathscr{H}} p, \delta_{\mathscr{H}} p\right)\right]}$ denote the pullback of $\mathrm{Z}_{\left[\left(\Phi_{\mathscr{K} P}, \delta_{\mathscr{Y} P}\right)\right]}$ to $\tilde{U}^{\mathrm{min}}$. Then $\tilde{U}_{\left[\left(\Phi_{\mathscr{F}} p, \delta_{\mathscr{H}} p\right)\right]}$ is an open and closed subscheme of $\mathrm{Z}_{\left[\left(\Phi_{\mathscr{H} P}, \delta_{\mathscr{F}} p\right)\right]} \otimes_{\mathcal{O}_{F_{0}},(p)} R$ such that $\tilde{U}_{\left[\left(\Phi_{\mathscr{F}}, \delta_{\mathscr{H}} p\right)\right]} \otimes_{R} k(s)=U_{\left[\left(\Phi_{\mathscr{F}}, \delta_{\mathscr{F}} p\right)\right]}$ as subsets of $\mathrm{M}_{\mathscr{H} p}^{\min } \otimes_{\mathcal{O}_{F_{0},(p)}} k(s)$. By Lemma A.3.4, it suffices to show that $\tilde{U}_{\left[\left(\tilde{U}_{\mathscr{Y}}, \delta_{\mathscr{F}} p\right)\right]} \otimes_{R} \bar{K} \neq \varnothing$ for some algebraic closure $\bar{K}$ of $K$. Also by Lemma A.3.4, $\tilde{U} \otimes_{R} \bar{K} \neq \varnothing$, and so $\tilde{U}^{\text {min }} \otimes_{R} \bar{K}$ contains at least one 
connected component of $\mathrm{M}_{\mathscr{H} P}^{\min } \otimes_{\mathcal{O}_{F_{0},(p)}} \bar{K}$. Thus, $\tilde{U}_{\left[\left(\Phi_{\mathscr{F}}, \delta_{\mathscr{C} P}\right)\right]} \otimes_{R} \bar{K} \neq \varnothing$ under the assumption of the proposition, as desired.

A.4. Comparison of cusp labels. Let $\mathscr{H}_{p}:=\mathrm{G}\left(\mathbb{Z}_{p}\right)$ and $\mathscr{H}:=\mathscr{H}^{p} \mathscr{H}_{p}$, the latter being a neat open compact subgroup of $\mathrm{G}(\hat{\mathbb{Z}})$. By the same references to [KWL] as in Section A.2, we have the moduli problem $\mathrm{M}_{\mathscr{H}}$ and its minimal compactification $\mathrm{M}_{\mathscr{H}}^{\min }$ over $\mathrm{S}_{0, \mathbb{Q}}:=\mathrm{S}_{0} \otimes_{\mathbb{Z}} \mathbb{Q} \cong \operatorname{Spec}\left(F_{0}\right)$. For each compatible collection $\Sigma^{\prime}$ of cone decompositions for $\mathrm{M}_{\mathscr{H}}$, we also have a toroidal compactification $\mathrm{M}_{\mathscr{H}, \Sigma^{\prime}}^{\text {tor }}$, together with a canonical morphism $\oint_{\mathscr{H}}: \mathrm{M}_{\mathscr{H}, \Sigma^{\prime}}^{\text {tor }} \rightarrow \mathrm{M}_{\mathscr{H}}^{\min }$, over $\mathrm{S}_{0, \mathbb{Q}}$. (Here $\Sigma^{\prime}$ does not have to be related to the $\Sigma^{p}$ above.)

Each cusp label $\left[\left(\mathrm{Z}_{\mathscr{H}}, \Phi_{\mathscr{H}}, \delta_{\mathscr{H}}\right)\right]$ for $\mathrm{M}_{\mathscr{H}}\left(\right.$ where $\mathrm{Z}_{\mathscr{H}}$ has been suppressed in the notation for simplicity) can be described as an equivalence class of the $\mathscr{H}$-orbit $\left(\mathrm{Z}_{\mathscr{H}}, \Phi_{\mathscr{H}}, \delta_{\mathscr{H}}\right)$ of some triple $(\mathrm{Z}, \Phi, \delta)$, where:

(1) $\mathrm{Z}=\left\{\mathrm{Z}_{-i}\right\}_{i \in \mathbb{Z}}$ is an admissible filtration on $L \otimes_{\mathbb{Z}} \hat{\mathbb{Z}}$ that is fully symplectic, as in [KWL, Definition 5.2.7.1]. In particular, $Z_{-i}=\left(Z_{-i} \otimes_{\mathbb{Z}} \mathbb{Q}\right) \cap\left(L \otimes_{\mathbb{Z}} \hat{\mathbb{Z}}\right)$, the symplectic filtration $Z \otimes_{\mathbb{Z}} \mathbb{Q}$ on $L \otimes_{\mathbb{Z}} \mathbb{A}^{\infty}$ extends to a symplectic filtration $Z_{\mathbb{A}}$ on $Z \otimes_{\mathbb{Z}} A$, and each graded piece of $Z$ or $Z \otimes_{\mathbb{Z}} \mathbb{Q}$ is integrable, as in [KWL, Definition 1.2.1.23], that is, it is the base extension of some 0 -lattice.

(2) $\Phi=\left(X, Y, \phi, \varphi_{-2}, \varphi_{0}\right)$ is a torus argument, as in [KWL, Definition 5.4.1.3], where $\phi: Y \hookrightarrow X$ is an embedding of $\mathbb{C}$-lattices with finite cokernel, and where $\varphi_{-2}: \mathrm{Gr}_{-2}^{Z} \stackrel{\sim}{\rightarrow} \operatorname{Hom}_{\hat{\mathbb{Z}}}\left(X \otimes_{\mathbb{Z}} \hat{\mathbb{Z}}, \hat{\mathbb{Z}}(1)\right)$ and $\varphi_{0}: \mathrm{Gr}_{0}^{Z} \stackrel{\sim}{\rightarrow} Y \otimes_{\mathbb{Z}} \hat{\mathbb{Z}}$ are isomorphisms matching the pairing $\langle\cdot, \cdot\rangle_{20}: \mathrm{Gr}_{-2}^{Z} \times \mathrm{Gr}_{0}^{Z} \rightarrow \hat{\mathbb{Z}}(1)$ induced by $\langle\cdot, \cdot\rangle$ with the pairing $\langle\cdot, \cdot\rangle_{\phi}: \operatorname{Hom}_{\hat{\mathbb{Z}}}\left(X \otimes_{\mathbb{Z}} \hat{\mathbb{Z}}, \hat{\mathbb{Z}}(1)\right) \times\left(Y \otimes_{\mathbb{Z}} \hat{\mathbb{Z}}\right) \rightarrow \hat{\mathbb{Z}}(1)$ induced by $\phi$.

(3) $\delta: \mathrm{Gr}^{\mathrm{Z}} \stackrel{\sim}{\longrightarrow} L$ is an $\mathrm{O}$-equivariant splitting of the filtration $\mathrm{Z}$.

(4) Two triples $\left(Z_{\mathscr{H}}, \Phi_{\mathscr{H}}, \delta_{\mathscr{H}}\right)$ and $\left(\mathrm{Z}_{\mathscr{H}}^{\prime}, \Phi_{\mathscr{H}}^{\prime}, \delta_{\mathscr{H}}^{\prime}\right)$ are equivalent (as in [KWL, Definition 5.4.2.2]) if $\mathrm{Z}_{\mathscr{H}}=\mathrm{Z}_{\mathscr{H}}^{\prime}$ and there exists a pair of isomorphisms, $\gamma_{X}: X^{\prime} \stackrel{\sim}{\longrightarrow} X$ and $\gamma_{Y}: Y \stackrel{\sim}{\longrightarrow} Y^{\prime}$, matching $\Phi_{\mathscr{H}}$ with $\Phi_{\mathscr{H}}^{\prime}$.

Since $\mathscr{H}=\mathscr{H}^{p} \mathscr{H}_{p}$, it makes sense to consider the $p$-part of $\left(\mathrm{Z}_{\mathscr{H}}, \Phi_{\mathscr{H}}, \delta_{\mathscr{H}}\right)$, which is the $\mathcal{H}_{p}$-orbit of some triple $\left(\mathrm{Z}_{\mathbb{Z}_{p}},\left(\varphi_{-2, \mathbb{Z}_{p}}, \varphi_{0, \mathbb{Z}_{p}}\right), \delta_{\mathbb{Z}_{p}}\right)$, where:

(1) $Z_{\mathbb{Z}_{p}}=\left\{Z_{\mathbb{Z}_{p},-i}\right\}_{i \in \mathbb{Z}}$ is a symplectic admissible filtration on $L \otimes_{\mathbb{Z}} \mathbb{Z}_{p}$, which determines and is determined by a symplectic admissible filtration $\mathrm{Z}_{\mathbb{Q}_{p}}=\left\{Z_{\mathbb{Q}_{p},-i}\right\}_{i \in \mathbb{Z}}$ of $L \otimes_{\mathbb{Z}} \mathbb{Q}_{p}$ by $Z_{\mathbb{Q}_{p},-i}=\mathrm{Z}_{\mathbb{Z}_{p},-i} \otimes_{\mathbb{Z}} \mathbb{Q}$ and $\mathrm{Z}_{\mathbb{Z}_{p},-i}=\mathrm{Z}_{\mathbb{Q}_{p},-i} \cap\left(L \otimes_{\mathbb{Z}} \mathbb{Z}_{p}\right)$ for all $i \in \mathbb{Z}$. (2) $\varphi_{-2, \mathbb{Z}_{p}}: \mathrm{Gr}_{-2}^{\mathrm{Z}_{\mathbb{Z}_{p}}} \stackrel{\sim}{\rightarrow} \operatorname{Hom}_{\mathbb{Z}_{p}}\left(X \otimes_{\mathbb{Z}} \mathbb{Z}_{p}, \mathbb{Z}_{p}(1)\right)$ and $\varphi_{0}: \mathrm{Gr}_{0}^{\mathbf{Z}_{\mathbb{Z}_{p}}} \stackrel{\sim}{\rightarrow} Y \otimes_{\mathbb{Z}} \mathbb{Z}_{p}$ are isomorphisms matching the pairing $\langle\cdot, \cdot\rangle_{20, \mathbb{Z}_{p}}: \mathrm{Gr}_{-2}^{\mathrm{Z}_{p}} \times \mathrm{Gr}_{0} \mathrm{Z}_{\mathbb{Z}_{p}} \rightarrow \mathbb{Z}_{p}(1)$ induced by $\langle\cdot, \cdot\rangle$ with the pairing $\langle\cdot, \cdot\rangle_{\phi, \mathbb{Z}_{p}}: \operatorname{Hom}_{\mathbb{Z}_{p}}\left(X \otimes_{\mathbb{Z}} \mathbb{Z}_{p}, \mathbb{Z}_{p}(1)\right) \times\left(Y \otimes_{\mathbb{Z}} \mathbb{Z}_{p}\right) \rightarrow \mathbb{Z}_{p}(1)$ induced by $\phi$.

(3) $\delta_{\mathbb{Z}_{p}}: \mathrm{Gr}^{\mathrm{Z}_{\mathbb{Z}_{p}}} \stackrel{\sim}{\longrightarrow} L \otimes_{\mathbb{Z}} \mathbb{Z}_{p}$ is a splitting of the filtration $\mathbb{Z}_{\mathbb{Z}_{p}}$. 
By forgetting its $p$-part, each representative $\left(Z_{\mathscr{H}}, \Phi_{\mathscr{H}}, \delta_{\mathscr{H}}\right)$ for $\mathrm{M}_{\mathscr{H}}$ induces a representative ( $\mathrm{Z}_{\mathscr{H}}, \Phi_{\mathscr{H}}, \delta_{\mathscr{H} \rho}$ ) for $\mathrm{M}_{\mathscr{H}} p$, and this assignment is compatible with the formation of equivalence classes. Therefore, we have well-defined assignments

$$
\left(\mathrm{Z}_{\mathscr{H}}, \Phi_{\mathscr{H}}, \delta_{\mathscr{H}}\right) \mapsto\left(\mathrm{Z}_{\mathscr{H}}, \Phi_{\mathscr{H}}, \delta_{\mathscr{H} P}\right)
$$

and

$$
\left[\left(\mathrm{Z}_{\mathscr{H}}, \Phi_{\mathscr{H}}, \delta_{\mathscr{H}}\right)\right] \mapsto\left[\left(\mathrm{Z}_{\mathscr{H}}, \Phi_{\mathscr{H} p}, \delta_{\mathscr{H} p}\right)\right]
$$

By construction, these assignments are compatible with surjections on both their sides (see [KWL, Definition 5.4.2.12]). We would like to show that they are both bijective.

Lemma A.4.3. Let $k$ be any field over $\mathbb{Z}_{(p)}$. Consider the assignment to each flag $W$ of totally isotropic $\mathbb{O} \otimes_{\mathbb{Z}} k$-submodules of $L \otimes_{\mathbb{Z}} k$ (with respect to $\langle\cdot, \cdot\rangle \otimes_{\mathbb{Z}} k$ ) its stabilizer subgroup $\mathrm{P}_{W}$ in $\mathrm{G} \otimes_{\mathbb{Z}} k$. Then each such $\mathrm{P}_{W}$ is a parabolic subgroup of $\mathrm{G} \otimes_{\mathbb{Z}} k$ and the assignment is bijective. Moreover, given any minimal parabolic subgroup $\mathrm{P}_{W_{0}}$ of $\mathrm{G} \otimes_{\mathbb{Z}} k$, which is the stabilizer of some maximal flag $W_{0}$ of totally isotropic $0 \otimes_{\mathbb{Z}} k$-submodules of $L \otimes_{\mathbb{Z}} k$, every parabolic subgroup of $\mathrm{G} \otimes_{\mathbb{Z}} k$ is conjugate under the action of $\mathrm{G}(k)$ to some parabolic subgroup of $\mathrm{G} \otimes_{\mathbb{Z}} k$ containing $\mathrm{P}_{W_{0}}$, which is the stabilizer of some subflag of $W_{0}$.

Although the assertions in this lemma are well known, we provide a proof because we cannot find a convenient reference in the literature in the generality we need.

Proof. Let $k^{\mathrm{sep}}$ be a separable closure of $k$. Since the characteristic of $k$ is either 0 or $p$, the latter being a good prime by assumption, it follows from [KWL, Proposition 1.2.3.11] that each of the simple factors of the adjoint quotient of $\mathrm{G} \otimes_{\mathbb{Z}} k^{\mathrm{sep}}$ is isomorphic to one of the groups of standard type listed in the proof of [KWL, Proposition 1.2.3.11]. Then we can make an explicit choice of a Borel subgroup B of $\mathrm{G} \otimes_{\mathbb{Z}} k^{\text {sep }}$ stabilizing a flag of totally isotropic submodules, with a maximal torus $\mathrm{T}$ of $\mathrm{G} \otimes_{\mathbb{Z}} k^{\text {sep }}$ contained in $\mathrm{B}$ which is isomorphic to the group of automorphisms of the graded pieces of this flag. By [Springer 1998, Theorem 6.2.7 and Theorem 8.4.3(iv)], since all parabolic subgroups of $\mathrm{G} \otimes_{\mathbb{Z}} k^{\mathrm{sep}}$ are conjugate to one containing $\mathrm{B}$, the parabolic subgroups of $\mathrm{G} \otimes_{\mathbb{Z}} k^{\text {sep }}$ are exactly the stabilizers of flags of totally isotropic $0 \otimes_{\mathbb{Z}} k^{\text {sep }}$-submodules of $L \otimes_{\mathbb{Z}} k^{\text {sep }}$. Then the analogous assertion over $k$ follows, because the assignment of maximal parabolic subgroups of $G \otimes_{\mathbb{Z}} k^{\text {sep }}$ is compatible with the actions of $\operatorname{Gal}\left(k^{\text {sep }} / k\right)$ on the set of flags of totally isotropic submodules of $L \otimes_{\mathbb{Z}} k^{\text {sep }}$ and on the set of parabolic subgroups of $\mathrm{G} \otimes_{\mathbb{Z}} k^{\text {sep }}$. The last assertion of the lemma follows from [Springer 1998, Theorem 15.1.2(ii) and Theorem 15.4.6(i)].

Lemma A.4.4. The assignment

$$
\mathrm{Z}_{\mathscr{H}} \mapsto \mathrm{Z}_{\mathscr{H}_{p}}
$$


is bijective.

Proof. Let $Z_{\mathbb{Z}_{p}}=\left\{Z_{\mathbb{Z}_{p},-i}\right\}_{i \in \mathbb{Z}}$ be a symplectic admissible filtration on $L \otimes_{\mathbb{Z}} \mathbb{Z}_{p}$ as above, which determines and is determined by a symplectic filtration $\mathrm{Z}_{\mathbb{Q}_{p}}=$ $\left\{Z_{\mathbb{Q}_{p},-i}\right\}_{i \in \mathbb{Z}}$ on $L \otimes_{\mathbb{Z}} \mathbb{Q}_{p}$. By Lemma A.4.3, the action of $\mathrm{G}\left(\mathbb{Q}_{p}\right)$ on the set of such filtrations $Z_{\mathbb{Q}_{p}}$ is transitive, because the $\mathrm{C}$-multirank (see [KWL, Definition 1.2.1.25]) of the bottom piece $Z_{\mathbb{Q}_{p},-2}$ of any such $Z_{\mathbb{Q}_{p}}$ is determined by the existence of some isomorphism

$$
\varphi_{-2, \mathbb{Z}_{p}}: \mathrm{Gr}_{-2}^{\mathrm{Z}_{\mathbb{Z}_{p}}} \stackrel{\sim}{\longrightarrow} \operatorname{Hom}_{\mathbb{Z}_{p}}\left(X \otimes_{\mathbb{Z}} \mathbb{Z}_{p}, \mathbb{Z}_{p}(1)\right) .
$$

Let $\mathrm{P}$ denote the parabolic subgroup of $\mathrm{G} \otimes_{\mathbb{Z}} \mathbb{Q}_{p}$ stabilizing any such $\mathrm{Z}_{\mathbb{Q}_{p}}$ (see Lemma A.4.3). Since $p$ is a good prime by assumption, the pairing $\langle\cdot, \cdot\rangle \otimes_{\mathbb{Z}} \mathbb{Z}_{p}$ is self-dual, and hence $G\left(\mathbb{Z}_{p}\right)$ is a maximal open compact subgroup of $G\left(\mathbb{Q}_{p}\right)$, by [Bruhat and Tits 1972, Corollary 3.3.2]. Since $\mathrm{G} \otimes_{\mathbb{Z}} \mathbb{Q}_{p}$ is connected under Assumption A.2.1 (because the kernel of the similitude character of $G \otimes_{\mathbb{Z}} \mathbb{Q}_{p}$ factorizes over an algebraic closure of $\mathbb{Q}_{p}$ as a product of connected groups, by the proof of [KWL, Proposition 1.2.3.11]), we have the Iwasawa decomposition $\mathrm{G}\left(\mathbb{Q}_{p}\right)=\mathrm{G}\left(\mathbb{Z}_{p}\right) \mathrm{P}\left(\mathbb{Q}_{p}\right)$, by [Bruhat and Tits 1972, Proposition 4.4.3] (see also [Casselman 1980, (18) on p. 392] for a more explicit statement). Consequently, $\mathscr{H}_{p}=\mathrm{G}\left(\mathbb{Z}_{p}\right)$ acts transitively on the set of possible filtrations $Z_{\mathbb{Z}_{p}}$ as above, and hence the assignment (A.4.5) is injective.

As for the surjectivity of (A.4.5), it suffices to show that, for some symplectic admissible filtration $\mathbb{Z}_{\mathbb{Z}_{p}}$, an isomorphism $\varphi_{-2, \mathbb{Z}_{p}}: \mathrm{Gr}_{-2} \stackrel{\mathbb{Z}_{\mathbb{Z}_{p}}}{\longrightarrow} \operatorname{Hom}_{\mathbb{Z}_{p}}\left(X \otimes_{\mathbb{Z}} \mathbb{Z}_{p}, \mathbb{Z}_{p}(1)\right)$ exists. By [Reiner 1975, Theorem 18.10] and [KWL, Corollary 1.1.2.6], it suffices to show that there exists some symplectic filtration $Z_{\mathbb{Q}_{p}}$ such that $Z_{\mathbb{Q}_{p},-2}$ and $\operatorname{Hom}_{\mathbb{Q}_{p}}\left(X \otimes_{\mathbb{Z}} \mathbb{Q}_{p}, \mathbb{Q}_{p}(1)\right)$ have the same 0 -multirank. Or, rather, we just need to notice that the $\mathbb{O}$-multirank of a totally isotropic $\mathbb{O} \otimes_{\mathbb{Z}} \mathbb{Q}_{p}$-submodule can be any 0 -multirank below a maximal one (with respect to the natural partial order), by Assumption A.2.1 and by the classification in [KWL, Proposition 1.2.3.7 and Corollary 1.2.3.10].

Lemma A.4.6. The assignment (A.4.1) is bijective.

Proof. It is already explained in the proof of Lemma A.4.4 that an isomorphism $\varphi_{-2, \mathbb{Z}_{p}}: \mathrm{Gr}_{-2}^{\mathrm{Z}_{\mathbb{Z}_{p}}} \stackrel{\sim}{\rightarrow} \operatorname{Hom}_{\mathbb{Z}_{p}}\left(X \otimes_{\mathbb{Z}} \mathbb{Z}_{p}, \mathbb{Z}_{p}(1)\right)$ exists for any $\mathbb{Z}_{\mathbb{Z}_{p}}$ considered there. Since $p$ is a good prime, which forces both $\left[L^{\#}: L\right]$ and $[X: \phi(Y)]$ to be prime to $p$, any choice of $\varphi_{-2, \mathbb{Z}_{p}}$ above uniquely determines an isomorphism $\varphi_{0}: \mathrm{Gr}_{0}^{\mathrm{Z}_{\mathbb{Z}_{p}}} \stackrel{\sim}{\longrightarrow} Y \otimes_{\mathbb{Z}} \mathbb{Z}_{p}$. Also, by the explicit classification in [KWL, Proposition 1.2.3.7 and Corollary 1.2.3.10] as in the proof of Lemma A.4.4, there exists a

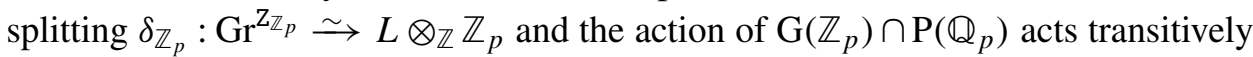
on the set of possible triples $\left(\varphi_{-2, \mathbb{Z}_{p}}, \varphi_{0, \mathbb{Z}_{p}}, \delta_{\mathbb{Z}_{p}}\right)$. Hence the assignment (A.4.1) is bijective, as desired. 
Lemma A.4.7. The assignment (A.4.2) is bijective.

Proof. By Lemma A.4.6, it suffices to show that (A.4.2) is injective. Suppose two representatives $\left(\mathrm{Z}_{\mathscr{H}}, \Phi_{\mathscr{H}}, \delta_{\mathscr{H}}\right)$ and $\left(\mathrm{Z}_{\mathscr{H}}^{\prime}, \Phi_{\mathscr{H}}^{\prime}, \delta_{\mathscr{H}}^{\prime}\right)$ with $\Phi_{\mathscr{H}}=\left(X, Y, \phi, \varphi_{-2, \mathscr{H}}, \varphi_{0, \mathscr{H}}\right)$ and $\Phi_{\mathscr{H}}^{\prime}=\left(X^{\prime}, Y^{\prime}, \phi^{\prime}, \varphi_{-2, \mathscr{H}}^{\prime}, \varphi_{0, \mathscr{H}}^{\prime}\right)$ are such that the induced ( $\left.\mathrm{Z}_{\mathscr{H} P}, \Phi_{\mathscr{H} P}, \delta_{\mathscr{H} P}\right)$ and $\left(\mathrm{Z}_{\mathscr{H} p}^{\prime}, \Phi_{\mathscr{H} p}^{\prime}, \delta_{\mathscr{H} p}^{\prime}\right)$ are equivalent to each other. By definition, $\mathrm{Z}_{\mathscr{H}^{p}}=\mathrm{Z}_{\mathscr{H} p}^{\prime}$, so that $\mathrm{Z}_{\mathscr{H}}=\mathrm{Z}_{\mathscr{H}}^{\prime}$ by Lemma A.4.4, and there exists a pair $\left(\gamma_{X}: X^{\prime} \stackrel{\sim}{\longrightarrow} X, \gamma_{Y}: Y \stackrel{\sim}{\longrightarrow} Y^{\prime}\right)$ matching $\Phi_{\mathscr{C}^{p}}$ with $\Phi_{\mathscr{H} p}^{\prime}$. Hence we may assume that $(X, Y, \phi)=\left(X^{\prime}, Y^{\prime}, \phi^{\prime}\right)$, take any $\mathrm{Z}$ in $\mathrm{Z}_{\mathscr{K}^{p}}=\mathrm{Z}_{\mathscr{H} p}^{\prime}$, and take any pairs

$$
\left(\varphi_{-2}: \mathrm{Gr}_{-2}^{\mathrm{Z}} \stackrel{\sim}{\longrightarrow} \operatorname{Hom}_{\hat{\mathbb{Z}}}\left(X \otimes_{\mathbb{Z}} \hat{\mathbb{Z}}, \hat{\mathbb{Z}}(1)\right), \varphi_{0}: \mathrm{Gr}_{0}^{\mathrm{Z}} \stackrel{\sim}{\longrightarrow} Y \otimes_{\mathbb{Z}} \hat{\mathbb{Z}}\right)
$$

and

$$
\left(\varphi_{-2}^{\prime}: \mathrm{Gr}_{-2}^{\mathrm{Z}} \stackrel{\sim}{\longrightarrow} \operatorname{Hom}_{\hat{\mathbb{Z}}}\left(X \otimes_{\mathbb{Z}} \hat{\mathbb{Z}}, \hat{\mathbb{Z}}(1)\right), \varphi_{0}^{\prime}: \mathrm{Gr}_{0}^{\mathrm{Z}} \stackrel{\sim}{\longrightarrow} Y \otimes_{\mathbb{Z}} \hat{\mathbb{Z}}\right)
$$

inducing $\left(\varphi_{-2, \mathscr{H}}, \varphi_{0, \mathscr{H}}\right)$ and $\left(\varphi_{-2, \mathscr{H}}^{\prime}, \varphi_{0, \mathscr{H}}^{\prime}\right)$, respectively, and inducing the same $\left(\varphi_{-2, \mathscr{H}^{p}}, \varphi_{0, \mathscr{H}^{p}}\right)$ and $\left(\varphi_{-2, \mathscr{H}^{p}}^{\prime}, \varphi_{0, \mathscr{H}^{p}}^{\prime}\right)$. Then the injectivity of (A.4.2) follows from that of (A.4.1).

Lemma A.4.8. If ( $\left.\mathrm{Z}_{\mathscr{H}^{p}}, \Phi_{\mathscr{H}}, \delta_{\mathscr{H} p}\right)$ is assigned to $\left(\mathrm{Z}_{\mathscr{H}}, \Phi_{\mathscr{H}}, \delta_{\mathscr{H}}\right)$ under (A.4.1), then we have a canonical isomorphism

$$
\Gamma_{\Phi_{\mathscr{H}}} \stackrel{\sim}{\longrightarrow} \Gamma_{\Phi_{\mathscr{H}} p}
$$

(see [KWL, Definition 6.2.4.1]). Moreover, we have a canonical isomorphism

$$
\boldsymbol{S}_{\Phi_{\mathscr{H}} p} \stackrel{\sim}{\longrightarrow} \boldsymbol{S}_{\Phi_{\mathscr{H}}},
$$

which induces a canonical isomorphism

$$
\left(S_{\Phi_{\mathscr{H}}}\right)_{\mathbb{R}}^{\vee} \stackrel{\sim}{\longrightarrow}\left(S_{\Phi_{\mathscr{F}} p}\right)_{\mathbb{R}}^{\vee}
$$

matching $\boldsymbol{P}_{\Phi_{\mathscr{H}}}$ with $\boldsymbol{P}_{\Phi_{\mathscr{F}} p}$ and $\boldsymbol{P}_{\Phi_{\mathscr{H}}}^{+}$with $\boldsymbol{P}_{\Phi_{\mathscr{H}} p}^{+}$, both isomorphisms being equivariant with the actions of the two sides of (A.4.9) above.

Proof. Since $p$ is a good prime, with $\mathscr{H}_{p}=\mathrm{G}\left(\mathbb{Z}_{p}\right)$, the levels at $p$ are not needed in the constructions of $\Gamma_{\Phi_{\mathscr{H}}}$ and $\boldsymbol{S}_{\Phi_{\mathscr{H}}}$ in [KWL, Sections 6.2.3-6.2.4], and hence we have the desired isomorphisms (A.4.9) and (A.4.10). The induced morphism (A.4.11) matches $\boldsymbol{P}_{\Phi_{\mathscr{H}}}$ with $\boldsymbol{P}_{\Phi_{\mathscr{H}} p}$ and $\boldsymbol{P}_{\Phi_{\mathscr{H}}}^{+}$with $\boldsymbol{P}_{\Phi_{\mathscr{H}} p}^{+}$because both sides of (A.4.11) can be canonically identified with the space of Hermitian forms over $Y \otimes_{\mathbb{Z}} \mathbb{R}$, as explained in the beginning of [KWL, Section 6.2.5], regardless of the levels $\mathscr{H}$ and $\mathscr{H}^{p}$.

Therefore, we also have assignments

$$
\left(\Phi_{\mathscr{H}}, \delta_{\mathscr{H}}, \sigma\right) \mapsto\left(\Phi_{\mathscr{H}^{p}}, \delta_{\mathscr{H}^{p}}, \sigma^{p}\right)
$$

and

$$
\left[\left(\Phi_{\mathscr{H}}, \delta_{\mathscr{H}}, \sigma\right)\right] \mapsto\left[\left(\Phi_{\mathscr{H}}, \delta_{\mathscr{H}^{p}}, \sigma^{p}\right)\right]
$$


(see [KWL, Definition 6.2.6.2]), which are compatible with (A.4.1) and (A.4.2). Here we have suppressed $Z_{\mathscr{H}}$ and $Z_{\mathscr{H}}$ from the notation; also, $\sigma \subset\left(S_{\Phi_{\mathscr{H}}}\right)_{\mathbb{R}}^{\vee}$ and $\sigma^{p} \subset\left(S_{\Phi_{\mathscr{x}} p}\right)_{\mathbb{R}}^{\vee}$ is the image of $\sigma$ under the isomorphism (A.4.11).

Lemma A.4.14. The assignment (A.4.12) is bijective.

Proof. This follows from Lemma A.4.6 and the definition of (A.4.12), based on Lemma A.4.8.

Lemma A.4.15. The assignment (A.4.13) is bijective.

Proof. By [KWL, Definition 6.2.6.2], given any representative $\left(\Phi_{\mathscr{H}}, \delta_{\mathscr{H}}\right)$ of a cusp label, the collection of the cones $\sigma \subset\left(S_{\Phi_{\mathscr{F}}}\right)_{\mathbb{R}}^{\vee}$ defining the same equivalence class $\left[\left(\Phi_{\mathscr{H}}, \delta_{\mathscr{H}}, \sigma\right)\right]$ form a $\Gamma_{\Phi_{\mathscr{H}}}$-orbit. Similarly, the collection of the cones $\sigma^{p} \subset\left(\boldsymbol{S}_{\Phi_{\mathscr{F}} p}\right)_{\mathbb{R}}^{\vee}$ defining the same equivalence class $\left[\left(\Phi_{\mathscr{H}^{p}}, \delta_{\mathscr{H}^{p}}, \sigma^{p}\right)\right]$ form a $\Gamma_{\Phi_{\mathscr{H}}}$-orbit. Hence, given (A.4.9), the lemma follows from Lemma A.4.7.

Definition A.4.16. $\Sigma$ is induced by $\Sigma^{p}$ if, for each cusp label $\left[\left(\mathrm{Z}_{\mathscr{H}}, \Phi_{\mathscr{H}}, \delta_{\mathscr{H}}\right)\right]$ of $\mathrm{M}_{\mathscr{H}}$ represented by some ( $\left.\mathrm{Z}_{\mathscr{H}}, \Phi_{\mathscr{H}}, \delta_{\mathscr{H}}\right)$, with assigned ( $\left.\mathrm{Z}_{\mathscr{H}}, \Phi_{\mathscr{H}^{p}}, \delta_{\mathscr{H}^{p}}\right)$ as in (A.4.1), the cone decomposition $\Sigma_{\Phi_{\mathscr{H}}}$ of $\boldsymbol{P}_{\Phi_{\mathscr{H}}}$ is the pullback of the cone decomposition $\Sigma_{\Phi_{\mathscr{H}} p}$ of $\boldsymbol{P}_{\Phi_{\mathscr{K} P}}$ under (A.4.11).

By forgetting the $p$-parts of level structures, we obtain a canonical isomorphism

$$
\mathrm{M}_{\mathscr{H}} \stackrel{\sim}{\rightarrow} \mathrm{M}_{\mathscr{H} p} \otimes_{\mathbb{Z}} \mathbb{Q}
$$

over $\mathrm{S}_{0, \mathbb{Q}}$ (as in [KWL, 1.4.4.1]), by [KWL, Proposition 1.4.4.3 and Remark 1.4.4.4] and by Assumption A.2.1. Given any $\Sigma^{p}$ for $\mathrm{M}_{\mathscr{H}^{p}}$, with induced $\Sigma$ for $\mathrm{M}_{\mathscr{H}}$ as in Definition A.4.16, by comparing the universal properties of $\mathrm{M}_{\mathscr{H}, \Sigma}^{\text {tor }}$ and $\mathrm{M}_{\mathscr{H}^{p}, \Sigma^{p}}^{\mathrm{tor}}$ as in [KWL, Theorem 6.4.1.1(5)-(6)], the isomorphism (A.4.17) above extends to a canonical isomorphism

$$
\mathrm{M}_{\mathscr{H}, \Sigma}^{\mathrm{tor}} \stackrel{\sim}{\longrightarrow} \mathrm{M}_{\mathscr{H}^{p}, \Sigma^{p}}^{\mathrm{tor}} \otimes_{\mathbb{Z}} \mathbb{Q}
$$

over $\mathrm{S}_{0, \mathbb{Q}}$, mapping $\mathrm{Z}_{\left[\left(\Phi_{\mathscr{H}}, \delta_{\mathscr{H}}, \sigma\right)\right]}$ isomorphically to $\mathrm{Z}_{\left[\left(\Phi_{\mathscr{F}}, \delta_{\mathscr{H}}, \sigma^{p}\right)\right]} \otimes_{\mathbb{Z}} \mathbb{Q}$ when $\left[\left(\Phi_{\mathscr{H}^{p}}, \delta_{\mathscr{H}^{p}}, \sigma^{p}\right)\right]$ is assigned to $\left[\left(\Phi_{\mathscr{H}}, \delta_{\mathscr{H}}, \sigma\right)\right]$ under (A.4.13), such that the pullback of the tautological semiabelian scheme over $\mathrm{M}_{\mathscr{H}^{p}, \Sigma^{p}}^{\mathrm{tor}} \otimes_{\mathbb{Z}} \mathbb{Q}$ is canonically isomorphic to the pullback of the tautological semiabelian scheme over $\mathrm{M}_{\mathscr{H}, \Sigma}^{\text {tor }}$. Consequently, by [KWL, Theorem 7.2.4.1(3)-(4)] and the fact that the pullback of the Hodge invertible sheaf over $\mathrm{M}_{\mathscr{H}^{p}, \Sigma^{p}}^{\text {tor }} \otimes_{\mathbb{Z}} \mathbb{Q}$ is canonically isomorphic to the pullback of the Hodge invertible sheaf over $\mathrm{M}_{\mathscr{H}, \Sigma}^{\text {tor }}$ (because their definitions only use the tautological semiabelian schemes), the canonical isomorphism (A.4.18) induces a canonical isomorphism

$$
\mathrm{M}_{\mathscr{H}}^{\min } \stackrel{\sim}{\longrightarrow} \mathrm{M}_{\mathscr{H} p}^{\min } \otimes_{\mathbb{Z}} \mathbb{Q}
$$

over $\mathrm{S}_{0, \mathbb{Q}}$, extending (A.4.17), compatible with (A.4.18) (under the canonical morphisms $\oint_{\mathscr{H}}: \mathrm{M}_{\mathscr{H}, \Sigma}^{\mathrm{tor}} \rightarrow \mathrm{M}_{\mathscr{H}}^{\min }$ and $\left.\oint_{\mathscr{H}^{p}} \otimes_{\mathbb{Z}} \mathbb{Q}: \mathrm{M}_{\mathscr{H}^{p}, \Sigma^{p}}^{\mathrm{tor}} \otimes_{\mathbb{Z}} \mathbb{Q} \rightarrow \mathrm{M}_{\mathscr{H}^{p}}^{\min } \otimes_{\mathbb{Z}} \mathbb{Q}\right)$, 
and mapping $Z_{\left[\left(\Phi_{\mathscr{K}}, \delta_{\mathscr{H}}\right)\right]}$ isomorphically to $\mathrm{Z}_{\left[\left(\Phi_{\mathscr{H}} p, \delta_{\mathscr{H} P}\right)\right]} \otimes_{\mathbb{Z}} \mathbb{Q}$ when $\left[\left(\Phi_{\mathscr{H}}, \delta_{\mathscr{H}^{p}}\right)\right]$ is assigned to $\left[\left(\Phi_{\mathscr{H}}, \delta_{\mathscr{H}}\right)\right]$ under (A.4.2) (where we have suppressed $\mathrm{Z}_{\mathscr{H}}$ and $\mathrm{Z}_{\mathscr{H} p}$ from the notation).

A.5. Complex analytic construction. By Proposition A.3.1, in order to prove Theorem A.2.2 we may and we shall assume that $\operatorname{char}(k(s))=0$. Thanks to the isomorphisms (A.4.17) and (A.4.19), we shall identify $U$ with a connected component of $\mathrm{M}_{\mathscr{H}} \otimes_{F_{0}} k(s), U^{\mathrm{min}}$ with the connected component of $\mathrm{M}_{\mathscr{H}}^{\min } \otimes_{F_{0}} k(s)$ that is the closure of $U$, and $U_{\left[\left(\Phi_{\mathscr{C} P}, \delta_{\mathscr{F} P}\right)\right]}$ with $U_{\left[\left(\Phi_{\mathscr{K}}, \delta_{\mathscr{F}}\right)\right]}$, the pullback of the stratum $\mathrm{Z}_{\left[\left(\Phi_{\mathscr{H}}, \delta \mathscr{H}\right)\right]}$ of $\mathrm{M}_{\mathscr{H}}^{\min }$ under the canonical morphism $U^{\mathrm{min}} \rightarrow \mathrm{M}_{\mathscr{H}}^{\min }$, when $\left[\left(\Phi_{\mathscr{H} p}, \delta_{\mathscr{H} p}\right)\right]$ is assigned to $\left[\left(\Phi_{\mathscr{H}}, \delta_{\mathscr{H}}\right)\right]$ under (A.4.2).

Now, in characteristic zero we no longer need $\mathscr{H}$ to be of the form $\mathscr{H}^{\prime} \mathscr{H}^{p} \mathscr{H}_{p}$ as in Section A.4. We shall allow $\mathscr{H}$ to be any neat open compact subgroup of $\mathrm{G}(\hat{\mathbb{Z}})$. Then $\mathrm{M}_{\mathscr{H}}$ and $\mathrm{M}_{\mathscr{H}}^{\min }$ are still defined over $\mathrm{M}_{0, \mathbb{Q}}=\operatorname{Spec}\left(F_{0}\right)$, with the stratification on the latter by locally closed subschemes $\mathrm{Z}_{\left[\left(\Phi_{\mathscr{H}}, \delta_{\mathscr{H}}\right)\right]}$ labeled by cusp labels $\left[\left(\Phi_{\mathscr{H}}, \delta_{\mathscr{H}}\right)\right]$ for $\mathrm{M}_{\mathscr{H C}}$ (see the same references as in Section A.2). For any geometric point $s \rightarrow \mathrm{S}_{0, \mathbb{Q}}$ with residue field $k(s)$ and for any connected component $U$ of the fiber $\mathrm{M}_{\mathscr{H}^{p}} \times \mathrm{S}_{0} s$, we define $U^{\mathrm{min}}$ to be the closure of $U$ in $\mathrm{M}_{\mathscr{H}}^{\min } \times \mathrm{s}_{0} s$ and $U_{\left[\left(\Phi_{\mathscr{H}}, \delta_{\mathscr{H}}\right)\right]}$ to be the pullback of $Z_{\left[\left(\Phi_{\mathscr{K}}, \delta_{\mathscr{H}}\right)\right]}$ to of $U^{\mathrm{min}}$ for each cusp label $\left[\left(\Phi_{\mathscr{H}}, \delta_{\mathscr{H}}\right)\right]$. (These are consistent with what we have done before, when the settings overlap.)

Then we have the following analogue of Theorem A.2.2:

Theorem A.5.1. With the setting as above, every stratum $U_{\left[\left(\Phi_{\mathscr{H}}, \delta \mathscr{H}\right)\right]}$ is nonempty.

Since $\mathrm{M}_{\mathscr{H}}^{\min }$ is projective over $\mathrm{S}_{0, \mathbb{Q}}$, we may and we shall assume that $k(s) \cong \mathbb{C}$. We shall denote base changes to $\mathbb{C}$ with a subscript, such as $\mathrm{M}_{\mathscr{H}, \mathbb{C}}=\mathrm{M}_{\mathscr{C}} \otimes_{F_{0}} \mathbb{C}$.

Let $X$ denote the $\mathrm{G}(\mathbb{R})$-orbit of $h_{0}$, which is a finite disjoint union of Hermitian symmetric domains, and let $\mathrm{X}_{0}$ denote the connected component of $\mathrm{X}$ containing $h_{0}$. Let $G(\mathbb{Q})_{0}$ denote the finite index subgroup of $G(\mathbb{Q})$ stabilizing $X_{0}$. Let Sh⿻ $\mathrm{G}(\mathbb{Q}) \backslash \mathrm{X} \times \mathrm{G}\left(\mathbb{A}^{\infty}\right) / \mathscr{H}$. By [Lan 2012, Lemma 2.5.1], we have a canonical bijection $\mathrm{G}(\mathbb{Q})_{0} \backslash \mathrm{X}_{0} \times \mathrm{G}\left(\mathbb{A}^{\infty}\right) / \mathscr{H} \rightarrow \mathrm{G}(\mathbb{Q}) \backslash \mathrm{X} \times \mathrm{G}\left(\mathbb{A}^{\infty}\right) / \mathscr{H}$. Let $\left\{g_{i}\right\}_{i \in I}$ be any finite set of elements of $\mathrm{G}\left(\mathbb{A}^{\infty}\right)$ such that $\mathrm{G}\left(\mathbb{A}^{\infty}\right)=\bigsqcup_{i \in I} \mathrm{G}(\mathbb{Q})_{0} h_{i} \mathcal{H}$, which exists because of [Borel 1963, Theorem 5.1] and because $G(\mathbb{Q})_{0}$ is of finite index in $G(\mathbb{Q})$. Then we have

$$
\mathrm{Sh}_{\mathscr{C}}=\mathrm{G}\left(\mathbb{Q}_{0}\right)_{0} \backslash \mathrm{X}_{0} \times \mathrm{G}\left(\mathbb{A}^{\infty}\right) / \mathscr{H}=\bigsqcup_{i \in I} \Gamma^{\left(g_{i}\right)} \backslash \mathrm{X}_{0},
$$

where $\Gamma^{\left(g_{i}\right)}:=\left(g_{i} \mathscr{H} g_{i}^{-1}\right) \cap \mathrm{G}(\mathbb{Q})_{0}$ for each $i \in I$. By applying [Baily and Borel 1966, Theorem 10.11] to each $\Gamma^{\left(g_{i}\right)} \backslash \mathrm{X}_{0}$, we obtain the minimal compactification $\mathrm{Sh}_{\mathscr{H}}^{\min }$ of $\mathrm{Sh}_{\mathscr{H}}$, which is the complex analytification of a normal projective variety $\mathrm{Sh}_{\mathscr{H}, \text { alg }}^{\min }$ over $\mathbb{C}$. Thus, Sh $\mathscr{H}$ is the analytification of a quasiprojective variety $\mathrm{Sh}_{\mathscr{H}, \text { alg }}$ (embedded in $\mathrm{Sh}_{\mathscr{H}, \text { alg }}^{\min }$ ). 
By [Lan 2012, Lemma 3.1.1], the rational boundary components $\mathrm{X}_{\mathrm{V}}$ of $\mathrm{X}_{0}$ (see [Baily and Borel 1966, Section 3.5]) correspond to parabolic subgroups of $G \otimes_{\mathbb{Z}} \mathbb{Q}$ stabilizing symplectic filtrations $\mathrm{V}$ on $L \otimes_{\mathbb{Z}} \mathbb{Q}$ with $\mathrm{V}_{-3}=0 \subset \mathrm{V}_{-2} \subset \mathrm{V}_{-1}=\mathrm{V}_{-2}^{\perp} \subset \mathrm{V}_{0}=$ $L \otimes_{\mathbb{Z}} \mathbb{Q}$. Consider the rational boundary components of $X \times G\left(\mathbb{A}^{\infty}\right)$ as in [Lan 2012, Definition 3.1.2], which are $\mathrm{G}(\mathbb{Q})$-orbits of pairs $(\mathrm{V}, g)$, where the $\mathrm{V}$ are as above and $g \in \mathrm{G}\left(\mathbb{A}^{\infty}\right)$. Consider the boundary components $\mathrm{G}(\mathbb{Q}) \backslash\left(\mathrm{G}(\mathbb{Q}) \mathrm{X}_{\mathrm{V}}\right) \times \mathrm{G}\left(\mathbb{A}^{\infty}\right) / \mathscr{H}=$ $\mathrm{G}(\mathbb{Q})_{0} \backslash\left(\mathrm{G}(\mathbb{Q})_{0} \mathrm{X}_{\mathrm{V}}\right) \times \mathrm{G}\left(\mathbb{A}^{\infty}\right) / \mathscr{H}$ of $S_{\mathscr{H}}=\mathrm{G}(\mathbb{Q})_{0} \backslash \mathrm{X}_{0} \times \mathrm{G}\left(\mathbb{A}^{\infty}\right) / \mathscr{H}$. By the construction in [Baily and Borel 1966], each such component defines a nonempty, locally closed subset and meets all connected components of $\mathrm{Sh}_{\mathscr{H}}^{\min }$, corresponding to a nonempty, locally closed subscheme of $\mathrm{Sh}_{\mathscr{H}, \text { alg }}^{\min }$, called its $\mathrm{G}(\mathbb{Q})(\mathrm{V}, g) \mathscr{H}$-stratum. Thus, we obtain the following:

Proposition A.5.3 (Satake, Baily-Borel). Each $\mathrm{G}(\mathbb{Q})(\mathrm{V}, g)$ He-stratum as above meets every connected component of $\mathrm{Sh}_{\mathscr{H}, \text { alg }}^{\min }$.

For each $g \in \mathrm{G}\left(\mathbb{A}^{\infty}\right)$, let $L^{(g)}$ denote the $\mathrm{O}$-lattice in $L \otimes_{\mathbb{Z}} \mathbb{Q}$ such that $L^{(g)} \otimes_{\mathbb{Z}} \hat{\mathbb{Z}}=$ $g\left(L \otimes_{\mathbb{Z}} \hat{\mathbb{Z}}\right)$ in $L \otimes_{\mathbb{Z}} \mathbb{A}^{\infty}$. Let $r \in \mathbb{Q}_{>0}^{\times}$be the unique element such that $v(g)=r u$ for some $u \in \hat{\mathbb{Z}}$, and let $\langle\cdot, \cdot\rangle^{(g)}: L^{(g)} \times L^{(g)} \rightarrow \mathbb{Z}(1)$ denote the pairing induced by $r\langle\cdot, \cdot\rangle \otimes_{\mathbb{Z}} \mathbb{Q}$ (see [Lan 2012, Section 2.4], the key point being that $\langle\cdot, \cdot\rangle^{(g)}$ is valued in $\mathbb{Z}(1))$.

Construction A.5.4. As explained in [Lan 2012, Section 3.1], we have an assignment of a fully symplectic admissible filtration $\mathrm{Z}^{(g)}$ on $\mathrm{Z} \otimes_{\mathbb{Z}} \hat{\mathbb{Z}}$ and a torus argument $\Phi^{(g)}=\left(X^{(g)}, Y^{(g)}, \phi^{(g)}, \varphi_{-2}^{(g)}, \varphi_{0}^{(g)}\right)$ to $\mathrm{G}(\mathbb{Q})(\mathrm{V}, g)$, by setting:

(1) $\mathrm{F}^{(g)}:=\left\{\mathrm{F}_{-i}^{(g)}:=\mathrm{V}_{-i} \cap L^{(g)}\right\}_{i \in \mathbb{Z}}$.

(2) $\mathrm{Z}^{(g)}:=\left\{\mathrm{Z}_{-i}^{(g)}:=g^{-1}\left(\mathrm{~F}_{-i}^{(g)} \otimes_{\mathbb{Z}} \hat{\mathbb{Z}}\right)\right\}_{i \in \mathbb{Z}}=\left\{g^{-1}\left(\mathrm{~V}_{-i} \otimes_{\mathbb{Q}} A^{\infty}\right) \cap\left(L \otimes_{\mathbb{Z}} \hat{\mathbb{Z}}\right)\right\}_{i \in \mathbb{Z}}$.

(3) $X^{(g)}:=\operatorname{Hom}_{\mathbb{Z}}\left(\mathrm{F}_{-2}^{(g)}, \mathbb{Z}(1)\right)=\operatorname{Hom}_{\mathbb{Z}}\left(\mathrm{Gr}_{-2}^{\mathrm{F}^{(g)}}, \mathbb{Z}(1)\right)$.

(4) $Y^{(g)}:=\mathrm{Gr}_{0}^{\mathrm{F}^{(g)}}=\mathrm{F}_{0}^{(g)} / \mathrm{F}_{-1}^{(g)}$.

(5) $\phi^{(g)}: Y^{(g)} \hookrightarrow X^{(g)}$, equivalent to the nondegenerate pairing

$$
\langle\cdot, \cdot\rangle_{20}^{(g)}: \mathrm{Gr}_{-2}^{\mathrm{F}^{(g)}} \times \mathrm{Gr}_{0}^{\mathrm{F}^{(g)}} \rightarrow \mathbb{Z}(1)
$$

induced by $\langle\cdot, \cdot\rangle^{(g)}: L^{(g)} \times L^{(g)} \rightarrow \mathbb{Z}(1)$.

(6) $\varphi_{-2}^{(g)}: \mathrm{Gr}_{-2}^{\mathrm{Z}^{(g)}} \stackrel{\sim}{\rightarrow} \operatorname{Hom}_{\hat{\mathbb{Z}}}\left(X^{(g)} \otimes_{\mathbb{Z}} \hat{\mathbb{Z}}, \hat{\mathbb{Z}}(1)\right)$, the composition

$$
\mathrm{Gr}_{-2}^{\mathrm{Z}^{(g)}} \stackrel{\mathrm{Gr}_{-2}(g)}{\longrightarrow} \mathrm{Gr}_{-2}^{\mathrm{F}^{(g)}} \otimes_{\mathbb{Z}} \hat{\mathbb{Z}} \stackrel{\sim}{\longrightarrow} \operatorname{Hom}_{\hat{\mathbb{Z}}}\left(X^{(g)} \otimes_{\mathbb{Z}} \hat{\mathbb{Z}}, \hat{\mathbb{Z}}(1)\right) .
$$

(7) $\varphi_{0}^{(g)}: \mathrm{Gr}_{0}^{\mathrm{Z}^{(g)}} \stackrel{\sim}{\longrightarrow} Y^{(g)} \otimes_{\mathbb{Z}} \hat{\mathbb{Z}}$, the composition

$$
\mathrm{Gr}_{0}^{\mathrm{r}^{(g)}} \stackrel{\stackrel{\mathrm{Gr}_{0}(g)}{\longrightarrow}}{\longrightarrow} \mathrm{Gr}_{0}^{\mathrm{F}^{(g)}} \otimes_{\mathbb{Z}} \hat{\mathbb{Z}} \stackrel{\sim}{\longrightarrow} Y^{(g)} \otimes_{\mathbb{Z}} \hat{\mathbb{Z}} .
$$


By the assumption that our integral PEL datum satisfies [Lan 2013a, Condition 1.4.3.10] and by the fact that maximal orders over Dedekind domains are hereditary (see [Reiner 1975, Theorem 21.4 and Corollary 21.5]), there exists a splitting $\varepsilon^{(g)}: \mathrm{Gr}^{\mathrm{F}^{(g)}} \stackrel{\sim}{\longrightarrow} L^{(g)}$, whose base extension from $\mathbb{Z}$ to $\hat{\mathbb{Z}}$ defines, by pre- and postcompositions with $\operatorname{Gr}(g)$ and $g^{-1}$, a splitting $\delta^{(g)}: \mathrm{Gr}^{\mathrm{(}^{(g)}} \stackrel{\sim}{\longrightarrow} L \otimes_{\mathbb{Z}} \hat{\mathbb{Z}}$. These define an assignment

$$
\mathrm{G}(\mathbb{Q})(\mathrm{V}, g) \mapsto\left[\left(\mathrm{Z}^{(g)}, \Phi^{(g)}, \delta^{(g)}\right)\right],
$$

which is compatible with the formation of $\mathscr{H}$-orbits and induces an assignment

$$
\mathrm{G}(\mathbb{Q})(\mathrm{V}, g) \mathscr{H} \mapsto\left[\left(\mathrm{Z}_{\mathscr{H}}^{(g)}, \Phi_{\mathscr{H}}^{(g)}, \delta_{\mathscr{H}}^{(g)}\right)\right] .
$$

Definition A.5.7. For each cusp label $\left[\left(\mathrm{Z}_{\mathscr{H}}, \Phi_{\mathscr{H}}, \delta_{\mathscr{H}}\right)\right]$, the $\left[\left(\Phi_{\mathscr{H}}, \delta_{\mathscr{H}}\right)\right]$-stratum of $\mathrm{Sh}_{\mathscr{H} \text {,alg }}^{\min }$ is the union of all the $\mathrm{G}(\mathbb{Q})(\mathrm{V}, g) \mathscr{H}$-strata such that $\left[\left(\mathrm{Z}_{\mathscr{H}}, \Phi_{\mathscr{H}}, \delta_{\mathscr{H}}\right)\right]$ is assigned to $\mathrm{G}(\mathbb{Q})(\mathrm{V}, g) \mathcal{H}$ under (A.5.6).

Proposition A.5.8. Given the $\mathscr{H}$-orbit $\mathrm{Z}_{\mathscr{H}}$ of any $\mathrm{Z}=\left\{\mathrm{Z}_{-i}\right\}_{i \in \mathbb{Z}}$ as above, there exists some totally isotropic $\mathbb{O} \otimes_{\mathbb{Z}} \mathbb{Q}$-submodule $\mathrm{V}_{-2}$ of $L \otimes_{\mathbb{Z}} \mathbb{Q}$ such that $\mathrm{V}_{-2} \otimes_{\mathbb{Q}} \mathbb{A}^{\infty}$ lies in the $\mathscr{H}$-orbit of $\mathrm{Z}_{-2} \otimes_{\mathbb{Z}} \mathbb{Q}$.

Proof. Up to replacing $\mathscr{H}$ with an open compact subgroup, which is harmless for proving this proposition, we may and we shall assume that $\mathscr{H}=\mathscr{H}^{S} \mathscr{H}_{S}$, where $S$ is a finite set of primes containing all bad ones for the integral PEL datum (see [KWL, Definition 1.4.1.1]), such that $\mathscr{H}^{S}=\mathrm{G}\left(\hat{\mathbb{Z}}^{S}\right)=\prod_{\ell \notin S} \mathrm{G}\left(\mathbb{Z}_{\ell}\right)$ and $\mathscr{H}_{S} \subset \mathrm{G}\left(\hat{\mathbb{Z}}_{S}\right)=$ $\prod_{\ell \in S} \mathrm{G}\left(\mathbb{Z}_{\ell}\right)$, where $\ell \notin S$ means that $\ell$ runs through all prime numbers not in $S$.

By Assumption A.2.1, by reduction to the case where $0 \otimes_{\mathbb{Z}} \mathbb{Q}$ is a product of division algebras, by Morita equivalence (see [KWL, Proposition 1.2.1.14]) and, by the local-global principle for isotropy in [Scharlau 1985, table on p. 347 and its references], it follows that, if $Z_{-2} \otimes_{\mathbb{Z}} \mathbb{Q}$ is nonzero and extends to some isotropic $0 \otimes_{\mathbb{Z}} \mathbb{A}$-submodule of $L \otimes_{\mathbb{Z}} \mathbb{A}$ isomorphic to the base extension of some 0 -lattice, then there exists some nonzero isotropic element in $L \otimes_{\mathbb{Z}} \mathbb{Q}$. By induction on the 0 -multirank of $Z_{-2} \otimes_{\mathbb{Z}} \mathbb{Q}$ - by replacing $L \otimes_{\mathbb{Z}} \mathbb{Q}$ (resp. $L \otimes_{\mathbb{Z}} \mathbb{A}^{\infty}$ ) with the orthogonal complement modulo the span of a nonzero isotropic element in $L \otimes_{\mathbb{Z}} \mathbb{Q}$ (resp. $L \otimes_{\mathbb{Z}} \mathbb{A}^{\infty}$ ) - there exists some totally isotropic $0 \otimes_{\mathbb{Z}} \mathbb{Q}$-submodule $\mathrm{V}_{-2}^{0}$ of $L \otimes_{\mathbb{Z}} \mathbb{Q}$ such that $\mathrm{V}_{-2}^{0} \otimes_{\mathbb{Q}} \mathbb{A}^{\infty}$ and $Z_{-2} \otimes_{\mathbb{Z}} \mathbb{Q}$ have the same $\mathbb{0}$-multirank.

Let $\mathrm{G}^{\prime}$ denote the derived subgroup of $\mathrm{G} \otimes_{\mathbb{Z}} \mathbb{Q}$ (see [SGA 3 I 1970, Définition 7.2(vii), p. 364 and Corollaire 7.10, p. 373]). Then the pullback to $G^{\prime}$ induces a bijection between the parabolic subgroups of $\mathrm{G} \otimes_{\mathbb{Z}} \mathbb{Q}$ and those of $\mathrm{G}^{\prime}$ (see [SGA 3 III 1970, Propositions 6.2.4 and 6.2.8, pp. 264-266; Springer 1998, Theorem 15.1.2(ii) and Theorem 15.4.6(i)]), and they both are in bijection with the stabilizers of flags of totally isotropic $\mathbb{O} \otimes_{\mathbb{Z}} \mathbb{Q}$-submodules, as in Lemma A.4.3. Therefore, there exists some element $h=\left(h_{\ell}\right) \in \mathrm{G}^{\prime}\left(\mathbb{A}^{\infty}\right)$, where the index $\ell$ runs through all prime numbers, such that $\mathrm{V}_{-2}^{0} \otimes_{\mathbb{Q}} \mathbb{A}^{\infty}=h\left(\mathrm{Z}_{-2} \otimes_{\mathbb{Z}} \mathbb{Q}\right)$. 
Since $\mathrm{G}^{\prime}$ is simply connected, by Assumption A.2.1 (because the kernel of the similitude character of $G \otimes_{\mathbb{Z}} \mathbb{Q}$ factorizes over an algebraic closure of $\mathbb{Q}$ as a product of groups with simply connected derived groups, by the proof of [KWL, Proposition 1.2.3.11]), by weak approximation (see [Platonov and Rapinchuk 1994, Theorem 7.8]) there exists $\gamma \in \mathrm{G}^{\prime}(\mathbb{Q})$ such that $\gamma\left(h_{\ell}\right)_{\ell \in S} \in \mathscr{H}_{S}$. On the other hand, by using the Iwasawa decomposition at the places $\ell \in S$ as in the proof of Lemma A.4.4, up to replacing $h_{\ell}$ with a right-multiple of $h_{\ell}$ by an element of $\mathrm{G}^{\prime}\left(\mathbb{Q}_{\ell}\right)$ stabilizing $\mathrm{Z}_{-2} \otimes_{\hat{\mathbb{Z}}} \mathbb{Q}_{\ell}$, we may assume that $\gamma h_{\ell} \in \mathrm{G}\left(\mathbb{Z}_{\ell}\right)$ for all $\ell \notin S$. Thus, we can conclude by taking $\mathrm{V}_{-2}:=\gamma\left(\mathrm{V}_{-2}^{0}\right)$.

Proposition A.5.9. For each cusp label $\left[\left(\mathrm{Z}_{\mathscr{H}}, \Phi_{\mathscr{H}}, \delta_{\mathscr{H}}\right)\right]$, there exists some rational boundary component $\mathrm{G}(\mathbb{Q})(\mathrm{V}, g)$ of $\mathrm{X} \times \mathrm{G}\left(\mathbb{A}^{\infty}\right)$ such that $\left[\left(\mathrm{Z}_{\mathscr{H}}, \Phi_{\mathscr{H}}, \delta_{\mathscr{H}}\right)\right]$ is assigned to $\mathrm{G}(\mathbb{Q})(\mathrm{V}, g) \mathscr{H}$ under (A.5.6).

Proof. Let $\left(\mathrm{Z}, \Phi=\left(X, Y, \phi, \varphi_{-2}, \varphi_{0}\right), \delta\right)$ be any triple whose $\mathcal{H}$-orbit induces $\left[\left(\mathrm{Z}_{\mathscr{H}}, \Phi_{\mathscr{H}}, \delta_{\mathscr{H}}\right)\right]$ and let $\mathrm{V}_{-2}$ be as in Proposition A.5.8. Up to replacing $(\mathrm{Z}, \Phi, \delta)$ with another such triple, we may and we shall assume that

$$
\mathrm{Z}_{-2}=\left(\mathrm{V}_{-2} \otimes_{\mathbb{Q}} \mathbb{A}^{\infty}\right) \cap\left(L \otimes_{\mathbb{Z}} \hat{\mathbb{Z}}\right)=\mathrm{Z}_{-2}^{(1)},
$$

where $\mathrm{F}^{(1)}=\left\{\mathrm{F}_{-i}^{(1)}\right\}_{i \in \mathbb{Z}}, \mathrm{Z}^{(1)}=\left\{\mathrm{Z}_{-i}^{(1)}\right\}_{i \in \mathbb{Z}}$ and $\Phi^{(1)}=\left(X^{(1)}, Y^{(1)}, \phi^{(1)}, \varphi_{-2}^{(1)}, \varphi_{0}^{(1)}\right)$ are assigned to $(\mathrm{V}, 1)$ as in Construction A.5.4, together with some noncanonical choices of $\varepsilon^{(1)}$ and $\delta^{(1)}$.

Let $\mathrm{P}$ denote the parabolic subgroup of $\mathrm{G} \otimes_{\mathbb{Z}} \mathbb{Q}$ stabilizing $\mathrm{V}_{-2}$ (see Lemma A.4.3). By (A.5.10), the elements of $\mathrm{P}\left(\mathbb{A}^{\infty}\right)$ also stabilize $\mathrm{Z}_{-2} \otimes_{\mathbb{Z}} \mathbb{Q}$. Therefore, for each $g \in \mathrm{P}\left(\mathbb{A}^{\infty}\right)$, the filtration $\mathrm{Z}^{(g)}$ defined as in Construction A.5.4 coincides with Z.

Using (A.5.10) and the compatibility among the objects, both $\phi \otimes_{\mathbb{Z}} \hat{\mathbb{Z}}$ and $\phi^{(1)} \otimes_{\mathbb{Z}} \hat{\mathbb{Z}}$ can be identified (under $\left(\varphi_{-2}, \varphi_{0}\right)$ and $\left.\left(\varphi_{-2}^{(1)}, \varphi_{0}^{(1)}\right)\right)$ with the canonical morphism

$$
\langle\cdot, \cdot\rangle_{20}^{*}: \mathrm{Gr}_{0}^{\mathrm{Z}} \rightarrow \operatorname{Hom}_{\hat{\mathbb{Z}}}\left(\mathrm{Gr}_{-2}^{\mathrm{Z}}, \hat{\mathbb{Z}}(1)\right)
$$

induced by the pairing $\langle\cdot, \cdot\rangle$, which induce compatible isomorphisms

$$
{ }^{t}\left(\varphi_{-2}^{(1)} \circ \varphi_{-2}^{-1}\right): X^{(1)} \otimes_{\mathbb{Z}} \hat{\mathbb{Z}} \stackrel{\sim}{\longrightarrow} X \otimes_{\mathbb{Z}} \hat{\mathbb{Z}}
$$

and

$$
\varphi_{0}^{(1)} \circ \varphi_{0}^{-1}: Y \otimes_{\mathbb{Z}} \hat{\mathbb{Z}} \stackrel{\sim}{\longrightarrow} Y^{(1)} \otimes_{\mathbb{Z}} \hat{\mathbb{Z}} .
$$

By [KWL, Condition 1.4.3.10], there exists some maximal order $\mathbb{O}^{\prime}$ in $O \otimes_{\mathbb{Z}} \mathbb{Q}$, containing $\mathrm{O}$, such that the $\mathrm{O}$-action on $L$ extends to an $\mathrm{O}^{\prime}$-action; hence the $\mathrm{O}$-actions on $Y$ and $Y^{(1)}$ also extend to $\mathrm{O}^{\prime}$-actions. Using the local isomorphisms given by (A.5.13), by [Reiner 1975, Theorem 18.10] (which is applicable because we are now considering modules of the maximal order $\mathrm{O}^{\prime}$ ) and $[\mathrm{KWL}$, Corollary 1.1.2.6] there exists an element $g_{0} \in \mathrm{GL}_{\mathbb{O}_{\mathbb{Z}} \mathbb{A}}\left(\mathrm{Gr}_{0}^{Z} \otimes_{\mathbb{Z}} \mathbb{Q}\right)$ and an $\mathbb{O}$-equivariant 
embedding $h_{0}: Y^{(1)} \hookrightarrow Y \otimes_{\mathbb{Z}} \mathbb{Q}$ such that $\left(h_{0}\left(Y^{(1)}\right)\right) \otimes_{\mathbb{Z}} \hat{\mathbb{Z}}=\left(\varphi_{0} \otimes_{\mathbb{Z}} \mathbb{Q}\right)\left(g_{0}\left(\mathrm{Gr}_{0}^{Z}\right)\right)$

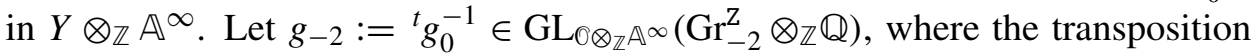
is induced by (A.5.11). Then there is a corresponding 0 -equivariant embedding $h_{-2}: \operatorname{Hom}_{\mathbb{Z}}\left(X^{(1)}, \mathbb{Z}(1)\right) \hookrightarrow \operatorname{Hom}_{\mathbb{Z}}(X, \mathbb{Z}(1)) \otimes_{\mathbb{Z}} \mathbb{Q}$ such that

$$
\left(h_{-2}\left(\operatorname{Hom}_{\mathbb{Z}}\left(X^{(1)}, \mathbb{Z}(1)\right)\right)\right) \otimes_{\mathbb{Z}} \hat{\mathbb{Z}}=\left(\varphi_{-2} \otimes_{\mathbb{Z}} \mathbb{Q}\right)\left(g_{-2}\left(\mathrm{Gr}_{-2}^{\mathrm{Z}}\right)\right)
$$

in $\operatorname{Hom}_{\mathbb{Z}}(X, \mathbb{Z}(1)) \otimes_{\mathbb{Z}} \mathbb{A}^{\infty}$.

Take $g \in \mathrm{P}\left(\mathbb{A}^{\infty}\right)$ such that $\mathrm{Gr}_{-2}(g)=g_{-2}, \mathrm{Gr}_{0}(g)=g_{0}$, and $v(g)=1$, which exists thanks to the splitting $\delta$. Then $X^{(g)}$ and $Y^{(g)}$ are realized as the preimages of $X$ and $Y$ under ${ }^{t} h_{-2} \otimes_{\mathbb{Z}} \mathbb{Q}$ and $h_{0}^{-1} \otimes_{\mathbb{Z}} \mathbb{Q}$, respectively, and the induced pair $\left(\gamma_{X}: X^{(g)} \stackrel{\sim}{\longrightarrow} X, \gamma_{Y}: Y \stackrel{\sim}{\longrightarrow} Y^{(g)}\right)$ matches $\Phi^{(g)}$ with $\Phi$. Such a $(\mathrm{V}, g)$ is what we want.

As explained in [Lan 2012, Section 2.5], there is a canonical open and closed immersion

$$
\mathrm{Sh}_{\mathscr{H}, \text { alg }} \hookrightarrow \mathrm{M}_{\mathcal{H}, \mathbb{C}} .
$$

As explained in [Kottwitz 1992, §8, p. 399] (see also [KWL, Remark 1.4.3.12]), $\mathrm{M}_{\mathcal{H}, \mathbb{C}}$ is the disjoint union of the images of morphisms like (A.5.14), from certain $\mathrm{Sh}_{\mathscr{H}, \text { alg }}^{(j)}$ defined by some $\left(\mathcal{O}, \star, L^{(j)},\langle\cdot, \cdot\rangle^{(j)}, h_{0}\right)$ such that $\left(L^{(j)},\langle\cdot, \cdot\rangle^{(j)}\right) \otimes_{\mathbb{Z}} \hat{\mathbb{Z}} \cong$ $(L,\langle\cdot, \cdot\rangle) \otimes_{\mathbb{Z}} \hat{\mathbb{Z}}$ and $\left(L^{(j)},\langle\cdot, \cdot\rangle^{(j)}\right) \otimes_{\mathbb{Z}} \mathbb{R} \cong(L,\langle\cdot, \cdot\rangle) \otimes_{\mathbb{Z}} \mathbb{R}$, but not necessarily satisfying $\left(L^{(j)},\langle\cdot, \cdot\rangle^{(j)}\right) \otimes_{\mathbb{Z}} \mathbb{Q} \cong(L,\langle\cdot, \cdot\rangle) \otimes_{\mathbb{Z}} \mathbb{Q}$, for all $j$ in some index set $J$ (whose precise description is not important for our purpose). (Each $\left(L^{(j)},\langle\cdot, \cdot\rangle^{(j)}\right)$ is determined by its rational version $\left(L^{(j)},\langle\cdot, \cdot\rangle^{(j)}\right) \otimes_{\mathbb{Z}} \mathbb{Q}$ by taking the intersection of the latter with $\left(L^{(j)},\langle\cdot, \cdot\rangle^{(j)}\right) \otimes_{\mathbb{Z}} \hat{\mathbb{Z}} \cong(L,\langle\cdot, \cdot\rangle) \otimes_{\mathbb{Z}} \hat{\mathbb{Z}}$ in $\left(L^{(j)},\langle\cdot, \cdot\rangle^{(j)}\right) \otimes_{\mathbb{Z}} \mathbb{A}^{\infty} \cong$ $(L,\langle\cdot, \cdot\rangle) \otimes_{\mathbb{Z}} \mathbb{A}^{\infty}$. Due to the failure of Hasse's principle, $J$ might have more than one element.)

By [Lan 2012, Theorem 5.1.1], (A.5.14) extends to a canonical open and closed immersion

$$
\mathrm{Sh}_{\mathscr{H}, \text { alg }}^{\min } \hookrightarrow \mathrm{M}_{\mathscr{H}, \mathbb{C}}^{\min }
$$

respecting the stratifications on both sides labeled by cusp labels (see Definition A.5.7). Again, $M_{\mathscr{H}, \mathbb{C}}^{\min }$ is the disjoint union of the images of morphisms like (A.5.15), from the minimal compactifications $\operatorname{Sh}_{\mathscr{H}, \text { alg }}^{(j), \text { min }}$ of $\operatorname{Sh}_{\mathscr{H}, \text { alg }}^{(j)}$ for all $j \in J$.

Everything we have proved remains true after replacing the objects defined by $(L,\langle\cdot, \cdot\rangle)$ with those defined by $\left(L^{(j)},\langle\cdot, \cdot\rangle^{(j)}\right)$ for each $j \in J$. Thus, in order to show that $U_{\left[\left(\Phi_{\mathscr{H}}, \delta \mathscr{H}\right)\right]}$ is nonempty, it suffices to note that, by Propositions A.5.3 and A.5.9, the $\left[\left(\Phi_{\mathscr{H}}, \delta_{\mathscr{H}}\right)\right]$-stratum of $S_{\mathscr{H}, \text { alg }}^{(j), \text { min }}$ meets every connected component of $\mathrm{Sh}_{\mathscr{H}, \text { alg }}^{(j), \min }$ for all $j \in J$. The proof of Theorem A.5.1 is now complete.

By Proposition A.3.1, and by the explanations in Section A.4 and in the beginning of this section, the proof of Theorem A.2.2 is also complete. 
A.6. Extension to cases of ramified characteristics. In this section, we shall no longer assume that $p$ is a good prime for the integral PEL datum $\left(\mathcal{O}, \star, L,\langle\cdot, \cdot\rangle, h_{0}\right)$, but we shall assume that the image $\mathscr{H}^{p}$ of $\mathscr{H}^{\mathcal{H}}$ under the canonical homomorphism $\mathrm{G}(\hat{\mathbb{Z}}) \rightarrow \mathrm{G}\left(\hat{\mathbb{Z}}^{p}\right)$ is neat.

Even for such general $\mathscr{H}$ and $p$, for any collections of lattices stabilized by $\mathscr{H}$ as in [Lan 2014, Section 2] we still have an integral model $\overrightarrow{\mathrm{M}}_{\mathscr{H}}$ of $\mathrm{M}_{\mathscr{H}}$ that is flat over $\mathrm{S}_{0}$, constructed by "taking normalization" (see [Lan 2014, Proposition 6.1 and also the introduction]). Moreover, we have an integral model $\overrightarrow{\mathrm{M}}_{\mathscr{H}}^{\min }$ of $\mathrm{M}_{\mathscr{H}}^{\min }$ that is projective and flat over $S_{0}$ (see [Lan 2014, Proposition 6.4]), with a stratification by locally closed subschemes $\vec{Z}_{\left[\left(\Phi_{\mathscr{H}}, \delta \mathscr{H}\right)\right]}$ labeled by cusp labels $\left[\left(\Phi_{\mathscr{H}}, \delta_{\mathscr{H}}\right)\right]$ for $\mathrm{M}_{\mathscr{H}}$, which extends the stratification of $\mathrm{M}_{\mathscr{H}}$ by the locally closed subschemes $\mathrm{Z}_{\left[\left(\Phi_{\mathscr{H}}, \delta \mathscr{H}\right)\right]}$ (see [Lan 2014, Theorem 12.1]). For certain (possibly nonsmooth) compatible collections $\Sigma$ (not the same ones for which we can construct $M_{\mathscr{H}, \Sigma}^{\text {tor }}$ over $M_{0, \mathbb{Q}}$ ), we also have the toroidal compactifications $\overrightarrow{\mathrm{M}}_{\mathscr{H}, \Sigma}^{\text {tor }}$ of $\overrightarrow{\mathrm{M}}_{\mathscr{H}}$ that are projective and flat over $S_{0}$ (see [Lan 2014, Section 7]), with a stratification by locally closed subschemes $\vec{Z}_{\left[\left(\Phi_{\mathscr{H}}, \delta \mathscr{H}, \sigma\right)\right]}$ (see [Lan 2014, Theorem 9.13]) and a canonical surjection $\vec{\oint}_{\mathscr{H}}: \overrightarrow{\mathrm{M}}_{\mathscr{H}, \Sigma}^{\mathrm{tor}} \rightarrow \overrightarrow{\mathrm{M}}_{\mathscr{H}}^{\text {min }}$ with geometrically connected fibers (see [Lan 2014, Lemma 12.9 and its proof $]$ ), inducing surjections $\vec{Z}_{\left[\left(\Phi_{\mathscr{H}}, \delta \mathscr{H}, \sigma\right)\right]} \rightarrow \vec{Z}_{\left[\left(\Phi_{\mathscr{H}}, \delta_{\mathscr{H}}\right)\right]}$ (see [Lan 2014, Theorem 12.16]).

As in Section A.2, consider a geometric point $s \rightarrow \mathrm{S}_{0}=\operatorname{Spec}\left(\mathcal{O}_{F_{0},(p)}\right)$ with algebraically closed residue field $k(s)$ and consider a connected component $U^{\mathrm{min}}$ of the fiber $\overrightarrow{\mathrm{M}}_{\mathscr{H}}^{\min } \times_{\mathrm{S}_{0}} s$. For each cusp label $\left[\left(\Phi_{\mathscr{H}}, \delta_{\mathscr{H}}\right)\right]$ for $\mathrm{M}_{\mathscr{H}}$, we define $U_{\left[\left(\Phi_{\mathscr{H}}, \delta_{\mathscr{H}}\right)\right]}$ to be the pullback of $\vec{Z}_{\left[\left(\Phi_{\mathscr{H}}, \delta_{\mathscr{H}}\right)\right]}$ to $U^{\mathrm{min}}$. Since the fibers of $\vec{\oint}_{\mathscr{H}}$ are geometrically connected, the preimage of $U^{\text {min }}$ under $\vec{\oint}_{\mathscr{H}} \times \mathrm{s}_{0} s$ is a connected component $U^{\text {tor }}$ of $\overrightarrow{\mathrm{M}}_{\mathscr{H}, \Sigma}^{\text {tor }} \times \mathrm{S}_{0} s$. (In general, neither $\overrightarrow{\mathrm{M}}_{\mathscr{H}}^{\min } \times{ }_{\mathrm{S}_{0}} s$ nor $\overrightarrow{\mathrm{M}}_{\mathscr{H}, \Sigma}^{\text {tor }} \times \mathrm{S}_{0} s$ is normal.) For each equivalence class $\left[\left(\Phi_{\mathscr{H}}, \delta_{\mathscr{H}}, \sigma\right)\right]$ defining a stratum $\mathrm{Z}_{\left[\left(\Phi_{\mathscr{H}}, \delta_{\mathscr{H}}, \sigma\right)\right]}$ of $\overrightarrow{\mathrm{M}}_{\mathscr{H}, \Sigma}^{\text {tor }}$, we define $U_{\left[\left(\Phi_{\mathscr{H}}, \delta_{\mathscr{H}}, \sigma\right)\right]}$ to be the pullback of $\overrightarrow{\mathrm{Z}}_{\left[\left(\Phi_{\mathscr{H}}, \delta_{\mathscr{H}}, \sigma\right)\right]}$. Then we also have a canonical surjection $U_{\left[\left(\Phi_{\mathscr{H}}, \delta_{\mathscr{H}}, \sigma\right)\right]} \rightarrow U_{\left[\left(\Phi_{\mathscr{H}}, \delta_{\mathscr{H}}\right)\right]}$ induced by $\oint_{\mathscr{H}}$.

Theorem A.6.1. With the setting as above, all strata of $U^{\mathrm{min}}$ are nonempty.

By using the canonical surjection $U_{\left[\left(\Phi_{\mathscr{Y}}, \delta_{\mathscr{Y}}, \sigma\right)\right]} \rightarrow U_{\left[\left(\Phi_{\mathscr{Y}}, \delta_{\mathscr{Y}}\right)\right]}$ (as in the proof of Corollary A.2.3), Theorem A.6.1 implies the following:

Corollary A.6.2. With the setting as above, all strata of $U^{\text {tor }}$ are nonempty.

As in Section A.3, it suffices to prove the following:

Proposition A.6.3. Suppose Theorem A.6.1 is true when $\operatorname{char}(k(s))=0$. Then it is also true when $\operatorname{char}(k(s))=p>0$.

Remark A.6.4. Since $\overrightarrow{M_{\mathscr{H}}} \otimes_{\mathbb{Z}} \mathbb{Q} \cong M_{\mathscr{H}}$ and $\vec{M}_{\mathscr{H}}^{\min } \otimes_{\mathbb{Z}} \mathbb{Q} \cong M_{\mathscr{H}}^{\min }$ by construction, by Theorem A.5.1 the assumption in Proposition A.6.3 always holds. Nevertheless, the 
proof of Proposition A.6.3 will clarify that the deduction of Theorem A.6.1 from Theorem A.5.1 does not require Assumption A.2.1 (cf. Remark A.3.2).

The remainder of this section will be devoted to the proof of Proposition A.6.3. We shall assume that $\operatorname{char}(k(s))=p>0$.

While each $Z_{\left[\left(\Phi_{\mathscr{F}}, \delta_{\mathscr{F}}\right)\right]}$ is isomorphic to some boundary moduli problem $\mathrm{M}_{\mathscr{H}}^{Z_{\mathscr{H}}}$, each stratum $\vec{Z}_{\left[\left(\Phi_{\mathscr{K}}, \delta_{\mathscr{H}}\right)\right]}$ of $\overrightarrow{\mathrm{M}}_{\mathscr{H}}^{\mathrm{min}}$ is similarly isomorphic to some integral model $\overrightarrow{\mathrm{M}}_{\mathscr{H}}^{Z_{\mathscr{K}}}$ defined by taking normalization (see [Lan 2014, Proposition 7.4 and Theorems 12.1 and 12.16]). Hence it also makes sense to consider the minimal compactification $\vec{Z}_{\left[\left(\Phi_{\mathscr{Y}}, \delta_{\mathscr{Y}}\right)\right]}$ of $\vec{Z}_{\left[\left(\Phi_{\mathscr{K}}, \delta_{\mathscr{Y}}\right)\right]}$, which is proper flat (with possibly nonnormal geometric fibers) over $\mathrm{S}_{0}$, and we obtain the following:

Lemma A.6.5 (cf. Lemma A.3.4 and [Deligne and Mumford 1969, Theorem 4.17(ii)]). There exists some discrete valuation ring $R$ that is flat over $O_{F_{0}},(p)$, with fraction field $K$ and residue field $k(s)$, the latter lifting the structural homomorphism $\mathcal{O}_{F_{0},(p)} \rightarrow k(s)$, such that, for each cusp label $\left[\left(\Phi_{\mathscr{H}}, \delta_{\mathscr{H}}\right)\right]$ and each connected component $V$ of $\vec{Z}_{\left[\left(\Phi_{\mathscr{H}}, \delta_{\mathscr{H}}\right)\right]} \otimes_{\mathcal{O}_{F_{0},(p)}} R$, the induced flat morphism $V \rightarrow \operatorname{Spec}(R)$ has connected special fiber over $\operatorname{Spec}(k(s))$.

Proof of Proposition A.6.3. By [Lan 2014, Corollary 12.4], it suffices to show that $U_{\left[\left(\Phi_{\mathscr{H}}, \delta_{\mathscr{H}}\right)\right]} \neq \varnothing$ when $\left[\left(\Phi_{\mathscr{H}}, \delta_{\mathscr{H}}\right)\right]$ is maximal with respect to the surjection relations, as in [KWL, Definition 5.4.2.13]. In this case, by [Lan 2014, Theorem 12.1], $\vec{Z}_{\left[\left(\Phi_{\mathscr{K}}, \delta \mathscr{F}\right)\right]}$ is a closed stratum of $\overrightarrow{\mathrm{M}}_{\mathscr{H}}^{\min }$ and so $\vec{Z}_{\left[\left(\Phi_{\mathscr{H}}, \delta_{\mathscr{H}}\right)\right]}=\vec{Z}_{\left[\left(\Phi_{\mathscr{H}}, \delta_{\mathscr{H}}\right)\right]}$. Hence the lemma follows from Theorem A.5.1 and the same argument as in the proof of Proposition A.3.1, with the reference to Lemma A.3.4 replaced with an analogous reference to Lemma A.6.5.

As explained in Remark A.6.4, the proof of Theorem A.6.1 is now complete.

\section{A.7. Examples.}

Example A.7.1. Suppose $O \otimes_{\mathbb{Z}} \mathbb{Q}$ is a $C M$ field $F$ with maximal totally subfield $F^{+}$, with positive involution given by the complex conjugation of $F$ over $F^{+}$. Suppose $L=\mathcal{O}_{F}^{\oplus a+b}$, where $a \geq b \geq 0$ are integers. Suppose $(2 \pi \sqrt{-1})^{-1}\langle\cdot, \cdot\rangle$ is the skew-Hermitian pairing defined in block matrix form

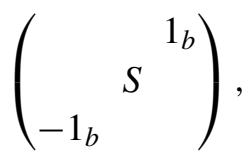

where $S$ is some $(a-b) \times(a-b)$ matrix over $F$ such that $\sqrt{-1} S$ is Hermitian and either positive or negative definite. Then, for each $0 \leq r \leq b$, the $\mathrm{O}$-submodule $\mathrm{Z}_{-2}^{(r)}$ of $L=\mathrm{O}_{F}^{\oplus(a+b)}$ with the last $a+b-r$ entries zero is totally isotropic, and $\mathrm{V}_{-2}^{(r)}:=$ $\mathrm{F}_{-2}^{(r)} \otimes_{\mathbb{Z}} \mathbb{Q}$ is a totally isotropic $F$-submodule of $L \otimes_{\mathbb{Z}} \mathbb{Q}=F^{\oplus(a+b)}$, which is maximal when $r=b$. The stabilizer of $\mathrm{V}_{-2}^{(r)}$ either is the whole group (when $r=0$ ) or defines a 
maximal (proper) parabolic subgroup $\mathrm{P}^{(r)}$ of $\mathrm{G} \otimes_{\mathbb{Z}} \mathbb{Q}$ (when $r>0$ ), and all maximal parabolic subgroups of $G \otimes_{\mathbb{Z}} \mathbb{Q}$ are conjugate to one of these standard ones, by Lemma A.4.3. Similarly, $\mathrm{Z}_{-2}^{(r)}:=\mathrm{F}_{-2}^{(r)} \otimes_{\mathbb{Z}} \hat{\mathbb{Z}}$ is a totally isotropic $0 \otimes_{\mathbb{Z}} \hat{\mathbb{Z}}$-submodule of $L \otimes_{\mathbb{Z}} \hat{\mathbb{Z}}$, and the left $\mathrm{G}(\mathbb{Q})$ - and right $\mathscr{H}$-double orbits of $\mathrm{Z}_{-2}^{(r)}$, for $0 \leq r \leq b$, exhaust all the possible $Z_{\mathscr{H}}$ appearing in cusp labels $\left[\left(\mathrm{Z}_{\mathscr{H}}, \Phi_{\mathscr{H}}, \delta_{\mathscr{H}}\right)\right]$ for $\mathrm{M}_{\mathscr{H}}$, by Proposition A.5.8. By Lemma A.4.7, by forgetting their $p$-parts, their left $\mathrm{G}(\mathbb{Q})$ - and right $\mathscr{H}^{p}$-double orbits also exhaust all the possible $Z_{\mathscr{H}} p$ appearing in cusp labels $\left[\left(\mathrm{Z}_{\mathscr{H}^{p}}, \Phi_{\mathscr{H}^{p}}, \delta_{\mathscr{H}^{p}}\right)\right]$ for $\mathrm{M}_{\mathscr{H}^{p}}$. Let us say that a cusp label $\left[\left(\mathrm{Z}_{\mathscr{H}}, \Phi_{\mathscr{H}}, \delta_{\mathscr{H}}\right)\right]$ for $\mathrm{M}_{\mathscr{H}}$ is of rank $r$ if $\mathrm{Z}_{\mathscr{H}}$ is in the double orbit of $\mathrm{Z}_{-2}^{(r)}$, and that a cusp [ $\left.\left(\mathrm{Z}_{\mathscr{H}^{p}}, \Phi_{\mathscr{H}}, \delta_{\mathscr{H}^{p}}\right)\right]$ for M⿻丷e is of rank $r$ if it is assigned to one of rank $r$ under (A.4.1). (This is consistent with [KWL, Definitions 5.4.1.12 and 5.4.2.7].) On the other hand, as a byproduct of the proof of Proposition A.5.9, any $\mathrm{Z}_{\mathscr{H}}$ in the double orbit of $\mathrm{Z}_{-2}^{(r)}$ does extend to some cusp label $\left[\left(\mathrm{Z}_{\mathscr{H}}, \Phi_{\mathscr{H}}, \delta_{\mathscr{H}}\right)\right]$ for $\mathrm{M}_{\mathscr{H}}$, inducing some cusp label

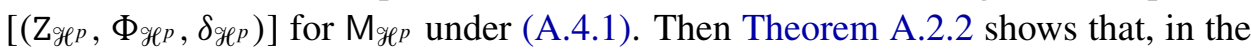
boundary stratification of every connected component of every geometric fiber of $\mathrm{M}_{\mathscr{H}}^{\min } \rightarrow \mathrm{S}_{0}=\operatorname{Spec}\left(\mathcal{O}_{F_{0},(p)}\right)$, there exist nonempty strata labeled by cusp labels for Mrgp of all possible ranks $0 \leq r \leq b$. (The theorem shows the more refined nonemptiness for strata labeled by cusp labels, not just by ranks.)

The next example shows that we cannot expect Theorem A.2.2 to be true without the requirement (in Assumption A.2.1) that $O \otimes_{\mathbb{Z}} \mathbb{Q}$ involves no factor of type D.

Example A.7.2. Suppose $\mathbb{O} \otimes_{\mathbb{Z}} \mathbb{Q}$ is a central division algebra $D$ over a totally real field $F$, as in [KWL, Proposition 1.2.1.13] such that $D \otimes_{F, \tau} \mathbb{R} \cong \mathbb{U}$, the real Hamiltonian quaternion algebra, for every embedding $\tau: F \rightarrow \mathbb{R}$, with $\star=\diamond$ given by $x \mapsto x^{\diamond}:=\operatorname{Tr}_{D / F}(x)-x$. Suppose that $D$ is nonsplit at strictly more than two places. Suppose $L$ is chosen such that $L \otimes_{\mathbb{Z}} \mathbb{Q} \cong D^{\oplus 2}$. By the Gram-Schmidt process, as in [KWL, Section 1.2.4] and by [KWL, Corollary 1.1.2.6], there is up to isomorphism only one isotropic skew-Hermitian pairing over $L \otimes_{\mathbb{Z}} \mathbb{Q}$. But we do know the failure of Hasse's principle (see [Kottwitz 1992, §7, p. 393]) in this case (see [Scharlau 1985, Remark 10.4.6]), which means there exists a choice of $(L,\langle\cdot, \cdot\rangle)$ as above that is globally anisotropic but locally isotropic everywhere. Thus, even when $k(s) \cong \mathbb{C}$, there exists some connected component $U$ of Sh $\mathscr{H}$, alg and some nonzero cusp label $\left[\left(\Phi_{\mathscr{H}}, \delta_{\mathscr{H}}\right)\right]$ for $\mathrm{M}_{\mathscr{H}}$ such that $U_{\left[\left(\Phi_{\mathscr{H}}, \delta_{\mathscr{H}}\right)\right]}=\varnothing$.

\section{Acknowledgements}

This paper is part of Wan's thesis under the supervision of Professor Christopher Skinner. The author would like to thank him for suggesting the problem and many helpful conversations. Wan thanks Kai-Wen Lan for answering many questions about the compactification of Shimura varieties, and would also like to thank the 
anonymous referees for carefully reading the paper, giving numerous suggestions on correcting some mistakes and improving the writing.

Lan was partially supported by the National Science Foundation under agreement No. DMS-1352216 and by an Alfred P. Sloan Research Fellowship.

\section{References}

[Baily and Borel 1966] W. L. Baily, Jr. and A. Borel, "Compactification of arithmetic quotients of bounded symmetric domains", Ann. of Math. (2) 84 (1966), 442-528. MR 35 \#6870 Zbl 0154.08602

[Borel 1963] A. Borel, "Some finiteness properties of adele groups over number fields", Inst. Hautes Études Sci. Publ. Math. 16 (1963), 5-30. MR 34 \#2578 Zbl 0135.08902

[Bruhat and Tits 1972] F. Bruhat and J. Tits, "Groupes réductifs sur un corps local”, Inst. Hautes Études Sci. Publ. Math. 41 (1972), 5-251. MR 48 \#6265 Zbl 0254.14017

[Casselman 1980] W. Casselman, "The unramified principal series of $\mathfrak{p}$-adic groups, I: The spherical function”, Compositio Math. 40:3 (1980), 387-406. MR 83a:22018

[Deligne and Mumford 1969] P. Deligne and D. Mumford, "The irreducibility of the space of curves of given genus”, Inst. Hautes Études Sci. Publ. Math. 36 (1969), 75-109. MR 41 \#6850 Zbl 0181.48803

[EGA III 1 1961] A. Grothendieck, "Eléments de géométrie algébrique, III: Étude cohomologique des faisceaux cohérents, I", Inst. Hautes Études Sci. Publ. Math. 11 (1961), 5-167. MR 0163910 Zbl 0118.36206

[Eischen and Wan 2014] E. Eischen and X. Wan, " $p$-adic $L$-functions of finite slope forms on unitary groups and Eisenstein series”, J. Math. Inst. Jussieu (online publication November 2014).

[Eischen et al. $\geq 2015$ ] E. Eischen, M. Harris, J. Li, and C. Skinner, " $p$-adic $L$-functions for unitary Shimura varieties, II", in preparation.

[Garrett 1984] P. B. Garrett, "Pullbacks of Eisenstein series; applications", pp. 114-137 in Automorphic forms of several variables (Katata, 1983), edited by I. Satake and Y. Morita, Progr. Math. 46, Birkhäuser, Boston, 1984. MR 86f:11039 Zbl 0544.10023

[Garrett 1989] P. B. Garrett, "Integral representations of Eisenstein series and $L$-functions", pp. 241-264 in Number theory, trace formulas and discrete groups (Oslo, 1987), edited by K. E. Aubert et al., Academic Press, Boston, 1989. MR 90e:11073 Zbl 0671.10024

[Gelbart et al. 1987] S. Gelbart, I. Piatetski-Shapiro, and S. Rallis, Explicit constructions of automorphic L-functions, Lecture Notes in Mathematics 1254, Springer, Berlin, 1987. MR 89k:11038 Zbl 0612.10022

[Harris 1984] M. Harris, "Eisenstein series on Shimura varieties", Ann. of Math. (2) 119:1 (1984), 59-94. MR 85j:11052 Zbl 0589.10030

[Hida 2004a] H. Hida, "Non-vanishing modulo $p$ of Hecke L-values", pp. 735-784 in Geometric aspects of Dwork theory, vol. I-II, edited by A. Adolphson et al., de Gruyter, Berlin, 2004. MR 2006b:11055 Zbl 1162.11342

[Hida 2004b] H. Hida, p-adic automorphic forms on Shimura varieties, Springer, New York, 2004. MR 2005e:11054 Zbl 1055.11032

[Hida 2011] H. Hida, "Irreducibility of the Igusa tower over unitary Shimura varieties", pp. 187-203 in On certain L-functions, edited by J. Arthur et al., Clay Math. Proc. 13, Amer. Math. Soc., 2011. MR 2012c:11131 Zbl 1272.11081 
[Hsieh 2011] M.-L. Hsieh, "Ordinary $p$-adic Eisenstein series and $p$-adic $L$-functions for unitary groups”, Ann. Inst. Fourier (Grenoble) 61:3 (2011), 987-1059. MR 2918724 Zbl 1271.11051

[Hsieh 2014] M.-L. Hsieh, "Eisenstein congruence on unitary groups and Iwasawa main conjectures for CM fields", J. Amer. Math. Soc. 27:3 (2014), 753-862. MR 3194494

[Ikeda 1994] T. Ikeda, "On the theory of Jacobi forms and Fourier-Jacobi coefficients of Eisenstein series”, J. Math. Kyoto Univ. 34:3 (1994), 615-636. MR 95h:11044 Zbl 0822.11041

[Kottwitz 1992] R. E. Kottwitz, "Points on some Shimura varieties over finite fields", J. Amer. Math. Soc. 5:2 (1992), 373-444. MR 93a:11053 Zbl 0796.14014

[Kudla 1994] S. S. Kudla, "Splitting metaplectic covers of dual reductive pairs", Israel J. Math. 87:1-3 (1994), 361-401. MR 95h:22019 Zbl 0840.22029

[Lan 2008] K.-W. Lan, Arithmetic compactifications of PEL-type Shimura varieties, Ph.D. thesis, Harvard University, 2008, http://search.proquest.com/docview/304599957. MR 2711676

[Lan 2011] K.-W. Lan, "Elevators for degenerations of PEL structures", Math. Res. Lett. 18:5 (2011), 889-907. MR 2875862 Zbl 1260.14026

[Lan 2012] K.-W. Lan, "Comparison between analytic and algebraic constructions of toroidal compactifications of PEL-type Shimura varieties”, J. Reine Angew. Math. 664 (2012), 163-228. MR 2980135 Zbl 1242.14022

[Lan 2013a] K.-W. Lan, Arithmetic compactifications of PEL-type Shimura varieties, London Mathematical Society Monographs Series 36, Princeton University Press, NJ, 2013. MR 3186092 Zbl 1284.14004

[Lan 2013b] K.-W. Lan, "Compactifications of PEL-Type Shimura Varieties and Kuga Families with Ordinary Loci”, preprint, 2013, http://www.math.umn.edu/ kwlan/articles/cpt-ram-ord.pdf.

[Lan 2014] K.-W. Lan, "Compactifications of PEL-type Shimura varieties in ramified characteristics", preprint, 2014, http://www.math.umn.edu/ kwlan/articles/cpt-ram-flat.pdf.

[Lapid and Rallis 2005] E. M. Lapid and S. Rallis, "On the local factors of representations of classical groups", pp. 309-359 in Automorphic representations, L-functions and applications: progress and prospects, edited by J. W. Cogdell et al., Ohio State Univ. Math. Res. Inst. Publ. 11, de Gruyter, Berlin, 2005. MR 2006j:11071 Zbl 1188.11023

[Platonov and Rapinchuk 1994] V. Platonov and A. Rapinchuk, Algebraic groups and number theory, Pure and Applied Mathematics 139, Academic Press, Boston, 1994. MR 95b:11039 Zbl 0841.20046

[Reiner 1975] I. Reiner, Maximal orders, London Mathematical Society Monographs 5, Academic Press, London-New York, 1975. MR 52 \#13910 Zbl 0305.16001

[Scharlau 1985] W. Scharlau, Quadratic and Hermitian forms, Grundlehren der Math. Wissenschaften 270, Springer, Berlin, 1985. MR 86k:11022 Zbl 0584.10010

[SGA $3_{\text {I }}$ 1970] M. Demazure and A. Grothendieck, Schémas en groupes, Tome I: Propriétés générales des schémas en groupes, Exposés I-VII (Séminaire de Géométrie Algébrique du Bois Marie 19621964), Lecture Notes in Math. 151, Springer, Berlin, 1970. MR 43 \#223a Zbl 0207.51401

[SGA 3 III 1970] M. Demazure and A. Grothendieck, Schémas en groupes, Tome III: Structure des schémas en groupes réductifs, Exposés XIX-XXVI (Séminaire de Géométrie Algébrique du Bois Marie 1962-1964), Lecture Notes in Math. 153, Springer, Berlin, 1970. MR 43 \#223c Zbl 0212.52810

[Shimura 1997] G. Shimura, Euler products and Eisenstein series, CBMS Regional Conference Series in Mathematics 93, Amer. Math. Soc., 1997. MR 98h:11057 Zbl 0906.11020

[Skinner 2014] C. Skinner, "A converse of Gross, Zagier and Kolyvagin”, preprint, 2014. arXiv 1405. 7294 
[Skinner and Urban 2014] C. Skinner and E. Urban, "The Iwasawa main conjectures for GL2", Invent. Math. 195:1 (2014), 1-277. MR 3148103 Zbl 1301.11074

[Springer 1998] T. A. Springer, Linear algebraic groups, 2nd ed., Progress in Mathematics 9, Birkhäuser, Boston, 1998. MR 99h:20075 Zbl 0927.20024

[Wan 2013] X. Wan, "Iwasawa main conjecture for Hilbert modular forms", preprint, 2013, http:// www.math.columbia.edu/ xw2295/Hilbert\%20Modular\%20Forms.pdf.

[Wan 2015] X. Wan, "The Iwasawa main conjecture for Hilbert modular forms", Forum Math. Sigma 3 (2015), e18.

Communicated by Shou-Wu Zhang

Received 2014-02-12 Revised 2015-06-27 Accepted 2015-08-18

xw2295@math.columbia.edu Department of Mathematics, Columbia University, Room 509, MC 4406, 2990 Broadway, New York, NY 10027, United States

kwlan@math.umn.edu School of Mathematics, University of Minnesota, Minneapolis, MN 55455, USA 


\section{Algebra \& Number Theory}

msp.org/ant

\section{EDITORS}

MANAGING EDITOR

Bjorn Poonen

Massachusetts Institute of Technology

Cambridge, USA

\author{
EDITORIAL BOARD CHAIR \\ David Eisenbud \\ University of California \\ Berkeley, USA
}

BOARD OF EDITORS

Georgia Benkart

Dave Benson

Richard E. Borcherds

John H. Coates

J-L. Colliot-Thélène

Brian D. Conrad

Hélène Esnault

Hubert Flenner

Sergey Fomin

Edward Frenkel

Andrew Granville

Joseph Gubeladze

Roger Heath-Brown

Craig Huneke

Kiran S. Kedlaya

János Kollár

Yuri Manin

Philippe Michel
University of Wisconsin, Madison, USA

University of Aberdeen, Scotland

University of California, Berkeley, USA

University of Cambridge, UK

CNRS, Université Paris-Sud, France

Stanford University, USA

Freie Universität Berlin, Germany

Ruhr-Universität, Germany

University of Michigan, USA

University of California, Berkeley, USA

Université de Montréal, Canada

San Francisco State University, USA

Oxford University, UK

University of Virginia, USA

Univ. of California, San Diego, USA

Princeton University, USA

Northwestern University, USA

École Polytechnique Fédérale de Lausanne
Susan Montgomery

Shigefumi Mori

Raman Parimala

Jonathan Pila

Anand Pillay

Victor Reiner

Peter Sarnak

Joseph H. Silverman

Michael Singer

Vasudevan Srinivas

J. Toby Stafford

Ravi Vakil

Michel van den Bergh

Marie-France Vignéras

Kei-Ichi Watanabe

Efim Zelmanov

Shou-Wu Zhang
University of Southern California, USA

RIMS, Kyoto University, Japan

Emory University, USA

University of Oxford, UK

University of Notre Dame, USA

University of Minnesota, USA

Princeton University, USA

Brown University, USA

North Carolina State University, USA

Tata Inst. of Fund. Research, India

University of Michigan, USA

Stanford University, USA

Hasselt University, Belgium

Université Paris VII, France

Nihon University, Japan

University of California, San Diego, USA

Princeton University, USA

\section{PRODUCTION}

production@msp.org

Silvio Levy, Scientific Editor

See inside back cover or msp.org/ant for submission instructions.

The subscription price for 2015 is US $\$ 255 /$ year for the electronic version, and $\$ 440 /$ year ( $+\$ 55$, if shipping outside the US) for print and electronic. Subscriptions, requests for back issues and changes of subscribers address should be sent to MSP.

Algebra \& Number Theory (ISSN 1944-7833 electronic, 1937-0652 printed) at Mathematical Sciences Publishers, 798 Evans Hall \#3840, c/o University of California, Berkeley, CA 94720-3840 is published continuously online. Periodical rate postage paid at Berkeley, CA 94704, and additional mailing offices.

ANT peer review and production are managed by EditFLOW ${ }^{\circledR}$ from MSP.

\section{PUBLISHED BY}

- mathematical sciences publishers

nonprofit scientific publishing

http://msp.org/

(C) 2015 Mathematical Sciences Publishers 


\section{Algebra \& Number Theory}

Volume $9 \quad$ No. $9 \quad 2015$

Families of nearly ordinary Eisenstein series on unitary groups XIN WAN

Classifying orders in the Sklyanin algebra

Daniel Rogalski, Susan J. SierRa and J. Toby STAFFord

Chongying Dong, XingJun Lin and SiU-Hung NG

KAISA MATOMÄKI, MAKSYM RADZIWIŁŁ and TERENCE TAO 